Portland State University

PDXScholar

Summer 8-18-2019

\title{
Modeling Vertical Migration of Cyanobacteria and Zooplankton
}

Corina Christina Mae Overman

Portland State University

Follow this and additional works at: https://pdxscholar.library.pdx.edu/open_access_etds

Part of the Civil and Environmental Engineering Commons Let us know how access to this document benefits you.

Recommended Citation

Overman, Corina Christina Mae, "Modeling Vertical Migration of Cyanobacteria and Zooplankton" (2019). Dissertations and Theses. Paper 5178.

https://doi.org/10.15760/etd.7054

This Thesis is brought to you for free and open access. It has been accepted for inclusion in Dissertations and Theses by an authorized administrator of PDXScholar. Please contact us if we can make this document more accessible: pdxscholar@pdx.edu. 
Modeling Vertical Migration of Cyanobacteria and Zooplankton

by

Corina Christina Mae Overman

A thesis submitted in partial fulfillment of the requirements for the degree of

Master of Science

in

Civil and Environmental Engineering

\author{
Thesis Committee: \\ Scott Wells, Chair \\ Chris Berger \\ Angela Strecker
}

Portland State University

2019 
(C) 2019 Corina Christina Mae Overman 


\begin{abstract}
Cyanobacteria, also known as blue-green algae, often cause harmful algal blooms and release toxic substances that can harm humans and other animals. Accurately modeling these phytoplankton is a step towards predicting, preventing, and controlling such blooms. Cyanobacteria and zooplankton species are known to migrate vertically in the water column on a daily cycle. Capturing this behavior is one aspect of correctly modeling their dynamics. Here, several models of cyanobacteria vertical movement were tested in proof-of-concept models before being applied to data from field studies. These models included both continuum and particle-tracking frameworks. Four continuum-framework models of cyanobacteria vertical migration were chosen to add to the numerical hydrodynamic, waterquality model CE-QUAL-W2. These were tested using a model of Dexter Reservoir (Oregon), where they predicted vertical migratory movement seen in cyanobacteria. Models of zooplankton migration were also tested in proof-of-concept models as a steppingstone towards future incorporation into CE-QUAL-W2. Preliminary models of cyanobacteria and zooplankton vertical migration using a particle-tracking framework also provided information to be used in future model developments that will incorporate vertical migration of plankton into the particle-tracking module of CE-QUAL-W2.
\end{abstract}




\section{Acknowledgements}

I would like to thank my thesis committee chair, Dr. Scott Wells, for his enthusiastic support and commitment to teaching. I would also like to thank my committee members, Dr. Chris Berger and Dr. Angela Strecker, for their time and expertise. Additionally, I would like to thank my fellow graduate students in the Water Quality Modeling Group, Amory Cervarich and tel jensen, for generously offering suggestions, assistance, and encouragement. Financial support was provided by a Selected Professions Fellowship from the American Association of University Women, a Maseeh Fellowship from the Maseeh College of Engineering and Computer Science, and funding from the United States Army Corps of Engineers in modeling Dexter Reservoir. 


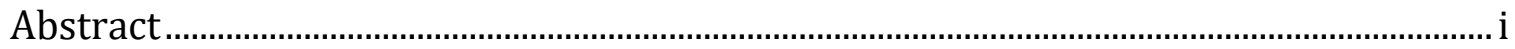

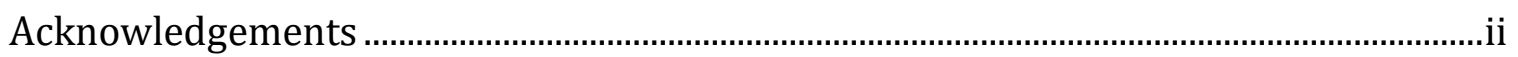

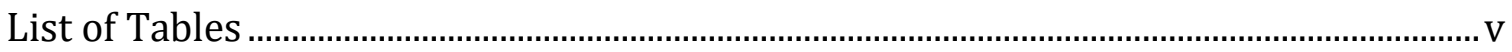

List of Figures

Chapter 1: Introduction ..................................................................................................

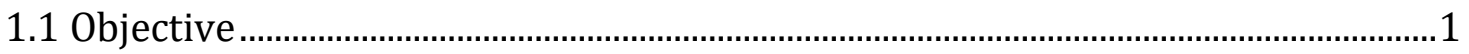

1.2 Review of Plankton Vertical Migration Modeling ......................................................

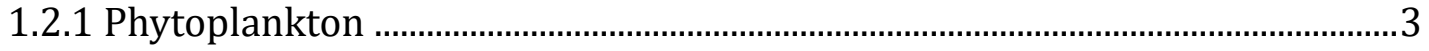

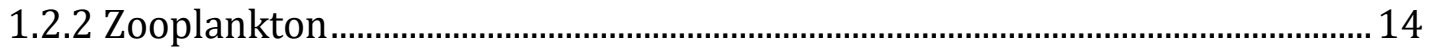

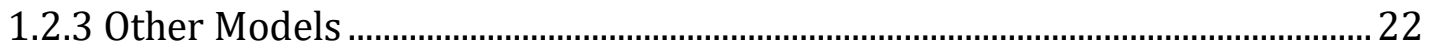

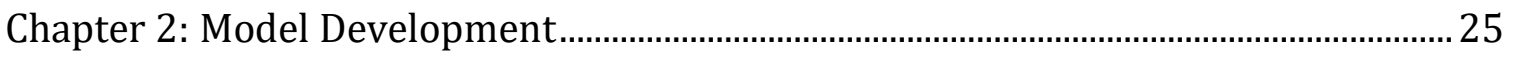

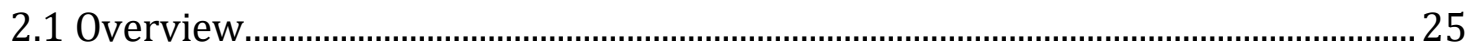

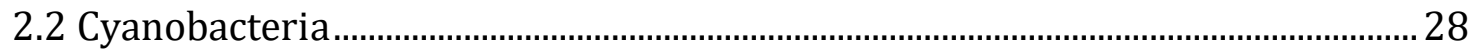

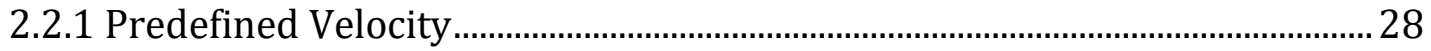

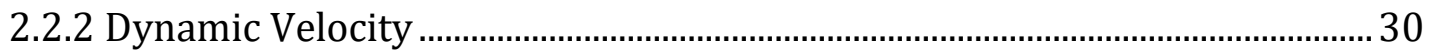

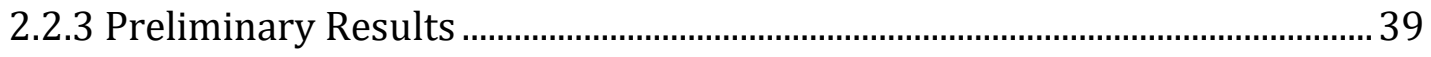

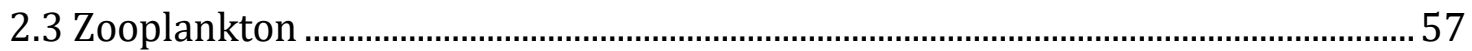

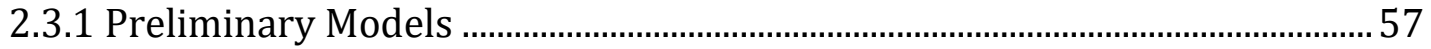

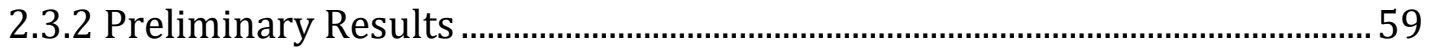

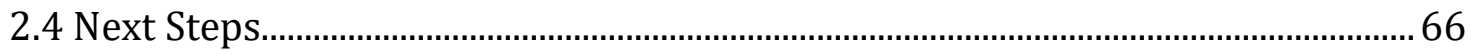

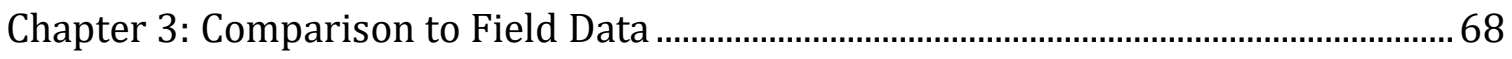

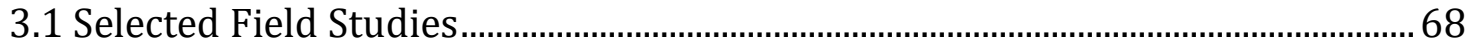

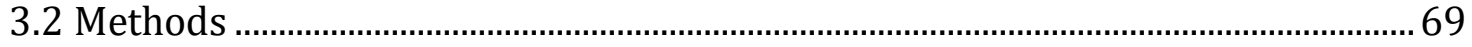

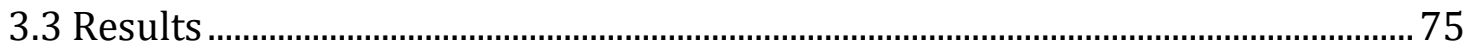

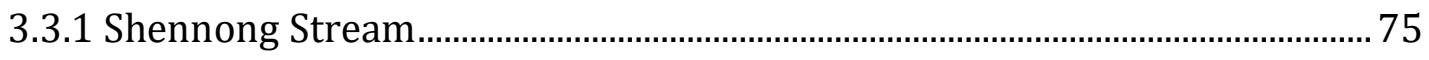

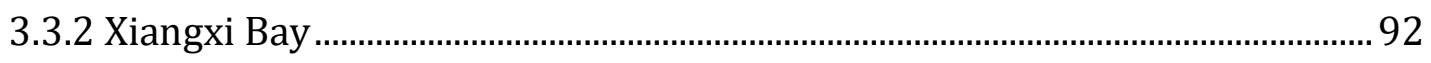

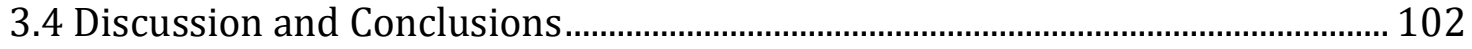

Chapter 4: Integration into CE-QUAL-W2 ….................................................................. 105 


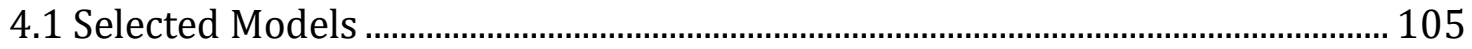

4.2 Test Case: Dexter Reservoir ...................................................................................... 110

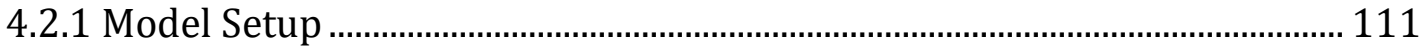

4.2.2 Results....................................................................................................... 115

4.2.3 Discussion and Conclusions .............................................................................. 131

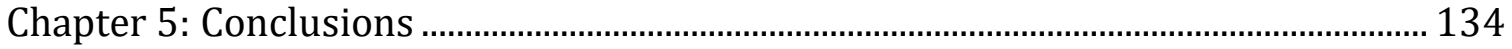

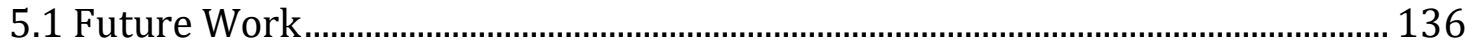

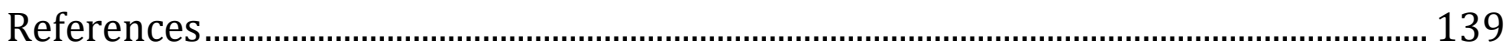

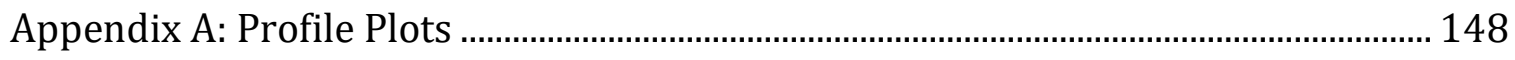

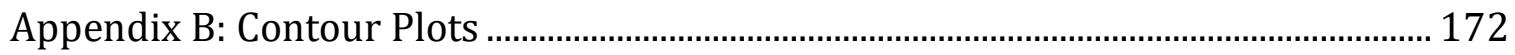

Appendix C: CE-QUAL-W2 Code....................................................................................... 185 


\section{List of Tables}

Table 1-1 Freshwater cyanobacteria genera, toxins, and health effects, extracted from Lopez et al., 2008 (Paerl et al., 2001; Fristachi et al., 2008; HARRNESS, 2005;

Falconer, 2005).

Table 1-2. Equations used in studies and models of cyanobacteria vertical migration

Table 1-3. Equations used in studies and models of zooplankton light-dependent

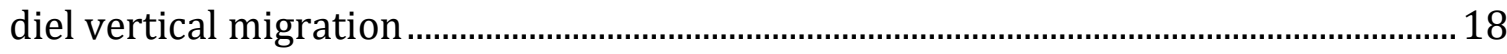

Table 2-1, Predefined velocity models used in continuum framework ......................... 29

Table 2-2 Predefined velocity models used in particle-tracking framework .............. 29

Table 2-3. Dynamic velocity models used in continuum framework................................ 35

Table 2-4. Dynamic velocity models used in particle-tracking framework.................... 36

Table 2-5 Converted parameter values from Visser et al. (1997)..................................... 37

Table 2-6 Literature values for biological parameters of cyanobacteria used in

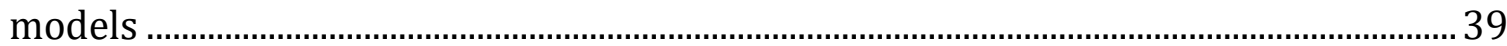

Table 2-7 Values used in setup of preliminary models........................................................ 41

Table 2-8 Values used in preliminary predefined velocity models .................................. 42

Table 2-9 Values used in preliminary growth kinetics models ......................................... 46

Table 2-10 Values used in preliminary models based on Visser et al. (1997).............50

Table 2-11 Values used in preliminary light function models .......................................... 54

Table 2-12 Values used in preliminary models of zooplankton migration ..................59

Table 3-1 Ranges of values used in model applications to field study data .................. 70

Table 3-2 Error statistics from models of Shennong Stream enclosure site

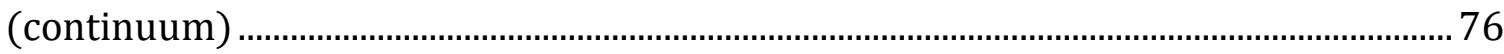

Table 3-3 Error statistics from models of Shennong Stream enclosure site (particletracking) 81

Table 3-4 Error statistics from models of Shennong Stream open water site (continuum).

Table 3-5 Error statistics from models of Shennong Stream open water site (particle-tracking)

Table 3-6 Error statistics from models of Xiangxi Bay with $D_{z}=10^{-5} \mathrm{~m}^{2} \mathrm{~s}^{-1}$ (continuum) 
Table 3-7 Error statistics from models of Xiangxi Bay with $D_{z}=10^{-4} \mathrm{~m}^{2} \mathrm{~s}^{-1}$ (continuum)

Table 3-8 Error statistics from models of Xiangxi Bay with $D_{z}=10^{-5} \mathrm{~m}^{2} \mathrm{~s}^{-1}$ (particletracking)

Table 3-9 Error statistics from models of Xiangxi Bay with $D_{z}=10^{-4} \mathrm{~m}^{2} \mathrm{~s}^{-1}$ (particletracking)

Table 4-1 User-defined variables for migration Models $1 \& 2$ (time-varying velocity and Belov \& Giles [1997])

Table 4-2 User-defined variables for migration Model 3 (light function with time decay)

Table 4-3 User-defined variables for migration Model 4 (Visser et al. [1997]) ....... 110

Table 4-4 Variables used in application of CE-QUAL-W2 algae migration Models 1 \& 2 to Dexter Reservoir model.

Table 4-5 Variables used in application of CE-QUAL-W2 algae migration Model 3 to Dexter Reservoir model.

Table 4-6 Variables used in application of CE-QUAL-W2 algae migration Model 4 to Dexter Reservoir model.

Table 4-7 Error statistics comparing output from model segment 10 to measured chlorophyll a data, east basin sampling site.

Table 4-8 Error statistics comparing output from model segment 21 to measured chlorophyll $a$, west basin sampling site

Table 4-9 Error statistics comparing model output to measured chlorophyll $a$, both sampling sites

Table 4-10 Error statistics comparing model output to dissolved oxygen (D0) data

Table 4-11 Error statistics comparing model output to temperature data. 


\section{List of Figures}

Figure 2-1 Layout of model grid used in preliminary models 26

Figure 2-2 Five-day time series of concentration at depth predicted by preliminary

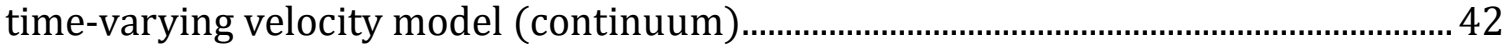

Figure 2-3 Five-day time series of concentration at depth predicted by preliminary time-varying velocity model (particle-tracking)

Figure 2-4 Concentration profiles at two-hour intervals predicted by preliminary time-varying velocity models in continuum and particle-tracking frameworks ........ 43

Figure 2-5 Five-day time series of concentration at depth predicted by preliminary Belov \& Giles (1997) model (continuum). 44

Figure 2-6 Five-day time series of concentration at depth predicted by preliminary Belov \& Giles (1997) model (particle-tracking). 44

Figure 2-7 Concentration profiles at two-hour intervals predicted by preliminary Belov \& Giles (1997) models in continuum and particle-tracking frameworks ......... 45 Figure 2-8 Five-day time series of concentration at depth predicted by preliminary growth kinetics model without time decay (continuum).

Figure 2-9 Five-day time series of concentration at depth predicted by preliminary growth kinetics model with time decay (continuum) 47

Figure 2-10 Five-day time series of concentration at depth predicted by preliminary growth kinetics model (particle-tracking).

Figure 2-11 Concentration profiles at two-hour intervals predicted by preliminary growth kinetics models (with and without time decay) in continuum and particletracking frameworks

Figure 2-12 Five-day time series of concentration at depth predicted by preliminary Visser et al. (!997) model (continuum) 51

Figure 2-13 Five-day time series of concentration at depth predicted by preliminary Visser et al. (!997) model (particle-tracking) 52

Figure 2-14 Concentration profiles at two-hour intervals predicted by preliminary Visser et al. (1997) models in continuum and particle-tracking frameworks. 53

Figure 2-15 Five-day time series of concentration at depth predicted by preliminary light function model without time decay (continuum) 54

Figure 2-16 Five-day time series of concentration at depth predicted by preliminary light function model with time decay (continuum) 55 
Figure 2-17 Five-day time series of concentration at depth predicted by preliminary light function model (particle-tracking) ............................................................................5

Figure 2-18 Concentration profiles at two-hour intervals predicted by preliminary light function models (with and without time decay) in continuum and particletracking frameworks 57

Figure 2-19 Five-day time series of concentration at depth predicted by preliminary Dodson (1990) model (continuum). 60

Figure 2-20 Five-day time series of concentration at depth predicted by preliminary

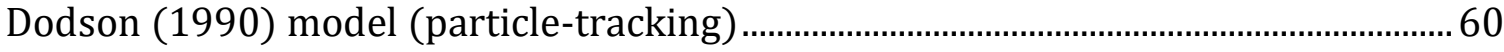

Figure 2-21 Concentration profiles at two-hour intervals predicted by preliminary Dodson (1990) models in continuum and particle-tracking frameworks. 61

Figure 2-22 Five-day time series of concentration at depth predicted by preliminary Andersen \& Nival (1991) model (continuum). 62

Figure 2-23 Five-day time series of concentration at depth predicted by preliminary Andersen \& Nival (1991) model (particle-tracking)..

Figure 2-24 Concentration profiles at two-hour intervals predicted by preliminary Andersen \& Nival (1991) models in continuum and particle-tracking frameworks 64

Figure 2-25 Five-day time series of concentration at depth predicted by preliminary

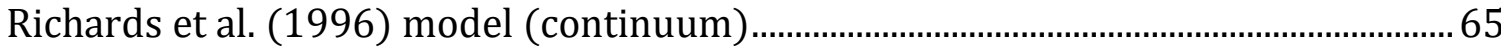

Figure 2-26 Five-day time series of concentration at depth predicted by preliminary Richards et al. (1996) model (particle-tracking).

Figure 2-27 Concentration profiles at two-hour intervals predicted by preliminary Richards et al. (1996) models in continuum and particle-tracking frameworks .......66

Figure 3-1 Chlorophyll a concentration profiles measured in Shennong stream enclosure (dark dots) and open water (light dots) sites, extracted from Cui et al. (2016)

Figure 3-2 Morisita's Index ( $\mathrm{I}_{\mathrm{s}}$, bars) and mean residence depth (MRD, dots) calculated from chlorophyll a profiles measured in Shennong Stream open water and enclosure sites, extracted from Cui et al. (2016)

Figure 3-3 Time series of chlorophyll a contours measured at Xiangxi Bay with depth of maximum chlorophyll a concentration shown by dashed line, extracted from Wang et al. (2011)..

Figure 3-4 Mean residence depth (MRD, bars) and Morisita's Index (MI, dotted line) calculated from chlorophyll $a$ data from Xiangxi Bay, extracted from Wang et al. (2011) 
Figure 3-5 Time series of observed and predicted mean residence depth of chlorophyll a concentration in Shennong Stream enclosure site using predefined velocity models (continuum)

Figure 3-6 Time series of observed and predicted mean residence depth of chlorophyll a concentration in Shennong Stream enclosure site using dynamic velocity models (continuum)

Figure 3-7 Time series of observed and predicted chlorophyll a concentration in Shennong Stream enclosure site using predefined velocity models (continuum) ..... 79

Figure 3-8 Time series of observed and predicted chlorophyll a concentration in Shennong Stream enclosure site using dynamic velocity models (continuum).......... 80

Figure 3-9 Time series of observed and predicted mean residence depth of chlorophyll a concentration in Shennong Stream enclosure site using predefined velocity models (particle-tracking)

Figure 3-10 Time series of observed and predicted mean residence depth of chlorophyll a concentration in Shennong Stream enclosure site using dynamic velocity models (particle-tracking)

Figure 3-11 Time series of observed and predicted chlorophyll a concentration in Shennong Stream enclosure site using predefined velocity models (particletracking)

Figure 3-12 Time series of observed and predicted chlorophyll a concentration in Shennong Stream enclosure site using dynamic velocity models (particle-tracking)

Figure 3-13 Time series of observed and predicted mean residence depth of chlorophyll a concentration in Shennong Stream open water site using predefined velocity models (continuum)

Figure 3-14 Time series of observed and predicted mean residence depth of chlorophyll a concentration in Shennong Stream open water site using dynamic velocity models (continuum)

Figure 3-15 Time series of observed and predicted chlorophyll a concentration in Shennong Stream open water site using predefined velocity models (continuum) . 87

Figure 3-16 Time series of observed and predicted chlorophyll a concentration in Shennong Stream open water site using dynamic velocity models (continuum) ...... 88

Figure 3-17 Time series of observed and predicted mean residence depth of chlorophyll a concentration in Shennong Stream open water site using predefined velocity models (particle-tracking) 
Figure 3-18 Time series of observed and predicted mean residence depth of chlorophyll a concentration in Shennong Stream open water site using dynamic velocity models (particle-tracking)

Figure 3-19 Time series of observed and predicted chlorophyll a concentration in Shennong Stream open water site using predefined velocity models (particletracking)

Figure 3-20 Time series of observed and predicted chlorophyll a concentration in Shennong Stream open water site using dynamic velocity models (particle-tracking)

Figure 3-21 Time series of observed and predicted mean residence depth of chlorophyll a concentration in Xiangxi Bay using predefined velocity models (continuum)

Figure 3-22 Time series of observed and predicted mean residence depth of chlorophyll a concentration in Xiangxi Bay using dynamic velocity models (continuum)

Figure 3-23 Time series of observed and predicted depth of maximum chlorophyll $a$ concentration in Xiangxi Bay using predefined velocity models (continuum)............ 96

Figure 3-24 Time series of observed and predicted depth of maximum chlorophyll $a$ concentration in Xiangxi Bay using dynamic velocity models (continuum). 97

Figure 3-25 Time series of observed and predicted mean residence depth of chlorophyll a concentration in Xiangxi Bay using predefined velocity models (particle-tracking) 100

Figure 3-26 Time series of observed and predicted mean residence depth of chlorophyll a concentration in Xiangxi Bay using dynamic velocity models (particletracking)

Figure 3-27 Time series of observed and predicted depth of maximum chlorophyll $a$ concentration in Xiangxi Bay using predefined velocity models (particle-tracking)

Figure 3-28 Time series of observed and predicted depth of maximum chlorophyll a concentration in Xiangxi Bay using dynamic velocity models (particle-tracking) . 101

Figure 4-1 Map of Dexter Reservoir showing data collection sites, extracted from Berger et al. (2018)

Figure 4-2 Predicted concentration of Algal Group 2 in segment 10 (east basin sampling site) with constant settling velocity.

Figure 4-3 Predicted concentration of Algal Group 2 in segment 21 (west basin sampling site) with constant settling velocity. 
Figure 4-4 Observed and predicted chlorophyll a profiles at east basin (segment 10, left) and west basin (segment 21, right) sampling sites using constant settling velocity, 28 Jun. 2016

Figure 4-5 Observed and predicted chlorophyll a profiles at east basin (segment 10, left) and west basin (segment 21, right) sampling sites using constant settling velocity, 8 Aug. 2016.

Figure 4-6 Observed and predicted chlorophyll a profiles at east basin (left) and west basin (right) sampling sites using constant settling velocity, 19 Oct. 2016.... 118

Figure 4-7 Predicted concentration of Algal Group 2 in segment 10 (east basin sampling site) using Model 1 (time-varying velocity).

Figure 4-8 Predicted concentration of Algal Group 2 in segment 21 (west basin sampling site) using Model 1 (time-varying velocity)

Figure 4-9 Predicted concentration of Algal Group 2 in segment 10 (east basin sampling site) using Model 2 (Belov \& Giles [1997])

Figure 4-10 Predicted concentration of Algal Group 2 in segment 21 (west basin sampling site) using Model 2 (Belov \& Giles [1997])

Figure 4-11 Predicted concentration of Algal Group 2 in segment 10 (east basin sampling site) using Model 3 (light function with time decay).

Figure 4-12 Predicted concentration of Algal Group 2 in segment 21 (west basin sampling site) using Model 3 (light function with time decay).

Figure 4-13 Predicted concentration of Algal Group 2 in segment 10 (east basin sampling site) using Model 4 (Visser et al. [1997])

Figure 4-14 Predicted concentration of Algal Group 2 in segment 21 (west basin sampling site) using Model 4 (Visser et al. [1997])

Figure 4-15 Observed and predicted chlorophyll a profiles at east basin (segment 10, left) and west basin (segment 21, right) sampling sites using Model 3 (light function with time decay), 28 Jun. 2016.

Figure 4-16 Observed and predicted chlorophyll $a$ profiles at east basin (segment 10, left) and west basin (segment 21, right) sampling sites using Model 3 (light function with time decay), 8 Aug. 2016

Figure 4-17 Observed and predicted chlorophyll a profiles at east basin (segment 10, left) and west basin (segment 21, right) sampling sites using Model 3 (light function with time decay), 19 Oct. 2016.

Figure 4-18 Profile view of predicted temperature (upper frame) and concentration of Algal Group 2 (lower frame) in Dexter reservoir using Model 3 (light function with time decay), 12:00am, 29 July 2017. 
Figure 4-19 Profile view of predicted temperature (upper frame) and Algal Group 2 concentration (lower frame) in Dexter reservoir using Model 3 (light function with time decay), 6:00am, 29 July 2017................................................................................. 129

Figure 4-20 Profile view of predicted temperature (upper frame) and concentration of Algal Group 2 (lower frame) in Dexter reservoir using Model 3 (light function with time decay), 12:00pm, 29 July 2017 130

Figure 4-21 Profile view of predicted temperature (upper frame) and concentration of Algal Group 2 (lower frame) in Dexter reservoir using Model 3 (light function with time decay), 6:00pm, 29 July 2017 130

Figure 4-22 Profile view of predicted temperature (upper frame) and concentration of Algal Group 2 (lower frame) in Dexter reservoir using Model 3 (light function with time decay), 12:00am, 30 July 2017. 131 


\section{Chapter 1: Introduction}

\subsection{Objective}

The purpose of this study is to develop models of the independent vertical motility of cyanobacteria and zooplankton and to incorporate those models into CEQUAL-W2, a laterally-averaged, two-dimensional, coupled water-quality and hydrodynamic model managed by Portland State University (Cole and Wells, 2018).

Cyanobacteria are often responsible for harmful algae blooms (HABs), which occur when these phytoplankton grow excessively in a waterbody. Certain species of cyanobacteria can produce substances toxic to humans and animals (Table 1-1). Some are also able to move vertically in the water column by their own motility, independent of water velocity (Visser et al., 2016),

Zooplankton perform similar vertical movements in the water column, often cycling over a period of one day (Cohen and Forward, 2009). Recently, some studies have focused on the question of whether the mass vertical migration of marine species of zooplankton can have an effect on turbulence and mixing in the ocean (e.g. Dewar et al., 2006; Kunze et al., 2006; Katija and Dabiri, 2009; Wilhelmus and Dabiri, 2014). Fewer studies have been done on the possible effect freshwater zooplankton migration may have in lakes, making it another area of active research (Simoncelli et al., 2017). 
Table 1-1 Freshwater cyanobacteria genera, toxins, and health effects, extracted from Lopez et al., 2008 (Paerl et al., 2001; Fristachi et al., 2008; HARRNESS, 2005; Falconer, 2005)

\begin{tabular}{|c|c|c|c|}
\hline Toxin & Genera & $\begin{array}{c}\text { Short Term Health } \\
\text { Effects }\end{array}$ & $\begin{array}{c}\text { Long Term } \\
\text { Health Effects }\end{array}$ \\
\hline Microcystins & $\begin{array}{l}\text { Anabaena, } \\
\text { Aphanocapsa, } \\
\text { Hapalosphon, } \\
\text { Microcystis, Nostoc, } \\
\text { Oscillatoria, } \\
\text { Planktothrix } \\
\end{array}$ & $\begin{array}{l}\text { Gastrointestinal, liver } \\
\text { inflammation, and } \\
\text { hemorrhage and liver } \\
\text { failure leading to death, } \\
\text { pneumonia, dermatitis }\end{array}$ & $\begin{array}{l}\text { Tumor promoter, } \\
\text { liver failure } \\
\text { leading to death }\end{array}$ \\
\hline Nodularins & Nodularia spumigena & Similar to Microcystins & $\begin{array}{l}\text { Similar to } \\
\text { Microcystins }\end{array}$ \\
\hline Saxitoxins & $\begin{array}{l}\text { Anabaena, } \\
\text { Aphanizomenon, } \\
\text { Cylindrospermopsis, } \\
\text { Lyngbya }\end{array}$ & $\begin{array}{l}\text { Tingling, burning, } \\
\text { numbness, drowsiness, } \\
\text { incoherent speech, } \\
\text { respiratory paralysis } \\
\text { leading to death }\end{array}$ & Unknown \\
\hline Anatoxins & $\begin{array}{l}\text { Anabaena, } \\
\text { Aphanizomenon, } \\
\text { Oscillatoria, } \\
\text { Planktothrix }\end{array}$ & $\begin{array}{l}\text { Tingling, burning, } \\
\text { numbness, drowsiness, } \\
\text { incoherent speech, } \\
\text { respiratory paralysis } \\
\text { leading to death }\end{array}$ & $\begin{array}{l}\text { Cardiac } \\
\text { arrhythmia } \\
\text { leading to death }\end{array}$ \\
\hline Cylindrospermopsin & $\begin{array}{l}\text { Aphanizomenon, } \\
\text { Cylindrospermopsis, } \\
\text { Umezakia }\end{array}$ & $\begin{array}{l}\text { Gastrointestinal, liver } \\
\text { inflammation and } \\
\text { hemorrhage, pneumonia, } \\
\text { dermatitis }\end{array}$ & $\begin{array}{l}\text { Malaise, anorexia, } \\
\text { liver failure } \\
\text { leading to death }\end{array}$ \\
\hline Lipopolysaccharide & $\begin{array}{l}\text { Aphanizomenon, } \\
\text { Oscillatoria }\end{array}$ & Gastrointestinal, dermatitis & Unknown \\
\hline Lyngbyatoxins & Lyngbya & Dermatitis & $\begin{array}{l}\text { Skin tumors } \\
\text { (Fujiki et al. } \\
\text { 1990), unknown }\end{array}$ \\
\hline BMAA & $\begin{array}{l}\text { Anabaena, } \\
\text { Cylindrospermopsin, } \\
\text { Microcystis, Nostoc, } \\
\text { Planktothrix } \\
\end{array}$ & & $\begin{array}{l}\text { Potential link to } \\
\text { neurodegenerative } \\
\text { diseases }\end{array}$ \\
\hline
\end{tabular}

Due to the serious health effects caused by HABs and their increasing frequency of occurrence in waterbodies (Hudnell, 2010), it is desirable to be able to model the organisms responsible for them more accurately. Similarly, zooplankton should be modeled realistically since they are an important part of aquatic ecosystems. In the current version of CE-QUAL-W2, all phyto- and zooplankton are modeled as a continuum concentration (i.e., well-mixed in a model computational 
cell or control volume) with a constant floating or sinking velocity. This work aims to develop models of cyanobacteria and zooplankton vertical motility that can be incorporated into CE-QUAL-W2. This includes models based on the existing continuum-concentration approach used for plankton as well as a particle-tracking approach. CE-QUAL-W2 has particle-tracking capabilities, but these have yet to be applied to plankton.

\subsection{Review of Plankton Vertical Migration Modeling}

\subsubsection{Phytoplankton}

Phytoplankton are planktonic organisms that make their own food through photosynthesis. One of the most-studied types of phytoplankton is cyanobacteria. Although these are taxonomically classified as bacteria, they are often referred to as "blue-green algae." Several genera of cyanobacteria, including Microcystis, Oscillatoria, Anabaena, and Aphanizomenon, produce toxic substances called cyanotoxins, which can cause serious health problems in humans and other mammals (Howard, 1994). Some of these species also migrate vertically in the water column, enabling them to rise to the water surface where growing conditions are favorable. The vertical migration of species such as Microcystis aeruginosa, Oscillatoria agardhii, and Anabaena flos-aqua can lead to HABs when colonies accumulate on a water surface and experience increased growth, causing degradation of water quality and environmental health (Belov and Giles, 1997). 
Vertical migration is thought to be beneficial to cyanobacteria because it may allow them to travel between the surface layers of a waterbody, where light is abundant, and lower, more nutrient-rich layers (Brookes and Ganf, 2001). Some studies suggest that cyanobacteria are able to move past the thermocline of a lake to take advantage of nutrients in the hypolimnion (Ganf and Oliver, 1982), while others found insufficient evidence that this occurs in natural systems (Bormans et al., 1999). Nevertheless, this movement is achieved through a process called buoyancy regulation. Cells regulate their buoyancy either with carbohydrate ballast or gas vesicles (Kromkamp and Walsby, 1990). Carbohydrates are accumulated when cells photosynthesize, and this ballast causes a decrease in buoyancy and subsequent sinking. Once cells have stopped photosynthesizing, the carbohydrates are consumed, the ballast depleted, and cells rise again (Kromkamp and Mur, 1984). Chemicals accumulated during photosynthesis also cause gas vesicles contained in cells to collapse through turgor pressure, which decreases buoyancy. Synthesis of new gas vesicles leads to an increase in buoyancy (Kromkamp et al., 1988).

Based on these mechanisms, buoyancy regulation and vertical migration are affected by external factors such as light and nutrients. Laboratory experiments on O. agardhii showed that carbohydrate ballast (and, therefore, density) increased with increasing irradiance, then leveled off and eventually decreased after light ceased (Kromkamp and Walsby, 1990). Ibelings et al. (1991) observed that Microcystis colonies in two lakes in the Netherlands decreased in buoyancy during 
the day and increased at night, following a diurnal light cycle. Similar results were found by Cui et al. (2016) in the Three Gorges Reservoir. Visser et al. (1997) found a positive relationship between carbohydrate content and density of Microcystis cells in laboratory experiments. They also found that the rate of cell density change increased with increasing photon irradiance up to a point, then decreased as photon irradiance continued to increase. Their experiments showed that after light ceased, the rate of density decrease was greater when initial cell density was greater. Wallace and Hamilton (1999) performed similar experiments on M. aeruginosa in the laboratory and confirmed the positive relationship between cell density and carbohydrate content found by Visser et al. (1997). They also proposed the existence of a "response time" that occurs when cells are first exposed to light. Until the end of the response time, cell density does not increase constantly with light. Laboratory experiments on M. aeruginosa suggest that buoyancy regulation is dependent on the light and nutrient history experienced by cells, as well as persisting light and nutrient conditions (Brookes and Ganf, 2001).

Exogenous factors besides light and nutrients have been found to affect cyanobacteria distributions. In Lake Taihu in China, surface blooms of M. aeruginosa did not form when wind speed and surface wave height exceeded critical values of $3.1 \mathrm{~m} / \mathrm{s}$ and $0.062 \mathrm{~m}$, respectively (Cao et al., 2006). Microcystis colonies in the Three Gorges Reservoir were observed to migrate to greater depths in open water while those in a protected enclosure stayed closer to the surface (Cui et al., 2016). 
Zhao et al. (2017) found that Microcystis spp. (mainly M. aeruginosa) in a laboratory experiment could maintain buoyancy up to a critical value of turbulent kinetic energy, and that this value increased with colony size. They hypothesized that larger colonies were more able to overcome turbulent entrainment due to their greater diameter, which increases drag force. Similar results were found by Zhu et al. (2018) in Lake Taihu. In response to the tendency of cyanobacteria species to thrive in stratified systems, artificial mixing techniques are often used to control and prevent blooms. These include aeration and pumping water between the hypolimnion and epilimnion to decrease stratification and increase turbulence (Visser et al., 2016). This disrupts the stability that allows the cyanobacteria to stay at the water surface and can displace them to deeper parts of the water column where growing conditions are less favorable.

Because of the threat cyanobacteria poses to environmental and human health and the observed relationship between cyanobacteria movement and measurable variables, many modeling efforts have focused on predicting vertical migration. The first mechanistic computer model of cyanobacteria vertical migration was based only on the influence of turgor pressure as a function of light (Okada and Aiba, 1983a, 1983b). Kromkamp and Walsby (1990) found this model to be over-simplified in its neglect of carbohydrate ballast as a factor in buoyancy regulation. They created a model based on relationships found in laboratory 
experiments on $O$. agardhii that predicts cell density as a function of irradiance at depth.

In their model, the rate of change in density with irradiance is given by Equation 1,

$$
\frac{d \rho}{d t}=c_{1}\left(\frac{I}{K_{I}+I}\right)-c_{2} I_{a}-c_{3}
$$

where $\rho$ is the density of a cyanobacteria colony, $t$ is time, $I$ is irradiance at the depth of the colony, $K_{I}$ is the half-saturation irradiance, and $c_{1}, c_{2}$, and $c_{3}$ are rate coefficients determined from data. The previous irradiance $\left(I_{a}\right)$ is the average irradiance experienced by the colony since the start of the most recent photoperiod. When the colony does not receive any light, Equation 1 reduces to the second and third terms and predicts that density decreases. The predicted density of a cell is used to find its settling velocity $(v)$ following Stokes' law (Equation 2),

$$
v=\frac{2 g r^{2}\left(\rho_{c}-\rho^{\prime}\right) A}{9 \phi n}
$$

where $g$ is acceleration due to gravity, $r$ is colony radius, $\rho_{c}$ is cyanobacteria density, $\rho^{\prime}$ is density of water, $A$ is the ratio of cell volume to colony volume, $\phi$ is form resistance, and $n$ is viscosity of water. This velocity is used to calculate the new position after a timestep (Equation 3), 


$$
z_{2}=v P+z_{1}
$$

where $z_{2}$ and $z_{1}$ are the new and old depths, respectively, and $P$ is the time interval. This general structure, with modifications, has been used for later models.

Howard et al. (1996) built upon the model of Kromkamp and Walsby (1990), which they asserted made it more appropriate for Microcystis. This included adding algorithms for allocating carbon acquired through photosynthesis to growth, ballast, and maintenance. Cyanobacteria photosynthetic rate $\left(P_{q i}\right)$ as a function of light at depth is based on a photosynthesis/irradiance curve. Increase or decrease in cell carbohydrate ballast is based on the following algorithm:

$$
\begin{gathered}
\text { If } P_{q i}-R \leq C_{\text {gmax }}, K=P_{q i}-R \text { and } B=0 \\
\text { If } P_{q i}-R>C_{g \text { max }}, K=C_{g \max } \text { and } B=P_{q i}-R-K \\
\text { If } P_{q i}-R<0, K=0 \text { and } B=P_{q i}-R
\end{gathered}
$$

where $R$ is respiration rate, $C_{\text {gmax }}$ is the maximum rate of carbon used for growth, $K$ is growth, and $B$ is ballast. A conversion factor of 2.38 is applied to convert $B$, in grams of assimilated carbon, to $B_{g}$, grams of carbohydrate ballast (Equation 4):

$$
B_{g}=2.38 * B
$$

In their model, colony density $\left(\rho_{c}\right)$ is a function of cell density and water density as given by Equation 5, 


$$
\rho_{c}=\left(F * N * \rho_{c e l}\right)+\left[(1.0-F) \rho_{m u c}\right]
$$

where $F$ is the fraction of colony volume taken up by cells $(0.19), \rho_{c e l}$ is the density of a cell and $N$ is the number of cells in a colony. Mucilage density $\left(\rho_{m u c}\right)$ is estimated using density of the surrounding water (Equation 6).

$$
\rho_{\text {muc }}=\rho^{\prime}+0.7 \mathrm{~kg} / \mathrm{m}^{3}
$$

Changes in cell density based on changes in ballast are calculated by Equation 7,

$$
\Delta \rho_{c e l}=\frac{B_{g} C_{c e l}}{V_{c e l}}
$$

where $C_{c e l}$ is cell carbon content and $V_{c e l}$ is cell volume. This is translated to changes in colony density by Equation 8,

$$
\Delta \rho_{c}=\frac{\mathrm{N} * \mathrm{~V}_{\text {cel }} * \Delta \rho_{c e l}}{\frac{4}{3} \pi r^{3}}
$$

where $r$ is colony radius. As in Equation 3 from Kromkamp and Walsby (1990), settling velocity is calculated using Stokes' law (Equation 9).

$$
v=\frac{2 g r^{2}\left(\rho_{c}-\rho\right)}{9 \phi n}
$$

Howard et al. (1996) also defined a "turbulent mixed layer" in the surface layer of the model in which colonies are assumed to move with the speed of the surrounding water. This speed is calculated based on wind speed, and direction of colony movement is found with a random-walk routine. 
A model by Visser et al. (1997) was similar to the Kromkamp and Walsby (1990) model but included new treatments of photoinhibition and density change after dark. Instead of using a Michaelis-Menten equation for the relationship between cell density change and photon irradiance (as in the earlier study), they developed an irradiance-response curve based on laboratory experiments to better represent photoinhibition at high irradiance values. During periods when irradiance is higher than a compensation value $\left(I_{c}\right)$ the rate of density change is found using Equation 10,

$$
\frac{d \rho}{d t}=\left(\frac{N_{0}}{60}\right) I e^{-I / I_{0}}+c
$$

where $N_{0}$ is a regression coefficient, $c$ is the rate of density change when $I=0$, and $I_{0}$ is the light intensity corresponding to the maximum density. Additionally, they modeled the density decrease in the dark as a function of cell density rather than previously experienced irradiance. This is shown by Equation 11,

$$
\frac{d \rho}{d t}=f_{1} \rho_{i}+f_{2}
$$

where $\rho_{i}$ is the cell density at the end of the preceding light period, and $f_{1}$ and $f_{2}$ are regression coefficients.

Wallace and Hamilton (1999) made a contribution to these earlier models by adding a response time that begins after light intensity changes and lasts until the change in density with increasing irradiance becomes constant. They modified the 
equation used in Kromkamp and Walsby (1990), Equation 1, by adding an exponential decay term and neglecting the previous irradiance term (Equation 12).

$$
\frac{d \rho}{d t}=\left(c_{1} \frac{I}{K_{I}+I}-c_{3}\right)\left(1-e^{-t / \tau_{r}}\right)
$$

Here, $t$ is time since irradiance began and $\tau_{r}$ is the response time. They concluded that 20 minutes is generally an appropriate response time for models. However, the length of the irradiance time relative to the response time is important. They calculated density decrease using the second two terms of Equation 1 with modified coefficients (Equation 13).

$$
\frac{d \rho}{d t}=-c_{2} I_{a}-c_{3}
$$

Later studies made use of the buoyancy regulation equations (Table 1-2) from these earlier studies to model cyanobacteria movement (e.g. Easthope and Howard, 1999; Walsby, 2005; Chien et al., 2013; Yao et al., 2017). Some combined cyanobacteria buoyancy regulation with hydrodynamic models (e.g. Olsen et al., 2000; Wallace and Hamilton, 2000; Wallace et al., 2000; Bonnet and Poulin, 2002; Rabouille et al., 2003; Hedger et al., 2004; Rabouille and Salençon, 2005; Rabouille et al., 2005; Guven and Howard, 2006; Chen et al., 2009; Aparicio Medrano et al., 2013; Ndong et al., 2017; Wang et al., 2017; Zhu et al., 2018). Many models assume a single volume for all simulated colonies; however, some include the natural variation in colony size in a waterbody by assuming a distribution of colony diameters (e.g. Chien et al., 2013, Wang et al., 2017). 
Table 1-2. Equations used in studies and models of cyanobacteria vertical migration

\begin{tabular}{|c|c|c|}
\hline Equations & & Parameters \\
\hline \multicolumn{3}{|l|}{ Kromkamp and Walsby (1990) } \\
\hline $\begin{array}{l}\frac{d \rho}{d t}=c_{1}\left(\frac{I}{K_{I}+I}\right)-c_{2} I_{a}-c_{3} \\
v=\frac{2 g r^{2}\left(\rho_{c}-\rho^{\prime}\right) A}{9 \phi n}\end{array}$ & $\begin{array}{l}(1) \\
(2)\end{array}$ & $\begin{array}{l}c_{1}=0.132 \mathrm{~kg} \mathrm{~m}^{-3} \mathrm{~min}^{-1} \\
c_{2}=1.67 \times 10^{-5} \mathrm{~kg} \mathrm{~m}^{-3} \mathrm{~min}^{-1}\left(\mu \mathrm{mol} \mathrm{m} \mathrm{s}^{-2}\right)^{-1} \\
c_{3}=0.023 \mathrm{~kg} \mathrm{~m}^{-3} \mathrm{~min}^{-1} \\
K_{I}=25 \mu \mathrm{mol} \mathrm{m} \mathrm{s}^{-1}\end{array}$ \\
\hline \multicolumn{3}{|l|}{ SCUM96, Howard et al. (1996) } \\
\hline $\begin{array}{l}\rho_{c}=\left(F * N * \rho_{c e l}\right)+\left[(1.0-F) \rho_{m u c}\right] \\
\rho_{m u c}=\rho^{\prime}+0.7 \mathrm{~kg} / \mathrm{m}^{3} \\
\Delta \rho_{c e l}=\frac{B g C_{c e l}}{V_{c e l}} \\
\Delta \rho_{c}=\frac{\mathrm{N} * V_{c e} * \Delta \rho_{c e l}}{\frac{4}{3} \pi r^{3}} \\
v=\frac{2 g r^{2}\left(\rho_{c}-\rho^{\prime}\right)}{9 \phi n}\end{array}$ & $\begin{array}{l}\text { (5) } \\
(6) \\
(7) \\
(8) \\
(9)\end{array}$ & $\begin{array}{l}F=0.19 \\
N=12,032\end{array}$ \\
\hline \multicolumn{3}{|l|}{ Visser et al. (1997) } \\
\hline $\begin{array}{l}I \geq I_{c}, \frac{d \rho}{d t}=\left(\frac{N_{0}}{60}\right) I e^{-I / I_{0}}+c \\
I<I_{c}, \frac{d \rho}{d t}=f_{1} \rho_{i}+f_{2}\end{array}$ & (11) & $\begin{array}{l}I_{c}=10.9 \mu \mathrm{mol} \mathrm{m} \mathrm{m}^{-2} \mathrm{~s}^{-1} \\
N_{0}=0.0945 \mathrm{~kg} \mathrm{~m}^{-3} \mu \mathrm{mol}^{-1} \mathrm{~m}^{2} \\
I_{0}=277.5 \mu \mathrm{mol} \mathrm{m}^{-2} \mathrm{~s}^{-1} \\
c=-0.0165 \mathrm{~kg} \mathrm{~m}^{-3} \mathrm{~min}^{-1} \\
f_{1}=-9.49 \times 10^{-4} \mathrm{~min}^{-1} \\
f_{2}=0.984 \mathrm{~kg} \mathrm{~m}^{-3} \mathrm{~min}^{-1}\end{array}$ \\
\hline \multicolumn{3}{|l|}{ Wallace and Hamilton (1999) } \\
\hline $\begin{array}{l}I>0, \frac{d \rho}{d t}=\left(c_{1} \frac{I}{K_{I}+I}-c_{3}\right)\left(1-e^{-t / \tau_{r}}\right) \\
I=0, \frac{d \rho}{d t}=-c_{2} I_{a}-c_{3}\end{array}$ & $(12)$ & $\begin{array}{l}c_{1}=0.0427 \mathrm{~kg} \mathrm{~m}^{-3} \mathrm{~min}^{-1} \\
c_{2}=1.67 \times 10^{-5} \mathrm{~kg} \mathrm{~m}^{-3} \mathrm{~min}^{-1}\left(\mu \mathrm{mol} \mathrm{m}^{-2} \mathrm{~s}^{-1}\right)^{-1} \\
c_{3}=4.6 \times 10^{-6} \mathrm{~kg} \mathrm{~m}^{-3} \mathrm{~min}^{-1} \\
K_{I}=530 \mu \mathrm{mol} \mathrm{m} \mathrm{m}^{-2} \mathrm{~s}^{-1} \\
\tau_{r}=20 \mathrm{~min}\end{array}$ \\
\hline \multicolumn{3}{|l|}{ Belov and Giles (1997) } \\
\hline$v_{s}(z, t)=V_{0} e^{-k(h-z)} \cos (\omega t)$ & (14) & $\begin{array}{l}V_{0}=0.408 m_{d a y^{-1}} \\
k=0.1 m^{-1} \\
\omega=2 \pi d a y^{-1}\end{array}$ \\
\hline \multicolumn{3}{|l|}{ Serizawa et al. (2008) } \\
\hline $\begin{array}{l}V(t, z)=V_{m}\left\{F(t, z)-F_{0}\right\} \\
F(t, z)=\int_{0}^{\infty} \mu(t-\tau, z) e^{-k \tau} d \tau\end{array}$ & $\begin{array}{l}(15) \\
(16)\end{array}$ & $\begin{array}{l}V_{m}=250 \mathrm{mday}^{-1} \\
F_{0}=0.1 \\
k=3 \text { day }^{-1}\end{array}$ \\
\hline \multicolumn{3}{|l|}{ CAEDYM, Hipsey et al. (2007) } \\
\hline $\begin{array}{l}I>0, \frac{d \rho}{d t}=c_{1}\left(1-e^{-I / I_{K}}\right)-c_{3} \\
I=0, \frac{d \rho}{d t}=-c_{3}\end{array}$ & $\begin{array}{l}(30) \\
(31)\end{array}$ & $\begin{array}{l}c_{1}=0.124 \mathrm{~kg} \mathrm{~m}^{-3} \min ^{-1_{\mathrm{a}}} \\
c_{3}=0.023 \mathrm{~kg} \mathrm{~m}^{-3} \mathrm{~min}^{-1_{\mathrm{a}}} \\
K_{I}=130 \mu \mathrm{mol} \mathrm{m} \mathrm{s}^{-1} \mathrm{~s}^{-a}\end{array}$ \\
\hline
\end{tabular}

a Values used in Chung et al. (2014) 
The above models require knowledge of a colony's density history and are thus better suited for Lagrangian-type models that track simulated particles. Other models of cyanobacteria vertical migration have been designed for a Eulerian framework that treats plankton as a continuum. Belov and Giles (1997) developed one such model based on principles of light-dependent buoyancy regulation. However, they used a predetermined colony velocity rather than the dynamic settling velocity approach of the above models. Their velocity model (Equation 14) incorporates light changes due to the daily solar cycle as well as the depthdependent effect of light extinction in a waterbody. In this way, it assumes the density change and movement of cyanobacteria in response to light without requiring information about actual light or colony density.

$$
v_{s}(z, t)=V_{0} e^{-k(h-z)} \cos (\omega t)
$$

Here, $v_{s}$ is the velocity of a colony at depth $z$ and time $t, V_{0}$ is the maximum velocity of a colony, $k$ is the light attenuation coefficient of the waterbody, $h$ is the depth of the waterbody, and $\omega$ is the frequency of the daily light cycle.

Serizawa et al. (2008) also created a model idealized for a continuum approach. Their model incorporates the light and nutrient histories that cyanobacteria colonies would have experienced in each model location. As in Belov and Giles (1997), they define a velocity, $V(t, z)$, in time and space (Equation 15).

$$
V(t, z)=V_{m}\left\{F(t, z)-F_{0}\right\}
$$


This includes two constants, $V_{m}$ and $F_{0}$, which are the velocity scale factor and neutral buoyancy ballast factor, respectively. Changes in velocity are determined by the ballast factor, $F(t, z)$, which represents the cumulative effect of past growth rates, $\mu$, at a particular depth, $z$ (Equation 16). This assumes a relationship between cyanobacteria growth kinetics and migration velocity based on the idea that buoyancy regulation and growth respond to similar inputs.

$$
F(t, z)=\int_{0}^{\infty} \mu(t-\tau, z) e^{-k \tau} d \tau
$$

The exponential decay factor in Equation 16 gives less weight to growth rates experienced further in the past. The variable $\tau$ represents time before present and the constant $k$ is the reciprocal of decay time.

\subsubsection{Zooplankton}

Unlike phytoplankton, zooplankton cannot make their own food. Although plankton typically refers to non-motile organisms, some zooplankton can swim up and down in the water column (Ringelberg, 2010). This is frequently observed in cladoceran and copepod species (Ibid.) as well as euphausiids, also known as krill (Andersen and Nival, 1991) and opossum shrimp (Boscarino et al., 2009). When migration patterns follow a diurnal pattern, they are called diel vertical migration, or DVM (Cohen and Forward, 2009). A large portion of research has focused on DVM of the cladoceran Daphniagenus in particular (Ringelberg, 1999).

As in cyanobacteria, vertical migration in zooplankton is thought to be an adaptive strategy that allows organisms to benefit from resources found in either 
the surface or bottom layers of a waterbody. The surface layers are generally more favorable to zooplankton growth and reproduction due to warmer temperatures and higher food concentrations. However, this region is typically brighter than lower layers and leaves zooplankton more vulnerable to visual predators, such as fish. According to the prevailing theory, zooplankton migrate to deep, darker waters during the day to avoid visual predators and return to surface layers after dark to feed and experience warmer temperatures (Han and Straškraba, 1998).

Studies on zooplankton DVM often classify factors affecting migration as either proximate or ultimate (e.g. Cohen and Forward, 2009; Ringelberg, 1999; Ringelberg, 2010; Ringelberg and Van Gool, 2003; Rinke and Petzoldt, 2008; Williamson et al., 2011). According Ringelberg and Van Gool, (2003), proximate factors represent how an organism migrates, while ultimate factors are the reasons why it migrates. They assert that light is the primary proximate factor affecting zooplankton DVM and that the presence of predators, food abundance, and temperature are secondary proximate factors. The primary ultimate factor affecting zooplankton DVM is generally believed to be predator avoidance (Cohen and Forward, 2009; Ringelberg and Van Gool, 2003; Rinke and Petzoldt, 2008). In a meta-analysis of 24 field studies on Daphnia, Dodson (1990) found a significant relationship between Secchi depth and vertical migration amplitude. They also found that regression residuals were significantly correlated with moon phase. Field and laboratory experiments suggest that zooplankton respond to light 
in three ways: by migrating to stay at a certain light intensity, by initiating movement after a threshold intensity is reached, or by initiating movement when a threshold rate of change of light is reached (see Cohen and Forward [2009] for a review). In laboratory experiments on Daphnia, Van Gool and Ringelberg (2003) observed that the relationship between vertical migration velocity and relative change in light was positively correlated with fish kairomones, food concentration, and temperature. In a field study of zooplankton in a lake in Uganda, Semyalo et al. (2009) observed DVM in several species of zooplankton and found that water transparency was positively correlated with migration amplitude of the calanoid copepod Tropocyclops galeboides. Larsson and Lampert (2012) conducted laboratory experiments on Daphnia pulicaria both with and without predatory fish present. They observed that when predators were not present, D. pulicaria's vertical position was dependent primarily on food concentration, followed by temperature and dissolved oxygen. When predators were present, D. pulicaria was observed to forgo food and temperature preferences, but not dissolved oxygen preference. In field experiments in Lake Huron, Nowicki et al. (2017) observed DVM in eight cladoceran and copepod zooplankton species and identified predator abundance as the most common explanatory variable. In some species, Secchi depth and temperature were also important factors.

In several studies, zooplankton DVM was modeled by a fitness optimization function. Fiksen (1997) modeled Daphnia DVM by optimizing a 
reproduction/predation optimization function, where predation was a function of fish density and light at depth and reproduction was a function of depth. They then solved this function for the optimal depth of zooplankton. A similar model for the copepod Calanus finmarchicus was developed by Fiksen and Carlotti (1998). Han and Straškraba (1998) developed a model that minimizes the change in sensed predation pressure by zooplankton. In this model, sensed predation pressure is a function of light, food concentration, temperature, and predation pressure at depth. An energy gain/predation optimization model for the krill species Meganyctiphanes norvegica was developed by Tarling et al. (2000). Results of this model suggested that food and predation were the primary drivers of DVM, while temperature had a minor effect. De Robertis (2002) and Kessler and Lampert (2004) developed similar models that predict the timing of DVM based on light intensity at depth. Jensen et al. (2006) modeled the migration of lake trout, ciscoes, and opossum shrimp in Lake Superior by optimizing feeding rate potential, which is a function of light intensity at depth and prey abundance.

Other studies have produced mechanistic models of zooplankton DVM that do not use optimization functions (Table 1-3). Dodson (1990) developed a simple linear regression model of Daphnia vertical movement as a function of Secchi depth based on data gathered from studies of different lakes in the United States and Europe. Their model predicts the migration amplitude $(M)$ or diel change in average 
population depth, based on the clarity of the water as quantified by Secchi depth, $D_{S}$ (Equation 17).

$$
M=1.409 D_{s}-0.317
$$

This model predicts that Daphnia will travel greater vertical distances during a day when water is more clear.

Table 1-3. Equations used in studies and models of zooplankton light-dependent diel vertical migration

\begin{tabular}{|c|c|c|}
\hline \multicolumn{2}{|l|}{ Equation } & Parameters \\
\hline \multicolumn{3}{|l|}{ Dodson (1990) } \\
\hline$M=1.409 D_{s}-0.317$ & (17) & \\
\hline \multicolumn{3}{|l|}{ Andersen and Nival (1991) } \\
\hline $\begin{array}{l}w=w_{m} l_{I 1} l_{I 2} \\
I_{z}>I_{e}, \quad l_{I 1}=\left[\frac{I_{z}}{I_{s}}\left(\frac{I_{e}-I_{z}}{I_{e}-I_{s}}\right)^{\frac{I_{e}-I_{s}}{I_{s}}}\right]^{\alpha} \\
l_{\text {var }}=\frac{100\left(I_{t}-I_{t-\Delta t}\right)}{I_{t}} \\
\left|I_{\text {var }}\right| \leq I_{v}, l_{I 2}=-\left(I_{v a r}^{3}\right) \\
I_{\text {var }}>I_{v}, l_{I 2}=-\left(I_{v}^{3}\right) \\
I_{v a r}<-I_{v}, l_{I 2}=I_{v}^{3} \\
w=R w_{f}\left(1-e^{-k_{f}(P+Z)}\right)\end{array}$ & $\begin{array}{l}(21) \\
(22) \\
(23) \\
(24)\end{array}$ & $\begin{array}{l}w_{m}=94 \mathrm{~m} \mathrm{day}^{-1} \\
I_{e}=10 \mu \mathrm{mol} \mathrm{m} \mathrm{m}^{-2} \mathrm{~s}^{-1} \\
I_{s}=0.1 \mu \mathrm{mol} \mathrm{m} \mathrm{m}^{-2} \mathrm{~s}^{-1} \\
\alpha=0.012 \\
I_{v}=3 \% \\
R=-1 \mathrm{or} 1 \\
w_{f}=2400 \mathrm{~m} \mathrm{day}^{-1} \\
k_{f}=1.2 \mu \mathrm{mol} \mathrm{N}^{-1}\end{array}$ \\
\hline \multicolumn{3}{|l|}{ Richards et al. (1996) } \\
\hline $\begin{array}{l}S(t)>R, W(z, t)=g(z, t) * \min \left(W_{d}, \frac{\beta}{1+k \beta}(S(t)-R)\right) \\
S(t)<-R, W(z, t)=g(z, t) * \max \left(-W_{u}, \frac{\beta}{1+k \beta}(S(t)+R)\right) \\
S(t)=\frac{1}{I_{0}} \frac{\partial I_{0}}{\partial t} \\
0<t \leq 12, g(z, t)= \begin{cases}1 & \text { if } 0 \leq z \leq H-\gamma \\
\cos \left(\frac{\pi}{2 \gamma}(z-H+\gamma)\right) & \text { if } H-\gamma \leq z \leq H\end{cases} \\
0<t \leq 12, g(z, t)= \begin{cases}1 & \text { if } \gamma \leq z \leq H \\
\sin \left(\frac{\pi}{2 \gamma} z\right) & \text { if } 0 \leq z \leq \gamma\end{cases} \end{array}$ & $(28)$ & $\begin{array}{l}R=0.025 \mathrm{~min}^{-1} \\
W_{d}=0.2 \mathrm{~cm} \mathrm{~s}^{-1} \\
W_{u}=0.2 \mathrm{~cm} \mathrm{~s}^{-1} \\
\beta=25 \mathrm{~m} \\
\gamma=2 \mathrm{~m}\end{array}$ \\
\hline
\end{tabular}


Based on data from previous studies, Andersen and Nival (1991) modeled DVM of the krill Meganyctiphanes norvegica as a function of light intensity, rate of irradiance change, and food abundance. In their model, migration speed ( $w)$ is primarily a function of absolute light intensity and irradiance change (Equation 18).

$$
w=w_{m} l_{I 1} l_{I 2}
$$

Here, $w_{m}$ is the basic migration speed, $l_{I 1}$ is the absolute light intensity factor, and $l_{I 2}$ is the irradiance change factor. When absolute light intensity $\left(I_{z}\right)$ is less than a threshold intensity $\left(I_{e}\right), I_{I 1}$ is calculated with Equation 19,

$$
l_{I 1}=\left[\frac{I_{z}}{I_{s}}\left(\frac{I_{e}-I_{z}}{I_{e}-I_{S}}\right)^{\frac{I_{e}-I_{S}}{I_{S}}}\right]^{\alpha}
$$

where $I_{S}$ is the optimal light intensity for migration speed and $\alpha$ is the shape factor of the irradiance-response curve. When $I_{z}$ is greater than $I_{e}, I_{I 1}$ is zero. The effect of irradiance change on migration speed depends on the value of relative irradiance variation $\left(I_{\text {var }}\right)$ relative to an irradiance threshold above which migration speed does not change $\left(I_{v}\right)$. Equation 20 is used to calculate $I_{v a r}$, where $I_{t}$ is the surface irradiance at time $t$.

$$
l_{v a r}=\frac{100\left(I_{t}-I_{t-\Delta t}\right)}{I_{t}}
$$

The value of $I_{I 2}$ is then calculated using Equations 21 - 23, resulting in positive (upward) velocities at dusk and negative (downward) velocities at dawn.

$$
\left|I_{\text {var }}\right| \leq I_{v}, l_{I 2}=-\left(I_{v a r}^{3}\right)
$$




$$
\begin{gathered}
I_{v a r}>I_{v}, l_{I 2}=-\left(I_{v}^{3}\right) \\
I_{v a r}<-I_{v}, l_{I 2}=I_{v}^{3}
\end{gathered}
$$

When organisms are ascending through the water column and encounter a food gradient, their speed is determined by Equation 24,

$$
w=R w_{f}\left(1-e^{-k_{f}(P+Z)}\right)
$$

where $R$ is randomly 1 or $-1, w_{f}$ is the maximum food-dependent speed, $k_{f}$ is the shape factor for the food-dependent velocity curve, and the sum of $P$ and $Z$ represents prey concentration (phyto- and zooplankton in this case).

Richards et al. (1996) developed three models of zooplankton DVM based on absolute light intensity, rate of change of light intensity, and relative rate of change of light intensity. In their model based on relative rate of change of light $(S)$, velocity as a function of time and space, $W(z, t)$, is determined using Equations 25 and 26.

$$
\begin{aligned}
& S(t)>R, W(z, t)=g(z, t) * \min \left(W_{d}, \frac{\beta}{1+k \beta}(S(t)-R)\right) \\
& S(t)<-R, W(z, t)=g(z, t) * \max \left(-W_{u}, \frac{\beta}{1+k \beta}(S(t)+R)\right)
\end{aligned}
$$

The decision for which equation to use is determined by the sign and magnitude of $S(t)$ relative to the rheobase $(R)$ which is a threshold for zooplankton response to light. $S(t)$ is calculated with Equation 27, where $I_{0}$ is light intensity at the water surface. 


$$
S(t)=\frac{1}{I_{0}} \frac{\partial I_{0}}{\partial t}
$$

A positive value of $S(t)$ indicates that light is increasing and induces a positive (downward) motion, while a negative value indicates that light is decreasing and induces negative (upward) movement. If the magnitude of $S(t)$ is less than $R$, migration speed is set to zero. In both equations, the speed is limited by maximum downward and upward speeds ( $W_{d}$ and $W_{u}$, respectively). When light intensity at depth, $I(z, t)$, is less than $0.01 \%$ of the daily maximum surface intensity, migration velocity is set to zero. In Equations 25 and 26, $a$ is light attenuation and $\beta$ is a parameter of zooplankton light sensitivity. This term causes migration speeds to decrease when water is less clear. The first term in Equations 25 and 26, $g(z, t)$, determines movement in the regions near the water surface and the bed. This term varies based on time of day such that between midnight and noon (when organisms are assumed to be moving downward), motion is not affected when organisms are not close to the bed (Equation 28). In the region near the bed, determined by the parameter $\gamma$, speed decreases until it is zero at the bed. Between noon and midnight, motion is uninhibited from the bed to near the surface, where is decreases until it is zero at the surface (Equation 29).

$$
\begin{aligned}
& 0<t \leq 12, \quad g(z, t)= \begin{cases}1 & \text { if } 0 \leq z \leq H-\gamma \\
\cos \left(\frac{\pi}{2 \gamma}(z-H+\gamma)\right) & \text { if } H-\gamma \leq z \leq H\end{cases} \\
& 0<t \leq 12, \quad g(z, t)= \begin{cases}1 & \text { if } \gamma \leq z \leq H \\
\sin \left(\frac{\pi}{2 \gamma} z\right) & \text { if } 0 \leq z \leq \gamma\end{cases}
\end{aligned}
$$


Here, $z$ is the vertical distance from the water surface to a depth in the water column and $H$ is the total depth of the water column.

More complicated models of zooplankton DVM include the influence of other factors besides light. Rinke and Petzoldt (2008) modeled Daphnia DVM as a function of food concentration, light intensity, biomass of predatory fish, and life stage. In their model of Daphnia DVM, Ringelberg (2010) included constant, high-amplitude, low-frequency oscillations and modeled light-induced movement as functions of relative light change, fish kairomone concentration, food concentration, and temperature.

\subsubsection{Other Models}

Vertical migration of phytoplankton and zooplankton is included in several widely used numerical water-quality models. Descriptions of these models are given below.

\section{PROTECH}

PROTECH is a commonly used model of phytoplankton dynamics (Trolle et al., 2012). Up to ten species of phytoplankton from a library of over 100 species can be modeled at one time (Mooij et al., 2010). Species that regulate their buoyancy move up or down a specified number of model cells based on light at depth (Reynolds et al., 2001). However, the model has a minimum timestep of one day, so it cannot simulate vertical migration within a 24-hour period. 


\section{CAEDYM}

CAEDYM is a widely used numerical water-quality model and is often coupled with the one-dimensional lake model DYRESM (Trolle et al., 2012). In CAEDYM, vertical migration of cyanobacteria is based on the theory and equations presented in Kromkamp and Walsby (1990) and is a function of light intensity (Hipsey et al., 2007). Rate of density change with irradiance is given by Equation 30,

$$
\frac{d \rho}{d t}=c_{1}\left(1-e^{-I / I_{K}}\right)-c_{3}
$$

which is modified from Equation 1 by the addition of an exponential light response term. Here, $I_{K}$ is the half saturation constant for light-dependent density change. Alternatively, rate of density change can be modeled as a function of internal carbon store. Rate of density change in the dark is not a function of previous irradiance as in Equation 1, but is based only on a constant (Equation 31):

$$
\frac{d \rho}{d t}=-c_{3}
$$

In the case of dinoflagellates, chlorophytes, and cryptophytes migration velocity is modeled as a function of irradiance and internal nitrogen stores.

\section{BELAMO}

BELAMO is a one-dimensional, biogeochemical lake model (Mieleitner and Reichert, 2006). Zooplankton movement is modeled as a diffusive process. Diffusion coefficients are based on dissolved oxygen in each layer in such a way that zooplankton move towards layers with higher dissolved oxygen. 


\section{$\underline{\text { SALMO }}$}

SALMO is a one-dimensional lake ecosystem model (Mooij et al., 2010). In this model, zooplankton migrate from the hypolimnion to the epilimnion during stratification (Recknagel et al., 2008). Available model documentation does not explain the mechanism by which this happens. Zooplankton vertical migration speed is a constant, user-defined variable in the model. 


\section{Chapter 2: Model Development}

\subsection{Overview}

The purpose of this work is to develop a model of plankton vertical migration that can be incorporated into the hydrodynamic and water-quality model CE-QUALW2. Several different model approaches were investigated, including predefined velocity and dynamically calculated velocity based on light. These approaches were applied to models of cyanobacteria and zooplankton using both continuum and particle-tracking frameworks.

In the continuum framework, plankton was modeled as a mass concentration that is homogeneous within each model grid cell. The governing equation for transport of plankton in a continuum framework is the advection-diffusion equation. For a one-dimensional (vertical) model in a quiescent waterbody with constant horizontal area with depth, the governing mass balance equation is given by Equation 32,

$$
\frac{d c}{d t}+\frac{\partial c v_{p}}{\partial z}=\frac{\partial}{\partial z}\left[D_{z}\left(\frac{\partial c}{\partial z}\right)\right]-r
$$

where $c$ is plankton concentration, $t$ is time, $z$ is depth in the waterbody, $v_{p}$ is the vertical migration velocity of the organism or colony, $D_{z}$ is the vertical diffusion coefficient, and $r$ is a source-sink term for population growth and loss.

Cyanobacteria or zooplankton concentration can be solved for at each point in time and space using an appropriate numerical scheme. 
For the continuum framework, an upwind numerical scheme with no-flux boundary conditions was used to solve Equation 32:

$$
\begin{aligned}
& c_{i}^{n+1}=\frac{\mathrm{D}_{\mathrm{z}} \Delta t}{\Delta \mathrm{z}^{2}}\left(c_{i+1}^{n}-2 c_{i}^{n}+c_{i-1}^{n}\right)-\frac{\Delta t}{\Delta z}\left(v_{p_{i}}^{n} c_{i}^{n}-v_{p_{i-1}}^{n} c_{i-1}^{n}\right)+\left(\mu_{n e t} \Delta t+1\right) c_{i}^{n}, \quad v_{p_{i}}^{n}>0 \\
& c_{i}^{n+1}=\frac{\mathrm{D}_{\mathrm{z}} \Delta t}{\Delta \mathrm{z}^{2}}\left(c_{i+1}^{n}-2 c_{i}^{n}+c_{i-1}^{n}\right)-\frac{\Delta t}{\Delta z}\left(v_{p_{i+1}}^{n} c_{i+1}^{n}-v_{p_{i}}^{n} c_{i}^{n}\right)+\left(\mu_{n e t} \Delta t+1\right) c_{i}^{n}, \quad v_{p}^{n}<0
\end{aligned}
$$

In Equations 33 and 34, $\Delta z$ is the model grid spacing and the subscript $i$ refers to the model grid cell of interest (Figure 2-1). The superscripts refer to time in the simulation, where time $n+1$ is one timestep in the future from time $n$, and $\Delta t$ is the model timestep. The source-sink term of Equation 32 is represented by $\mu_{n e t}$ and includes the effects of population growth, mortality, excretion, and respiration.

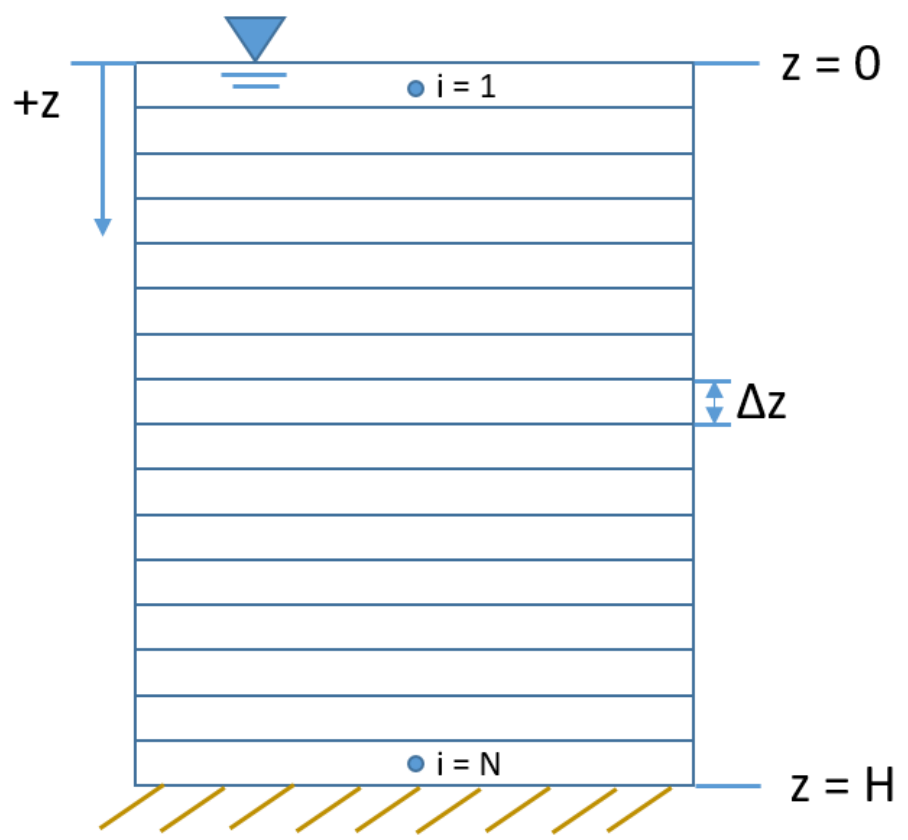

Figure 2-1 Layout of model grid used in preliminary models 
In a particle-tracking framework, the location of an organism or colony, represented by a particle, is calculated at each timestep rather than calculating the concentration of plankton in a particular location. Equation 35 gives a numerical solution for a model of plankton depth, $z_{p}$ (Mascarenhas and Trento, 2006):

$$
z_{p_{i}}^{n+1}=z_{p_{i}}^{n}+v_{p_{i}}^{n} \Delta t+R \sqrt{6 D_{z_{i}}^{n} \Delta t}
$$

where the subscript $i$ refers to the particle of interest, the superscript $n$ refers to the time in the simulation, and $\Delta t$ is the model timestep. The third term on the righthand side includes a random number, $R$, from a uniform distribution between -1 and 1 , and represents the variation in motion among particles due to diffusion. Particles that were predicted to move past the bed $(\mathrm{z}=\mathrm{H})$ during a displacement were instead assigned a location equal to one half of a grid cell width above the bed. Particles that were predicted to move past the surface $(\mathrm{z}=0)$ during a displacement were assigned a location equivalent to the surface.

In order to compare results from models in the particle-tracking framework to those from models in the continuum framework, concentration predicted in the particle-tracking framework was computed using Equation 36 after summing the number of particles over a control volume corresponding to each model grid cell.

$$
c_{i}^{n}=\frac{P_{i}^{n} m}{\Delta z B x}
$$


Here, $P_{i}^{n}$ is the total number of particles located between depths $(i-1) \Delta z$ and $i \Delta z$ at time $n, m$ is the mass of one particle, and $B$ and $x$ are the lateral and longitudinal dimensions of the model grid, respectively.

\subsection{Cyanobacteria}

\subsubsection{Predefined Velocity}

A simple way to model the vertical movement of cyanobacteria is to assume a velocity function for colonies based on knowledge of their typical movement. Because cyanobacteria vertical migration is due to buoyancy regulation, which is dependent on light, a velocity function that represents changes in light is a logical choice (Table 2-1 \& Table 2-2).

If cyanobacteria colonies are assumed to migrate vertically on a daily cycle, an equation for colony velocity as a function of time can be used (Equation 37).

$$
v_{p}(t)=A \frac{2 \pi}{86,400 s} \cos \left(\frac{2 \pi}{86,400 s} t+\phi\right)
$$

Here, $A$ is migration amplitude and the period is assumed to be one day $(86,400$ seconds). The value of the phase $(\phi)$ depends on the initial location of colonies. For example, if $t=0$ in the simulation corresponds to midnight and colonies are assumed to be at the bottom at that time, the value is $\frac{\pi}{2}$ with positive velocity corresponding to downward movement (Figure 2-1). 
Table 2-1, Predefined velocity models used in continuum framework

\begin{tabular}{|l|l|}
\hline \multicolumn{1}{|c|}{ Model } & \multicolumn{1}{|c|}{ Equations } \\
\hline \multirow{2}{*}{$\begin{array}{l}\text { Time-varying } \\
\text { velocity }\end{array}$} & $\begin{array}{l}v_{p_{i}}^{n}>0: \\
c_{i}^{n+1}=\frac{\mathrm{D}_{\mathrm{z}} \Delta t}{\Delta z^{2}}\left(c_{i+1}^{n}-2 c_{i}^{n}+c_{i-1}^{n}\right)-\frac{\Delta t}{\Delta z}\left(v_{p_{i}}^{n} c_{i}^{n}-v_{p_{i-1}}^{n} c_{i-1}^{n}\right)+\left(\mu_{n e t} \Delta t+1\right) c_{i}^{n} \\
v_{p}^{n}<0: \\
c_{i}^{n+1}=\frac{\mathrm{D}_{\mathrm{z}} \Delta t}{\Delta z^{2}}\left(c_{i+1}^{n}-2 c_{i}^{n}+c_{i-1}^{n}\right)-\frac{\Delta t}{\Delta z}\left(v_{p_{i+1}}^{n} c_{i+1}^{n}-v_{p_{i}}^{n} c_{i}^{n}\right)+\left(\mu_{n e t} \Delta t+1\right) c_{i}^{n}\end{array}$ \\
\hline $\begin{array}{l}\text { Belov \& Giles } \\
(1997)\end{array}$ & $v_{p}(t)=A \frac{2 \pi}{86,400 s} \cos \left(\frac{2 \pi}{86,400 s} t+\phi\right)$ \\
& $v_{p}(t, z)=\left\{\begin{array}{l}A \frac{2 \pi}{86,400 s} \cos \left(\frac{2 \pi}{86,400 s} t+\phi\right) e^{-\alpha(H-z)}, \\
A \frac{2 \pi}{86,400 s} \cos \left(\frac{2 \pi}{86,400 s} t+\phi\right),\end{array}\right.$ \\
\hline
\end{tabular}

Table 2-2 Predefined velocity models used in particle-tracking framework

\begin{tabular}{|c|l|}
\hline Model & \multicolumn{1}{|c|}{ Equations } \\
\hline Time-varying velocity & $z_{p_{i}}^{n+1}=z_{p_{i}}^{n}+v_{p_{i}}^{n} \Delta t+R \sqrt{6 D_{z_{i}}^{n} \Delta t}$ \\
& $v_{p}(t)=A \frac{2 \pi}{86,400 s} \cos \left(\frac{2 \pi}{86,400 s} t+\phi\right)$ \\
\hline Belov \& Giles (1997) & $v_{p}(t, z)=\left\{\begin{array}{l}A \frac{2 \pi}{86,400 s} \cos \left(\frac{2 \pi}{86,400 s} t+\phi\right) e^{-\alpha(H-z)}, \\
A \frac{2 \pi}{86,400 s} \cos \left(\frac{2 \pi}{86,400 s} t+\phi\right),\end{array}\right.$ \\
\hline
\end{tabular}

A slightly more complex approach is to assume a velocity function that is dependent on space as well as time, as in Belov and Giles (1997). Modifying Equation 14 to use the same notation as Equation 37 gives:

$$
v_{p}(t, z)= \begin{cases}A \frac{2 \pi}{86,400 s} \cos \left(\frac{2 \pi}{86,400 s} t+\phi\right) e^{-\alpha(H-z)}, & I_{0}>0 \\ A \frac{2 \pi}{86,400 s} \cos \left(\frac{2 \pi}{86,400 s} t+\phi\right), & I_{0} \leq 0\end{cases}
$$

Here, $\alpha$ is the light attenuation coefficient and $I_{0}$ is solar irradiance at the water surface. The addition of the exponential term gives colonies deeper in the water column higher speeds and responds to variations in water clarity when the light 
attenuation coefficient, $\alpha$, is variable. In the original study, the light attenuation coefficient was assumed to be constant and the exponential term was applied whether or not there was irradiance at the water surface. Here, the exponential term is only applied during the photoperiod so that the effects of water clarity are only included when there is sunlight present. During dark periods, the equation reduces to Equation 37.

\subsubsection{Dynamic Velocity}

The above approaches can predict cyanobacteria movement based on the observed tendency of colonies to migrate vertically on a daily cycle; however, they do not reflect the response of colonies to variations in solar irradiance. In order to capture this natural behavior, colony velocity was also calculated based on relationships between sunlight and cyanobacteria growth and colony density. Using this approach, the change in cyanobacteria colony density was computed based on the solar irradiance at the surface of the water and the colony's depth in the water column. This density was then used to solve for the colony's settling velocity via Stokes' law (Equation 2). Three different approaches were tested to model cyanobacteria buoyancy change: a model based on growth kinetics, the model from Visser et al. (1997), and a model that incorporates light response and calibration coefficients (Table 2-3 \& Table 2-4).

\section{Growth Kinetics Model}

Because cyanobacteria buoyancy regulation is controlled by the accumulation and depletion of photosynthetic products, changes in cell and colony 
density follow similar patterns to algal growth kinetics (Serizawa et al., 2008). In CE-QUAL-W2, the change in total mass of an algal population is the sum of increases due to growth and losses due to respiration, excretion, mortality, and zooplankton grazing. Growth rate is based on a maximum growth rate scaled by temperature, light, and available nutrients (Cole and Wells, 2018). The light-driven growth of cyanobacteria is the focus of the present study, so it was assumed that light was the only limiting factor for growth and zooplankton grazing was ignored. Assuming that the change in colony density can be calculated based on the net growth rate $\left(\mu_{n e t}\right)$, the change in cyanobacteria colony density $\left(\rho_{c}\right)$ is given by Equation 39 .

$$
\frac{\partial \rho_{c}}{\partial t}=\mu_{n e t} \rho_{c}
$$

The net growth rate for cyanobacteria in this case is given by Equation 40,

$$
\mu_{n e t}=\mu_{g, \max } F(I)-\mu_{r}-\mu_{e}-\mu_{m}
$$

where $\mu_{g, \max }$ is the maximum growth rate, $\mu_{r}$ is the respiration rate, $\mu_{e}$ is the excretion rate, and $\mu_{m}$ is the mortality rate of the cyanobacteria species. A function of light, $F(I)$, scales the maximum growth rate and is given by the Steel equation, which accounts for photoinhibition at high irradiance values (Equation 41).

$$
F(I)=\frac{I}{I_{s}} e^{-\frac{I}{I_{s}}+1}
$$


Here, $I$ is irradiance at the location of interest and $I_{S}$ is the saturating light intensity for the cyanobacteria species. Irradiance at the depth of the colony is found with Equation 42,

$$
I(z)=(1-\beta) I_{0} e^{-\alpha z}
$$

where $\beta$ is the fraction of solar irradiance absorbed at the water surface, and $\alpha$ is the light attenuation coefficient.

In the particle-tracking framework, the density of each cyanobacteria colony was calculated along with its location at each timestep. Solving Equation 39 for the density of colony $i$ at time $n+1$ gives

$$
\rho_{c_{i}}^{n+1}=\rho_{c_{i}}^{n} \mu_{n e t_{i}}^{n} \Delta t+\rho_{c_{i}}^{n}
$$

where $\mu_{n e t_{i}}^{n}$ is calculated at the location of the colony at time $n$. The velocity of the colony was then found using Stokes' law (Equation 2) and substituted into Equation 35 to determine the colony's new position. Each colony was assumed to have the same radius throughout the simulation and the total number of colonies did not change. Cyanobacteria concentration in each model grid cell was found with Equation 36.

Applying a light-driven density change to colonies in a continuum framework requires a different approach than in a particle-tracking framework. In the particletracking framework, colonies are followed throughout the simulation and their densities are cumulative from the start of the simulation. In a continuum 
framework, there is no distinction between colonies in the water and therefore no way to track each colony's density change over time. To overcome this, colony density change was determined for each model grid cell using Equation 43, with the subscript $i$ now referring to the grid cell of interest rather than the particle of interest. The colony velocity for each grid cell was found using Stokes' law as in the particle-tracking framework (Equation 2).

The numerical scheme for solving Equation 32 differs from Equations 33 and 34 because velocities in neighboring grid cells can have opposite directions (Equation 44).

$c_{i}^{n+1}=\frac{\mathrm{D}_{\mathrm{z}} \Delta t}{\Delta z^{2}}\left(c_{i+1}^{n}-2 c_{i}^{n}+c_{i-1}^{n}\right)-\frac{\Delta t}{\Delta z}\left(\left|v_{p_{i}}^{n} c_{i}^{n}\right|-v_{p_{B}}^{n} c_{i+1}^{n}-v_{p_{T}}^{n} c_{i-1}^{n}\right)+\left(\mu_{n e t} \Delta t+1\right) c_{i}^{n}(44)$ Here, $v_{c_{B}}$ and $v_{c_{T}}$ are the velocities of colonies entering grid cell $i$ from above and below, respectively (Equations $45 \& 46)$.

$$
\begin{aligned}
& v_{p_{B}}^{n}= \begin{cases}v_{p_{i+1}}^{n}, & v_{p_{i+1}}^{n}<0 \\
0, & v_{p_{i+1}}^{n} \geq 0\end{cases} \\
& v_{p_{T}}^{n}= \begin{cases}v_{p_{i-1}}^{n}, & v_{p_{i-1}}^{n}>0 \\
0, & v_{p_{i+1}}^{n} \leq 0\end{cases}
\end{aligned}
$$

An adjustment is required for calculating $F(I)$ using the continuum framework because light intensity will vary across the grid cell due to light attenuation with depth. To reflect this, the integral of light over the grid cell is used and $F(I)$ becomes: 


$$
F(I)=\frac{e}{\alpha \Delta z}\left[e^{-\gamma_{2}}-e^{-\gamma_{1}}\right]
$$

where

$$
\begin{gathered}
\gamma_{1}=\frac{(1-\beta) I_{0}}{I_{S}} e^{-\alpha(i-1) \Delta z} \\
\gamma_{2}=\frac{(1-\beta) I_{0}}{I_{S}} e^{-\alpha(i) \Delta z}
\end{gathered}
$$

While the above continuum framework accounts for changes in colony density due to the instantaneous growth rate, it does not include the same information about past growth as the particle-tracking framework. To address this, an exponentially-decaying, weighted average of past growth rates in each grid cell was applied to the same continuum framework outlined above (Equation 50).

$$
\rho_{c_{i}}^{n+1}=\frac{\sum_{q=-1}^{Q} \rho_{c_{i}}^{n-q} W^{q}}{\sum_{q=-1}^{Q} W^{q}}
$$

Here, the past densities in the grid cell $i$ are multiplied by a weight $W$ and summed. The total number of timesteps over which to average past densities is given by $Q$. The weight decreases exponentially with time before the present, so that densities predicted at more recent timesteps have greater weights (Equation 51).

$$
W^{q}=e^{-k\left(t^{n+1}-t^{n-q}\right)}
$$

Here, $k$ is the time decay constant for influence of past densities. This is similar to the approach taken by Serizawa et al. (2008), shown here by Equations $15 \& 16$. 
Table 2-3. Dynamic velocity models used in continuum framework

\begin{tabular}{|c|c|}
\hline Model & Equations \\
\hline $\begin{array}{l}\text { Growth } \\
\text { kinetics }\end{array}$ & 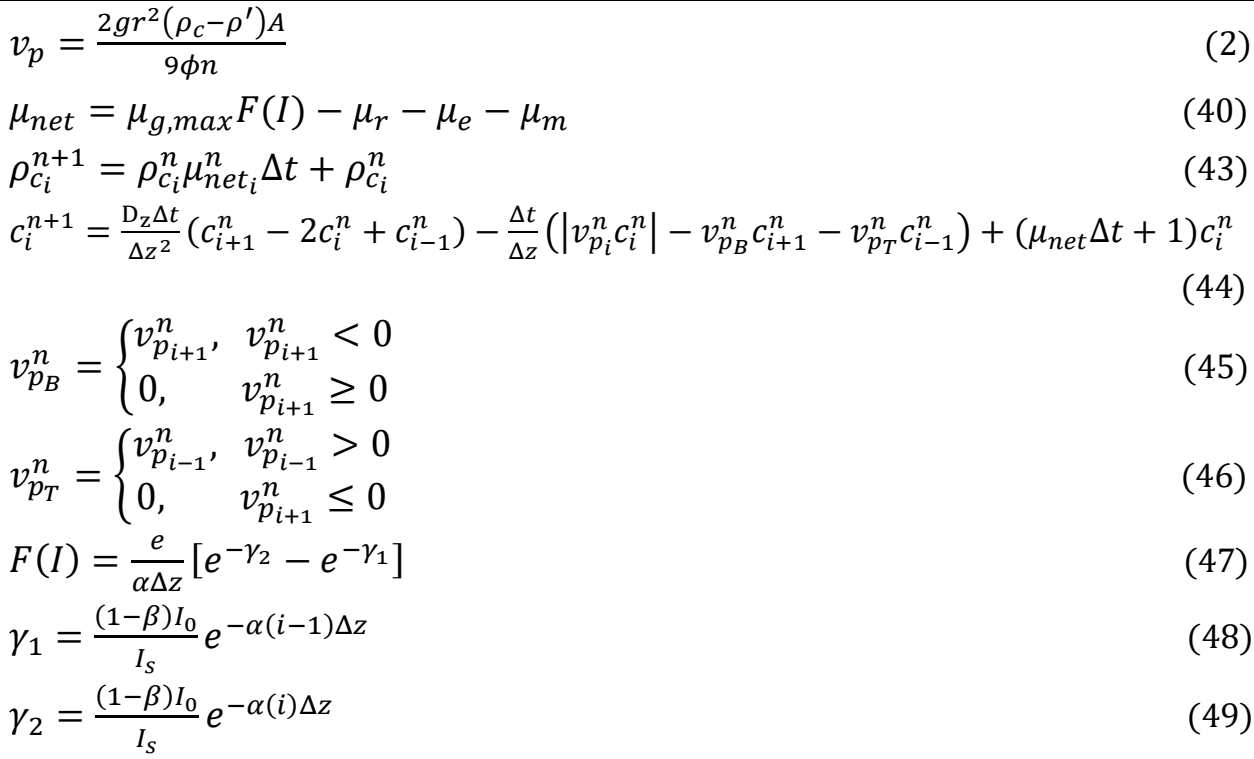 \\
\hline $\begin{array}{l}\text { Growth } \\
\text { kinetics } \\
\text { with time } \\
\text { decay }\end{array}$ & $\begin{array}{l}\rho_{c_{i}}^{n+1}=\frac{\sum_{q=-1}^{Q} \rho_{c_{i}}^{n-q} W^{q}}{\sum_{q=-1}^{Q} W^{q}} \\
W^{q}=e^{-k\left(t^{n+1}-t^{n-q}\right)}\end{array}$ \\
\hline $\begin{array}{l}\text { Visser et al. } \\
\text { (1997) }\end{array}$ & $\begin{array}{l}\rho_{c_{i}}^{n+1}=\left(c_{1} I e^{-I / I_{0}}+c_{2}\right) \Delta t+\rho_{c_{i}}^{n} \\
\rho_{c_{i}}^{n+1}=\left(f_{1}\left(\rho_{c_{i}}^{n}+\rho *_{c_{i}}^{n}\right)+f_{2}\right) \Delta t+\rho_{c_{i}}^{n} \\
I_{i}=\frac{I_{0}(1-\beta)}{-k \Delta z}\left(e^{-\alpha z_{i}}-e^{-\alpha z_{i-1}}\right)\end{array}$ \\
\hline $\begin{array}{l}\text { Light } \\
\text { function }\end{array}$ & $\rho_{c_{i}}^{n+1}=\left(c_{1} F\left(I_{i}^{n}\right)-c_{2}\right) \Delta t+\rho_{c_{i}}^{n}$ \\
\hline $\begin{array}{l}\text { Light } \\
\text { function } \\
\text { with time } \\
\text { decay }\end{array}$ & (2), (43-51), (56) \\
\hline
\end{tabular}


Table 2-4. Dynamic velocity models used in particle-tracking framework

\begin{tabular}{|l|lr|}
\hline \multicolumn{1}{|c|}{ Model } & \multicolumn{1}{|c|}{ Equations } \\
\hline \multirow{5}{*}{ Growth kinetics } & $v_{p}=\frac{2 g r^{2}\left(\rho_{c}-\rho^{\prime}\right) A}{9 \phi n}$ & (2) \\
& $z_{p_{i}}^{n+1}=z_{p_{i}}^{n}+v_{p_{i}}^{n} \Delta t+R \sqrt{6 D_{z_{i}}^{n} \Delta t}$ & $(35)$ \\
& $\mu_{n e t}=\mu_{g, \max } F(I)-\mu_{r}-\mu_{e}-\mu_{m}$ & $(40)$ \\
& $F(I)=\frac{I}{I_{s}} e^{-\frac{I}{I_{s}}+1}$ & $(41)$ \\
& $\rho_{c_{i}}^{n+1}=\rho_{c_{i}}^{n} \mu_{n e t_{i}}^{n} \Delta t+\rho_{c_{i}}^{n}$ & (2), (35) \\
\hline Visser et al. (1997) & $I(z)=(1-\beta) I_{0} e^{-\alpha z}$ & (52) \\
& $\rho_{c_{i}}^{n+1}=\left(c_{1} I e^{-I / I_{0}}+c_{2}\right) \Delta t+\rho_{c_{i}}^{n}$ & (53) \\
\hline Light function & $\rho_{c_{i}}^{n+1}=\left(f_{1}\left(\rho_{c_{i}}^{n}+\rho_{*}\right)+f_{2}\right) \Delta t+\rho_{c_{i}}^{n}$ & (35), (41) \\
\hline
\end{tabular}

\section{Visser et al. (1997) Model}

The equations from Visser et al. (1997), Equations $10 \& 11$, were also applied to both the continuum and particle-tracking frameworks. Equations 52 and 53 give the numerical solutions to those equations. A correction factor, $\rho_{*}$, was applied to Equation 11 to reflect the difference between the buoyant density modeled here and the non-buoyant density on which the equations in that study were based (Equation 53).

$$
\begin{gathered}
I_{i}^{n} \geq I_{c}, \rho_{c_{i}}^{n+1}=\left(c_{1} I e^{-I / I_{0}}+c_{2}\right) \Delta t+\rho_{c_{i}}^{n} \\
I_{i}^{n}<I_{c}, \rho_{c_{i}}^{n+1}=\left(f_{1}\left(\rho_{i_{i}}^{n}+\rho_{*}\right)+f_{2}\right) \Delta t+\rho_{c_{i}}^{n}
\end{gathered}
$$

It was assumed that $\rho_{i}$ was the last density experienced by a particle or grid cell while the irradiance was greater than $I_{c}$, the compensation irradiance. For example, 
at dawn $\rho_{i}$ for a particle or grid cell is the density of that particle or grid cell during the last timestep at the end of the previous day during which it experienced an irradiance greater than $I_{c}$. If a particle moves to a location where the light intensity is less than $I_{c}$, or if the light intensity at a particle's location or in a grid cell becomes less than $I_{c}$ over time, $\rho_{i}$ is the last density of that particle or grid cell before light intensity changed. In the continuum framework, light intensity was averaged across a grid cell depth using Equation 54.

$$
I_{i}=\frac{I_{0}(1-\beta)}{-k \Delta z}\left(e^{-\alpha z_{i}}-e^{-\alpha z_{i-1}}\right)
$$

The parameter values used in Visser et al. (1997) were converted from units of $\mu$ mol photon $s^{-1}$ to Watts to align with the units used for solar irradiance in CEQUAL-W2 (Table 2-5). A conversion factor of $2 \mu \mathrm{mol}$ photon $s^{-1} /$ Watt was used, based on a review of conversion factors by Jacovides et al. (2004).

Table 2-5 Converted parameter values from Visser et al. (1997)

\begin{tabular}{|l|c|c|c|c|c|}
\hline Parameter & $\begin{array}{c}I_{c}, \\
W m^{-2}\end{array}$ & $\begin{array}{c}c_{1}, \\
s^{2} m^{-3}\end{array}$ & $\begin{array}{c}c_{2}, \\
k g ~ m^{-3} s^{-2}\end{array}$ & $\begin{array}{c}f_{1}, \\
s^{-1}\end{array}$ & $\begin{array}{c}f_{2}, \\
\mathrm{~kg} \mathrm{~m}^{-3} \mathrm{~s}^{-1}\end{array}$ \\
\hline Converted Value & 5.45 & $5.333 \times 10^{-5}$ & $-2.75 \times 10^{-4}$ & $-1.587 \times 10^{-5}$ & 0.0164 \\
\hline
\end{tabular}

\section{Light Function Model}

A third approach to modeling light-dependent density change used here was based on the above two approaches, as well as the model of Kromkamp and Walsby (1990). In both Visser et al. (1997) and Kromkamp and Walsby (1990), density increase was modeled using equations similar to growth kinetic equations with additional calibration coefficients. In Kromkamp and Walsby, (1990), a Monod type 
equation was used to model density increase with light and a linear relationship was used to model density decrease in the dark (Equation 1). In Visser et al. (1997), density increase was modeled using an exponential term which accounts for photoinhibition and density decrease was modeled with a linear term. In the light function buoyancy model described here, density change was assumed to follow the same response to light as growth kinetics, including photoinhibition. However, calibration coefficients $c_{1}$ and $c_{2}$ are used rather than the growth rates described above, and growth is assumed to be zero-order rather than first-order (Equation $55)$.

$$
\frac{\partial \rho}{\partial t}=c_{1} F(I)-c_{2}
$$

This allows density change to be calculated separately from population growth while still representing the relationship between density change and light. In the absence of light, density decreases at a constant rate. The numerical solution for Equation 55 is given by Equation 56.

$$
\rho_{c_{i}}^{n+1}=\left(c_{1} F\left(I_{i}^{n}\right)-c_{2}\right) \Delta t+\rho_{c_{i}}^{n}
$$

In all density-change approaches, colonies were assumed to have a minimum and maximum allowable density $\left(\rho_{c_{\min }}\right.$ and $\rho_{c_{\max }}$, respectively) as well as a constant radius $\left(r_{c}\right)$ based on values found in field studies (Table 2-6). When predicted densities were greater than the maximum or less than the minimum allowed values, the value was set to $\rho_{c_{\max }}$ or $\rho_{c_{\min }}$, respectively. It was also necessary to define 
initial densities $\left(\rho_{0}\right)$ for all colonies (particle-tracking) or colonies within a grid cell (continuum).

Table 2-6 Literature values for biological parameters of cyanobacteria used in models

\begin{tabular}{|c|c|c|c|c|c|c|}
\hline Study & Identifier & $\begin{array}{c}\text { Minimum } \\
\text { density, } \\
k g m^{-3}\end{array}$ & $\begin{array}{c}\text { Maximum } \\
\text { density, } \\
\text { kg } \boldsymbol{m}^{-3}\end{array}$ & $\begin{array}{c}\text { Colony } \\
\text { radius, } \\
\boldsymbol{\mu m}\end{array}$ & $\begin{array}{c}\text { Saturating } \\
\text { light } \\
\text { intensity, } \\
W m^{-2}\end{array}$ & $\begin{array}{l}\text { Maximum } \\
\text { growth } \\
\text { rate, } \\
\text { day }^{-1}\end{array}$ \\
\hline $\begin{array}{l}\text { Reynolds } \\
\text { (1984) }\end{array}$ & - & - & - & $25-1000$ & - & - \\
\hline \multirow{4}{*}{$\begin{array}{l}\text { Reynolds et al. } \\
\text { (1987) }\end{array}$} & cyanobacteria & - & - & - & - & $0.6-0.8$ \\
\hline & M. aeruginosa & 985 & 1005 & $120-3200$ & - & - \\
\hline & A. flos-aqua & 920 & 1030 & $28-100$ & - & - \\
\hline & $P$, agardhii & 985 & 1085 & $13.7-18.3$ & - & - \\
\hline $\begin{array}{l}\text { Nakamura et al. } \\
\text { (1993) }\end{array}$ & Microcystis sp. & - & - & $10-300$ & - & - \\
\hline $\begin{array}{l}\text { Visser et al. } \\
\text { (1997) }\end{array}$ & Microcystis sp. & - & - & - & 139 & - \\
\hline $\begin{array}{l}\text { Long et al. } \\
\text { (2001) }\end{array}$ & M. aeruginosa & - & - & - & - & 1.2 \\
\hline $\begin{array}{l}\text { Wu and Song } \\
\text { (2008) }\end{array}$ & M. aeruginosa & - & - & - & $119-244$ & - \\
\hline Wu et al. (2009) & M. aeruginosa & - & - & - & $65-119$ & - \\
\hline $\begin{array}{l}\text { Zhang et al. } \\
\text { (2011) }\end{array}$ & M. aeruginosa & - & - & - & $75-392$ & - \\
\hline $\begin{array}{l}\text { Zhu et al. (2014, } \\
\text { 2018) }\end{array}$ & Microcystis sp. & 967 & 997 & $10-350$ & - & - \\
\hline $\begin{array}{l}\text { Rowe et al. } \\
\text { (2016) }\end{array}$ & Microcystis sp. & - & - & $\begin{array}{c}\text { 12.5-370, } \\
\text { median: } \\
58.5\end{array}$ & - & - \\
\hline
\end{tabular}

\subsubsection{Preliminary Results}

Before application to field studies of cyanobacteria, all models were first tested with generic inputs to demonstrate their ability to predict a diurnal migration pattern. In these preliminary models, population growth was ignored in order to focus on vertical movement. This is equivalent to removing the source-sink term 
from Equation 32. For preliminary models, it was assumed that $I_{0}$ followed a halfsine function (Equation 57).

$$
I_{0}=\frac{I_{\max }}{2}\left[\sin \left(\frac{2 \pi t}{86,400 s}+\frac{3 \pi}{2}\right)+\left|\sin \left(\frac{2 \pi t}{86,400 s}+\frac{3 \pi}{2}\right)\right|\right]
$$

This assumes 12 hours of daylight and that $t=0$ corresponds to midnight. The amplitude $\left(I_{\max }\right)$ is the maximum daily solar irradiance at the surface of the water and is assumed to occur at noon.

In preliminary predefined velocity models in the particle-tracking framework, a designated number of particles was used and each was assigned the same mass. This mass was determined based on the initial concentration used in the continuum framework $\left(c_{0}\right)$ and the total number of particles $P$ (Equation 58).

$$
m=\frac{c_{0} \Delta z B x}{P}
$$

In dynamic velocity models, particles were assumed to have an initial density and constant radius. In these models, the number of particles was determined based on these variables and the initial concentration used in continuum framework models (Equation 59).

$$
P=\frac{\mathrm{c}_{0} \Delta z B x}{\mathrm{~K} \rho_{c_{0}} \frac{4}{3} \pi r_{p}^{3}}
$$

Here, $\mathrm{K}$ is the number of cyanobacteria colonies represented by one model particle. This was included to reduce the number of particles necessary based on initial density and radius values. 
The same general model setup was used for all preliminary models (Table 2-7). Some parameter values differed between models due to differences in the models (Table 2-8, Table 2-9, Table 2-10, \& Table 2-11). In all models, concentration (or number of particles) was initially uniformly distributed over the depth of the model grid.

Table 2-7 Values used in setup of preliminary models

\begin{tabular}{|c|c|c|}
\hline Variable & Description & Value \\
\hline$H, m$ & Height of water column & 10 \\
\hline$x, m$ & Length of control volume & 1 \\
\hline$B, m$ & Width of control volume & 1 \\
\hline$\Delta z, m$ & Grid cell height & 0.5 \\
\hline$\Delta t, s$ & Model timestep & 60 \\
\hline$D_{z}, m^{2} s^{-1}$ & Diffusion coefficient & $10^{-5}$ \\
\hline$c_{0}, m g l^{-1}$ & Initial concentration & 0.01 \\
\hline$I_{0, \text { max }}, W m^{-2}$ & Maximum solar irradiance & 1000 \\
\hline $\mathrm{K}$ & Number of cyanobacteria colonies represented by one particle & 100 \\
\hline
\end{tabular}

The same amplitude and phase were used for both predefined velocity models in continuum and particle-tracking frameworks (Table 2-8). The predefined, time-varying velocity models showed a distinct and symmetrical vertical migration pattern (Figure 2-2). Concentrations were more intense at the surface than at the bed in models using both particle-tracking and continuum frameworks, due to the initial motion being in the upward direction (Figure 2-2 \& Figure 2-3).

Concentrations were greater at the bed in the model using the continuum framework versus the particle-tracking framework (Figure 2-4). This is also due to the boundary condition that allows particles to stay at the surface but forces them to move up if they encounter the bed. 
Table 2-8 Values used in preliminary predefined velocity models

\begin{tabular}{|c|c|c|c|}
\hline Variable & Description & Time-varying velocity & Belov \& Giles (1997) \\
\hline$A, \mathrm{~m}$ & Migration amplitude & 2 & 2 \\
\hline \multirow{2}{*}{, $\mathrm{rad}$} & Phase offset & $\frac{\pi}{2}$ & $\frac{\pi}{2}$ \\
\hline$\alpha, m^{-1}$ & Light attenuation coefficient & - & 0.25 \\
\hline$P$ & Number of particles & 1000 & 1000 \\
\hline
\end{tabular}

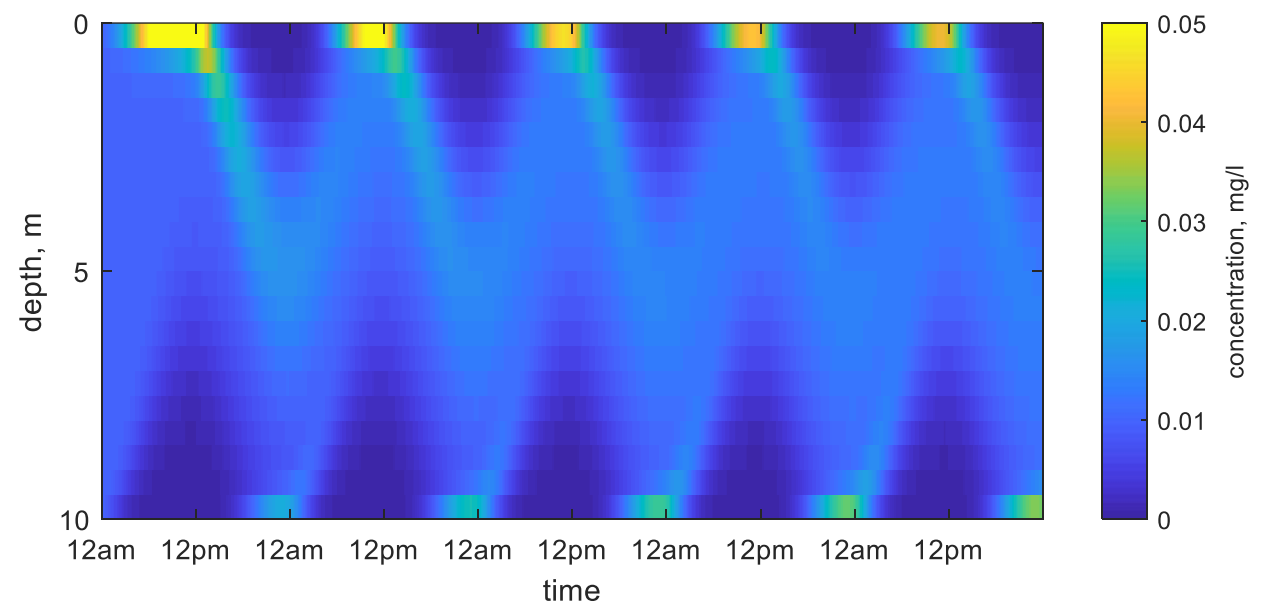

Figure 2-2 Five-day time series of concentration at depth predicted by preliminary time-varying velocity model (continuum)

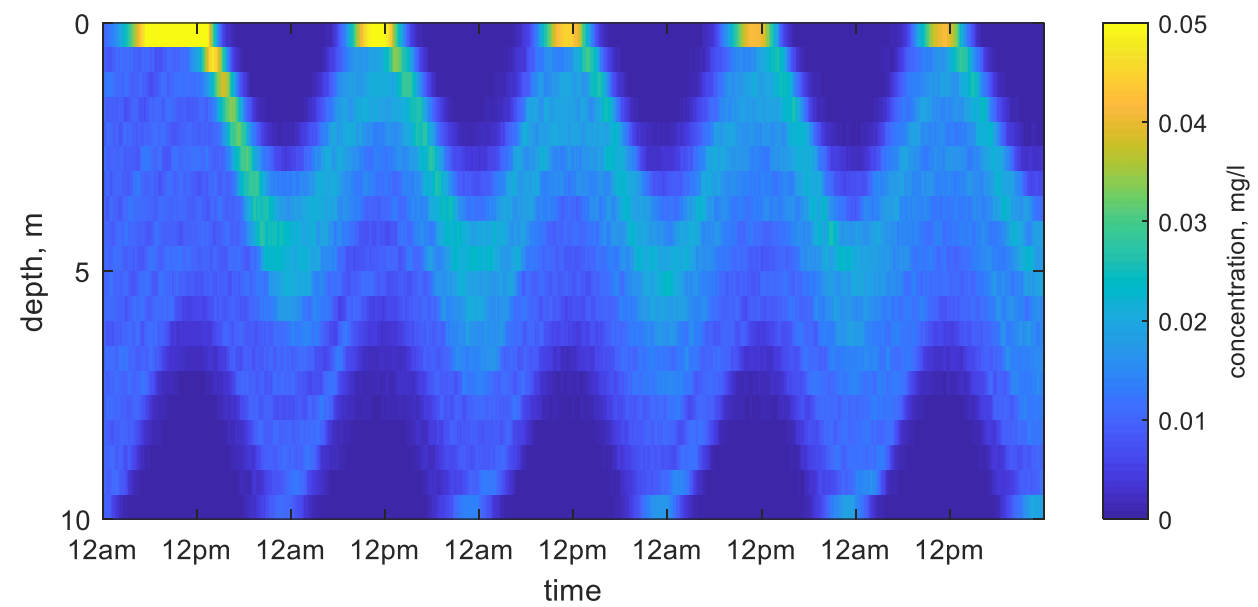

Figure 2-3 Five-day time series of concentration at depth predicted by preliminary time-varying velocity model (particle-tracking) 

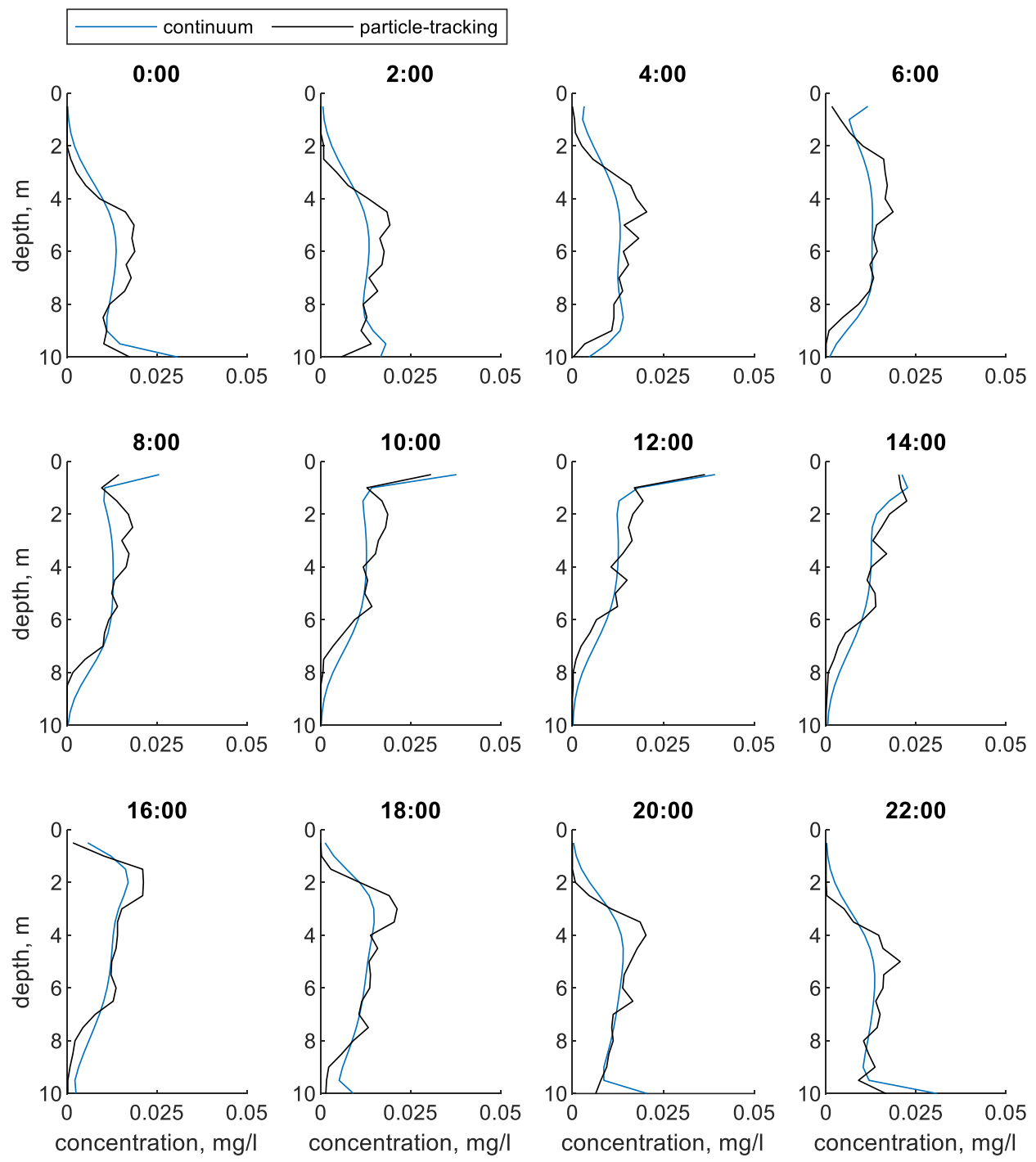

Figure 2-4 Concentration profiles at two-hour intervals predicted by preliminary time-varying velocity models in continuum and particle-tracking frameworks

In the models based on equations from Belov \& Giles (1997), concentration was greater towards the surface than the bed (Figure 2-5 \& Figure 2-6). This is the effect of the exponential term in Equation 38, which decreases velocity near the 
surface. The concentration close to the bed looks similar to that predicted by the time-varying model, as the effect of the exponential term in Equation 38 is diminished near the bed. Models in the continuum and particle-tracking frameworks produced similar results using this approach (Figure 2-7).

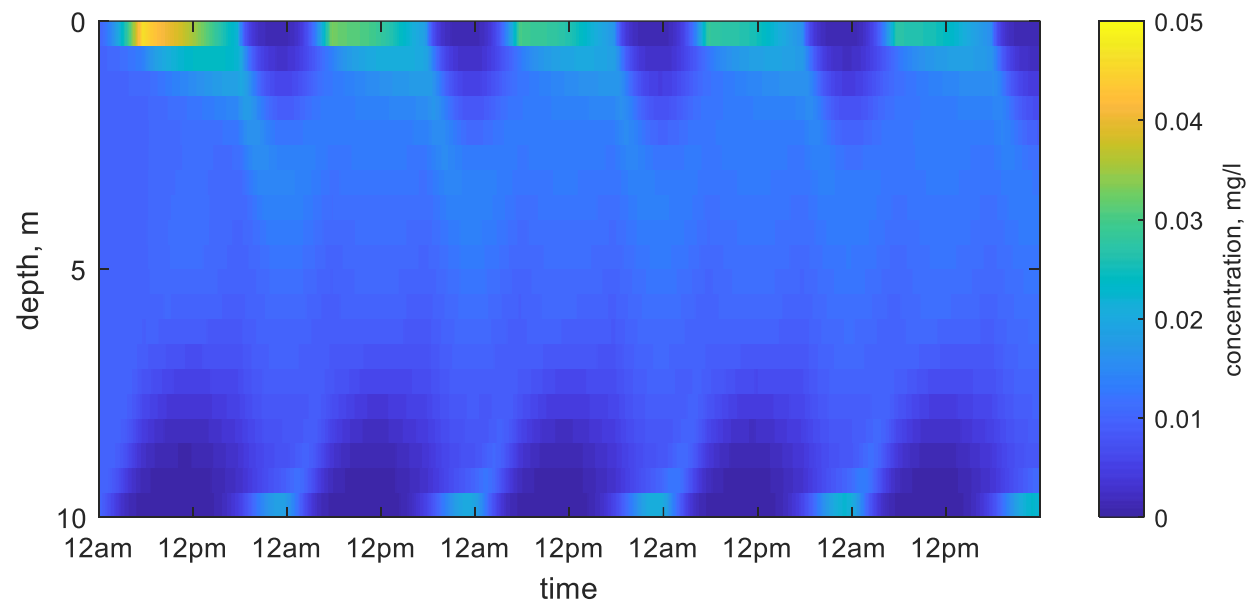

Figure 2-5 Five-day time series of concentration at depth predicted by preliminary Belov \& Giles (1997) model (continuum)

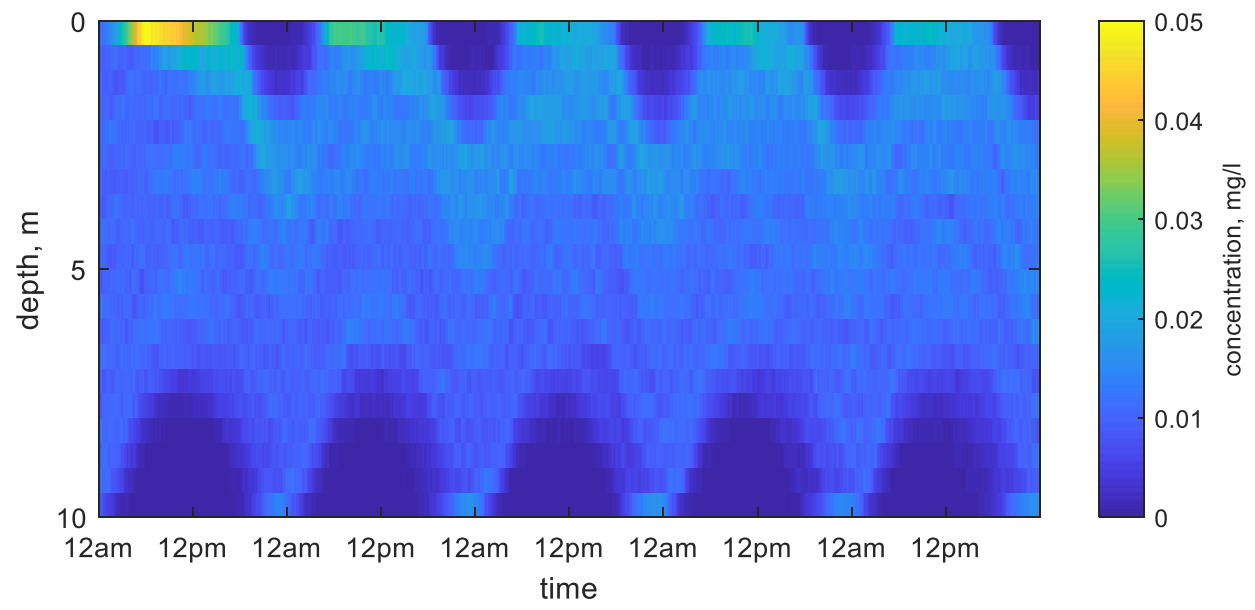

Figure 2-6 Five-day time series of concentration at depth predicted by preliminary Belov \& Giles (1997) model (particle-tracking) 

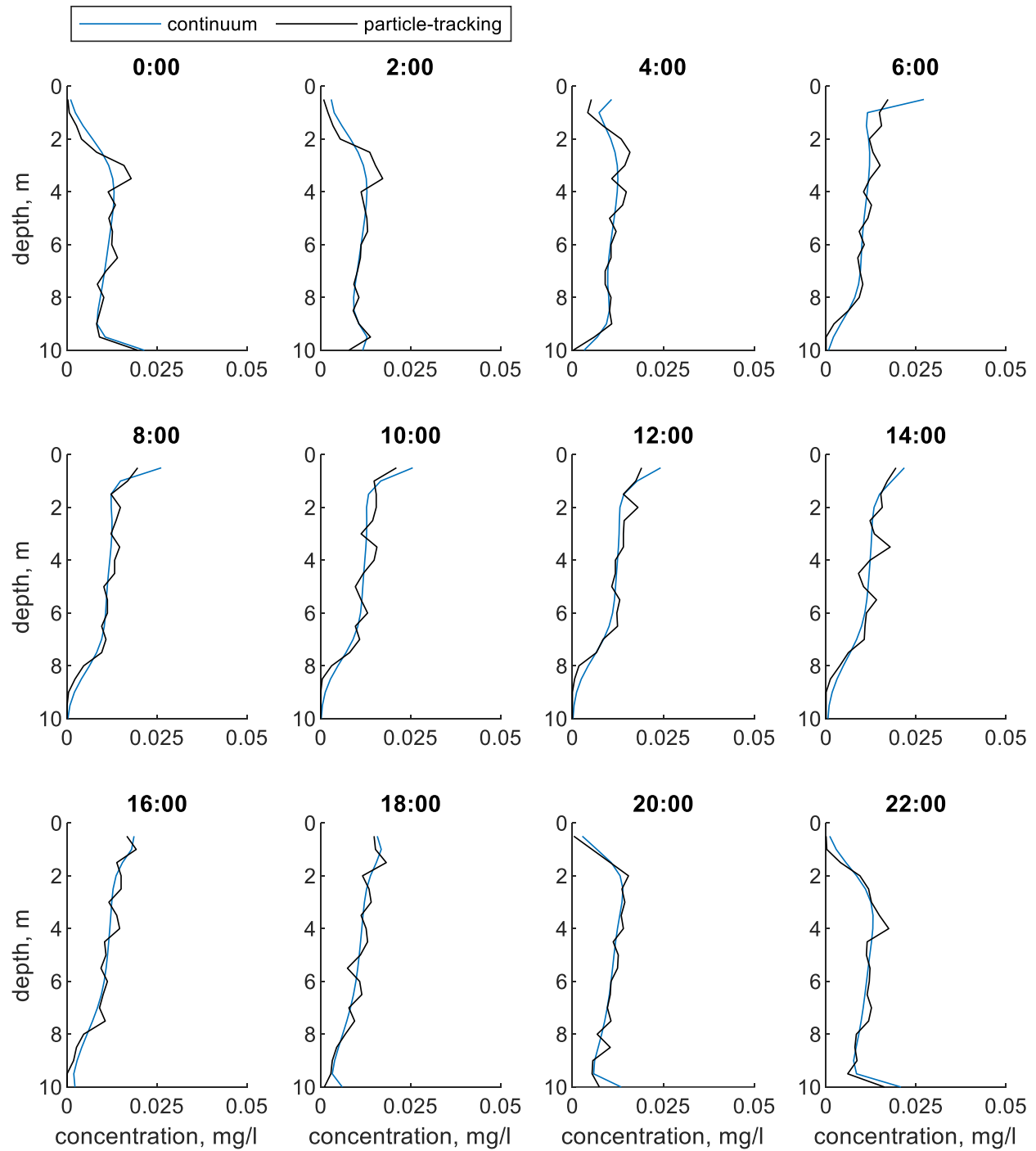

Figure 2-7 Concentration profiles at two-hour intervals predicted by preliminary Belov \& Giles (1997) models in continuum and particle-tracking frameworks

In the dynamic velocity models, values for constants were chosen from a range of typical values found in literature (Table 2-9, Table 2-10, \& Table 2-11). In the growth kinetics and light function models the same constant values were used, while in the model based on Visser et al. (1997), a larger colony radius and smaller minimum colony density were used for better results. 
Table 2-9 Values used in preliminary growth kinetics models

\begin{tabular}{|c|c|c|}
\hline Variable & Description & Value \\
\hline$\alpha, m^{-1}$ & Light attenuation coefficient & 0.5 \\
\hline$\mu_{g, m a x}$, day $^{-1}$ & Maximum growth rate & 0.75 \\
\hline$\mu_{m}$, day $^{-1}$ & Mortality rate & 0.15 \\
\hline$\mu_{e}$, day $^{-1}$ & Excretion rate & 0.04 \\
\hline$\mu_{r}, \mathrm{day}^{-1}$ & Respiration rate & 0.04 \\
\hline$I_{s}, \mathrm{~W} \mathrm{~m}^{-2}$ & Saturating light intensity & 150 \\
\hline$r_{c}, \mu m$ & Colony radius & 50 \\
\hline$\rho_{\min }, \mathrm{kg} \mathrm{m}^{-3}$ & Minimum colony density & 940 \\
\hline$\rho_{\max }, \mathrm{kg} \mathrm{m}^{-3}$ & Maximum colony density & 1065 \\
\hline$\rho_{0}, \mathrm{~kg} \mathrm{~m}^{-3}$ & Initial colony density & 980 \\
\hline$k, \mathrm{day}^{-1}$ & Time decay constant for past densities & 5 \\
\hline
\end{tabular}

In the continuum framework, the growth kinetics model showed a diurnal migration pattern of colonies rising to the surface in the evening, staying there for several hours, and descending in the afternoon (Figure 2-8). The descending limb of the migration curve is steeper than the ascending limb. A similar pattern is seen in the continuum framework with time decay. In the time decay model compared to the model without time decay, colonies reach the surface later in the morning and do not spend as long there before descending (Figure 2-9). The asymmetries of the migration curve are more smoothed out in the time decay model and the time of maximum depth occurs later. 


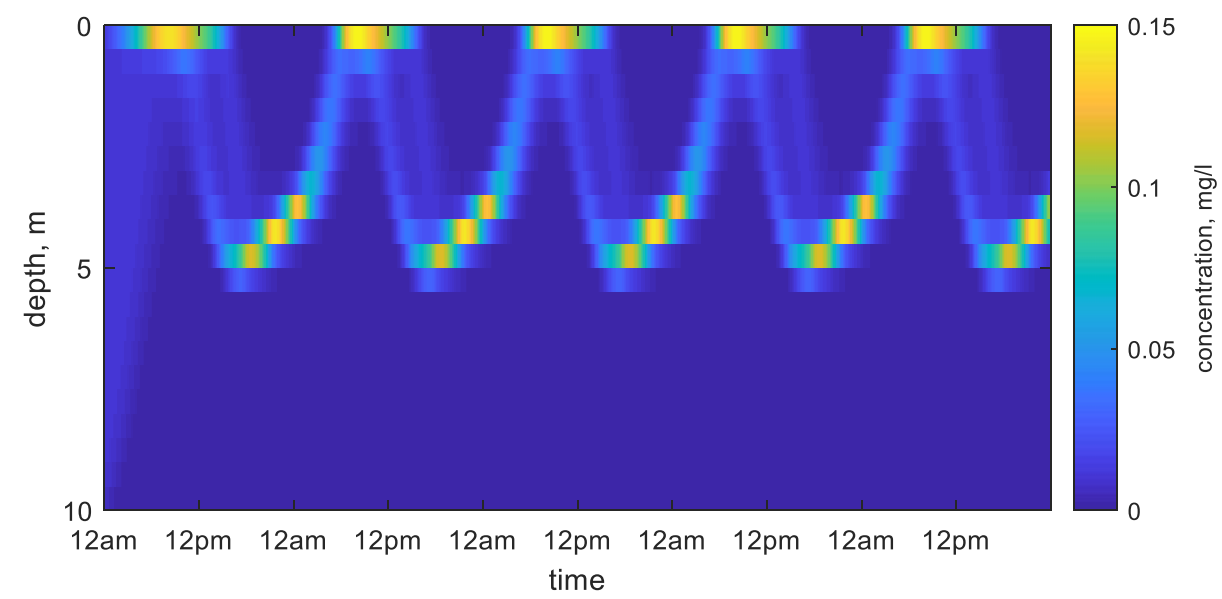

Figure 2-8 Five-day time series of concentration at depth predicted by preliminary growth kinetics model without time decay (continuum)

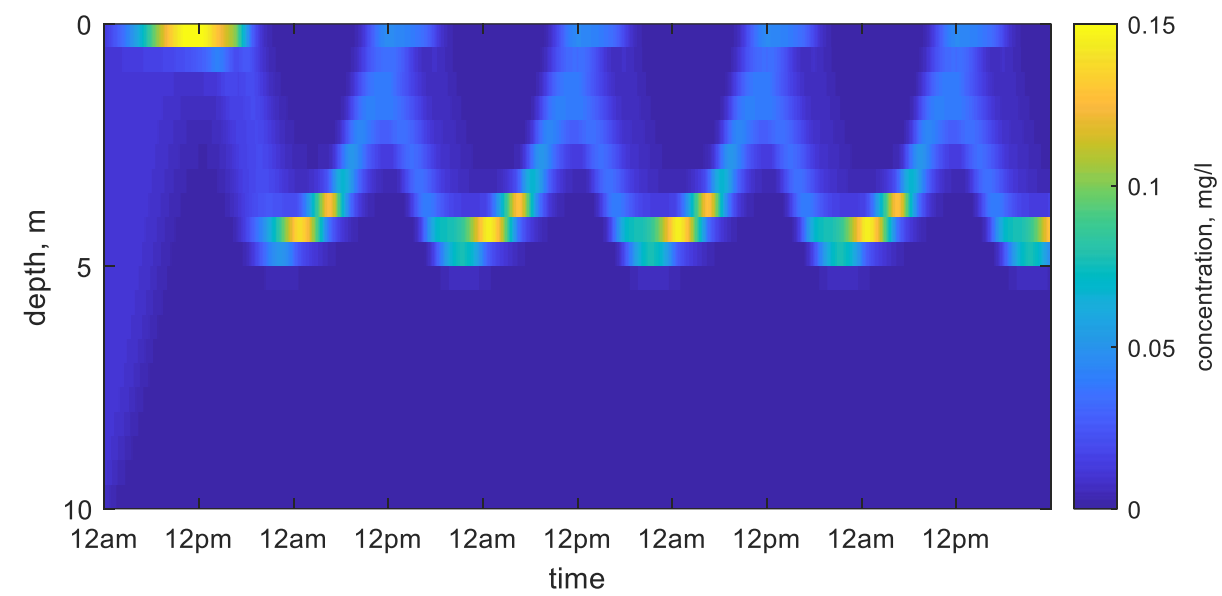

Figure 2-9 Five-day time series of concentration at depth predicted by preliminary growth kinetics model with time decay (continuum)

Results from the particle-tracking framework using the growth kinetics equations show a different pattern than the two continuum-framework models using those equations. A concentration peak at the surface starts earlier in the evening and lasts longer than in the continuum framework (Figure 2-10).

Concentrations are more diffuse below the surface and do not form a strong peak as 
in the continuum framework. However, concentration is higher near the bottom, especially around midnight. Similar patterns can be seen in profile plots of the models, in which the models in the continuum framework show a sharp concentration peak that stays between the middle of the water depth and the surface (Figure 2-11). The model in the particle-tracking framework generally predicts lower concentration, except at the surface where predicted concentration is higher. As in the other dynamic velocity models, the stark difference is due to how density change is calculated in the continuum and particle-tracking frameworks.

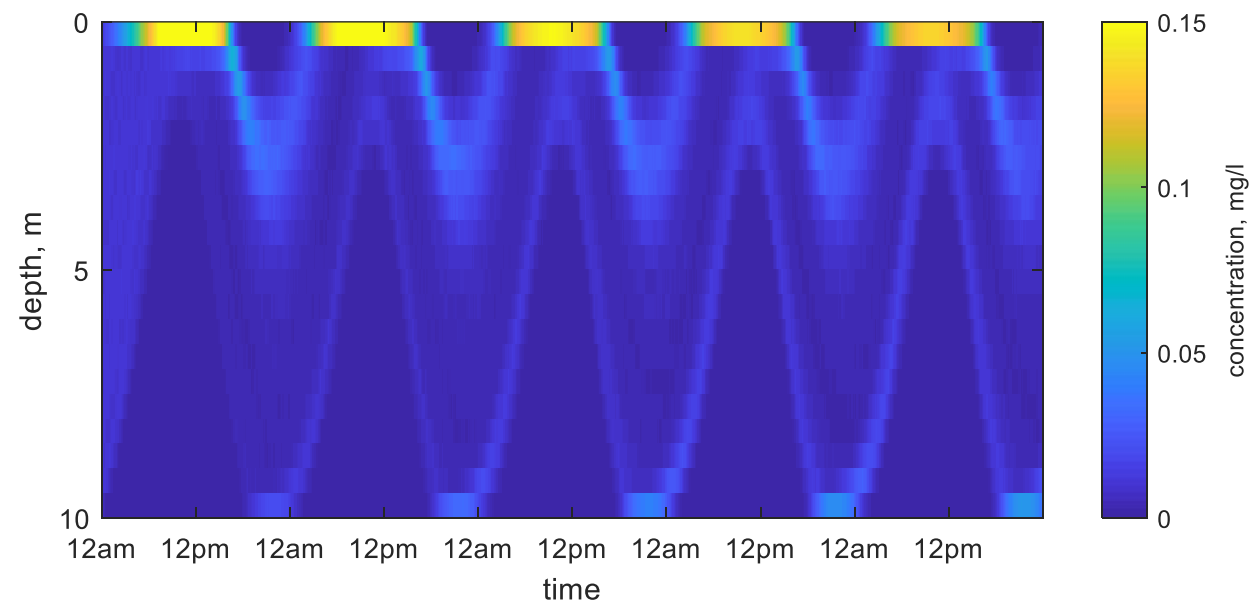

Figure 2-10 Five-day time series of concentration at depth predicted by preliminary growth kinetics model (particle-tracking) 

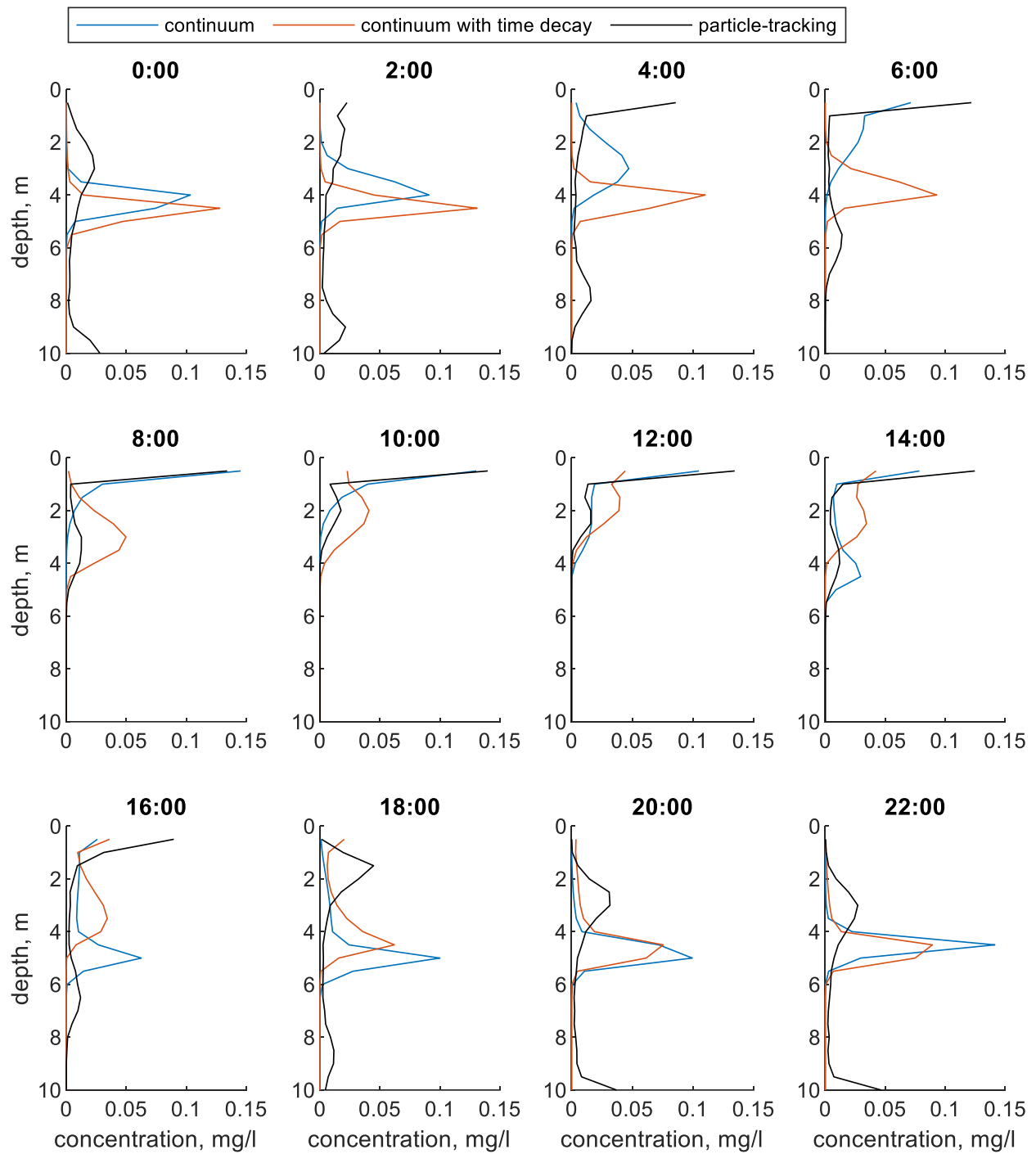

Figure 2-11 Concentration profiles at two-hour intervals predicted by preliminary growth kinetics models (with and without time decay) in continuum and particle-tracking frameworks

In the models based on equations from Visser et al. (1997) values chosen for parameters were similar to those used in the original study (Table 2-10). In that study, a range of colony radii was tested and a higher light attenuation coefficient was used. 
Table 2-10 Values used in preliminary models based on Visser et al. (1997)

\begin{tabular}{|c|c|c|}
\hline Variable & Description & Value \\
\hline$\alpha, \mathrm{m}^{-1}$ & Light attenuation coefficient & 0.5 \\
\hline$I_{s}, \mathrm{~W} \mathrm{~m}^{-2}$ & Saturating light intensity & 150 \\
\hline$r_{c}, \mu \mathrm{m}$ & Colony radius & 125 \\
\hline$\rho_{\text {min }}, \mathrm{kg} \mathrm{m}^{-3}$ & Minimum colony density & 920 \\
\hline$\rho_{\text {max }}, \mathrm{kg} \mathrm{m}^{-3}$ & Maximum colony density & 1065 \\
\hline$\rho_{0}, \mathrm{~kg} \mathrm{~m}^{-3}$ & Initial colony density & 980 \\
\hline$\rho_{*}, \mathrm{~kg} \mathrm{~m}^{-3}$ & Density correction factor & 65 \\
\hline
\end{tabular}

In the continuum framework, concentration profiles did not display the same vertical movement pattern as in other models. Concentrations did not reach the surface, but instead oscillated around five meters deep (Figure 2-12). This is due to the way in which density change and, in turn, velocity, is predicted in the continuum framework. The density in each model grid cell is based on the light received by that grid cell. Grid cells further down receive less light, which causes their predicted densities to be low and their predicted velocities to be in the upward direction. The effect is the opposite in grid cell near the surface that receive more light: predicted densities are high and predicted velocities are downward. This same effect can be seen in the other dynamic velocity models in the continuum framework, with variation in overall pattern due to the different model equations. 


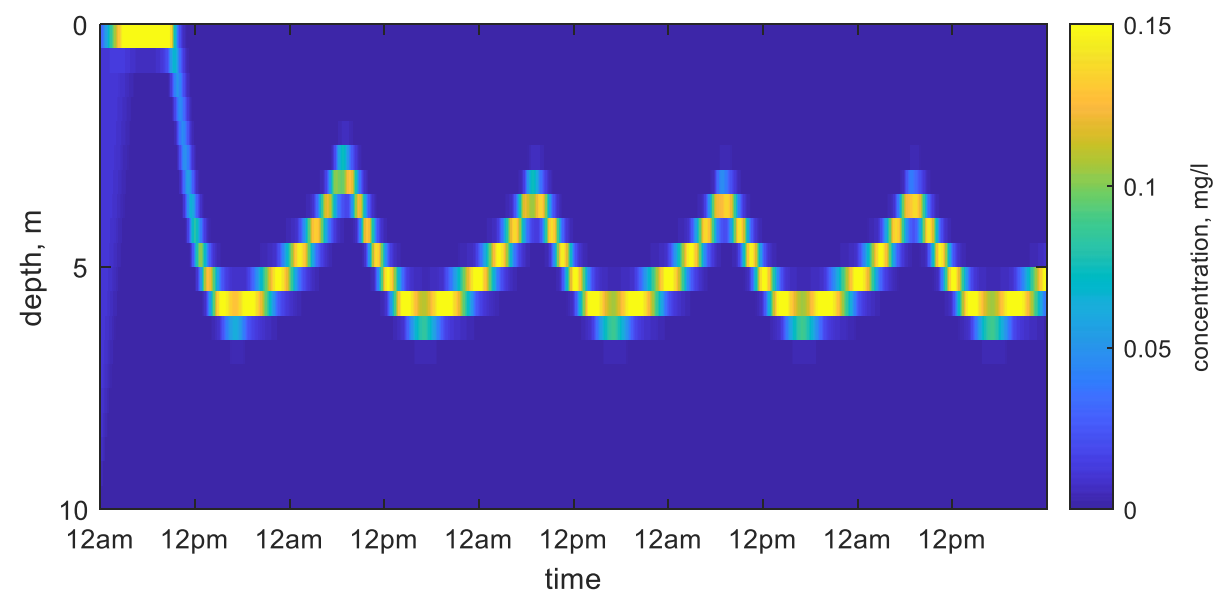

Figure 2-12 Five-day time series of concentration at depth predicted by preliminary Visser et al. (!997) model (continuum)

In the particle-tracking framework, the Visser et al. (1997) equations predicted a more extensive migration path. Strong concentration peaks form at the bed and surface, while in between a distinct line can be seen along which particles are migrating (Figure 2-13 ). As in the growth kinetics equations, the descending limb of the migration curve is steeper than the ascending limb. High concentration values at the surface are not as prolonged as in the growth kinetics particle model, but bottom concentrations are higher and last longer. Comparison of the continuum and particle-tracking profile plots shows similar behavior to the growth kinetics model. The concentration predicted by the model in the continuum framework stays between the mid-depth and surface, while in the particle-tracking framework it varies from uniformly distributed over depth to high values at the bed and surface (Figure 2-14). 


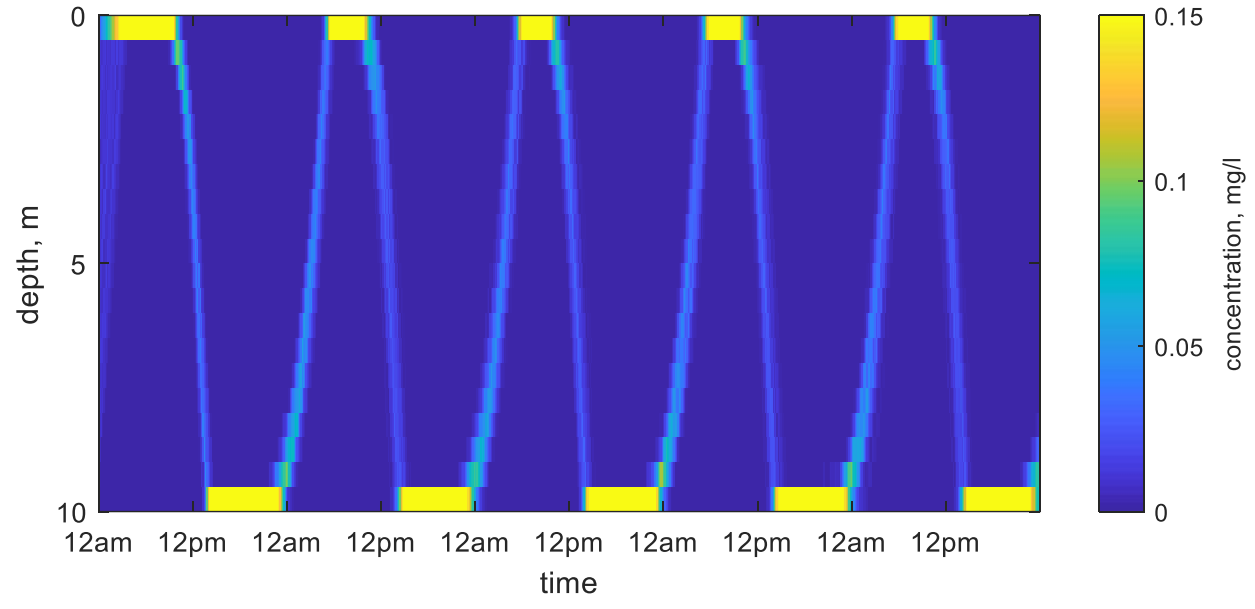

Figure 2-13 Five-day time series of concentration at depth predicted by preliminary Visser et al. (!997) model (particle-tracking) 

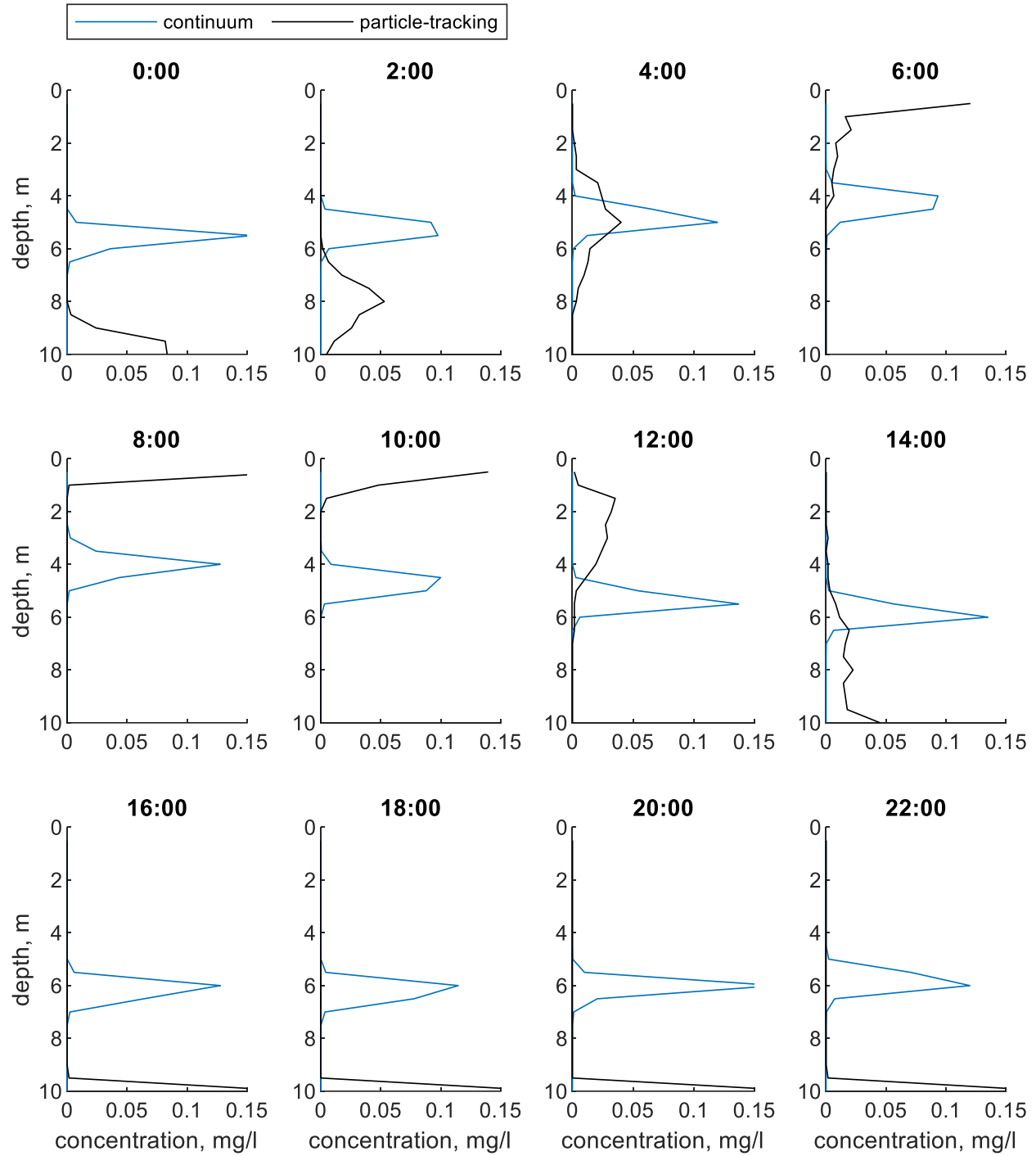

Figure 2-14 Concentration profiles at two-hour intervals predicted by preliminary Visser et al. (1997) models in continuum and particle-tracking frameworks

The light function model shows similar behavior to the growth kinetics model. While the two use similar equations and the same predictor variable, in the light function model density change is determined using a zero-order growth equation rather than a first-order growth equation as in the growth rate model. 
Table 2-11 Values used in preliminary light function models

\begin{tabular}{|c|c|c|}
\hline Variable & Description & Value \\
\hline$\alpha, \mathrm{m}^{-1}$ & Light attenuation coefficient & 0.5 \\
\hline$I_{s}, \mathrm{~W} \mathrm{~m}^{-2}$ & Saturating light intensity & 150 \\
\hline$c_{1}$, day $^{-1}$ & Coefficient of density increase & 0.012 \\
\hline$c_{2}$, day $^{-1}$ & Coefficient of density decrease & 0.0045 \\
\hline$r_{c}, \mu m$ & Colony radius & 50 \\
\hline$\rho_{\min }, \mathrm{kg} \mathrm{m}^{-3}$ & Minimum colony density & 940 \\
\hline$\rho_{\max }, \mathrm{kg} \mathrm{m}^{-3}$ & Maximum colony density & 1065 \\
\hline$\rho_{0}, \mathrm{~kg} \mathrm{~m}^{-3}$ & Initial colony density & 980 \\
\hline$k, \mathrm{day}^{-1}$ & Time decay constant for past densities & 5 \\
\hline
\end{tabular}

The main difference between the concentration predictions from the light function model is that high concentrations stay on the surface for longer than in the growth kinetics model. There is also slightly less diffusion on the descending limb of the migration curve (Figure 2-15).

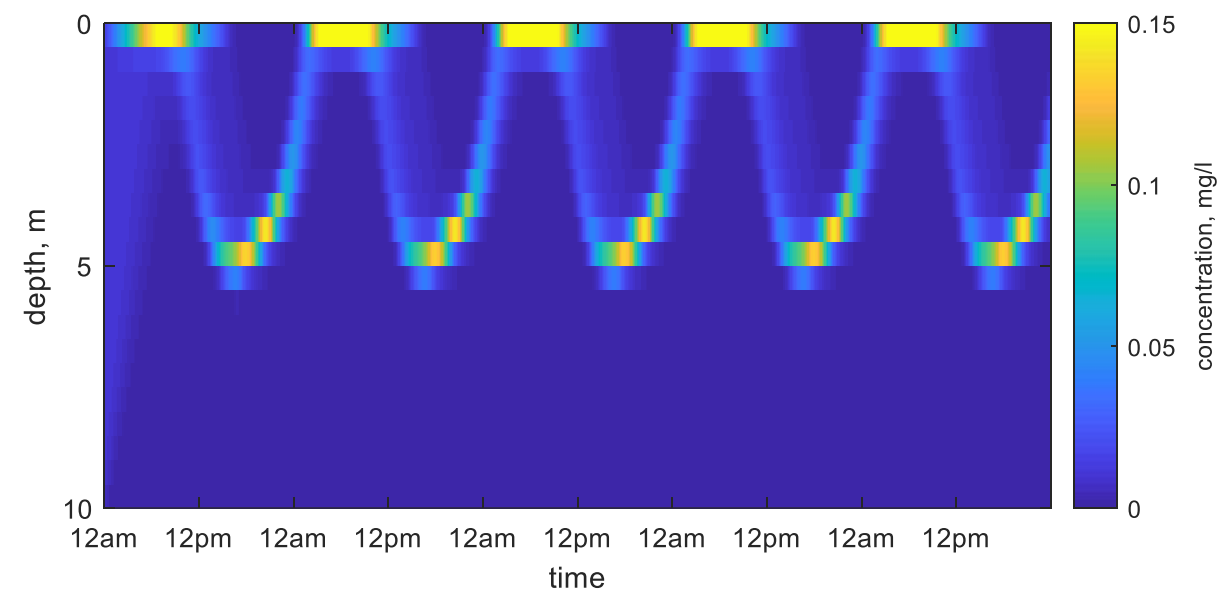

Figure 2-15 Five-day time series of concentration at depth predicted by preliminary light function model without time decay (continuum)

As in the growth kinetics model with time decay, the light function model with time decay shows smoother concentration gradients in time (Figure 2-16). 
Here, as in the light function model without time decay, high concentrations stay at the surface longer than in the corresponding growth kinetics model.

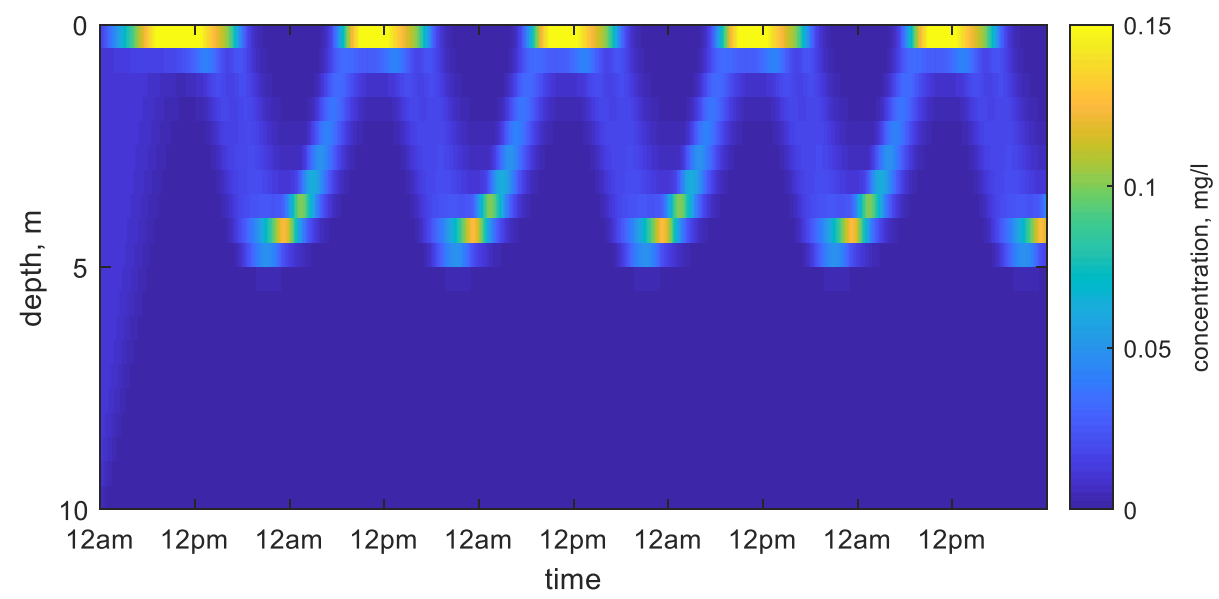

Figure 2-16 Five-day time series of concentration at depth predicted by preliminary light function model with time decay (continuum)

The light function model also showed similar patterns to the growth kinetics model when applied using the particle-tracking framework. Concentration is high at the surface for several hours, then diffuse through the depth of the water column in the evening hours (Figure 2-17). The model using the particle-tracking framework here shows the same linear descent and ascent as in the growth kinetics model using the particle-tracking framework.

In profile plots, the models in the continuum framework with and without time decay are generally in agreement, except in the early morning and late afternoon. The model without time decay in the continuum framework predicts an earlier rise to the surface and an earlier descent to mid-depth (Figure 2-18). 


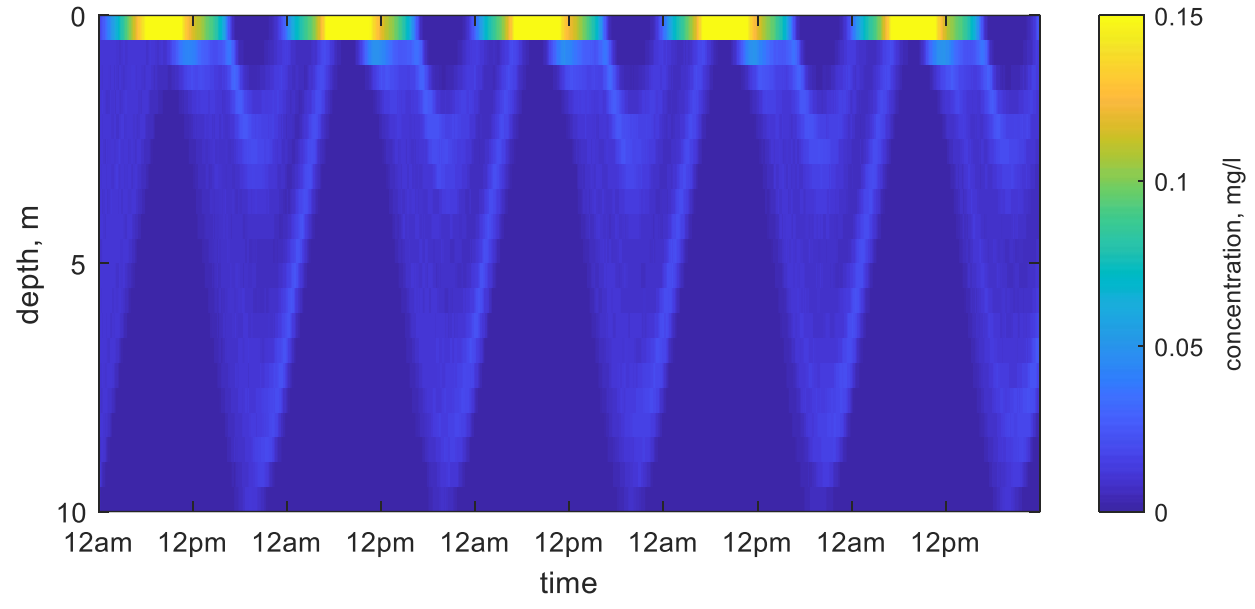

Figure 2-17 Five-day time series of concentration at depth predicted by preliminary light function model (particle-tracking) 

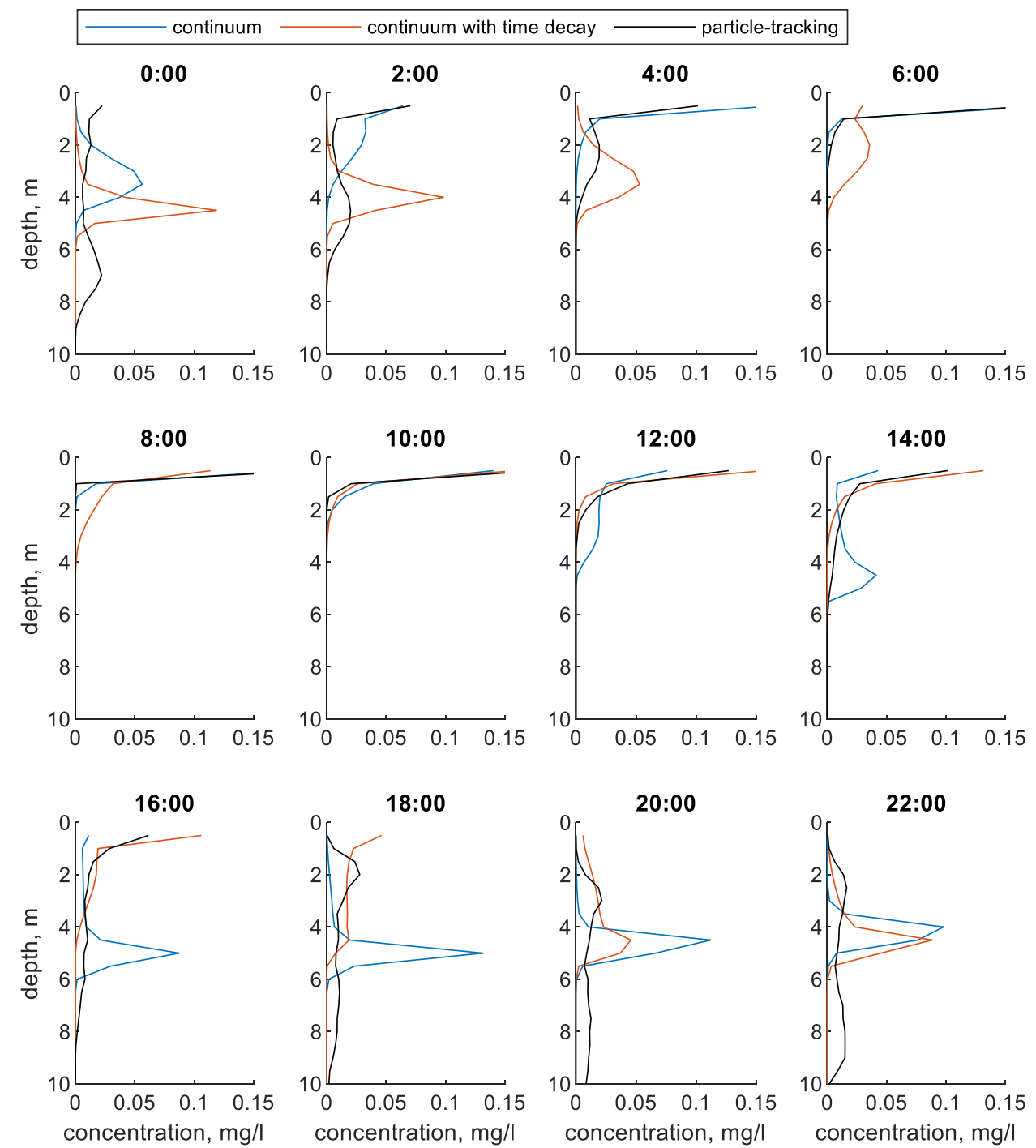

Figure 2-18 Concentration profiles at two-hour intervals predicted by preliminary light function models (with and without time decay) in continuum and particle-tracking frameworks

\subsection{Zooplankton}

\subsubsection{Preliminary Models}

Three different zooplankton models were tested in the continuum and particle-tracking frameworks. All three models solve directly for zooplankton velocity, which is then substituted into Equations 33 \& 34 (continuum) or Equation 
35 (particle-tracking). Where the light at depth in required to solve for velocity, Equations 42 and 54 are used for particle-tracking and continuum frameworks, respectively.

The first model tested was the migration amplitude model from Dodson (1990). This is similar to the time-varying predefined velocity approach used for cyanobacteria, since it uses a sinusoidal velocity function. Here, the amplitude is determined using Equation 17 and the Secchi depth of the water column. To convert between light attenuation coefficient, $\alpha$, and Secchi depth, $D_{S}$, Equation 60 is used.

$$
D_{s}=\frac{1.8}{\alpha}
$$

The amplitude resulting from Equation 17 is used in Equation 37 to solve for velocity.

The model of Andersen and Nival (1991) was also tested, which models krill migration based on change in absolute light intensity at the water surface and light intensity at depth. In this model, Equations 18-23 are solved to obtain a zooplankton velocity. The food-dependent velocity from that model was ignored here in order to focus on light-dependent motion.

The last model of zooplankton migration tested was the model presented in Richards et al. (1996). This model is similar to that of Andersen and Nival (1991), but includes the effect of relative rate of irradiance change. It also includes 
conditions for motion near the bed and surface. For this model, Equations 25-29 are solved to obtain a zooplankton velocity.

As in the cyanobacteria models, light was assumed to follow Equation 57 with a 12-hour photoperiod and $t=0$ corresponding to midnight. Concentration of zooplankton was initially uniform over the depth of the water column for both continuum and particle-tracking frameworks. The grid cell height was the same for each model, while the total height of the water column varied (Table 2-12). This is because the different models predicted different behavior and the same water column height did not adequately show results from all three.

Table 2-12 Values used in preliminary models of zooplankton migration

\begin{tabular}{|c|c|c|c|c|}
\hline Variable & Description & $\begin{array}{c}\text { Dodson } \\
\text { (1990) }\end{array}$ & $\begin{array}{c}\text { Andersen \& Nival } \\
\text { (1991) }\end{array}$ & $\begin{array}{c}\text { Richards et al. } \\
\text { (1996) }\end{array}$ \\
\hline$H, m$ & Height of water column & 25 & 30 & 15 \\
\hline$x, m$ & Length of control volume & 1 & 1 & 1 \\
\hline$B, m$ & Width of control volume & 1 & 1 & 1 \\
\hline$\Delta z, m$ & Grid cell height & 0.5 & 0.5 & 0.5 \\
\hline$\Delta t, s$ & Model timestep & 60 & 15 & 60 \\
\hline$\alpha, m^{-1}$ & Light attenuation coefficient & 0.35 & 0.35 & 0.35 \\
\hline$D_{z}, m^{2} s^{-1}$ & Diffusion coefficient & $10^{\wedge-5}$ & $10^{\wedge-5}$ & $10^{\wedge-5}$ \\
\hline$c_{0}, m g l^{-1}$ & Initial concentration & $10^{-4}$ & $10^{-4}$ & $10^{-4}$ \\
\hline$P$ & Number of particles & 1000 & 1000 & 10000 \\
\hline
\end{tabular}

\subsubsection{Preliminary Results}

Results from the Dodson (1990) model show a regular, symmetric sinusoidal migration pattern similar to that seen in the time-varying velocity model of cyanobacteria (Figure 2-19). Although a constant amplitude was used here, the amplitude could be varied dynamically with time-varying data of light attenuation coefficient or Secchi depth. Results from models in the continuum and particle- 
tracking frameworks are similar, however the model in the continuum framework shows more diffusion than in the particle-tracking framework (Figure 2-20 \& Figure 2-21).

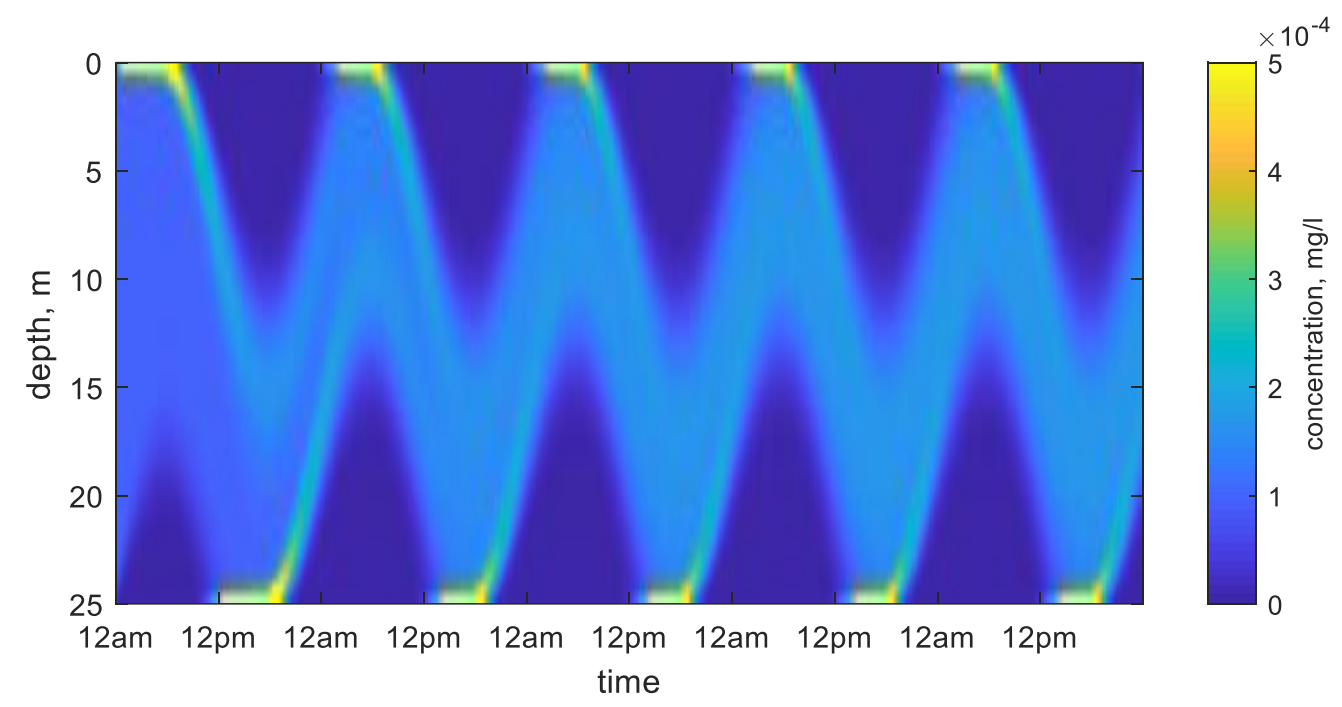

Figure 2-19 Five-day time series of concentration at depth predicted by preliminary Dodson (1990) model (continuum)

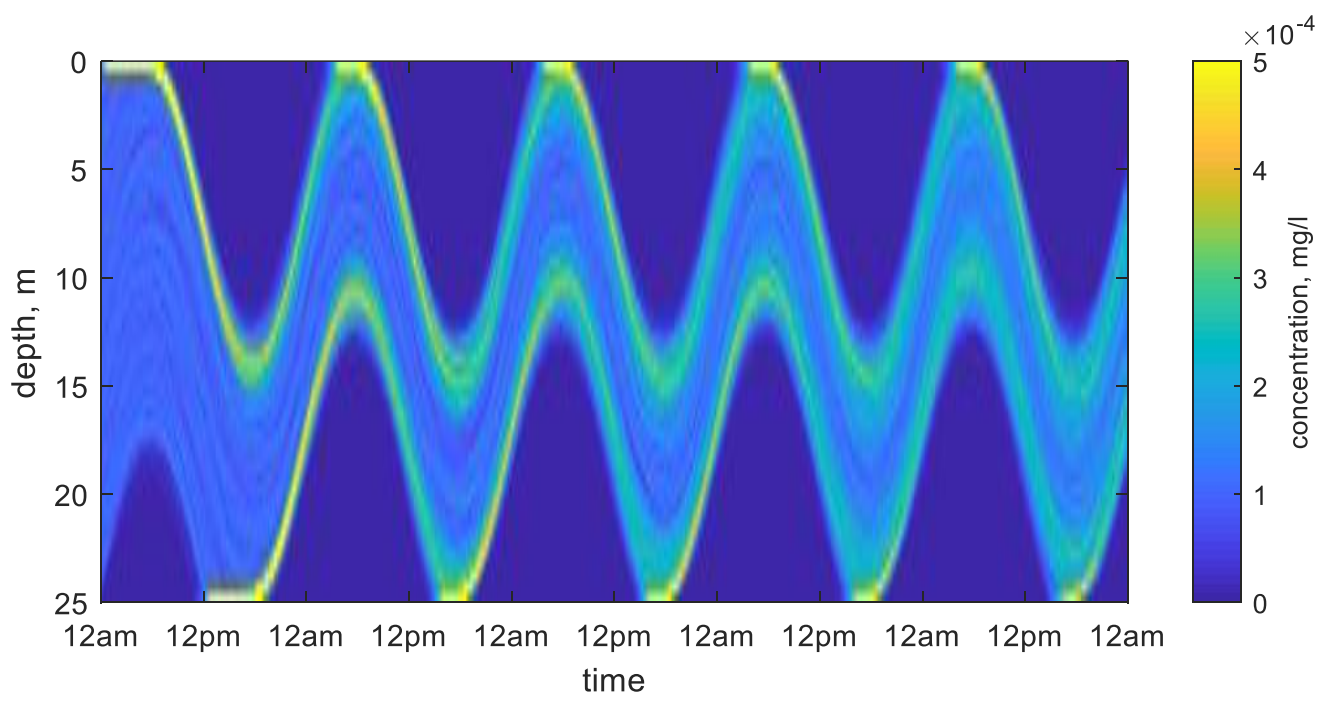

Figure 2-20 Five-day time series of concentration at depth predicted by preliminary Dodson (1990) model (particle-tracking) 

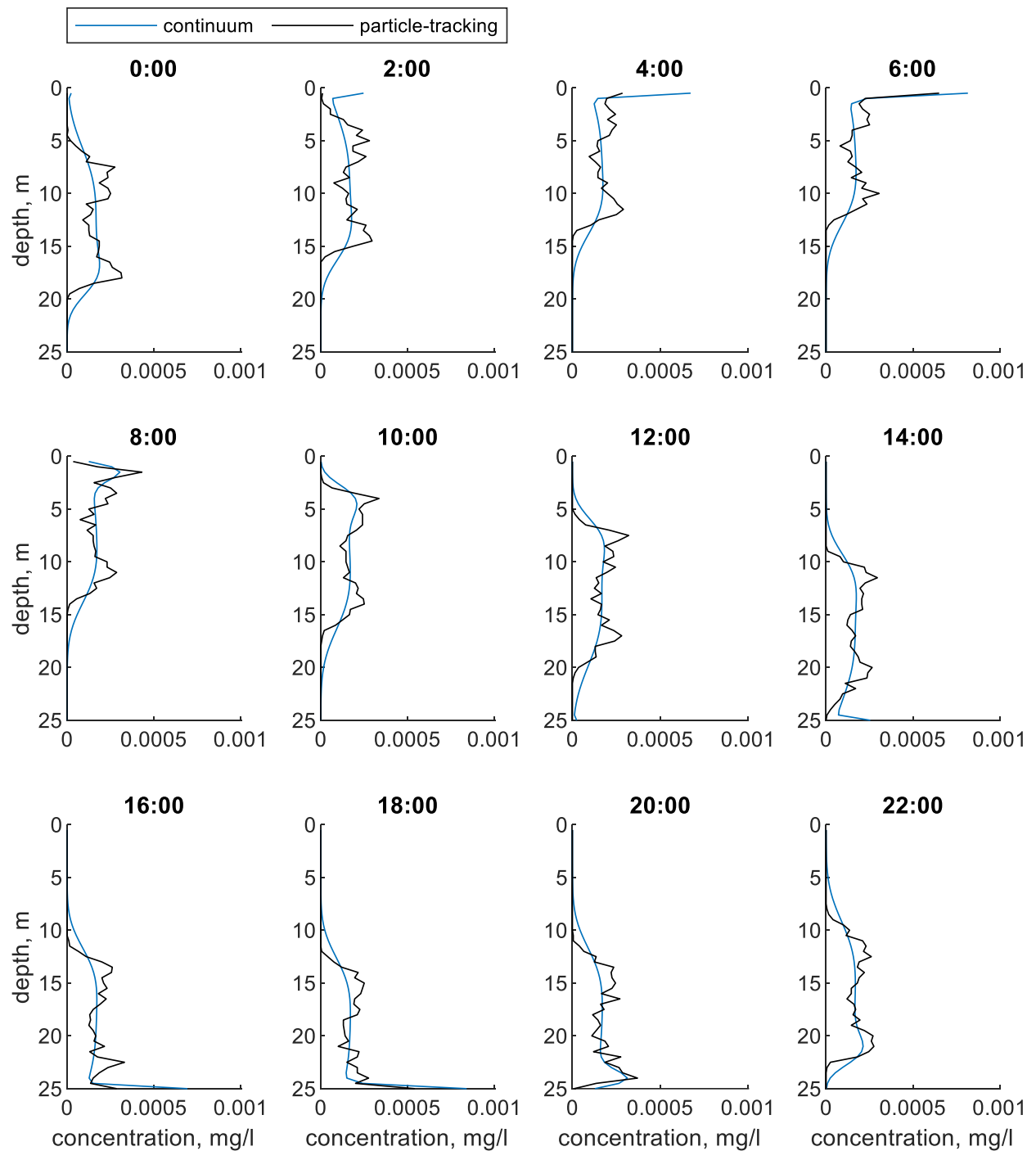

Figure 2-21 Concentration profiles at two-hour intervals predicted by preliminary Dodson (1990) models in continuum and particle-tracking frameworks

The model of Andersen and Nival (1991) predicted different behavior than the Dodson (1990) model. Instead of a gradual rise and fall throughout the day, zooplankton is predicted to stay at the bed throughout the photoperiod, rise to the surface at dusk, and remain there before descending at dawn (Figure 2-22 \& Figure 2-23). The same behavior is predicated by models in the continuum and particle- 
tracking frameworks. However, by the end of the fifth day in the simulation, concentrations are higher at the surface in the continuum framework and more diffused at mid-depth. This is due to the initial conditions of the model: concentration was initially uniformly distributed over the depth of the model grid, and the start time of the simulation corresponded to midnight. The modeled concentration moves down at dawn and forms a peak at the bed. A similar peak forms at the surface eventually, but this takes several days due to the distance that must be traveled from the bed to the surface and the migration speed. The same effect would be seen in the model using the particle-tracking framework, albeit after a longer time due to less numerical diffusion.

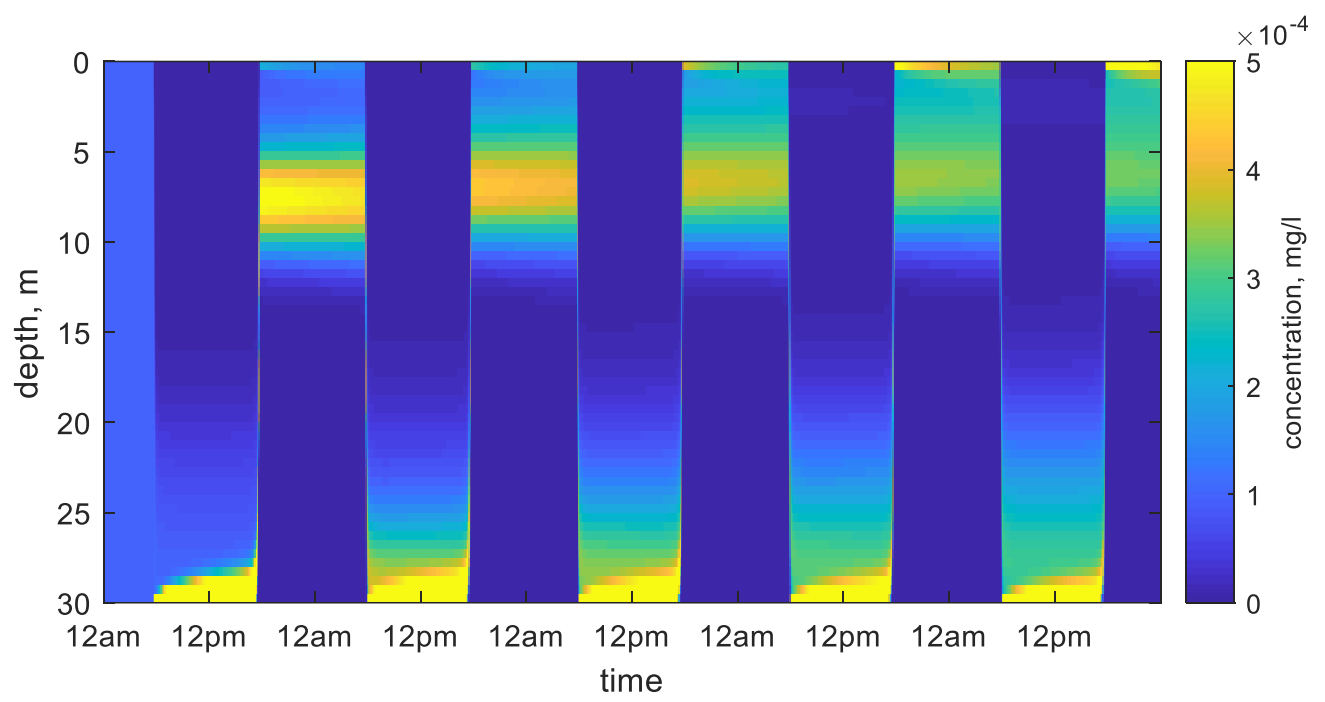

Figure 2-22 Five-day time series of concentration at depth predicted by preliminary Andersen \& Nival (1991) model (continuum) 


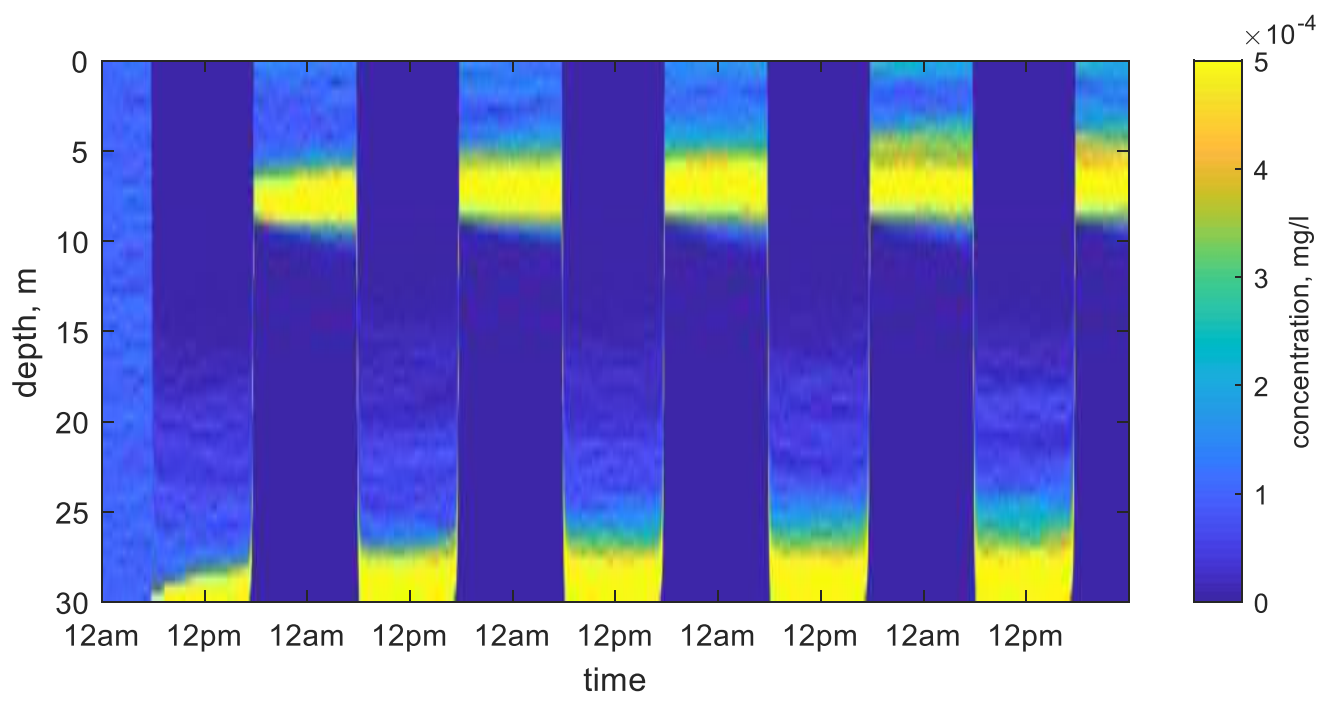

Figure 2-23 Five-day time series of concentration at depth predicted by preliminary Andersen \& Nival (1991) model (particle-tracking) 

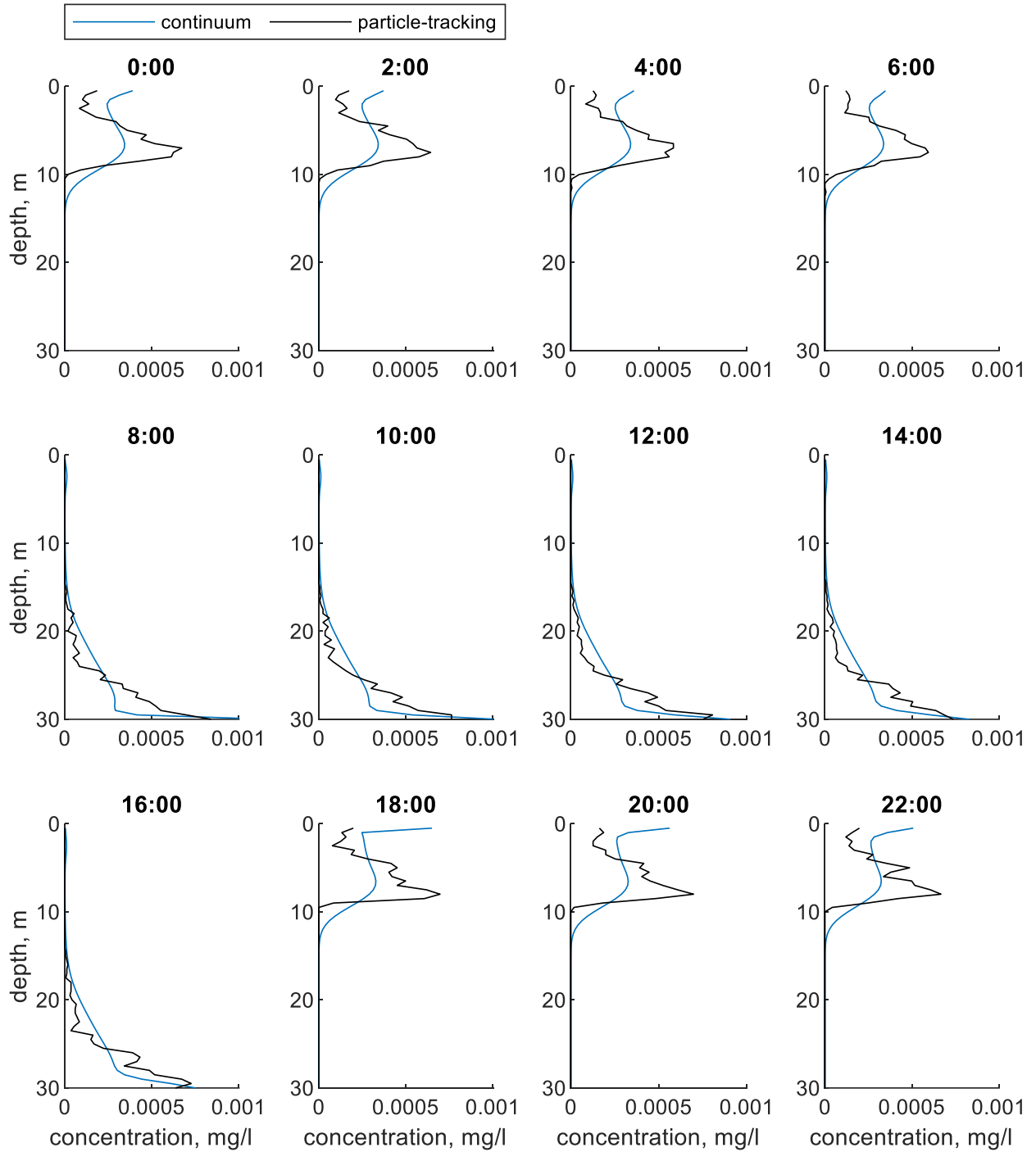

Figure 2-24 Concentration profiles at two-hour intervals predicted by preliminary Andersen \& Nival (1991) models in continuum and particle-tracking frameworks

The model using equations from Richards et al. (1996) predicts a much more homogenous distribution than the other two zooplankton models (Figure 2-25 \& Figure 2-26). Concentration reaches a minimum at the surface between dawn and dusk, when it is maximum at the bed. The opposite is true from dusk to dawn. This 
model predicts the same sudden movement at dawn and dusk as the model of Andersen and Nival (1991). Little variation can be seen in profile plots comparing the two frameworks outside of these times (Figure 2-27).

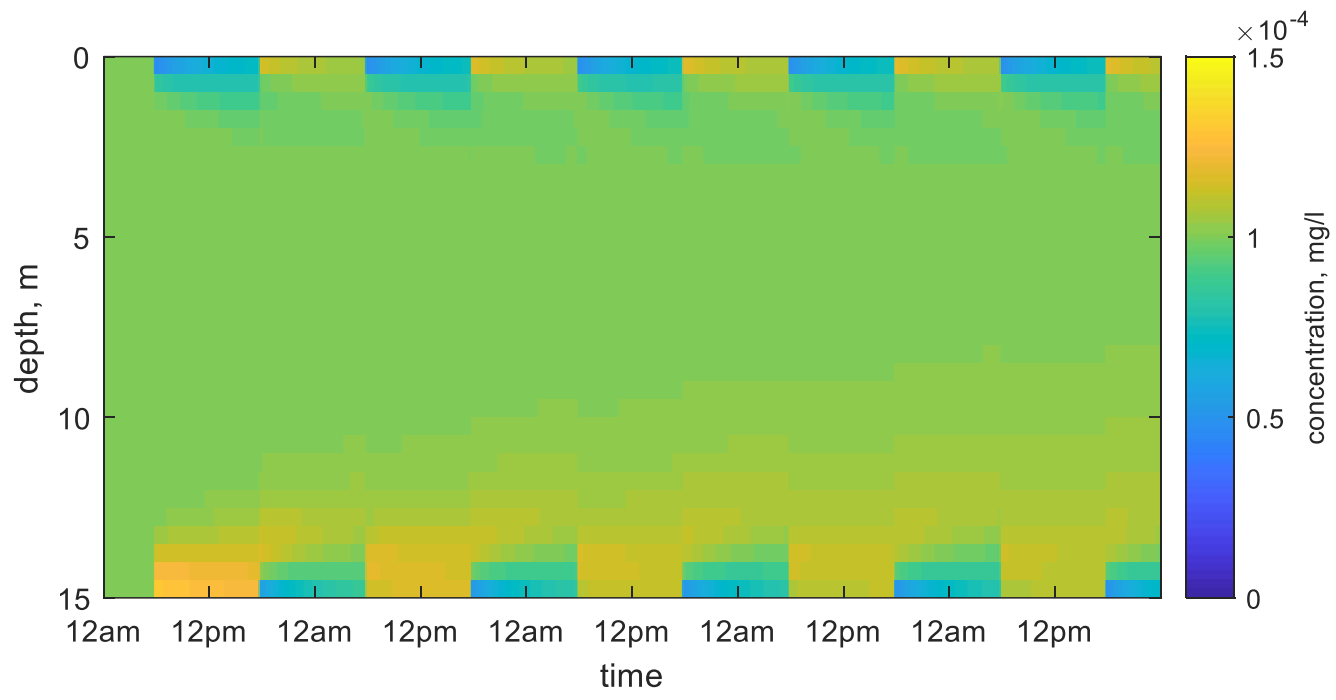

Figure 2-25 Five-day time series of concentration at depth predicted by preliminary Richards et al. (1996) model (continuum)

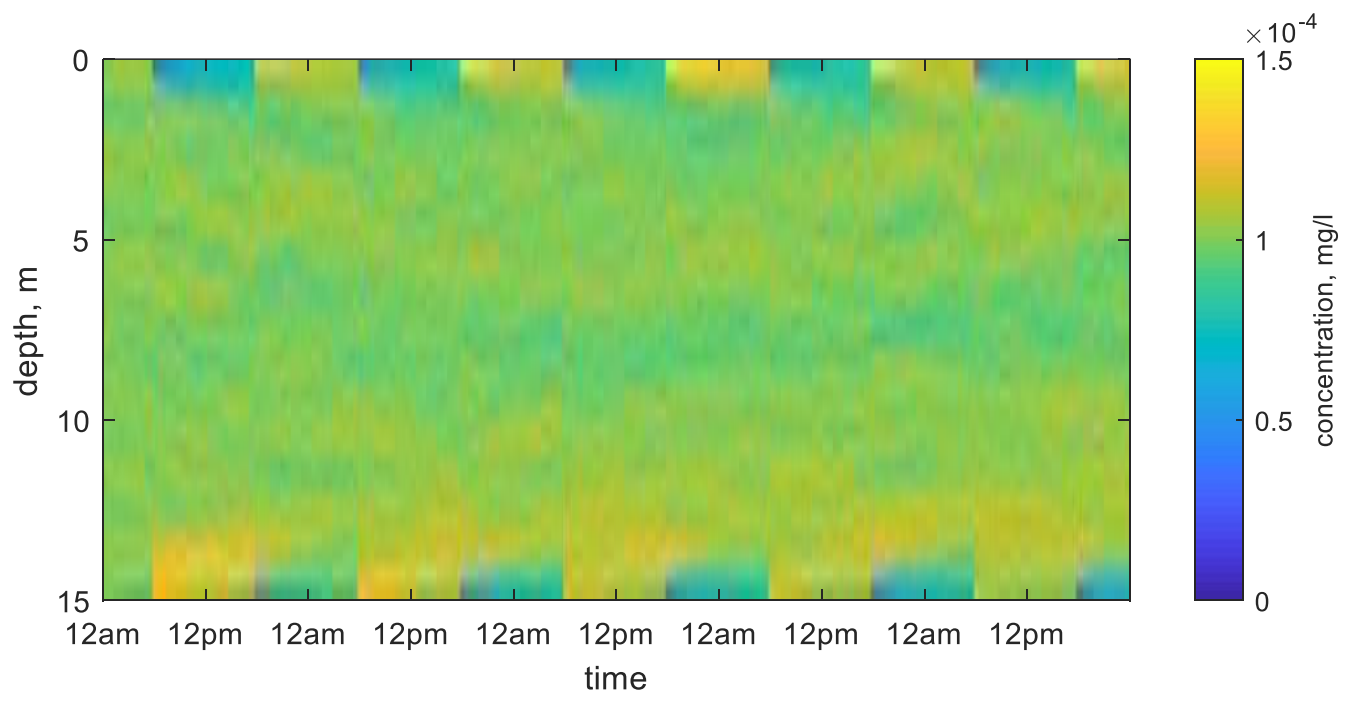

Figure 2-26 Five-day time series of concentration at depth predicted by preliminary Richards et al. (1996) model (particle-tracking) 

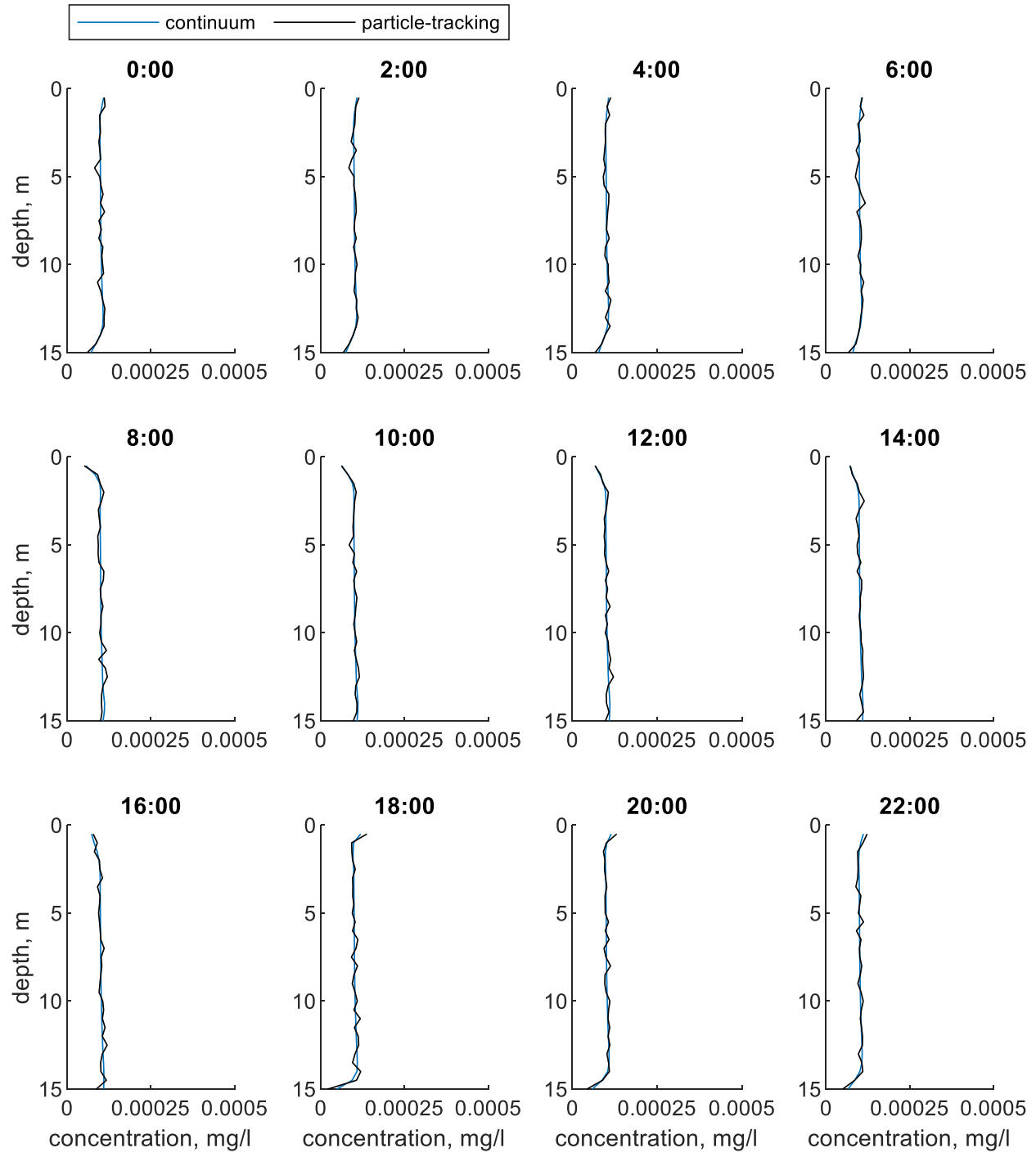

Figure 2-27 Concentration profiles at two-hour intervals predicted by preliminary Richards et al. (1996) models in continuum and particle-tracking frameworks

\subsection{Next Steps}

The preliminary models presented here show how each vertical migration model of cyanobacteria and zooplankton performs using generic input data in both continuum and particle-tracking frameworks. The cyanobacteria models using dynamic velocity equations predicted different concentration distributions over 
time between the two frameworks. Cyanobacteria models using predefined velocity equations and zooplankton models showed similar results between the two frameworks. All were able to show a daily vertical migration pattern characteristic of their respective organisms.

The next step in refining these models is testing them with real input data and measured concentration profiles. Cyanobacteria is the primary focus of this study because of its timely importance to water quality in lakes and reservoirs. Therefore, the following chapters focus on refining the cyanobacteria models and incorporating them into CE-QUAL-W2. However, zooplankton migration is an important aspect of water-quality modeling and the preliminary work done on zooplankton models here can still provide a basis for future model development. Further experimentation with these models could lead to adjusting the model parameters to better fit certain environmental settings or species. 


\section{Chapter 3: Comparison to Field Data}

\subsection{Selected Field Studies}

The models of cyanobacteria vertical migration described in Chapter 2 were applied to data from two published field studies. The first is a study by Cui et al. (2016). This study was done in Shennong Stream, a tributary of the Yangzhe River in the Three Gorges Reservoir complex, in China's Hubei Province. Water samples were taken at depth intervals of one meter every two hours on July 10-12, 2014 and analyzed for chlorophyll a concentration. Additional samples taken near the surface, at mid depth, and near the bed were analyzed for phytoplankton species composition, which indicated that almost $90 \%$ of the phytoplankton in the study areas belonged to the cyanobacteria genus Microcystis. This provided a basis for using chlorophyll a concentration to approximate Microcystis concentration. Two study sites were sampled: an 11-metter deep site in an enclosure protected from water currents and a 15-meter deep area in the open water. Solar irradiance above the water surface was measured every two hours, as was data used to calculate the light attenuation coefficient in each location. The published study includes solar measurements, calculated light attenuation coefficients, chlorophyll a concentration profiles, and calculated mean residence depth (MRD) of chlorophyll a concentration at the two-hour sampling intervals.

The other study used was performed by Wang et al. (2011) in another part of the Three Gorges Reservoir, Xiangxi Bay. In this study, hourly chlorophyll $a$ 
measurements were taken at depth intervals of 0.5 meters to a depth of nine meters on July 1, July $2-3$, and July 7,2008 . On July 3 , water samples were taken at six different depth intervals and analyzed for phytoplankton species composition. The results showed that $49.0 \%-83.2 \%$ of phytoplankton biomass (mg/l) and 83.7\%$94.8 \%$ of phytoplankton density ( $10^{7}$ cells/l) was due to Microcystis aeruginosa. Solar irradiance measured at the surface was also recorded every hour. Both these data are presented in the published study, as well as calculated MRD and depth of maximum chlorophyll a concentration.

\subsection{Methods}

The seven models using the continuum-framework and five models using the particle-tracking framework described in Chapter 2 were each applied to the two field study datasets. Chlorophyll a concentration was used as a proxy for Microcystis concentration. Parameter values were chosen from within reasonable ranges based on literature and adjusted to calibrate each model. The ranges of parameter values used for both field data applications are given in Table 3-1. Initial colony density values were assumed to vary exponentially from the surface to the bed (Equation 61), following a similar pattern to light decay with depth.

$$
\rho_{0_{i}}=\rho_{0_{S}}+\left(\rho_{0_{B}}-\rho_{0_{S}}\right)\left(1-e^{-z_{i}}\right)
$$

Here, $\rho_{0_{S}}$ is the initial colony density at the surface and $\rho_{0_{B}}$ is the initial colony density at the bed. 
Table 3-1 Ranges of values used in model applications to field study data

\begin{tabular}{|c|c|c|}
\hline Variable & Description & Value Range \\
\hline$A, \mathrm{~m}$ & Migration amplitude & $0.2-1.23$ \\
\hline$\phi, \mathrm{rad}$ & Phase offset & $\pi$ \\
\hline$C$ & Light attenuation calibration coefficient & $0.05-0.13$ \\
\hline$\mu_{g, m a x}, \mathrm{day}^{-1}$ & Maximum growth rate & $0.7-1.0$ \\
\hline$\mu_{m}, \mathrm{day}^{-1}$ & Mortality rate & $0.06-0.25$ \\
\hline$\mu_{e}, \mathrm{day}^{-1}$ & Excretion rate & 0.04 \\
\hline$\mu_{r}, \mathrm{day}^{-1}$ & Respiration rate & 0.04 \\
\hline$I_{s}, \mathrm{~W} \mathrm{~m}^{-2}$ & Saturating light intensity & $100-150$ \\
\hline$c_{1}, \mathrm{day}^{-1}$ & Coefficient of density increase for light function model & $0.00545-0.02$ \\
\hline$c_{2}, \mathrm{day}^{-1}$ & Coefficient of density decrease for light function model & $0.00145-0.00518$ \\
\hline$r_{c}, \mathrm{~mm}^{-3}$ & Colony radius & $15-64$ \\
\hline$\rho_{m i n}, \mathrm{~kg} \mathrm{~m}^{-3}$ & Minimum colony density & $920-980$ \\
\hline$\rho_{m a x}, \mathrm{~kg} \mathrm{~m}^{-3}$ & Maximum colony density & $140-185$ \\
\hline$\rho_{0, S}, \mathrm{~kg} \mathrm{~m}^{-3}$ & Initial colony density at surface & $930-1080$ (continuum) \\
\hline$\rho_{0, B}, \mathrm{~kg} \mathrm{~m}^{-3}$ & Initial colony density at bed & $920-980$ (particles) \\
\hline$\rho_{i, S}, \mathrm{~kg} \mathrm{~m}^{-3}$ & Minimum initial density at surface for Visser et al. & $995-1010$ (particles) \\
\hline$\rho_{i, B}, \mathrm{~kg} \mathrm{~m}^{-3}$ & Minimum initial density at bed for Visser et al. (1997) & $980-1080$ \\
\hline$\rho_{*}, \mathrm{~kg} \mathrm{~m}^{-3}$ & Correction for density decrease equation & $975-980$ \\
\hline$k, \mathrm{day}^{-1}$ & Time decay constant for averaging past densities & 67 \\
\hline & & 5 \\
\hline
\end{tabular}

An adjustment was made to the Visser et al. (1997) model to account for

adaptations from the particle-tracking to the continuum framework. If the

calculated value of $\rho_{i}$, the density used to calculate density decrease in the dark, is

below a minimum value, it is increased to that minimum value. This varies with

depth by Equation 62,

$$
\rho_{i_{i}}=\rho_{i_{S}}+\left(\rho_{i_{B}}-\rho_{i_{S}}\right)\left(\frac{z_{i}}{H}\right)
$$


where $\rho_{i_{S}}$ is the minimum allowable value of $\rho_{i}$ at the surface and $\rho_{i_{B}}$ is the minimum allowable value at the bed.

Because this was an application to a real system, growth kinetics were included in all models, as opposed to being ignored like they were in preliminary models. For the continuum framework, this was simply including the source-sink term from Equation 32 and applying it to each grid cell. For the particle-tracking framework, the growth equations were applied to the mass of a particle. The initial mass is determined by the initial colony density and radius. The total number of particles in each grid cell is determined using Equation 58, taking into account variations in initial colony density.

Input data for the Shennong Stream models included solar irradiance at the water surface and light attenuation coefficients calculated in the study. Initial conditions were set to the first recorded field measurement at each depth, taken at 8:00am on the first day of the study. This also determined the simulation start time. In all models of Shennong Stream, a grid cell height $(\Delta z)$ of 0.2 meters was used and the model timestep $(\Delta t)$ was 60 seconds. Water current was not modeled, and water was assumed to be quiescent. For the enclosure site, the vertical diffusion coefficient $\left(D_{z}\right)$ was set to a constant $10^{-5} \mathrm{~m}^{2} \mathrm{~s}^{-1}$ and in the open water site it was set to $10^{-4} \mathrm{~m}^{2} \mathrm{~s}^{-1}$. This was meant to represent the differences in turbulent mixing between the two sites due to assumed differences in water currents. 
Chlorophyll a measurements at depth intervals of one meter and time intervals of two hours were obtained from the study (Figure 3-1), as were calculated MRDs at the same time intervals (Figure 3-2). These data were used to test and calibrate the models of cyanobacteria vertical migration.
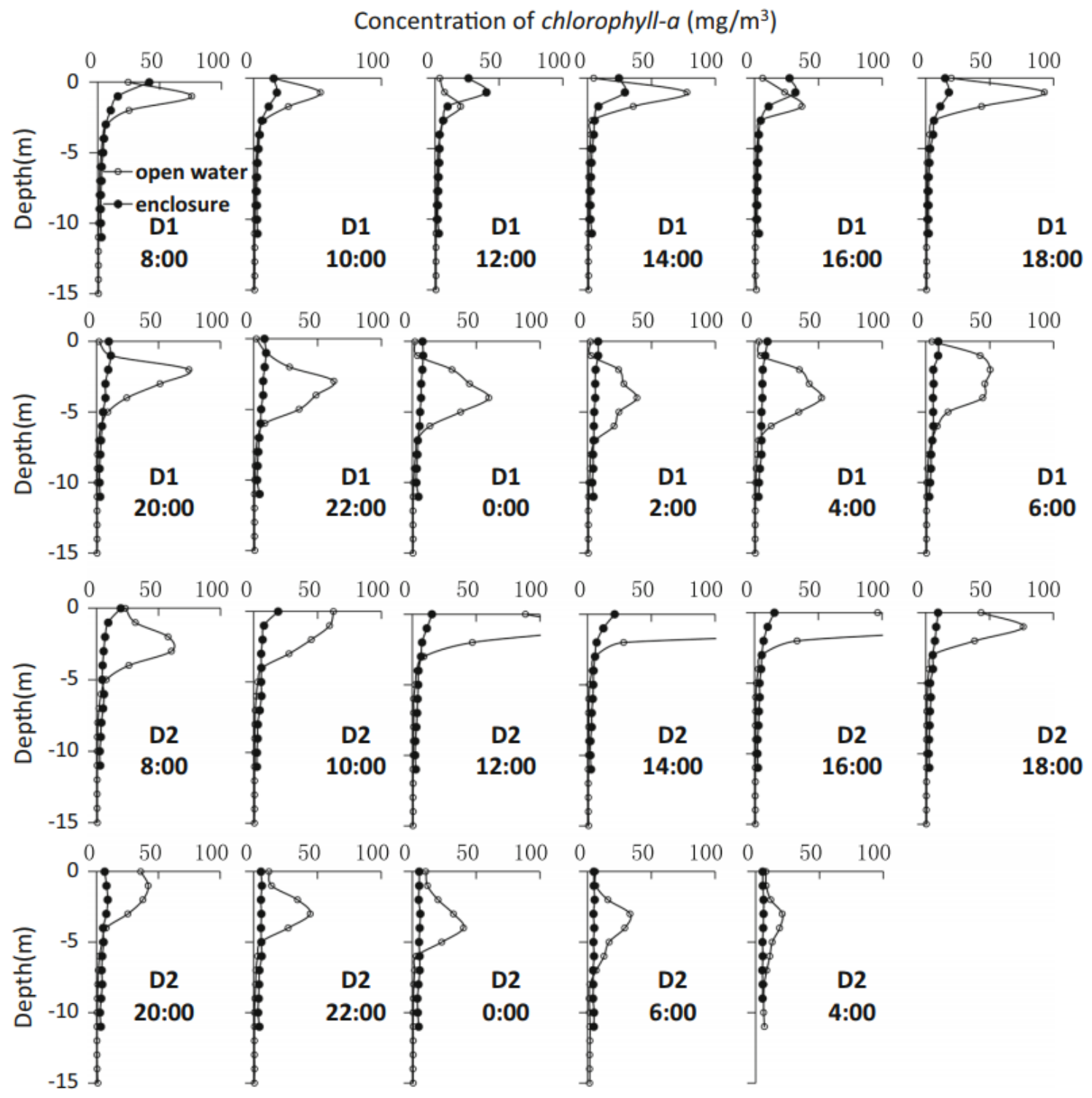

Figure 3-1 Chlorophyll a concentration profiles measured in Shennong stream enclosure (dark dots) and open water (light dots) sites, extracted from Cui et al. (2016) 


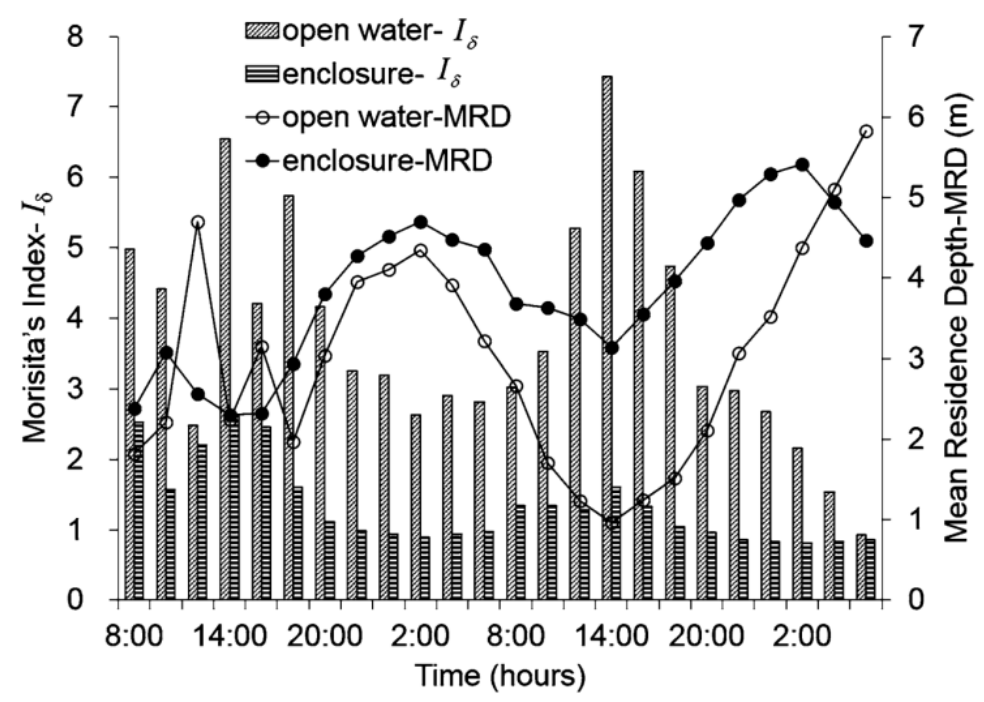

Figure 3-2 Morisita's Index (I, bars) and mean residence depth (MRD, dots) calculated from chlorophyll a profiles measured in Shennong Stream open water and enclosure sites, extracted from Cui et al. (2016)

Absolute mean errors (AMEs) of all models compared to field data was calculated for each of the metrics and are presented here (Table 3-2, Table 3-3, Table 3-4, \& Table 3-5). Predictions of chlorophyll a concentration were prioritized over predictions of MRD. The amount of cyanobacteria (represented by chlorophyll a) at each depth in the water column is of interest here because it is a primary measurement of cyanobacteria distribution, while MRD is a derived metric. Chlorophyll a concentration errors are averages of AMEs from chlorophyll $a$ concentration profiles. Additional model-data comparisons of chlorophyll a profiles for all models can be found in Appendix A.

Inputs for the Xiangxi Bay field study application were solar irradiance measured at the water surface and initial chlorophyll a concentrations at $0.5 \mathrm{~m}$ depth intervals (Figure 3-3), both reported in the study published by Wang et al. 
(2011). Quantitative model-data comparisons were made with hourly MRD (Figure 3-4) and depth of maximum chlorophyll a concentration (Figure 3-3) reported in the study. Similar to the Shennong Stream study, predictions of depth of maximum chlorophyll a concentration were prioritized over predictions of MRD. Qualitative comparisons were made with contour plots similar to Figure 3-3 (Appendix B).
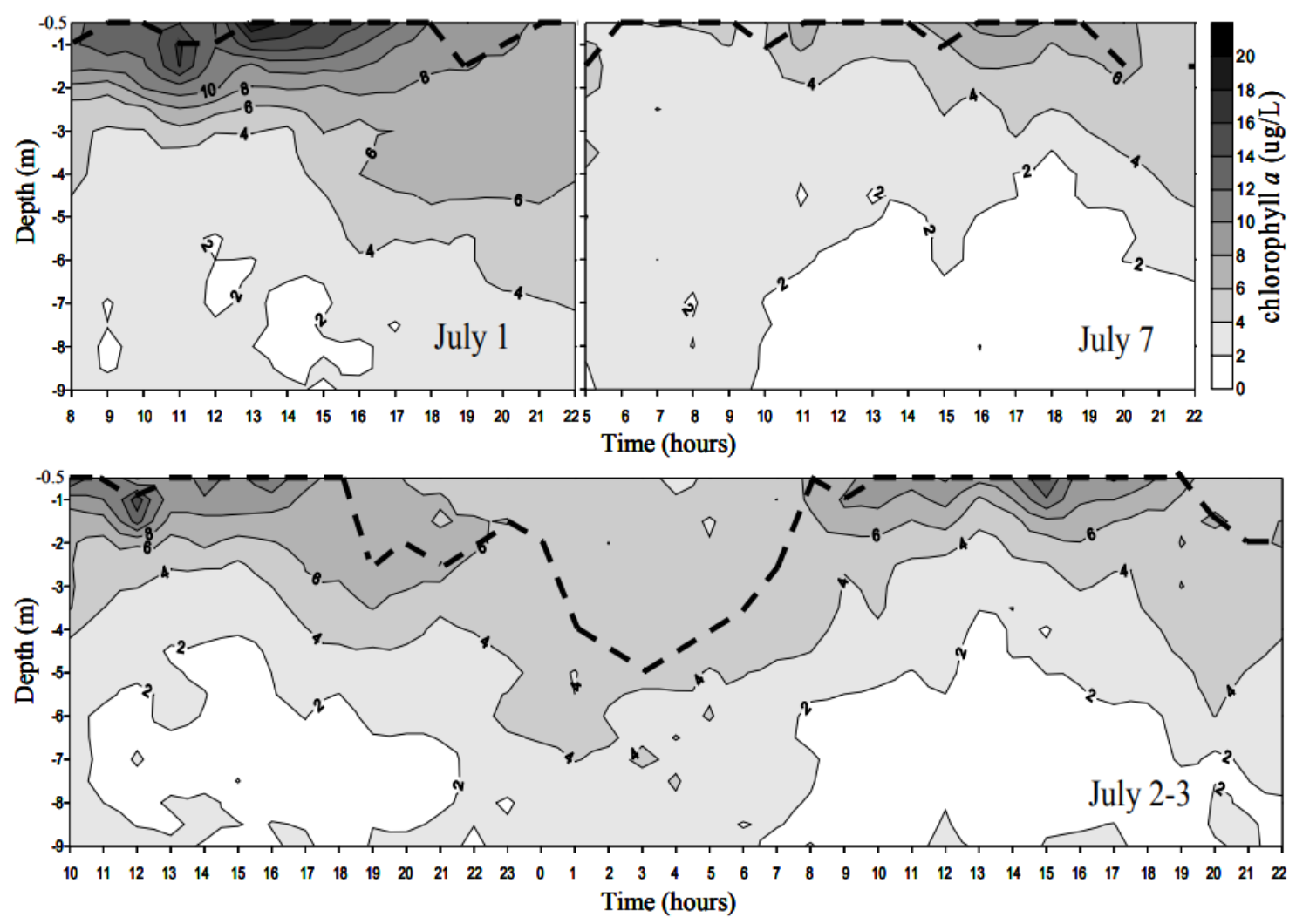

FIGURE 4 - Depth-time isopleths of chlorophyll $a$ and the depth of maximal chlorophyll $a$ in the water column (dashed lines)

Figure 3-3 Time series of chlorophyll a contours measured at Xiangxi Bay with depth of maximum chlorophyll a concentration shown by dashed line, extracted from Wang et al. (2011) 


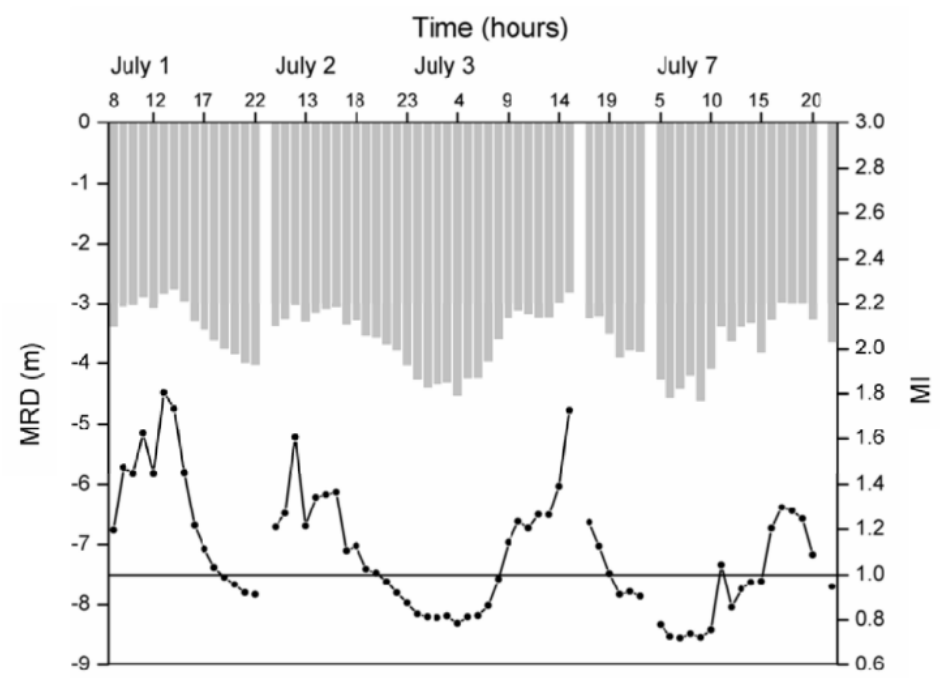

Figure 3-4 Mean residence depth (MRD, bars) and Morisita's Index (MI, dotted line) calculated from chlorophyll a data from Xiangxi Bay, extracted from Wang et al. (2011)

The light attenuation coefficient was set to $0.5 \mathrm{~m}^{-1}$ for all models. This was done in part to test the models with a lower light attenuation coefficient value, as the values in the Shennong Stream study were relatively high. Each model was run with two different values of $D_{z}, 10^{-5} \mathrm{~m}^{2} \mathrm{~s}^{-1}$ and $10^{-4} \mathrm{~m}^{2} \mathrm{~s}^{-1}$. This allowed for comparisons between the Shennong Stream application and the Xiangxi Bay application in terms of vertical diffusion.

\subsection{Results}

\subsubsection{Shennong Stream}

\section{Enclosure}

The chlorophyll a profiles taken in the enclosure site in Shennong stream show subtle changes in shape throughout the study period (Figure 3-1). A subsurface peak can be seen on the morning of the first day; after that the profile becomes more diffuse and then develops a surface maxima on the morning of the 
second day. The MRD shows a distinct diurnal sinusoid pattern, with an amplitude of approximately 1.5 meters (Figure 3-2).

In the continuum modeling framework, the two predefined velocity models resulted in the lowest AME values for both MRD and chlorophyll a concentration (Table 3-2). These models were able to represent the sinusoidal pattern of the MRD seen in the field data (Figure 3-5). The dynamic velocity models did not predict MRD as well as the predefined velocity models. In most of the dynamic velocity models, the MRD did not show the distinct sinusoidal pattern seen in the data and instead showed little variation over time (Figure 3-6). The predefined velocity models also captured chlorophyll a concentration profiles better than the dynamic velocity models at this site (Figure 3-7 \& Figure 3-8). Most of the dynamic velocity models failed to predict concentration further down in the water column the second day, with the exception of the light function model with time decay (Figure 3-8).

Table 3-2 Error statistics from models of Shennong Stream enclosure site (continuum)

\begin{tabular}{|l|r|l|r|}
\hline \multicolumn{2}{|c|}{ Mean Residence Depth } & \multicolumn{2}{c|}{$\begin{array}{c}\text { Chlorophyll a concentration } \\
\text { (profile average) }\end{array}$} \\
\hline \multicolumn{1}{|c|}{ Model } & AME, $\mathrm{m}$ & \multicolumn{1}{c|}{ Model } & AME, mg m ${ }^{3}$ \\
\hline Belov \& Giles (1997) & 0.799 & Belov \& Giles (1997) & 2.549 \\
\hline Time-varying velocity & 1.074 & Time-varying velocity & 2.871 \\
\hline Light function with time decay & 1.252 & Light function with time decay & 3.201 \\
\hline Growth kinetics with time decay & 1.318 & Visser et al. (1997) & 3.271 \\
\hline Visser et al. (1997) & 1.338 & Light function & 3.378 \\
\hline Growth kinetics & 1.348 & Growth kinetics & 3.446 \\
\hline Light function & 1.359 & Growth kinetics with time decay & 3.522 \\
\hline
\end{tabular}



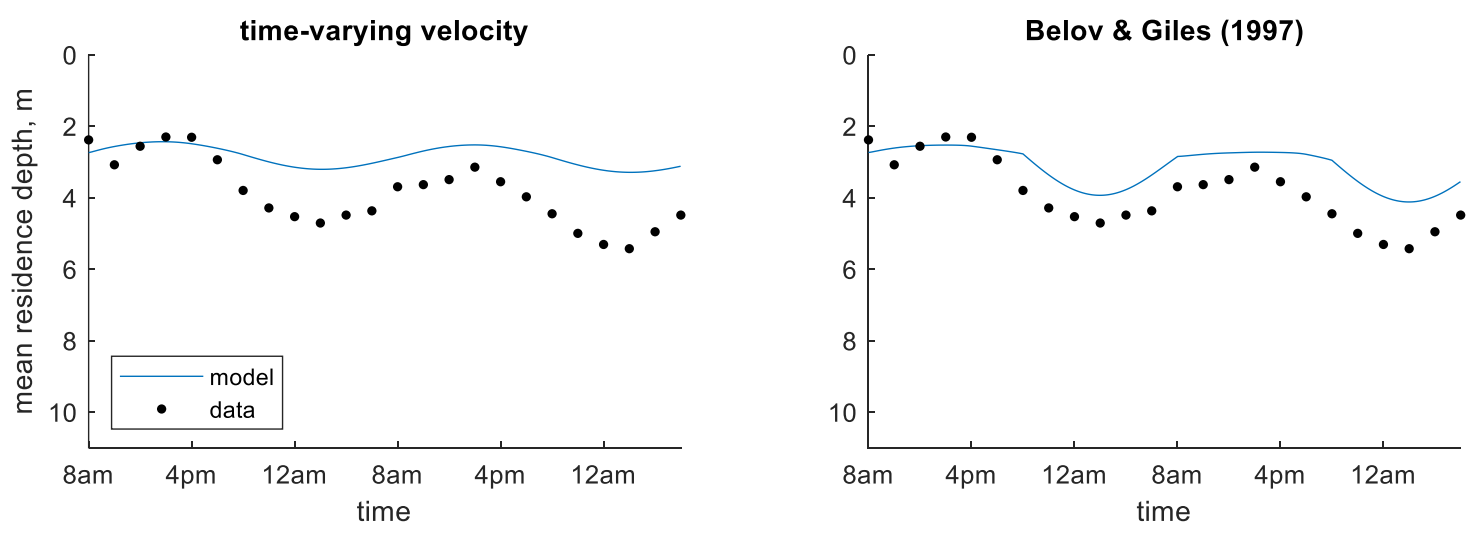

Figure 3-5 Time series of observed and predicted mean residence depth of chlorophyll a concentration in Shennong Stream enclosure site using predefined velocity models (continuum) 

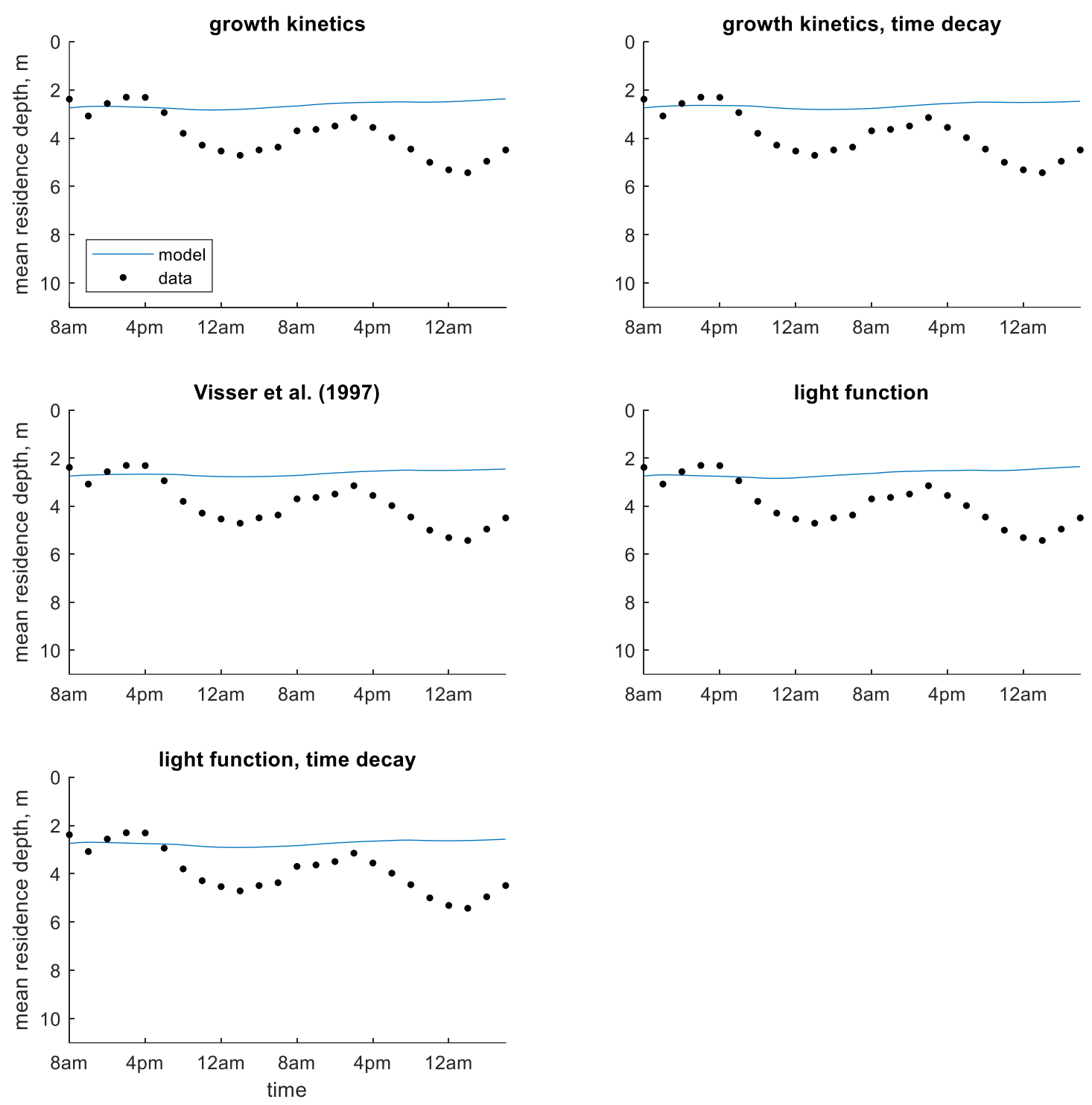

Figure 3-6 Time series of observed and predicted mean residence depth of chlorophyll a concentration in Shennong Stream enclosure site using dynamic velocity models (continuum) 

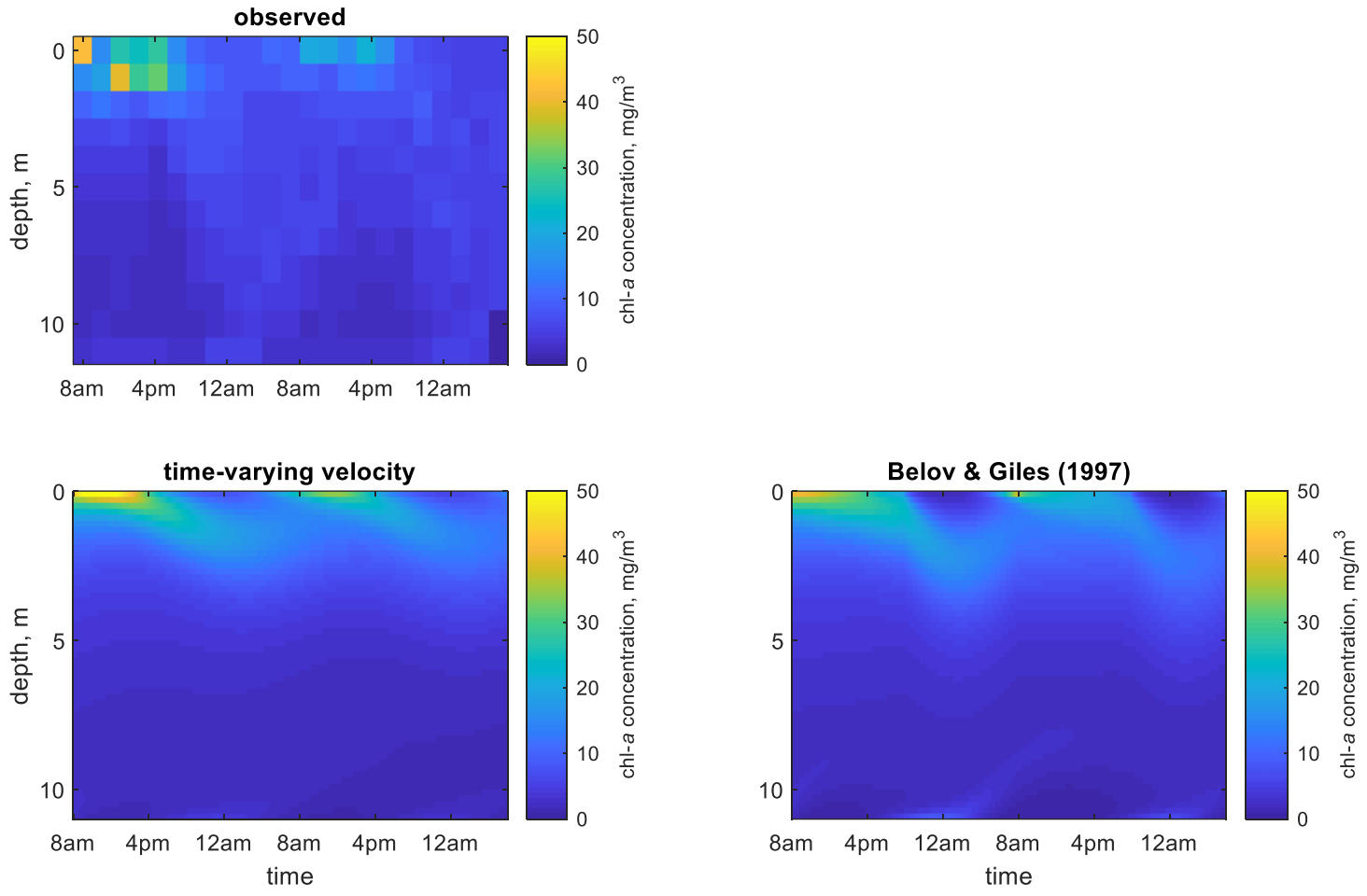

Figure 3-7 Time series of observed and predicted chlorophyll a concentration in Shennong Stream enclosure site using predefined velocity models (continuum) 

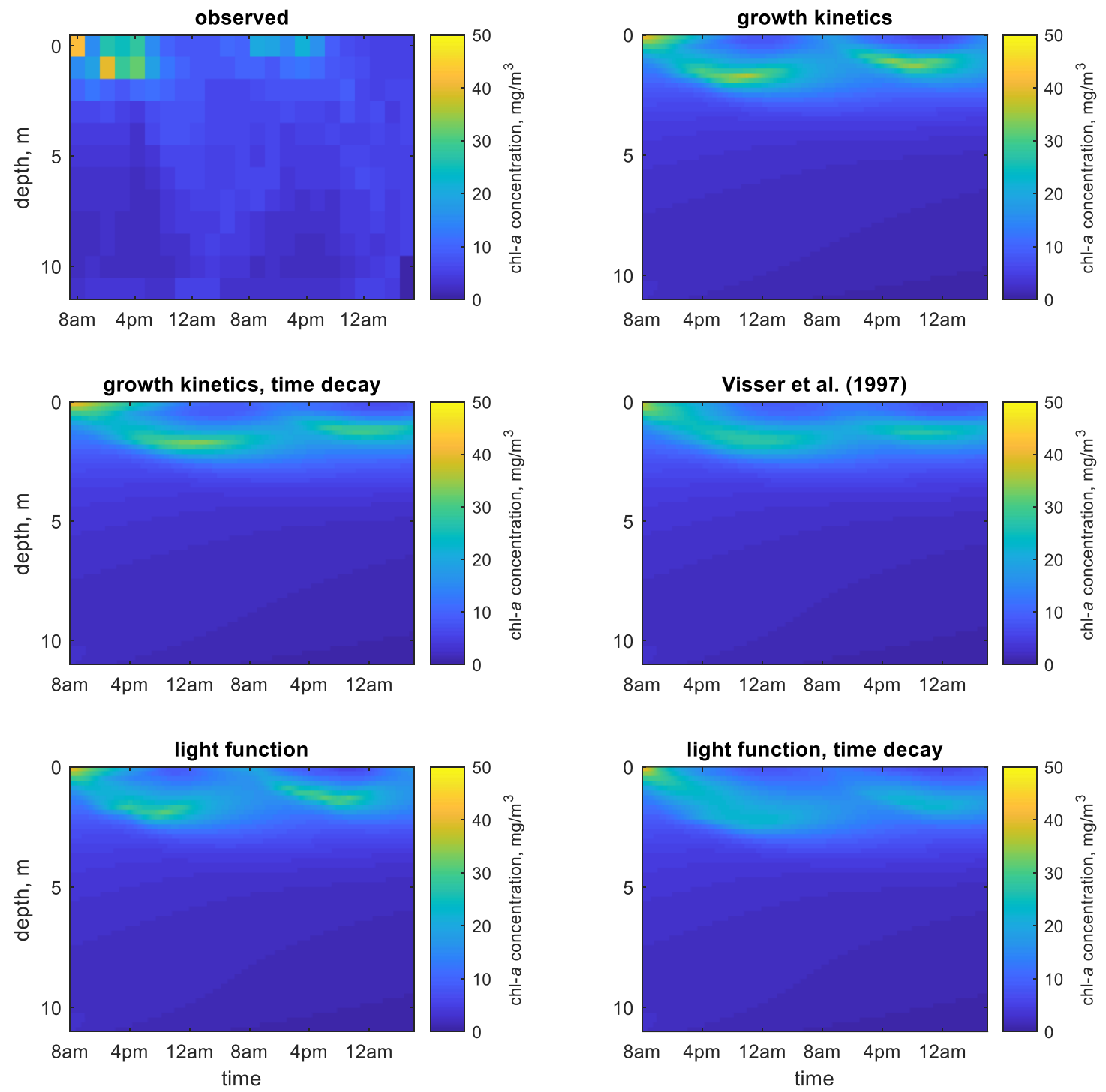

Figure 3-8 Time series of observed and predicted chlorophyll a concentration in Shennong Stream enclosure site using dynamic velocity models (continuum)

Somewhat different results were obtained from models applied to the Shennong Stream enclosure site using the particle-tracking framework. Among these models, MRD was best predicted by the dynamic velocity models, especially the light function and growth kinetics models (Table 3-3). However, the predefined velocity models still follow the correct shape of the MRD over the entire study 
period (Figure 3-9), while the dynamic velocity models tend to under-predict the MRD during the final several hours (Figure 3-10). As with models in the continuum framework, chlorophyll a was best predicted by the Belov and Giles (1997) model. In the vertical concentration time-series plots, the predefined velocity models show more diffusion than the dynamic velocity models (Figure 3-11 \& Figure 3-12).

The most notable difference between the continuum and particle-tracking frameworks for this study can be seen in the dynamic velocity models. These models resulted in AMEs of 1.25-1.36 m for MRD in the continuum framework (Table 3-2) and $0.60-0.77 \mathrm{~m}$ in the particle-tracking framework (Table 3-3). Little change was seen in chlorophyll a concentration predictions by dynamic velocity models or MRD predictions by predefined velocity models. However, AMEs for chlorophyll $a$ concentration predictions by predefined velocity models increased from 2.55 and $2.87 \mathrm{mg} / \mathrm{m}^{3}$ in the continuum framework to 2.80 and $3.18 \mathrm{mg} / \mathrm{m}^{3}$ in the particletracking framework.

Table 3-3 Error statistics from models of Shennong Stream enclosure site (particle-tracking)

\begin{tabular}{|l|r|l|r|}
\hline \multicolumn{2}{|c|}{ Mean Residence Depth } & \multicolumn{2}{c|}{$\begin{array}{c}\text { Chlorophyll a concentration } \\
\text { (profile average) }\end{array}$} \\
\hline \multicolumn{1}{|c|}{ Model } & AME, $\mathrm{m}$ & \multicolumn{1}{c|}{ Model } & AME, $\mathbf{~ m g ~ m}^{3}$ \\
\hline Light function & 0.599 & Belov \& Giles (1997) & 2.796 \\
\hline Growth kinetics & 0.613 & Time-varying velocity & 3.176 \\
\hline Visser et al. (1997) & 0.772 & Visser et al. (1997) & 3.205 \\
\hline Belov \& Giles (1997) & 0.795 & Light function & 3.273 \\
\hline Time-varying velocity & 1.127 & Growth kinetics & 3.925 \\
\hline
\end{tabular}



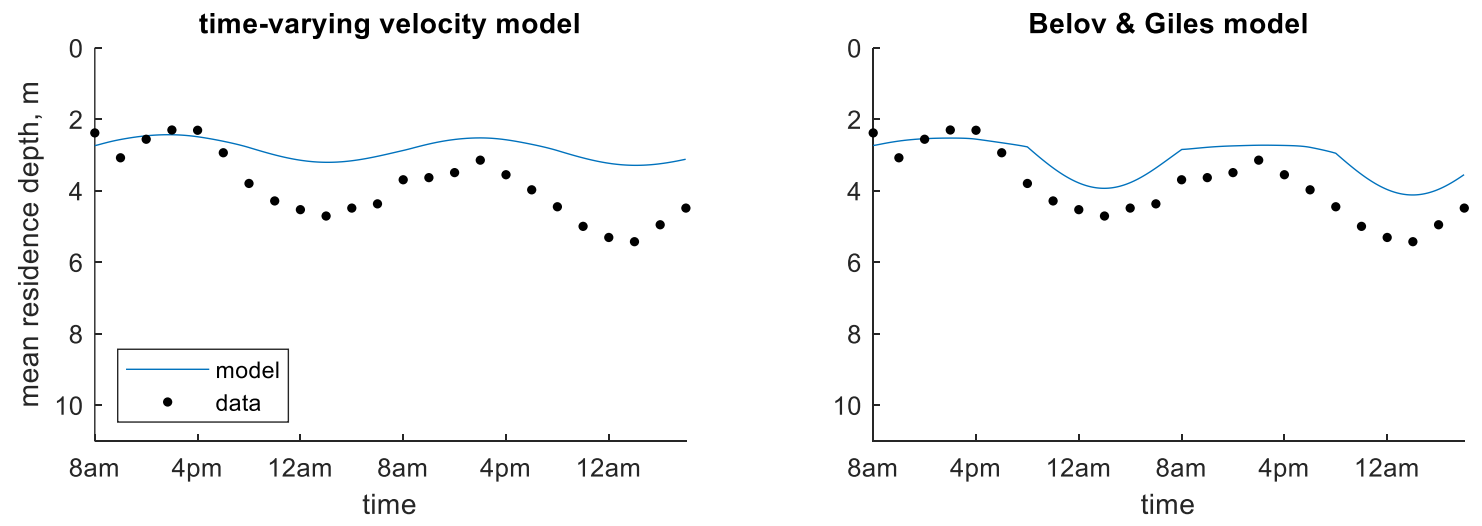

Figure 3-9 Time series of observed and predicted mean residence depth of chlorophyll a concentration in Shennong Stream enclosure site using predefined velocity models (particletracking)
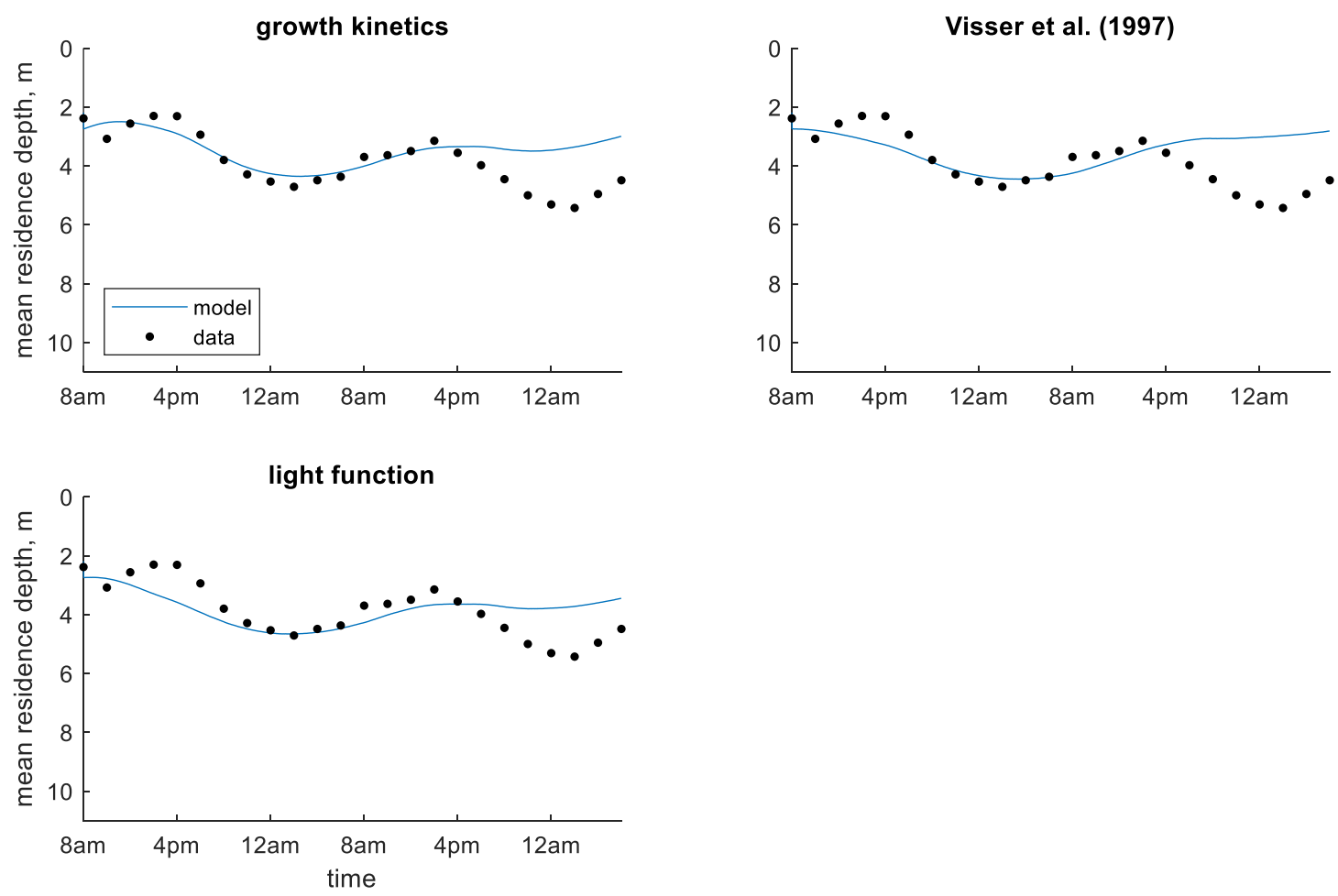

Figure 3-10 Time series of observed and predicted mean residence depth of chlorophyll a concentration in Shennong Stream enclosure site using dynamic velocity models (particle-tracking) 

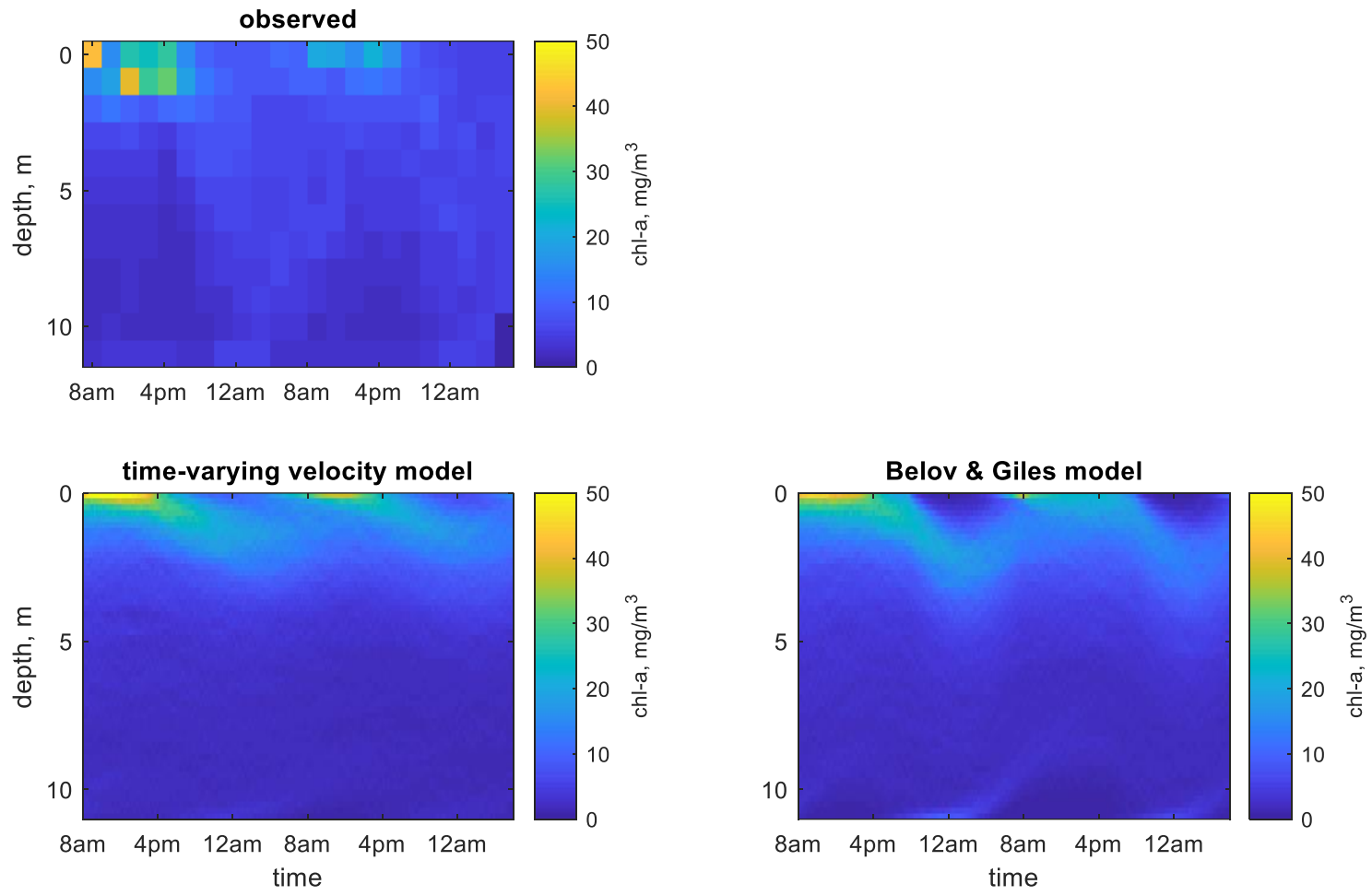

Figure 3-11 Time series of observed and predicted chlorophyll a concentration in Shennong Stream enclosure site using predefined velocity models (particle-tracking) 

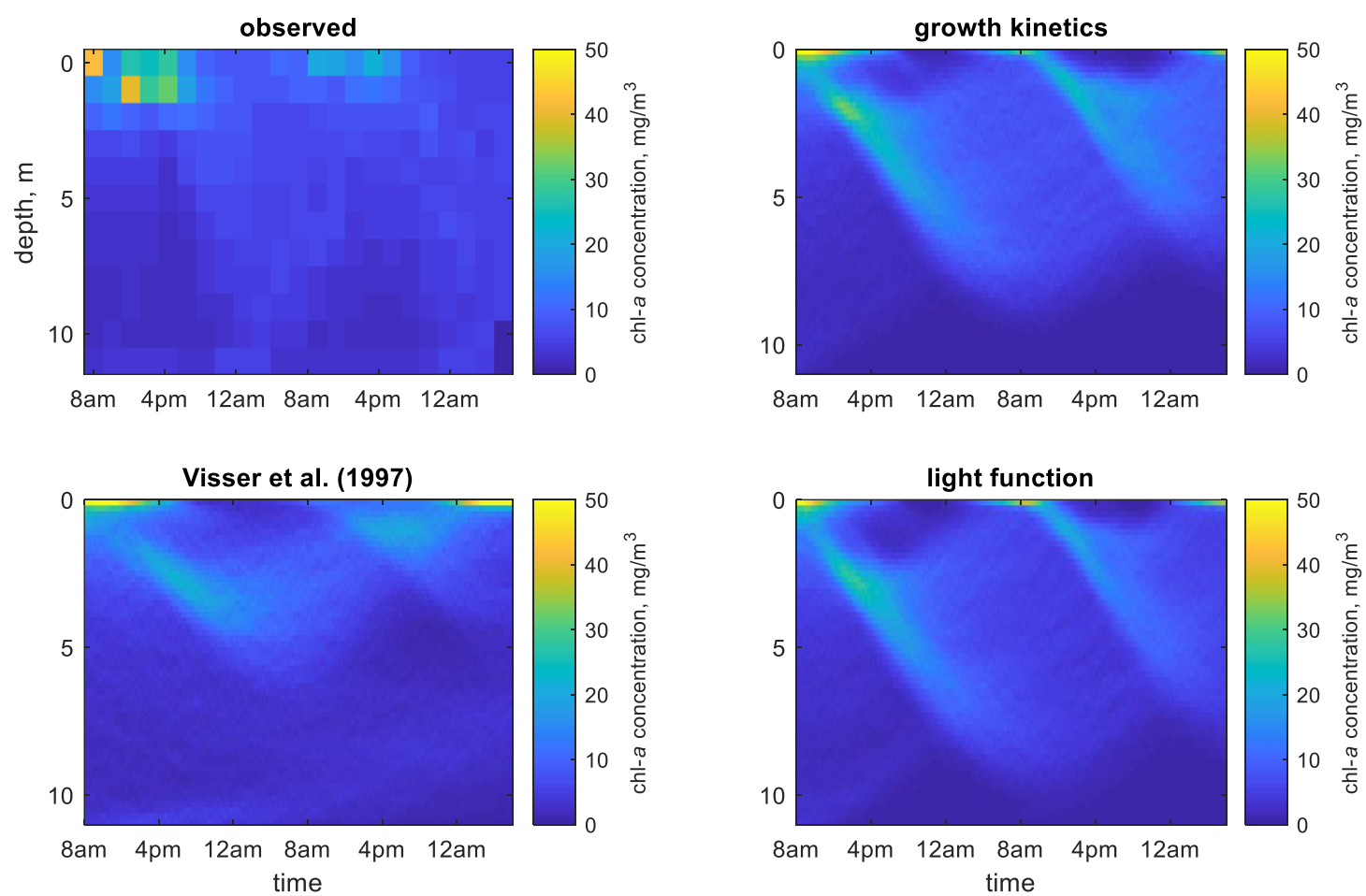

Figure 3-12 Time series of observed and predicted chlorophyll a concentration in Shennong Stream enclosure site using dynamic velocity models (particle-tracking)

\section{Open Water}

In the open water site in Shennong stream, concentration profile plots showed a more distinct shape, alternating between a subsurface peak and a surface maxima (Figure 3-1). As in the enclosure site, MRD generally followed a sinusoidal pattern. However, in the open water MRD moved closer to the surface on the second morning and continued to move downward at the end of the study period when the MRD in the enclosure had begun moving upward (Figure 3-2).

Of the models in the continuum framework, the dynamic velocity models predicted both MRD and chlorophyll a profiles better than the predefined velocity models, though only slightly (Table 3-4). However, a visual inspection of the MRD 
predictions show that the dynamic velocity models predict an almost constant MRD, while the predefined velocity models do better at capturing the shape of the MRD (Figure 3-13 \& Figure 3-14). The concentration time-series plots of chlorophyll $a$ show that concentration predictions are less diffuse than in the enclosure site, and the predefined velocity models predict that chlorophyll $a$ is more diffuse than the field data (Figure 3-15). The dynamic velocity models do not show as much of a change in diffusion after adjusting the vertical diffusion coefficient for the open water site, resulting in more accurate profile predictions (Figure 3-16).

Table 3-4 Error statistics from models of Shennong Stream open water site (continuum)

\begin{tabular}{|l|r|l|r|}
\hline \multicolumn{2}{|c|}{ Mean Residence Depth } & \multicolumn{2}{c|}{$\begin{array}{c}\text { Chlorophyll a concentration } \\
\text { (profile average) }\end{array}$} \\
\hline \multicolumn{1}{|c|}{ Model } & AME, $\mathrm{m}$ & \multicolumn{1}{c|}{ Model } & AME, mg m ${ }^{3}$ \\
\hline Growth kinetics with time decay & 1.064 & Growth kinetics with time decay & 7.193 \\
\hline Light function with time decay & 1.087 & Light function with time decay & 7.253 \\
\hline Light function & 1.087 & Visser et al. (1997) & 7.425 \\
\hline Visser et al. (1997) & 1.093 & Light function & 7.589 \\
\hline Growth kinetics & 1.096 & Growth kinetics & 7.735 \\
\hline Belov \& Giles (1997) & 1.113 & Time-varying velocity & 8.589 \\
\hline Time-varying velocity & 1.129 & Belov \& Giles (1997) & 8.645 \\
\hline
\end{tabular}
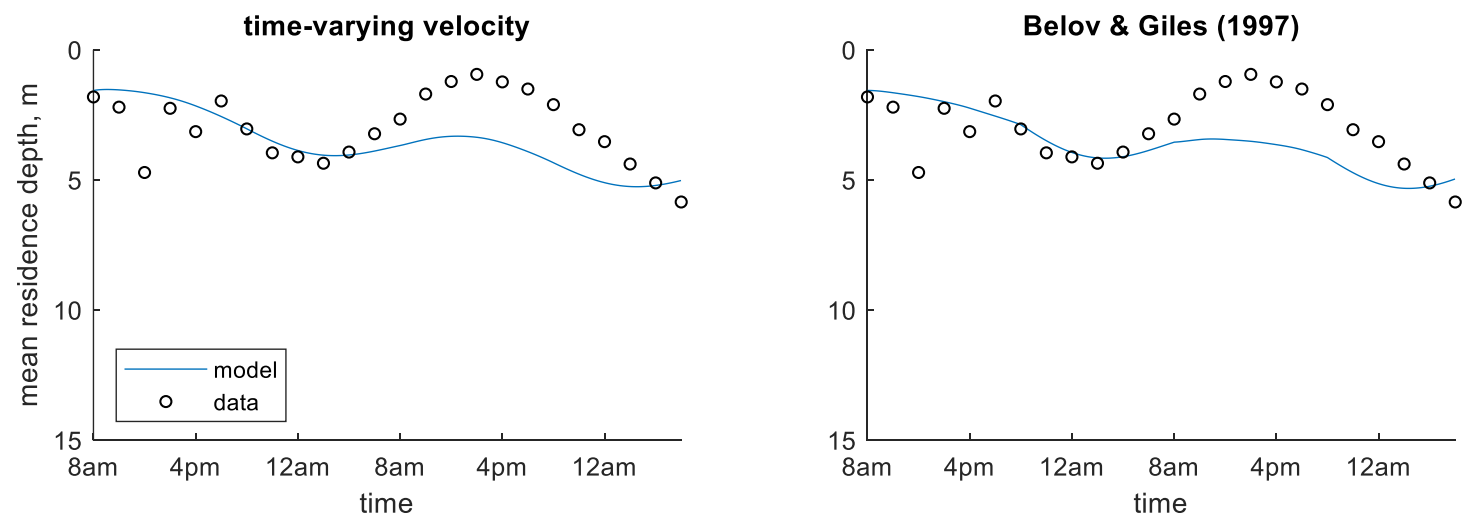

Figure 3-13 Time series of observed and predicted mean residence depth of chlorophyll a concentration in Shennong Stream open water site using predefined velocity models (continuum) 

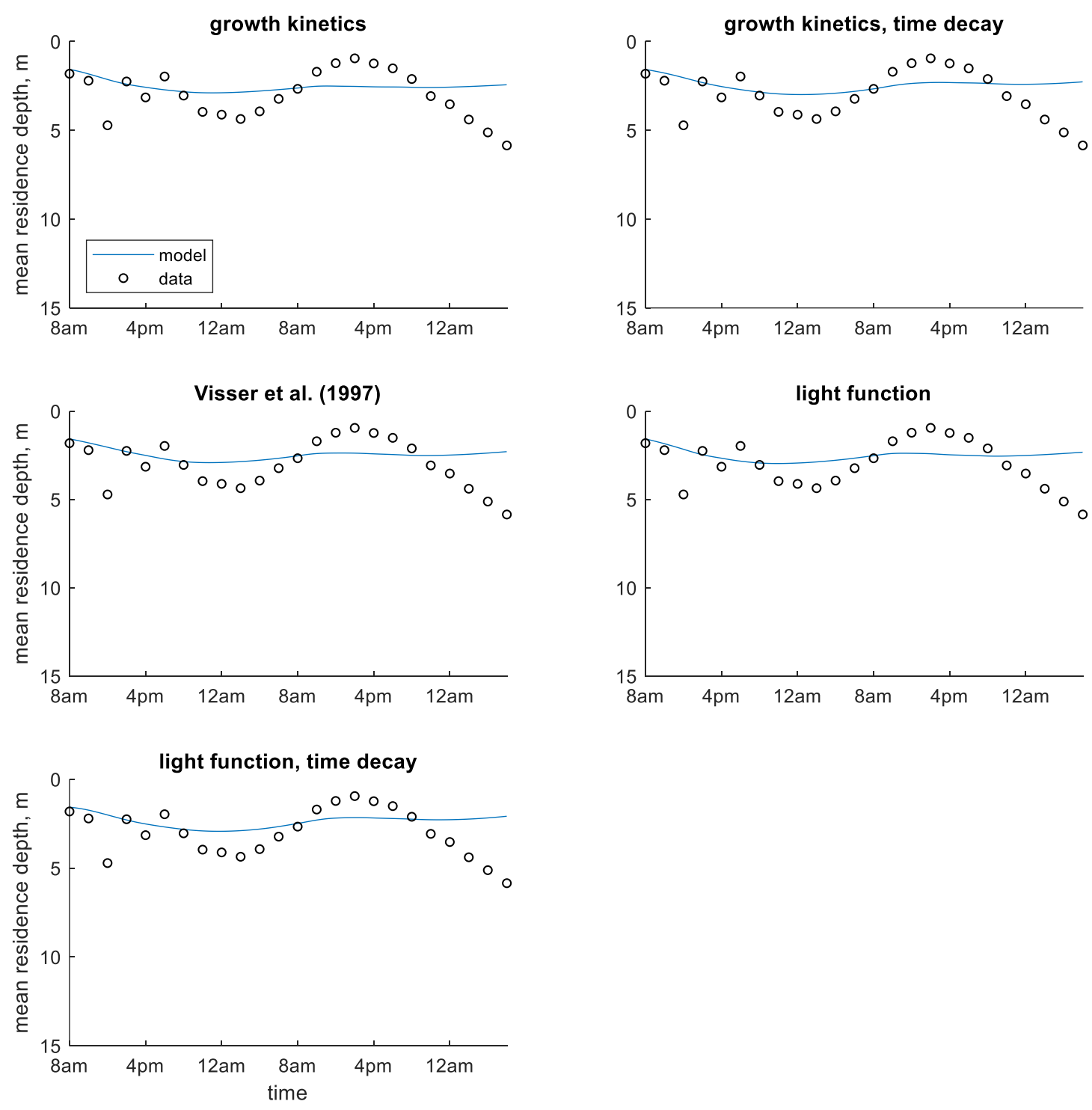

Figure 3-14 Time series of observed and predicted mean residence depth of chlorophyll a concentration in Shennong Stream open water site using dynamic velocity models (continuum) 

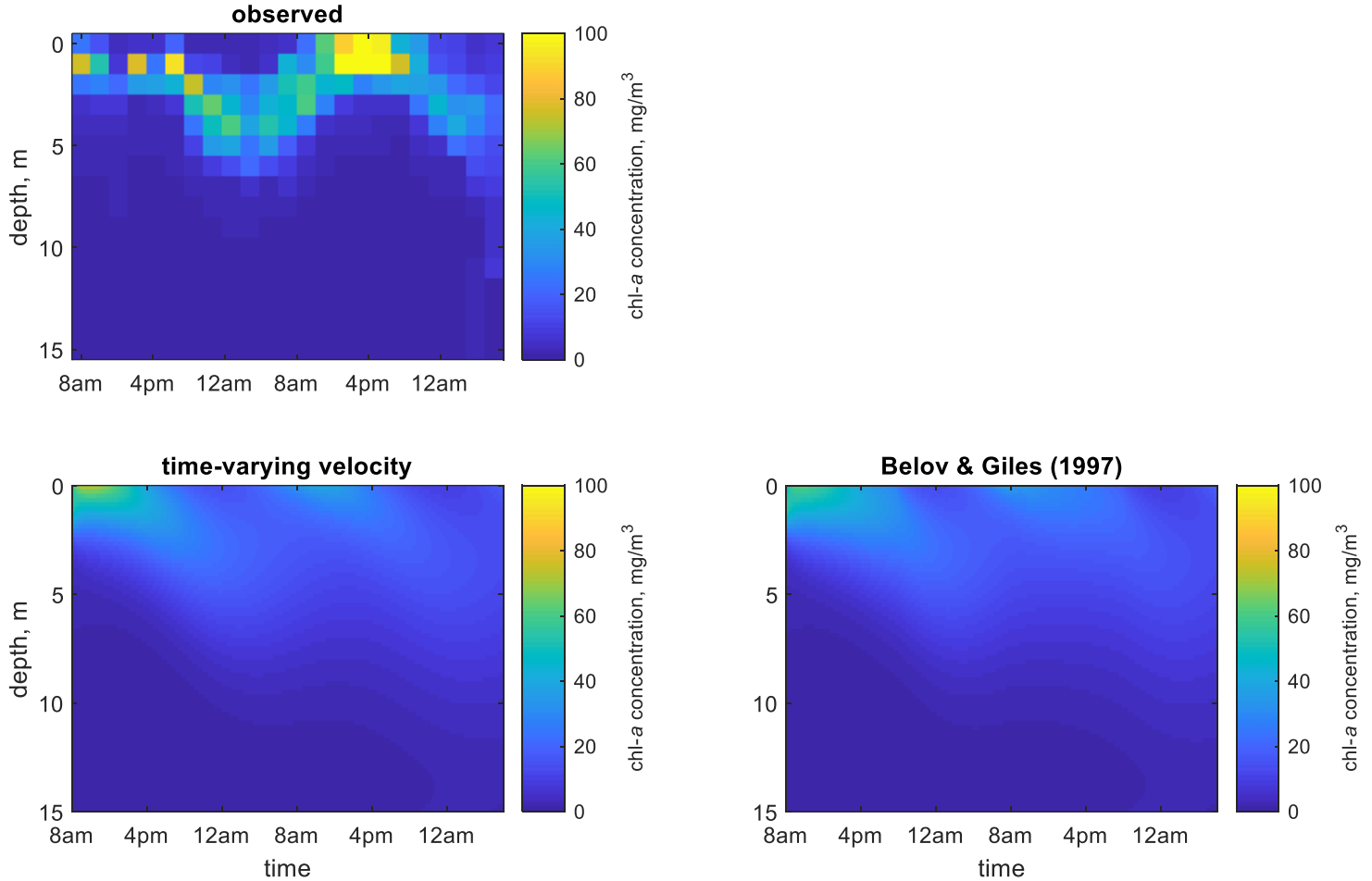

Figure 3-15 Time series of observed and predicted chlorophyll a concentration in Shennong Stream open water site using predefined velocity models (continuum) 

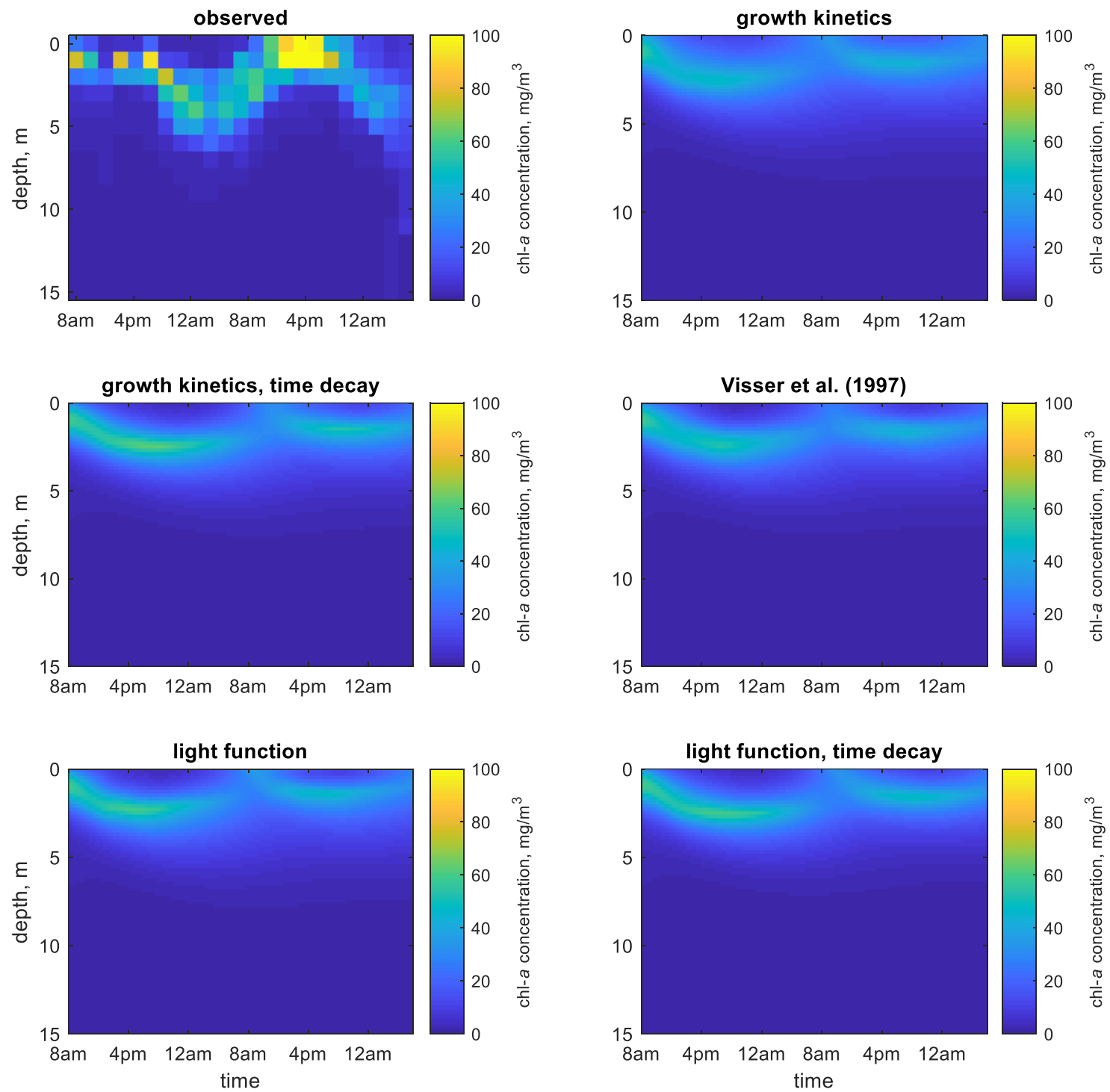

Figure 3-16 Time series of observed and predicted chlorophyll a concentration in Shennong Stream open water site using dynamic velocity models (continuum)

Results for the open water site using the particle-tracking framework did not show much variation across models. Dynamic velocity models resulted in lower error statistics than predefined velocity models, though only slightly (Table $3-5$ ). Results were mixed for chlorophyll a concentration error statistics; the growth kinetics model gave the lowest error, while the light function model gave the 
highest. Visual inspection of the MRD plots suggest that all models captured the general shape of the sinusoid curve, although on the second day the predefined velocity models start to predict a deeper MRD than observed while the dynamic velocity models predict too shallow of an MRD (Figure 3-17 \& Figure 3-18). The dynamic velocity models better reflect the level of chlorophyll a diffusion seen in the observed data than do the predefined velocity models (Figure 3-19 \& Figure 3-20).

Error statistics for MRD were again lower for dynamic velocity models in the particle-tracking framework compared to the continuum framework. However, the difference was not as much as seen in the models of the enclosure site, decreasing from 1.06-1.10 $\mathrm{m}$ to $0.96-0.99 \mathrm{~m}$ (Table 3-4 \& Table 3-5). Errors in chlorophyll a concentration predictions by dynamic velocity models increased from 7.19-7.73 $\mathrm{mg} / \mathrm{m}^{3}$ in the continuum framework to $8.50-8.67 \mathrm{mg} / \mathrm{m}^{3}$ in the particle-tracking framework. Differences between frameworks were smaller for both metrics in predefined velocity models.

Table 3-5 Error statistics from models of Shennong Stream open water site (particle-tracking)

\begin{tabular}{|l|r|l|r|}
\hline \multicolumn{2}{|c|}{ Mean Residence Depth } & \multicolumn{2}{c|}{$\begin{array}{c}\text { Chlorophyll a concentration } \\
\text { (profile average) }\end{array}$} \\
\hline \multicolumn{1}{|c|}{ Model } & AME, $\mathrm{m}$ & \multicolumn{1}{c|}{ Model } & AME, $\mathrm{mg} \mathrm{m}^{\mathbf{3}}$ \\
\hline Light function & 0.964 & Growth kinetics & 8.498 \\
\hline Growth kinetics & 0.986 & Time-varying velocity & 8.654 \\
\hline Visser et al. (1997) & 0.993 & Visser et al. (1997) & 8.670 \\
\hline Time-varying velocity & 1.097 & Belov \& Giles (1997) & 8.716 \\
\hline Belov \& Giles (1997) & 1.100 & Light function & 9.083 \\
\hline
\end{tabular}



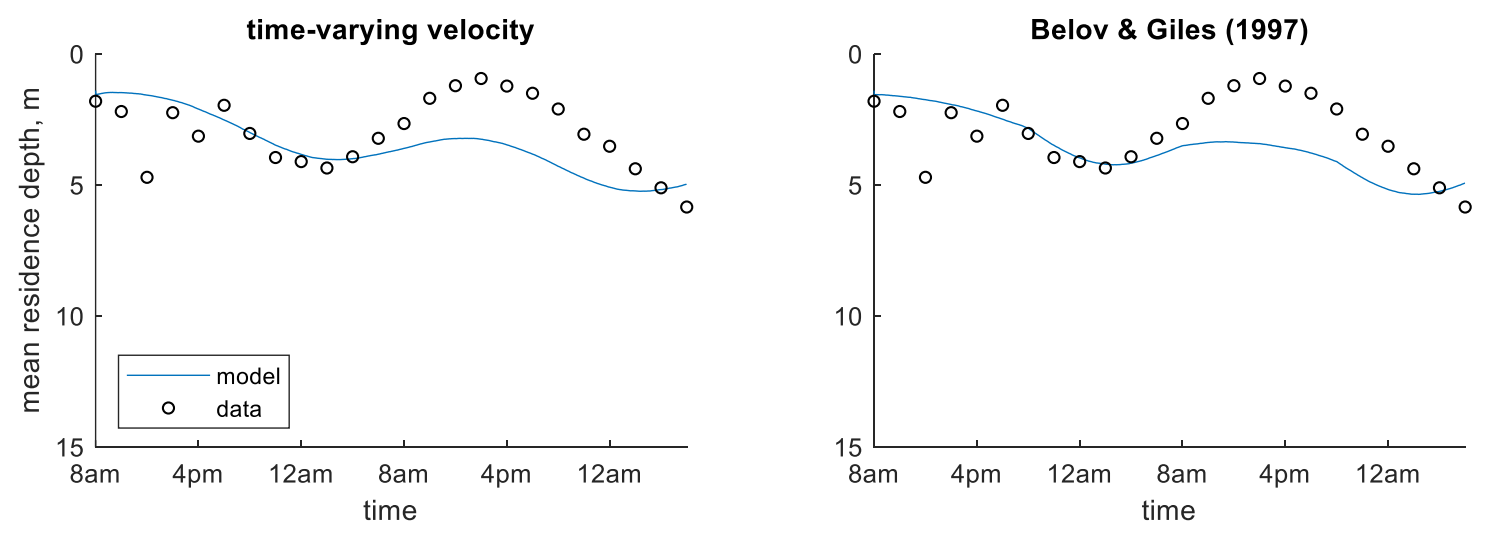

Figure 3-17 Time series of observed and predicted mean residence depth of chlorophyll a concentration in Shennong Stream open water site using predefined velocity models (particletracking)
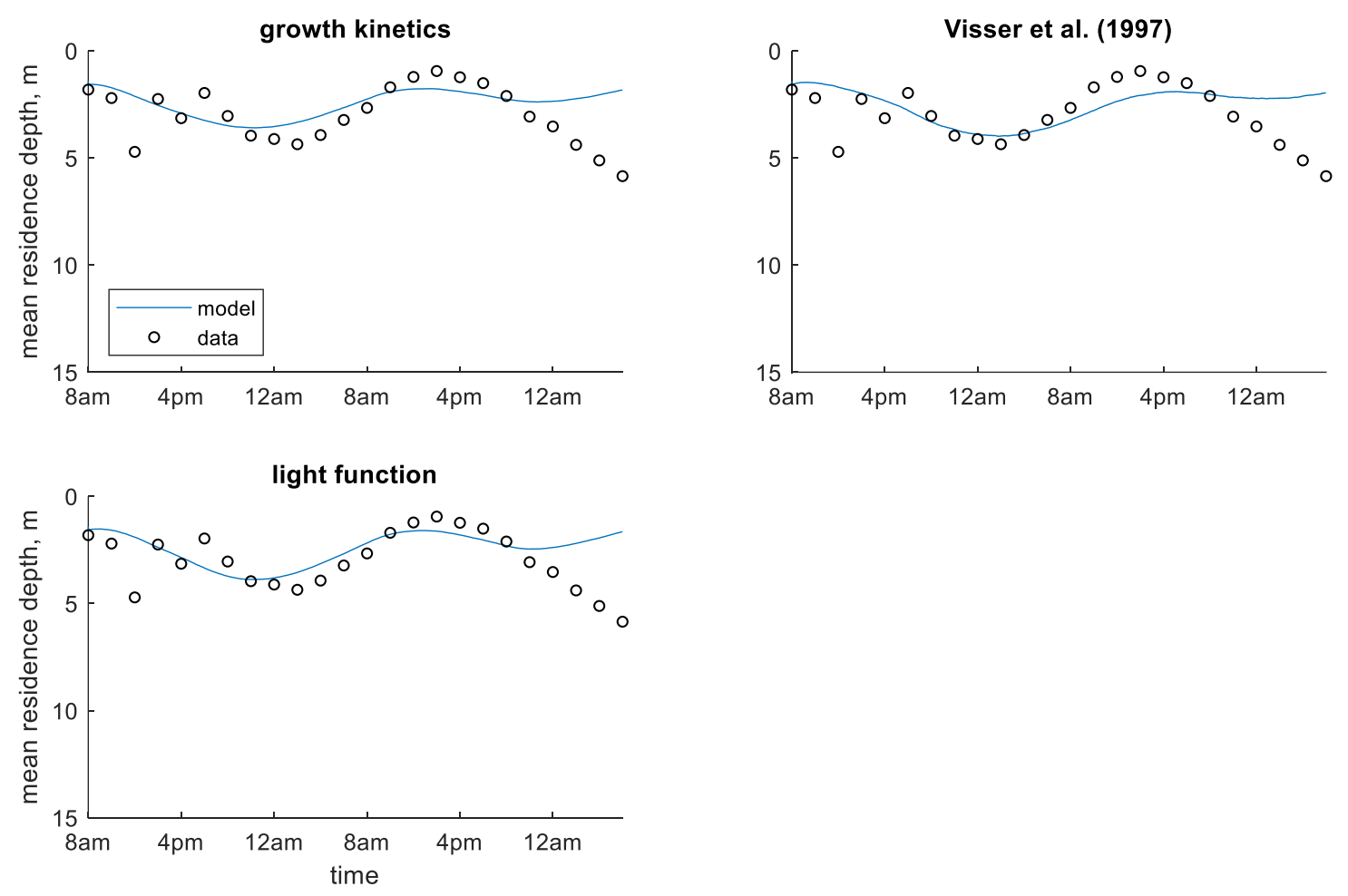

Figure 3-18 Time series of observed and predicted mean residence depth of chlorophyll a concentration in Shennong Stream open water site using dynamic velocity models (particle-tracking) 

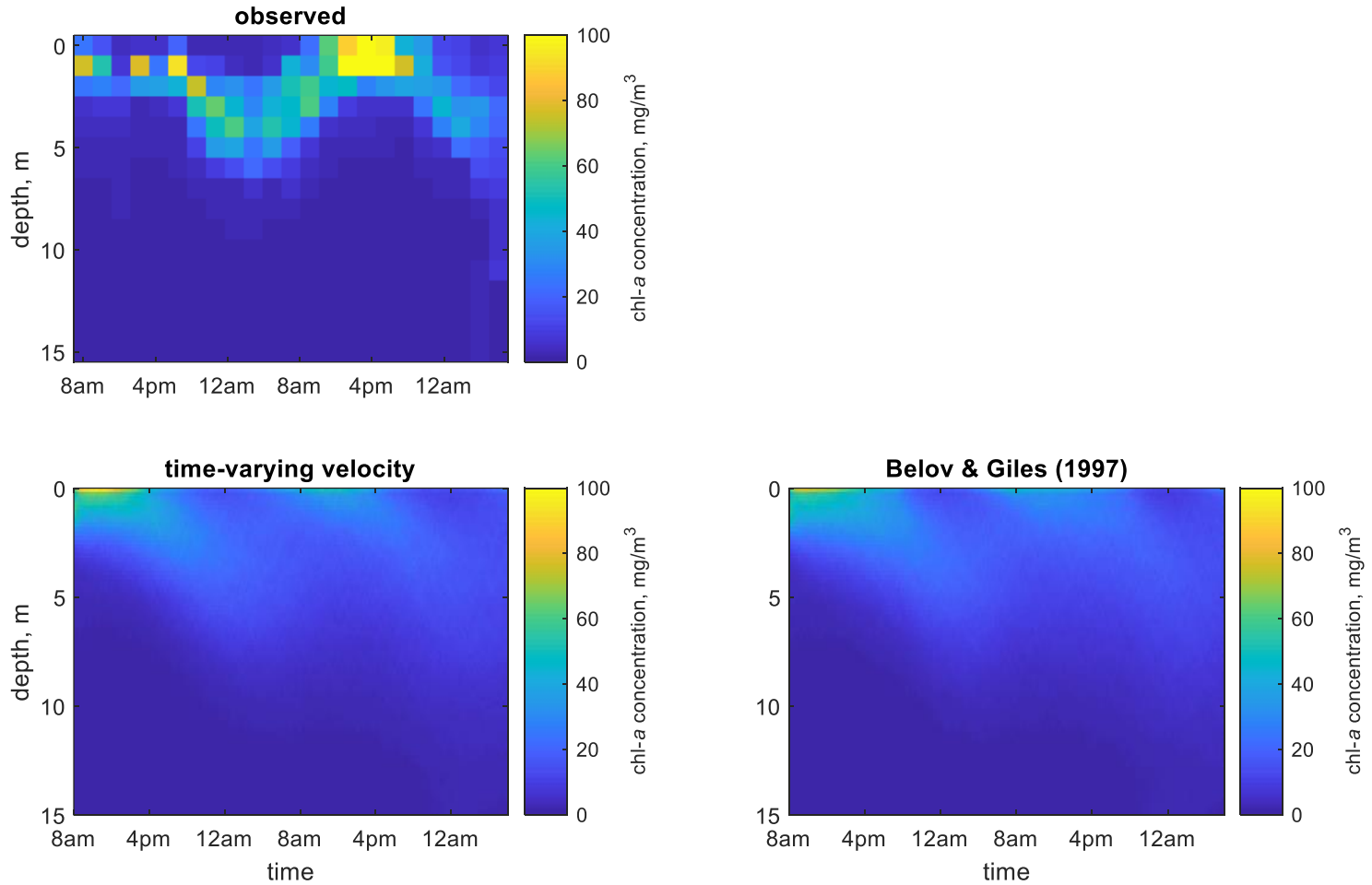

Figure 3-19 Time series of observed and predicted chlorophyll a concentration in Shennong Stream open water site using predefined velocity models (particle-tracking) 

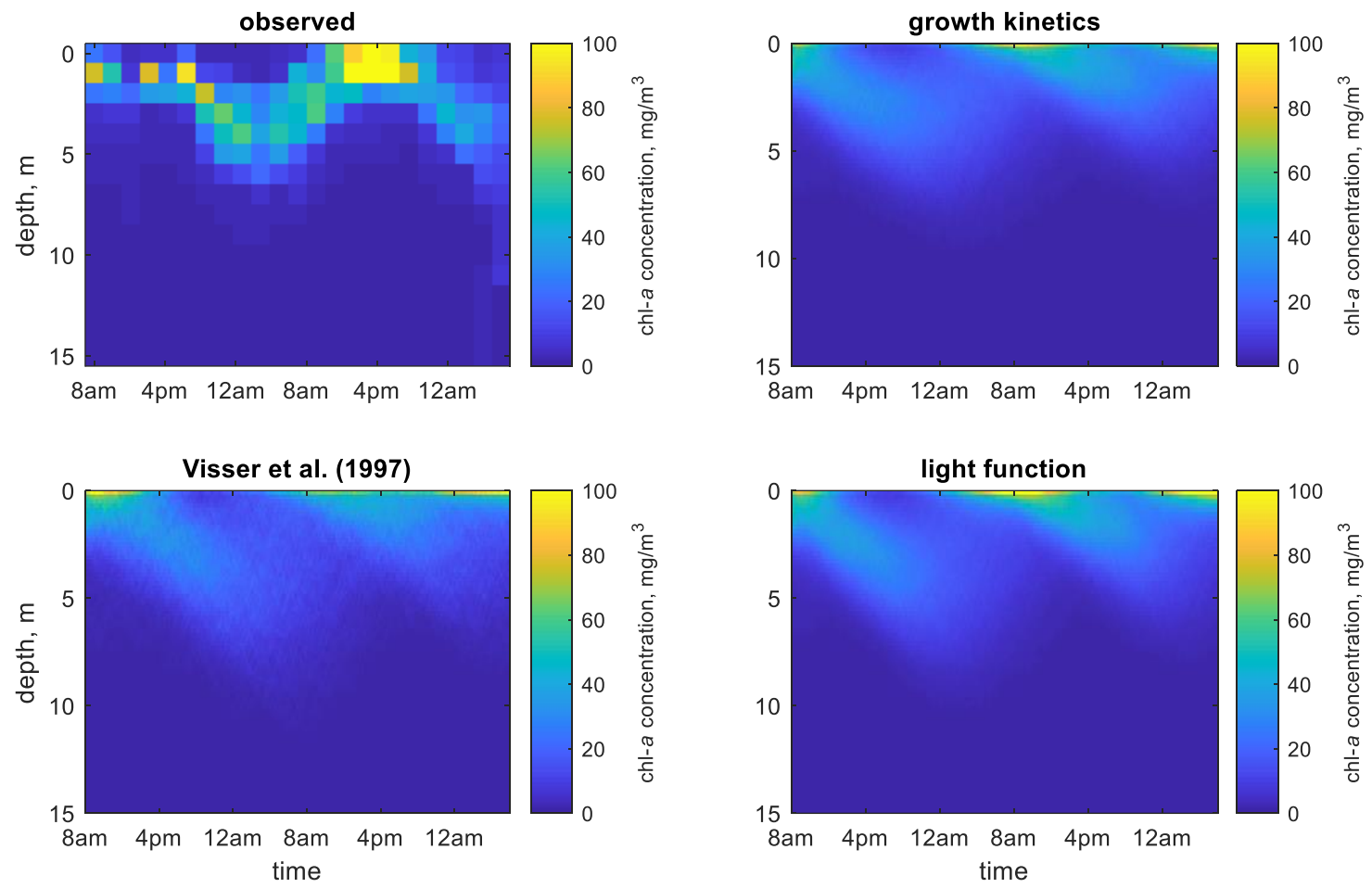

Figure 3-20 Time series of observed and predicted chlorophyll a concentration in Shennong Stream open water site using dynamic velocity models (particle-tracking)

\subsubsection{Xiangxi Bay}

Chlorophyll a contours from Xiangxi Bay show a steeper concentration gradient during the late morning and afternoon and more vertical diffusion in the middle of the night (Figure 3-3). The depth of maximum chlorophyll a concentration is near the surface on July 1 and July 7 and during the middle of the day on July 2 and 3. It moves down to approximately $5 \mathrm{~m}$ deep on the early morning on July 3 (Figure 3-3). MRD shows a sinusoidal pattern that reaches maximum depths in the middle of the night and shallow depths in the afternoon (Figure 3-4).

Within the continuum framework, the predefined velocity models gave better error statistics for the lower diffusion scenario. In plots of MRD, these models 
closely followed the pattern seen the field data (Figure 3-21), while the dynamic velocity models predicted a more shallow MRD than that seen in the data (Figure 3-22). The predefined velocity models also show more accurate predictions of depth of maximum chlorophyll a concentration when it is at its deepest between July 2 and 3 (Figure 3-23). Most of the dynamic velocity models do not correctly predict this, with the exception being the growth kinetics model (Table 3-6 \& Figure 3-24).

Table 3-6 Error statistics from models of Xiangxi Bay with $D_{z}=10^{-5} \mathrm{~m}^{2} \mathrm{~s}^{-1}$ (continuum)

\begin{tabular}{|c|c|c|c|}
\hline \multicolumn{2}{|c|}{ Mean Residence Depth } & \multicolumn{2}{|c|}{$\begin{array}{l}\text { Depth of Maximum Chlorophyll } a \\
\text { Concentration }\end{array}$} \\
\hline Model & $\mathrm{AME}, \mathrm{m}$ & Model & AME, $m$ \\
\hline Time-varying velocity & 0.351 & Time-varying velocity & 0.557 \\
\hline Belov \& Giles (1997) & 0.380 & Belov \& Giles (1997) & 0.561 \\
\hline Growth kinetics & 1.201 & Growth kinetics & 0.641 \\
\hline Visser et al. (1997) & 1.287 & Light function & 0.693 \\
\hline Growth kinetics with time decay & 1.299 & Light function with time decay & 0.725 \\
\hline Light function & 1.410 & Growth kinetics with time decay & 0.730 \\
\hline Light function with time decay & 1.650 & Visser et al. (1997) & 0.877 \\
\hline
\end{tabular}

For the higher diffusion scenario, there was not a clear distinction between the predefined and dynamic velocity models. The highest errors resulted from the growth kinetics and light function models without time decay (Table 3-7). Visually, the predefined velocity models seem to capture the shape of the MRD sinusoidal curve, but the dynamic velocity models predict the average depth more accurately (Figure 3-21 \& Figure 3-22). The dynamic models show more daily variation in depth of maximum chlorophyll a concentration for both scenarios but only the 
growth kinetics and light function models with time decay approximate the correct depth on the second day (Figure 3-23 \& Figure 3-24).

Table 3-7 Error statistics from models of Xiangxi Bay with $D_{z}=10^{-4} \mathrm{~m}^{2} \mathrm{~s}^{-1}$ (continuum)

\begin{tabular}{|l|r|l|r|}
\hline \multicolumn{2}{|c|}{ Mean Residence Depth } & \multicolumn{2}{c|}{$\begin{array}{c}\text { Depth of Maximum Chlorophyll } a \\
\text { Concentration }\end{array}$} \\
\hline \multicolumn{1}{|c|}{ Model } & AME, $\mathrm{m}$ & \multicolumn{1}{c|}{ Model } & AME, $\mathrm{m}$ \\
\hline Visser et al. (1997) & 0.374 & Growth kinetics with time decay & 0.667 \\
\hline Growth kinetics with time decay & 0.395 & Belov \& Giles (1997) & 0.680 \\
\hline Belov \& Giles (1997) & 0.409 & Light function with time decay & 0.687 \\
\hline Time-varying velocity & 0.422 & Time-varying velocity & 0.696 \\
\hline Light function with time decay & 0.426 & Growth kinetics & 0.744 \\
\hline Light function & 0.629 & Visser et al. (1997) & 0.754 \\
\hline Growth kinetics & 0.672 & Light function & 0.802 \\
\hline
\end{tabular}

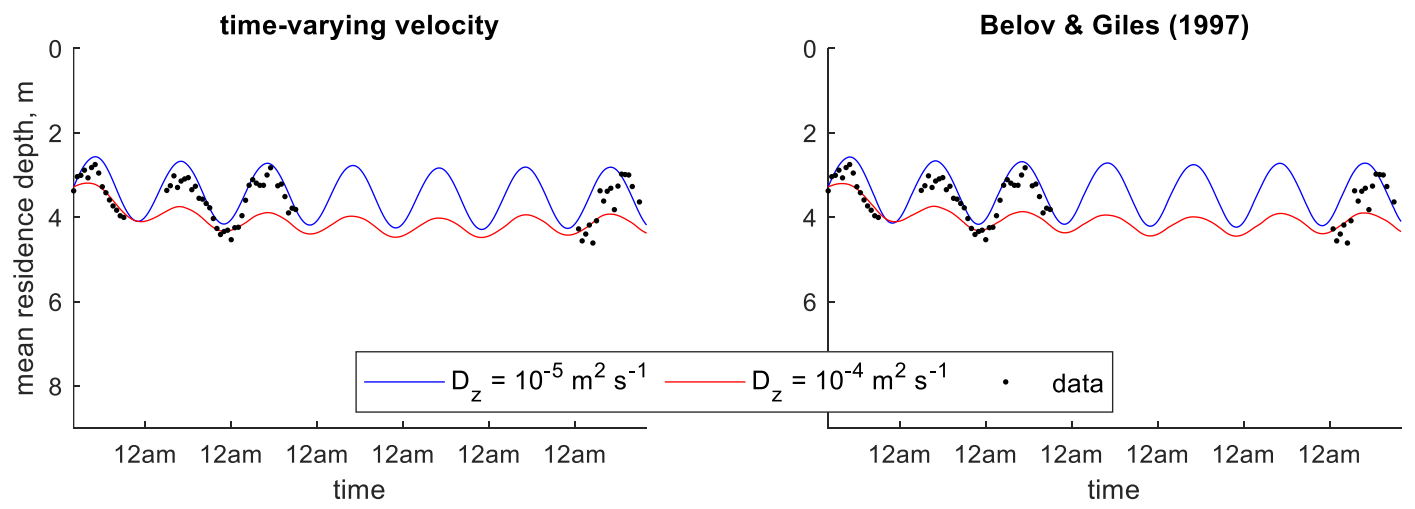

Figure 3-21 Time series of observed and predicted mean residence depth of chlorophyll a concentration in Xiangxi Bay using predefined velocity models (continuum) 

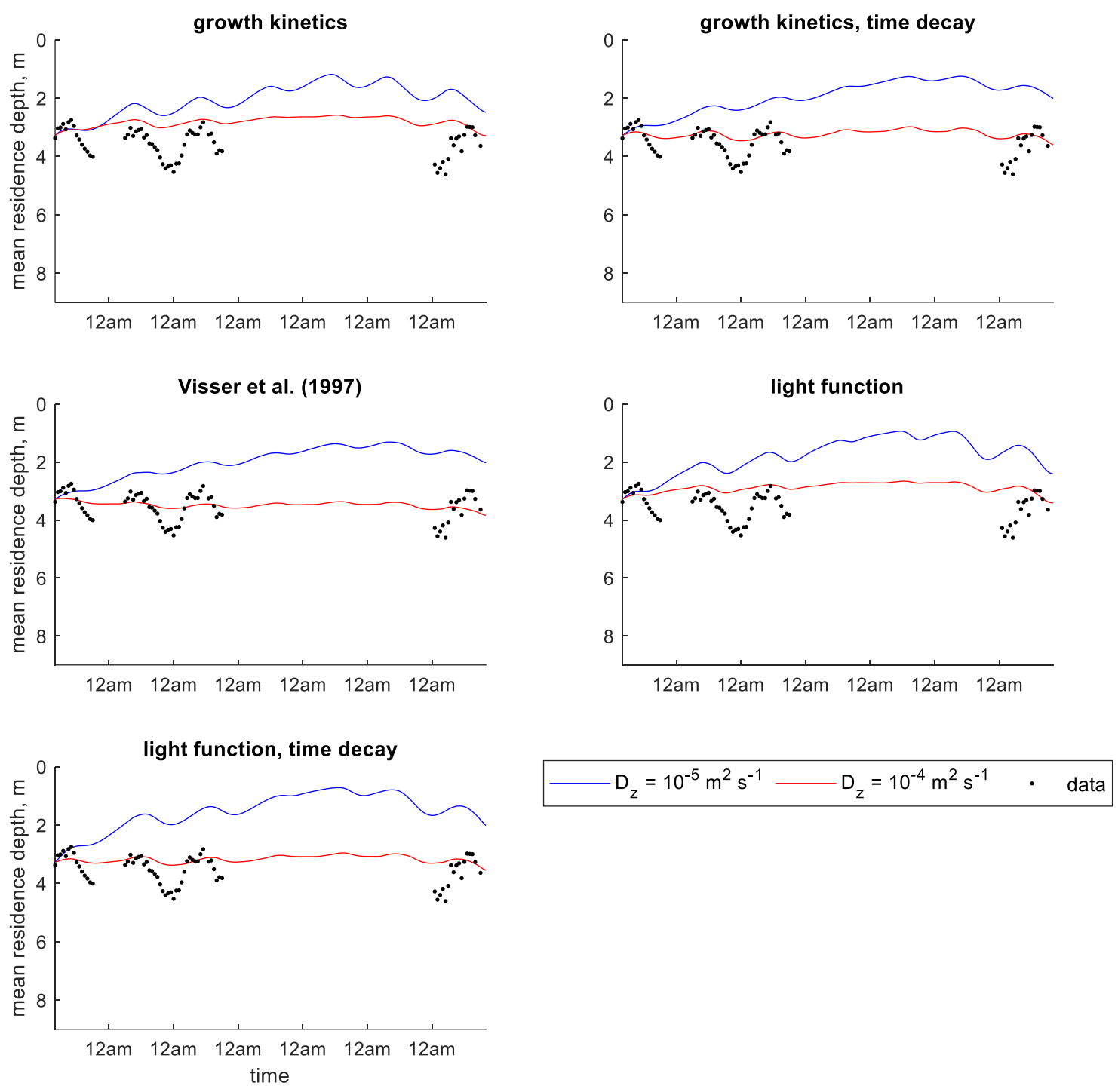

Figure 3-22 Time series of observed and predicted mean residence depth of chlorophyll a concentration in Xiangxi Bay using dynamic velocity models (continuum) 


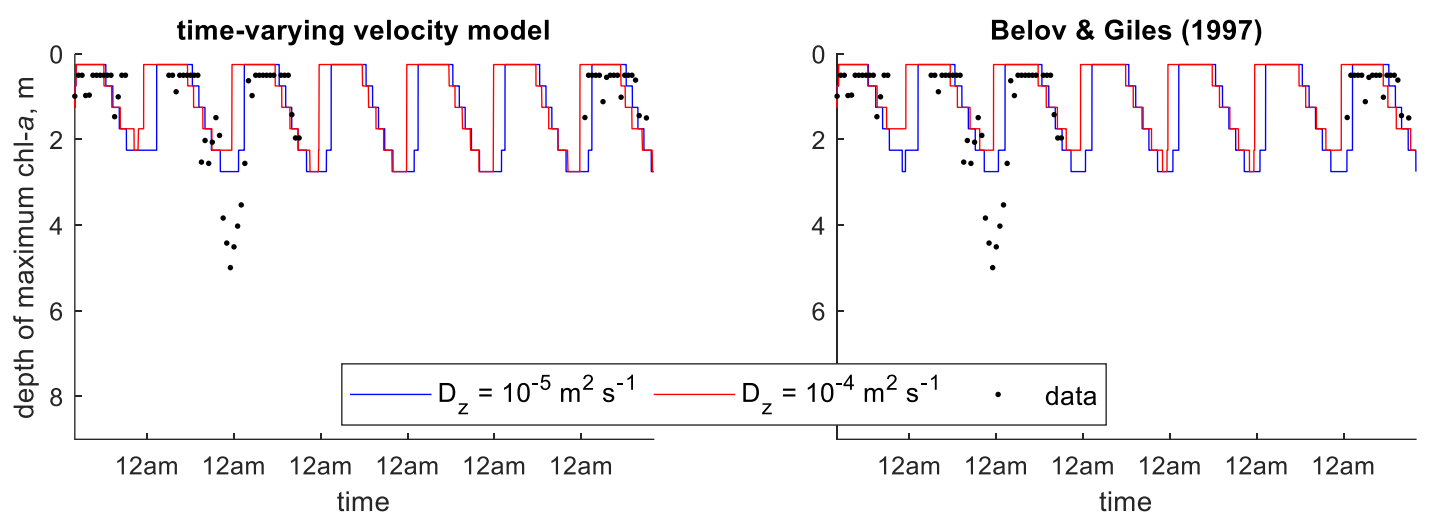

Figure 3-23 Time series of observed and predicted depth of maximum chlorophyll a concentration in Xiangxi Bay using predefined velocity models (continuum) 

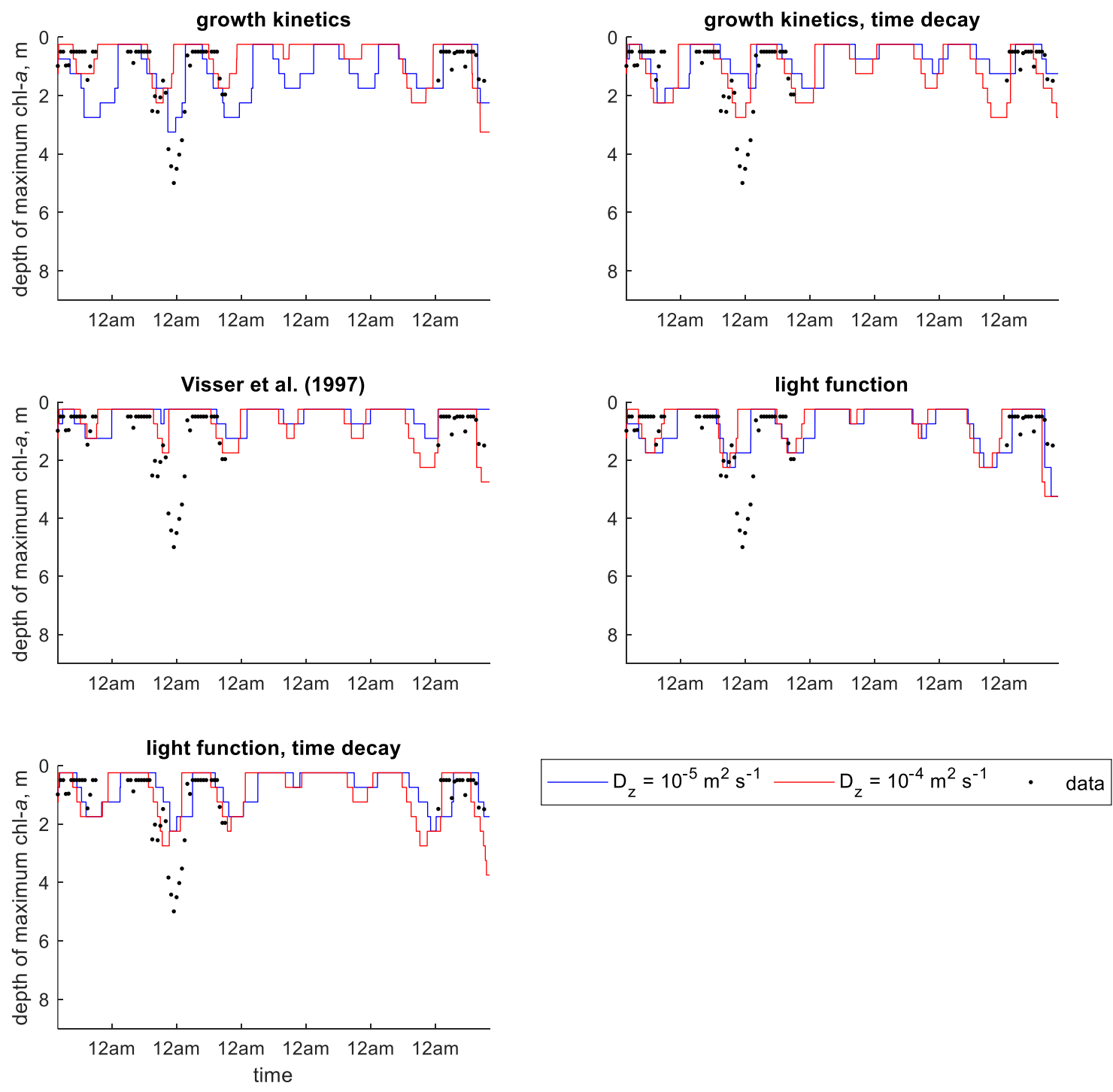

Figure 3-24 Time series of observed and predicted depth of maximum chlorophyll a concentration in Xiangxi Bay using dynamic velocity models (continuum)

Within the particle-tracking framework, the predefined velocity models gave the lowest error statistics for the lower value of $D_{z}$, although the growth kinetics model predicted MRD relatively well (Table 3-8). Plots of MRD show those models following the general pattern seen in the data throughout the study period (Figure 
3-25). Predictions from the dynamic velocity models predicted too great of an MRD amplitude or too shallow of a depth (Figure 3-26). The predefined velocity models in the particle-tracking framework also show the general pattern of depth of maximum chlorophyll a concentration (Figure 3-27), which was often predicted to be too deep in the dynamic velocity models (Figure 3-28).

Table 3-8 Error statistics from models of Xiangxi Bay with $D_{z}=10^{-5} \mathrm{~m}^{2} \mathrm{~s}^{-1}$ (particle-tracking)

\begin{tabular}{|l|r|l|r|}
\hline \multicolumn{2}{|c|}{ Mean Residence Depth } & \multicolumn{2}{c|}{$\begin{array}{c}\text { Depth of Maximum Chlorophyll } \boldsymbol{a} \\
\text { Concentration }\end{array}$} \\
\hline \multicolumn{1}{|c|}{ Model } & AME, $\mathrm{m}$ & \multicolumn{1}{c|}{ Model } & AME, $\mathrm{m}$ \\
\hline Time-varying velocity & 0.509 & Belov \& Giles (1997) & 0.554 \\
\hline Belov \& Giles (1997) & 0.585 & Time-varying velocity & 0.609 \\
\hline Growth kinetics & 0.595 & Growth kinetics & 1.598 \\
\hline Light function & 0.887 & Light function & 1.837 \\
\hline Visser et al. (1997) & 1.001 & Visser et al. (1997) & 2.800 \\
\hline
\end{tabular}

In the higher diffusion scenario, the dynamic velocity models resulted in better error statistics than the predefined velocity models for MRD (Table 3-9). Plots show that these models followed the general pattern of MRD, while the predefined velocity models predicted too small of an amplitude (Figure 3-25 \& Figure 3-26). While the error statistics show better results for depth of maximum chlorophyll a concentration in predefined velocity models, inspection of the plots suggests that the dynamic velocity models actually do better in this scenario. The predefined velocity models predict a depth of maximum chlorophyll a concentration that oscillates up and down at a high frequency in the evening, while the dynamic velocity models seem to follow the pattern seen in the data (Figure 3-27 \& Figure $3-28)$. 
Table 3-9 Error statistics from models of Xiangxi Bay with $D_{z}=10^{-4} \mathrm{~m}^{2} \mathrm{~s}^{-1}$ (particle-tracking)

\begin{tabular}{|l|r|l|r|}
\hline \multicolumn{2}{|c|}{ Mean Residence Depth } & \multicolumn{2}{c|}{$\begin{array}{c}\text { Depth of Maximum Chlorophyll } \boldsymbol{a} \\
\text { Concentration }\end{array}$} \\
\hline \multicolumn{1}{|c|}{ Model } & AME, $\mathrm{m}$ & \multicolumn{1}{c|}{ Model } & AME, $\mathrm{m}$ \\
\hline Light function & 0.371 & Belov \& Giles (1997) & 0.844 \\
\hline Visser et al. (1997) & 0.392 & Time-varying velocity & 0.846 \\
\hline Belov \& Giles (1997) & 0.413 & Growth kinetics & 1.373 \\
\hline Time-varying velocity & 0.415 & Light function & 1.454 \\
\hline Growth kinetics & 0.461 & Visser et al. (1997) & 1.598 \\
\hline
\end{tabular}

Similar to the Shennong Stream models, dynamic velocity models of the Xiangxi Bay study gave better error statistics of MRD in the particle-tracking framework than in the continuum framework for the lower diffusion scenario. In the continuum framework, AMEs ranged from $1.20 \mathrm{~m}$ to $1.65 \mathrm{~m}$ (Table 3-6), while in the particle-tracking framework they were between $0.59 \mathrm{~m}$ and $1.00 \mathrm{~m}$ (Table 3-8). The opposite was true for predefined velocity models, which had MRD AMEs of $0.35 \mathrm{~m}$ and $0.38 \mathrm{~m}$ in the continuum framework and $0.51 \mathrm{~m}$ and $0.58 \mathrm{~m}$ in the particletracking framework. Dynamic velocity models predicted depth of maximum chlorophyll a concentration better in the continuum framework than in the particletracking framework, with AMEs of 0.64-0.88 m versus 1.60-2.80 m. Little change was seen between frameworks for predefined velocity models in depth of maximum chlorophyll a concentration.

There was less of a distinct difference between continuum and particletracking frameworks in predictions of MRD for the higher diffusion scenario in Xiangxi Bay. AMEs did not change in predefined velocity models and were similar for dynamic velocity models (Table 3-7 \& Table 3-8). Errors were higher for depth 
of maximum chlorophyll a concentration in both predefined and dynamic velocity models in the particles-tracking framework compared to the continuum framework.

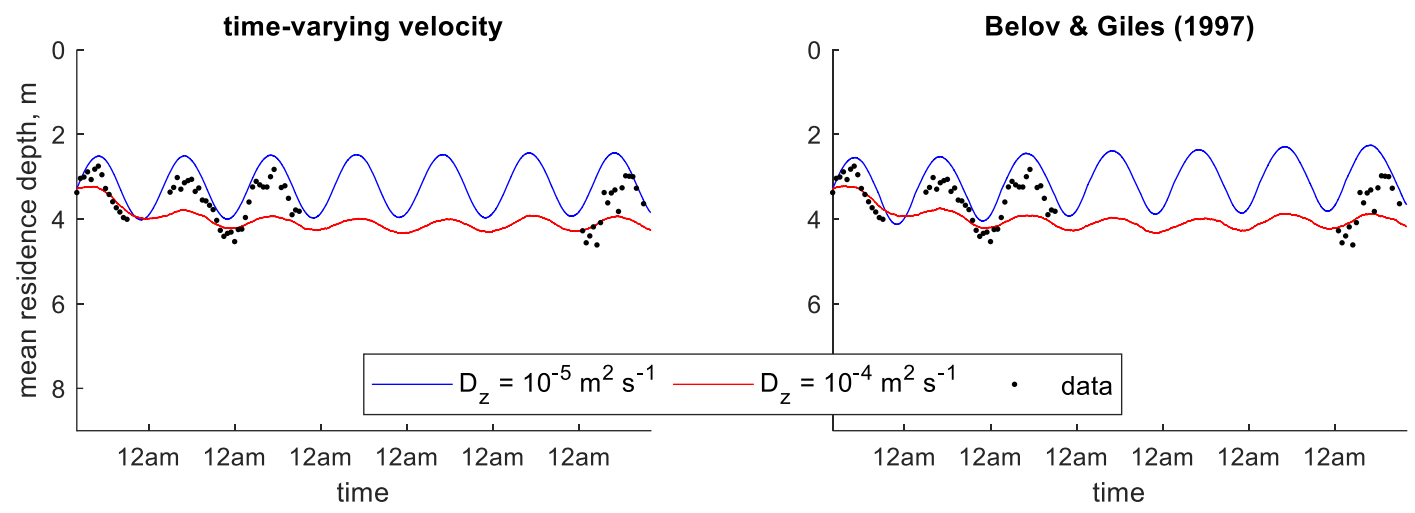

Figure 3-25 Time series of observed and predicted mean residence depth of chlorophyll a concentration in Xiangxi Bay using predefined velocity models (particle-tracking)
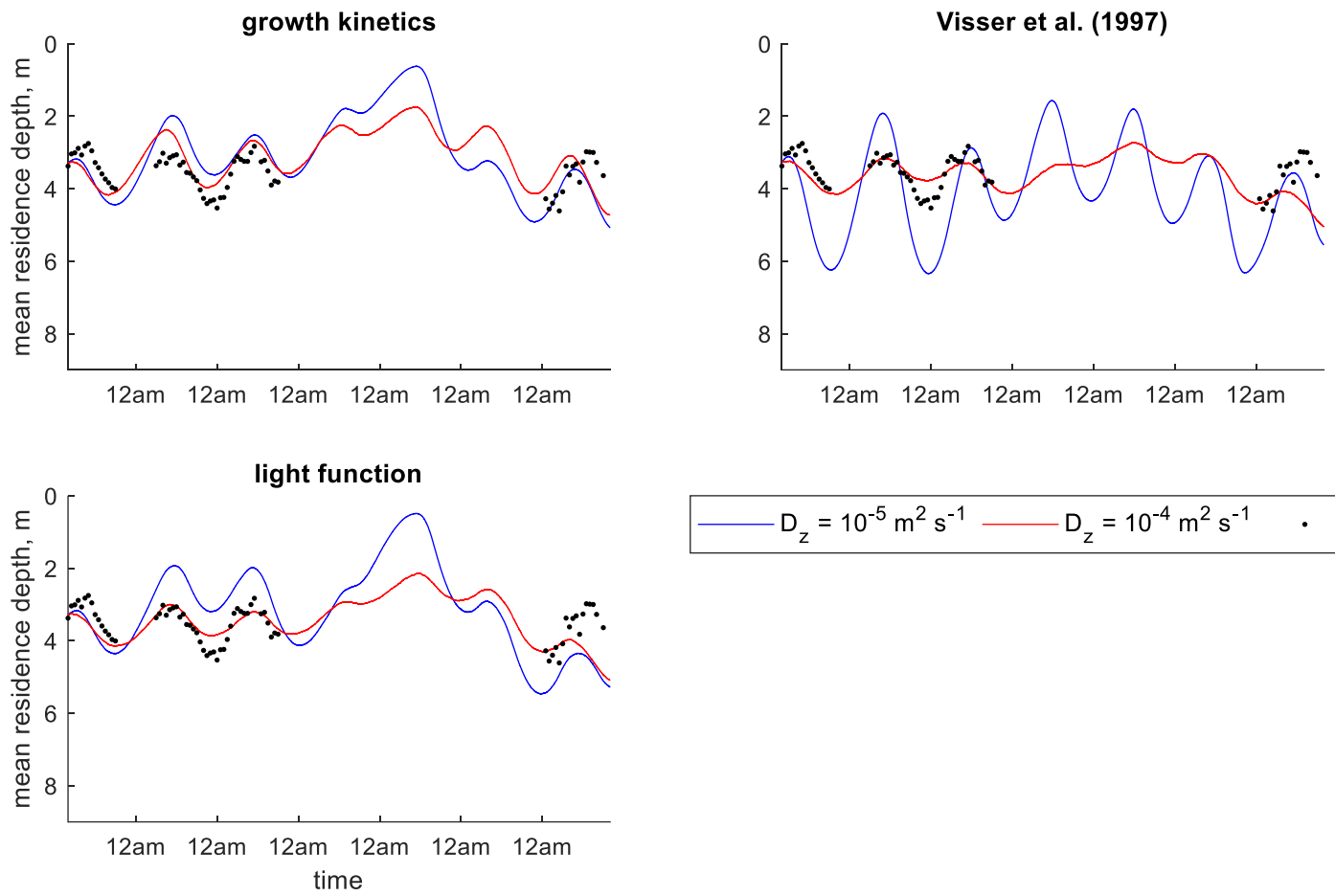

$\mathrm{D}_{\mathrm{z}}=10^{-5} \mathrm{~m}^{2} \mathrm{~s}^{-1}-\mathrm{D}_{\mathrm{z}}=10^{-4} \mathrm{~m}^{2} \mathrm{~s}^{-1} \cdot$ data

Figure 3-26 Time series of observed and predicted mean residence depth of chlorophyll a concentration in Xiangxi Bay using dynamic velocity models (particle-tracking) 


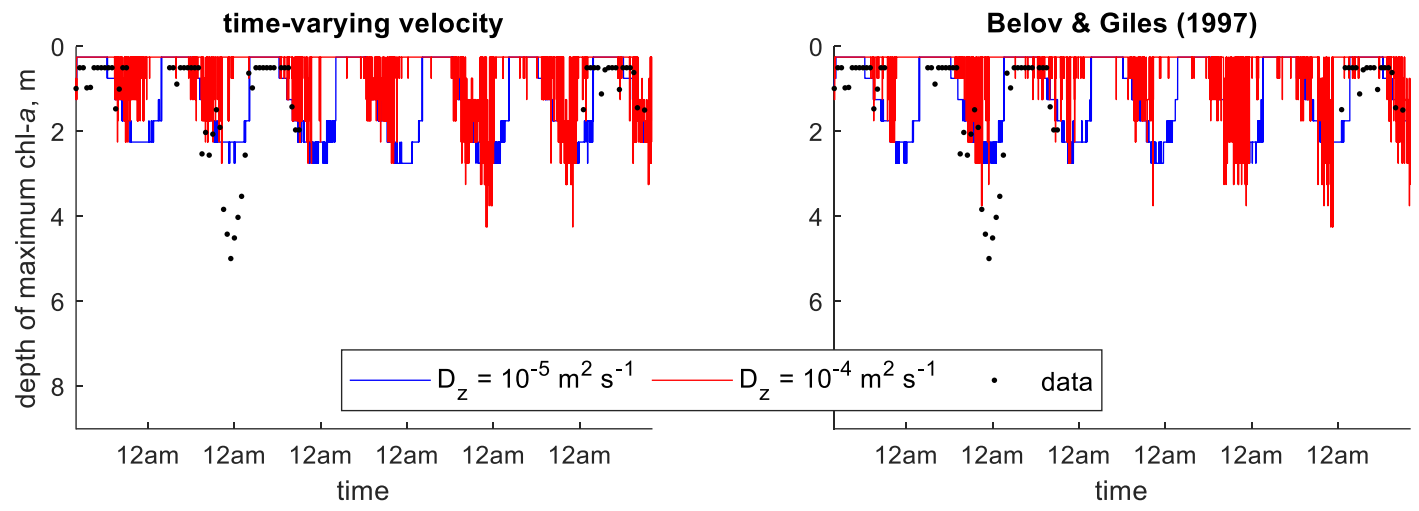

Figure 3-27 Time series of observed and predicted depth of maximum chlorophyll a concentration in Xiangxi Bay using predefined velocity models (particle-tracking)
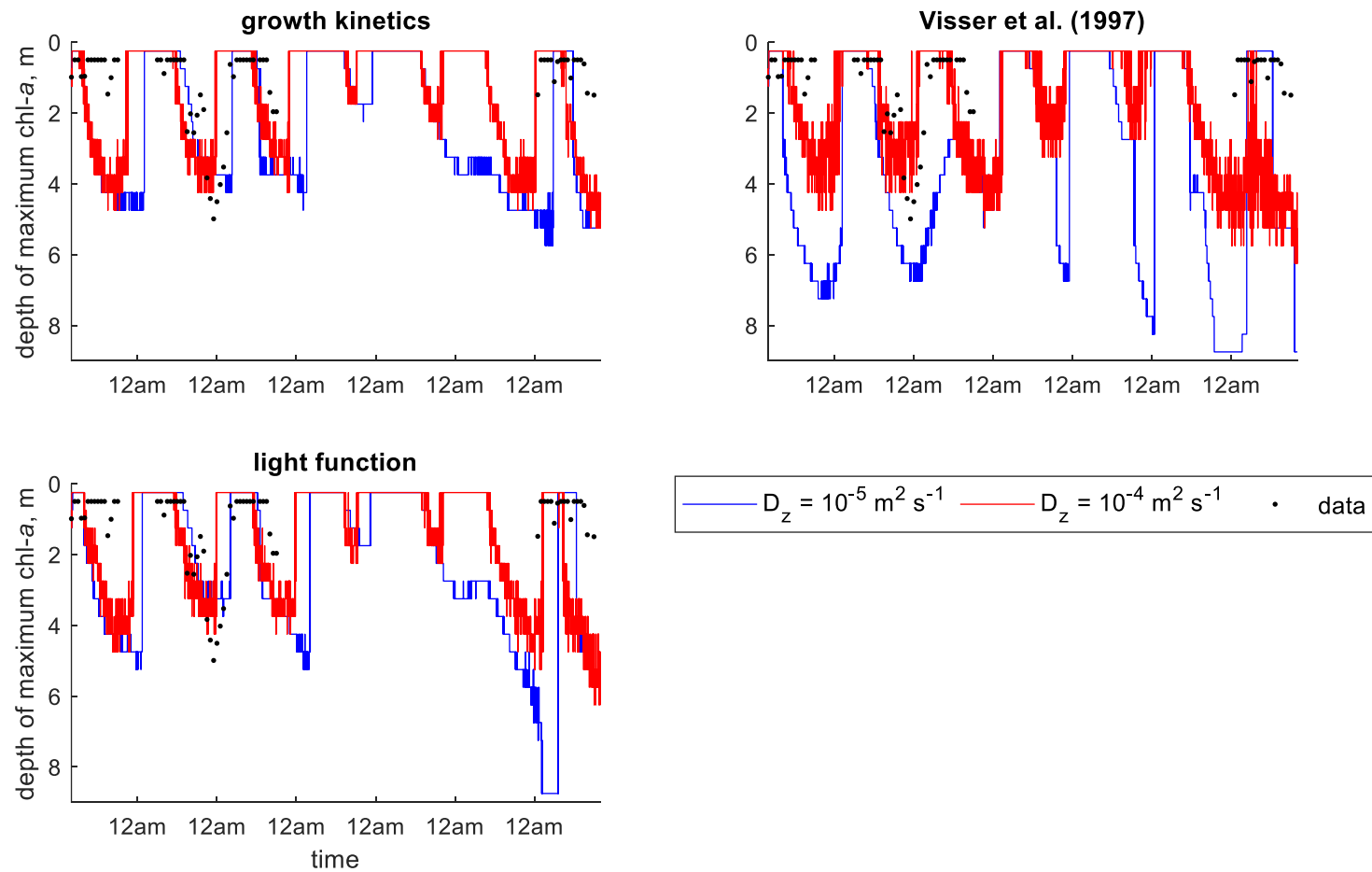

Figure 3-28 Time series of observed and predicted depth of maximum chlorophyll a concentration in Xiangxi Bay using dynamic velocity models (particle-tracking) 


\subsection{Discussion and Conclusions}

Both predefined velocity models and dynamic velocity models show benefits and drawbacks. The predefined velocity equations are simple to implement and often make good predictions of field data. However, they do not show daily variation due to changes in environmental inputs. The model of Belov and Giles (1997) can show the effect of changing water clarity, but not of changing solar irradiance. The dynamic velocity models are attractive because they represent the biological processes that are driving cyanobacteria vertical migration. The drawback is that they are more complicated to implement and include more parameters and tuning coefficients.

Typically, predefined velocity models performed better than dynamic velocity models at lower values of $D_{z}$. At higher values, predefined velocity models predicted concentrations lower than field values. This is likely due to all particles or model grid cells having the same velocity direction at the same time. The dynamic velocity models in the continuum framework generally made better predictions at higher values of $D_{z}$. In these models, there is a region below the water surface where downward velocities meet upward velocities. With less diffusion, a high concentration peak tends to develop in these areas. More diffusion spreads out this high concentration; however, these models often do not predict a deep enough excursion by cyanobacteria. 
A particle-tracking framework is better suited for dynamic velocity models, since these are based on tracking a specific colony though time and space as its density changes. This is not possible with a continuum framework, but the approximation made by solving for density in time and space was able to reproduce the expected pattern seen in field data. The addition of a time decay term generally helped the results in these cases. Predictions of MRD by dynamic velocity models were generally better in the particle-tracking framework than in continuum framework, while predictions of chlorophyll a concentration or depth of maximum chlorophyll a concentration were better in the continuum framework. This suggests that the particle-tracking framework better captures the overall shape of the concentration distribution when dynamic velocity equations are used. However, predictions of concentration at a specific depth are more erroneous. This could be due to the random-motion term in the particle-tracking framework and might be improved if more particles were used.

For the most part, models in particle-tracking and continuum frameworks following predefined velocity equations gave similar results. This is not surprising since the migration velocity is the same in both frameworks, unlike in the dynamic velocity models. The biggest difference seen here is that the models in the continuum framework became more spatially uniform from numerical diffusion. Models using a particle-tracking framework showed more random variation, although this is again dependent on the total number of particles used. 
In plots of observed and predicted MRD in the Shennong Stream open water site, none of the models captured the trajectory seen in the data at the end of the study period. Field data shows the MRD continuing to move downward from 4:00pm until sampling stopped at 6:00am, while the models predict it beginning to move upward just after midnight. The pattern seen in the data is unusual compared to data from the Shennong Stream enclosure site as well as Xiangxi Bay, where the MRD consistently begins to move upward around midnight. It is possible that the continued downward trajectory seen in the Shennong Stream open water data is caused by a hydrodynamic event and not cyanobacterial buoyancy regulation. This could explain why the vertical migration models presented here do not capture it, since water velocity was not included and vertical diffusion was assumed to be constant.

Some of the error in the model predictions could also be due to the assumption that the measured chlorophyll a concentration was entirely due to cyanobacteria. While Microcystis species were responsible for the majority of the phytoplankton concentration in both studies, other forms on non-migrating phytoplankton were present. This could lead to differences between the observed and predicted chlorophyll a metrics. 


\section{Chapter 4: Integration into CE-QUAL-W2}

\subsection{Selected Models}

The ultimate goal of this research is to add independent vertical motility to phyto- and zooplankton into the numerical hydrodynamic and water-quality model CE-QUAL-W2. After testing the various models in preliminary runs and on field studies, several models of cyanobacteria vertical migration were selected for incorporation into the continuum-concentration framework currently used to model plankton in CE-QUAL-W2. The models selected were the time-varying, predefined velocity model, the predefined velocity model based on the equations of Belov and Giles (1997), the model of Visser et al. (1997) and the light function model with time decay.

The two predefined velocity models were selected because they are simple while still being able to reproduce vertical migration patterns seen in field studies. They require the same inputs, with the addition of a calibration coefficient in the model based on Belov and Giles (1997).

The light function model with a time decay term was chosen because it generally gave good results in applications to field studies. It is preferable to the growth kinetics equation because it provides separate calibration coefficients from those used for modeling population growth kinetics. It is possible that adjustment to population growth rates could have undesirable effects in the buoyancy model if these two calculations rely on the same constants. Separating the rates of density 
change from the growth rates gives more freedom in calibrating the vertical migration model. The decay term was included because it almost always led to better model results in field studies than when it was not included.

The model of Visser et al. (1997) was chosen because it typically showed good results compared to the other dynamic velocity models in the continuum framework that did not include a time decay term. Including the decay term requires storing past densities and doing extra calculations, which can affect model computation time. The model of Visser et al. (1997) has the benefit of giving good results without storing past densities or making extra calculations. However, more variables are required by this model.

The vertical migration models were added to the Kinetic Rates Module in the Water Quality Subroutine of CE-QUAL-W2. Any number of distinct algal groups can be modeled in CE-QUAL-W2 (Cole and Wells, 2018). The addition of the vertical migration models gives the option of calculating a changing settling velocity for a particular algal group rather than using a constant velocity.

An input file was created, AlgeMigration.csv, that allows the model user to specify how many and which algal groups perform vertical migration, as well as the various parameters for each vertical migration model. In the predefined velocity models, users have the option to set intervals during which algae are allowed to migrate. Outside of those intervals, algae revert to their constant settling velocity (Table 4-1). All migration models allow users to set a depth limit for migration 
(DepthLim). Algae are not allowed to migrate past this depth; however, they could be carried past this depth by water currents (Table 4-1, Table 4-2, \& Table 4-3). Another user-defined variable applicable to all models is LossFraction, which is a number between 0 and 1 that determines how much of the algae in the bottommost layer will settle out of the water column and deposit onto the bed and how much will rise back into the water column. If this is set to 0 , all algae remain in the water column rather than settle out.

A feature was added to the Belov and Giles (1997) model to make it more suitable for deep waterbodies. The test case in the original study was $10 \mathrm{~m}$ deep, and the exponential depth term causes velocity to be largest at locations near the bed and small at locations far from the bed. In a deep waterbody, this would lead to algae having very small velocities unless they were close to the bed. To overcome this, the depth decay equation is only applied to the upper part of the waterbody. The depth of this region is determined as the depth at which light is $1 \%$ of the surface irradiance value if DepthCalc is turned on, or by the ExpLim parameter if DepthCalc is turned off. At depths below this region, migration velocity reduces to the time-varying velocity model.

For the light function model with time decay, the user selects a number of times steps to average past densities over, DecayTimeSteps (Table 4-2). For most model layers, this will mean that the density is a weighted average over the previous number of timesteps designated by the DecayTimeSteps. However, when water 
levels change, new model layers can be created. In this case, the weighted average could go back further in time since it is determined by the number of timesteps that a layer is active and not by a time interval.

Table 4-1 User-defined variables for migration Models 1 \& 2 (time-varying velocity and Belov \& Giles [1997])

\begin{tabular}{|c|c|c|c|}
\hline Variable & Description & $\begin{array}{c}\text { Time- } \\
\text { varying } \\
\text { velocity } \\
\text { (Model 1) }\end{array}$ & $\begin{array}{c}\text { Belov \& } \\
\text { Giles } \\
\text { (1997) } \\
\text { (Model 2) }\end{array}$ \\
\hline NumMigrateIntervals & $\begin{array}{l}\text { Number of intervals during which } \\
\text { phytoplankton are allowed to } \\
\text { migrate }\end{array}$ & - & - \\
\hline$J D A Y S t a r t$ & $\begin{array}{l}\text { Julian day of start of migration } \\
\text { interval }\end{array}$ & - & - \\
\hline JDAYEnd & $\begin{array}{l}\text { Julian day of end of migration } \\
\text { interval }\end{array}$ & - & - \\
\hline Amplitude, $m$ & Migration amplitude & $0.2-5$ & $0.2-5$ \\
\hline PhaseShift, rad & Phase offset & $\frac{\pi}{2}$ & $\frac{\pi}{2}$ \\
\hline CalibrationCoefficientExtinction & $\begin{array}{l}\text { Calibration coefficient for light } \\
\text { attenuation term }\end{array}$ & - & $0.05-0.20$ \\
\hline DepthCalc, 1 or 0 & $\begin{array}{l}\text { If on, model calculates maximum } \\
\text { depth to apply exponential decay } \\
\text { term for Model } 2\end{array}$ & - & - \\
\hline ExpDepth, $m$ & $\begin{array}{l}\text { If DepthCalc is off, sets maximum } \\
\text { depth in exponential decay term } \\
\text { for Model } 2\end{array}$ & - & - \\
\hline DepthLimOnOff, 1 or 0 & $\begin{array}{l}\text { If on, sets a maximum depth } \\
\text { beyond which phytoplankton } \\
\text { cannot migrate }\end{array}$ & - & - \\
\hline DepthLim, $m$ & $\begin{array}{l}\text { If DepthLimOnOff is on, sets } \\
\text { maximum migration depth }\end{array}$ & $25-50$ & $25-50$ \\
\hline LossFraction & $\begin{array}{l}\text { Fraction of phytoplankton that } \\
\text { settles out rather than resuspends }\end{array}$ & $0.05-0.10$ & $0.05-0.10$ \\
\hline
\end{tabular}


Table 4-2 User-defined variables for migration Model 3 (light function with time decay)

\begin{tabular}{|c|c|c|}
\hline Variable & Description & Value Range \\
\hline ColonyRadius, $m$ & Colony radius & $15-200 \times 10^{-6}$ \\
\hline MinColonyDensity, $\mathrm{kg} \mathrm{m}^{-3}$ & Minimum allowable colony density & $920-980$ \\
\hline MaxColonyDensity, $\mathrm{kg} \mathrm{m}^{-3}$ & Maximum allowable colony density & $1005-1085$ \\
\hline InitColonyDenSurf, $\mathrm{kg} \mathrm{m}^{-3}$ & Initial colony density at surface & $920-1085$ \\
\hline InitColonyDenBot, $\mathrm{kg} \mathrm{m}^{-3}$ & Initial colony density at bottom & $920-1085$ \\
\hline DecayTimeConstant, day ${ }^{-1}$ & Time decay constant for past density averaging & $1.5-8$ \\
\hline DecayTimeSteps & $\begin{array}{l}\text { Number of timesteps over which to average } \\
\text { past densities }\end{array}$ & $100-500$ \\
\hline CoeffDenIncr, $\mathrm{kg} \mathrm{m}^{-3} \mathrm{~s}^{-1}$ & $\begin{array}{l}\text { Coefficient of density increase for density } \\
\text { change equation }\end{array}$ & $0.06-0.03$ \\
\hline CoeffDenDecr, $\mathrm{kg} \mathrm{m}^{-3} \mathrm{~s}^{-1}$ & $\begin{array}{l}\text { Coefficient of density decrease for density } \\
\text { change equation }\end{array}$ & $0.001-0.006$ \\
\hline DepthLimOnOff, 1 or 0 & $\begin{array}{l}\text { If on, sets a maximum depth beyond which } \\
\text { phytoplankton cannot migrate }\end{array}$ & \\
\hline DepthLim, $m$ & $\begin{array}{l}\text { If DepthLimOnOff is on, sets maximum } \\
\text { migration depth }\end{array}$ & $25-50$ \\
\hline LossFraction & $\begin{array}{l}\text { Fraction of phytoplankton that settles out } \\
\text { rather than resuspends }\end{array}$ & $0.05-0.10$ \\
\hline
\end{tabular}

The issue of adding a new layer is relevant when using the two dynamic velocity approaches, since they both rely on change in density for velocity calculations. If a model grid cell becomes active, e.g. a new surface layer is added that was not there during the previous timestep, the previous density is assumed to be the same as the previous density in the cell directly below.

In the model based on equations from Visser et al. (1997), the coefficients for the equations predicting density increase and decease as well as the compensation irradiance are all user defined variables (Table 4-3). However, these were not calibrated in the preliminary models and field data applications here and the values given are the values from the original study. 
Table 4-3 User-defined variables for migration Model 4 (Visser et al. [1997])

\begin{tabular}{|c|c|c|}
\hline Variable & Description & Value/Range \\
\hline ColonyRadius, $m$ & Colony radius & $15-200 \times 10^{-6}$ \\
\hline MinColonyDensity, $\mathrm{kg} \mathrm{m}^{-3}$ & Minimum allowable colony density & $920-980$ \\
\hline MaxColonyDensity, $\mathrm{kg} \mathrm{m}^{-3}$ & Maximum allowable colony density & $1005-1085$ \\
\hline InitColonyDenSurf, $\mathrm{kg} \mathrm{m}^{-3}$ & Initial colony density at surface & $920-1085$ \\
\hline InitColonyDenBot, $\mathrm{kg} \mathrm{m}^{-3}$ & Initial colony density at bottom & $920-1085$ \\
\hline CompIrad, $W m^{-2}$ & Compensation irradiance for density increase & 5.45 \\
\hline CoeffDenIncr $_{1}, s^{2} m^{-3}$ & Coefficient 1 of density increase equation & $5.33 \times 10^{-5}$ \\
\hline CoeffDenIncr $\mathrm{kg} \mathrm{m}^{-3} \mathrm{~s}^{-1}$ & Coefficient 2 of density increase equation & $-2.75 \times 10^{-4}$ \\
\hline CoeffDenDecr ${ }_{1}, s^{-1}$ & Coefficient 1 of density decrease equations & $1.58 \times 10^{-5}$ \\
\hline CoeffDenDecr ${ }_{2}, \mathrm{~kg} \mathrm{~m}^{-3} \mathrm{~s}^{-1}$ & Coefficient 2 of density decrease equations & $1.64 \times 10^{-2}$ \\
\hline MinDenDecrSurf, $\mathrm{kg} \mathrm{m}^{-3}$ & $\begin{array}{l}\text { Minimum allowable density at the surface for use } \\
\text { in density decrease equation }\end{array}$ & $980-1080$ \\
\hline MinDenDecrBot, $\mathrm{kg} \mathrm{m}^{-3}$ & $\begin{array}{l}\text { Minimum allowable density at the bottom for use } \\
\text { in density decrease equation }\end{array}$ & $975-980$ \\
\hline DenCorrection, $\mathrm{kg} \mathrm{m}^{-3}$ & Correction factor for density decrease equation & 65 \\
\hline DepthLimOnOff, 1 or 0 & $\begin{array}{l}\text { If on, sets a maximum depth beyond which } \\
\text { phytoplankton cannot migrate }\end{array}$ & - \\
\hline DepthLim, $m$ & $\begin{array}{l}\text { If DepthLimOnOffis on, sets maximum migration } \\
\text { depth }\end{array}$ & $25-50$ \\
\hline LossFraction & $\begin{array}{l}\text { Fraction of phytoplankton that settle out rather } \\
\text { than resuspends }\end{array}$ & $0.05-0.10$ \\
\hline
\end{tabular}

\subsection{Test Case: Dexter Reservoir}

The new cyanobacteria vertical migration additions to CE-QUAL-W2 were tested on an existing model of Dexter Reservoir. Dexter Reservoir is located on the Middle Fork Willamette River upstream of Eugene, Oregon. It is immediately downstream of a larger reservoir, Lookout Point, and regulates the fluctuations in water level due to hydroelectricity production in Lookout Point Dam. The model was developed by researchers at Portland State University and is currently calibrated for June 2016- November 2017 (Berger et al., 2018). Dexter Reservoir 
has a history of HABs due to cyanobacteria. For these reasons, Dexter Reservoir was chosen as a test case for the vertical migration models added to CE-QUAL-W2.

\subsubsection{Model Setup}

All four migration models were tested on the 2016-2017 model of Dexter Reservoir. The model includes three algal groups. Vertical migration models were applied to Algal Group 2, which was already calibrated to represent cyanobacteria. Predefined velocity models were run with one migration interval for the entire simulation (Table 4-4). Other variables used in the migration models were set to values similar to those used in the field data test cases (Table 4-4, Table 4-5, \& Table 4-6). A control model was also run in which Algal Group 2 was assigned a negative (upward) settling velocity to represent floating cyanobacteria. 
Table 4-4 Variables used in application of CE-QUAL-W2 algae migration Models 1 \& 2 to Dexter Reservoir model

\begin{tabular}{|c|c|c|c|}
\hline Variable & Description & $\begin{array}{c}\text { Time- } \\
\text { varying } \\
\text { velocity } \\
\text { (Model 1) }\end{array}$ & $\begin{array}{c}\text { Belov \& } \\
\text { Giles } \\
\text { (1997) } \\
\text { (Model 2) }\end{array}$ \\
\hline NumMigrateIntervals & $\begin{array}{l}\text { Number of intervals during which } \\
\text { phytoplankton are allowed to } \\
\text { migrate }\end{array}$ & 1 & 1 \\
\hline JDAYStart & $\begin{array}{l}\text { Julian day of start of migration } \\
\text { interval }\end{array}$ & 167.5 & 167.5 \\
\hline JDAYEnd & $\begin{array}{l}\text { Julian day of end of migration } \\
\text { interval }\end{array}$ & 678 & 678 \\
\hline Amplitude, $m$ & Migration amplitude & 0.6 & 0.75 \\
\hline PhaseShift, rad & Phase offset & $\frac{\pi}{2}$ & $\frac{\pi}{2}$ \\
\hline CalibrationCoefficientExtinction & $\begin{array}{l}\text { Calibration coefficient for light } \\
\text { attenuation term }\end{array}$ & - & 0.05 \\
\hline DepthCalc, 1 or 0 & $\begin{array}{l}\text { If on, model calculates maximum } \\
\text { depth to apply exponential decay } \\
\text { term for Model } 2\end{array}$ & - & 1 \\
\hline ExpDepth, m & $\begin{array}{l}\text { If DepthCalc is off, sets maximum } \\
\text { depth in exponential decay term } \\
\text { for Model } 2\end{array}$ & - & - \\
\hline DepthLimOnOff, 1 or 0 & $\begin{array}{l}\text { If on, sets a maximum depth } \\
\text { beyond which phytoplankton } \\
\text { cannot migrate }\end{array}$ & 0 & 0 \\
\hline DepthLim, $m$ & $\begin{array}{l}\text { If DepthLimOnOffis on, sets } \\
\text { maximum migration depth }\end{array}$ & - & - \\
\hline LossFraction & $\begin{array}{l}\text { Fraction of phytoplankton that } \\
\text { settles out rather than resuspends }\end{array}$ & 0.05 & 0.05 \\
\hline
\end{tabular}


Table 4-5 Variables used in application of CE-QUAL-W2 algae migration Model 3 to Dexter Reservoir model

\begin{tabular}{|l|l|c|}
\hline \multicolumn{1}{|c|}{ Variable } & \multicolumn{1}{|c|}{ Description } & Value/Range \\
\hline ColonyRadius, $\mathrm{m}$ & Colony radius & $15 \times 10^{-6}$ \\
\hline MinColonyDensity, $\mathrm{kg} \mathrm{m}^{-3}$ & Minimum allowable colony density & 920 \\
\hline MaxColonyDensity, $\mathrm{kg} \mathrm{m}^{-3}$ & Maximum allowable colony density & 1060 \\
\hline InitColonyDenSurf, $\mathrm{kg} \mathrm{m}^{-3}$ & Initial colony density at surface & 980 \\
\hline InitColonyDenBot, $\mathrm{kg} \mathrm{m}^{-3}$ & Initial colony density at bottom & 920 \\
\hline DecayTimeConstant, $\mathrm{day}^{-1}$ & Time decay constant for past density averaging & 7 \\
\hline DecayTimeSteps & $\begin{array}{l}\text { Number of timesteps over which to average past } \\
\text { densities }\end{array}$ & 100 \\
\hline CoeffDenIncr, $\mathrm{kg} \mathrm{m}^{-3} \mathrm{~s}^{-1}$ & $\begin{array}{l}\text { Coefficient of density increase for density change } \\
\text { equation }\end{array}$ & 0.0115 \\
\hline CoeffDenDecr, $\mathrm{kg} \mathrm{m}^{-3} \mathrm{~s}^{-1}$ & $\begin{array}{l}\text { Coefficient of density decrease for density change } \\
\text { equation }\end{array}$ & 0.005 \\
\hline DepthLimOnOff, 1 or 0 & $\begin{array}{l}\text { If on, sets a maximum depth beyond which } \\
\text { phytoplankton cannot migrate }\end{array}$ & 0FF \\
\hline DepthLim, $m$ & $\begin{array}{l}\text { If DepthLimOnOffis turned on, maximum } \\
\text { migration depth }\end{array}$ & $\begin{array}{l}\text { Fraction of phytoplankton that settles out rather } \\
\text { than resuspends }\end{array}$ \\
\hline LossFraction & 0.05 \\
\hline
\end{tabular}


Table 4-6 Variables used in application of CE-QUAL-W2 algae migration Model 4 to Dexter Reservoir model

\begin{tabular}{|c|c|c|}
\hline Variable & Description & Value/Range \\
\hline ColonyRadius, $m$ & Colony radius & $20 \times 10^{-6}$ \\
\hline MinColonyDensity, $\mathrm{kg} \mathrm{m}^{-3}$ & Minimum allowable colony density & 920 \\
\hline MaxColonyDensity, $\mathrm{kg} \mathrm{m}^{-3}$ & Maximum allowable colony density & 1050 \\
\hline InitColonyDenSurf, $\mathrm{kg} \mathrm{m}^{-3}$ & Initial colony density at surface & 970 \\
\hline InitColonyDenBot, $\mathrm{kg} \mathrm{m}^{-3}$ & Initial colony density at bottom & 955 \\
\hline CompIrad, $W m^{-2}$ & Compensation irradiance for density increase & 5.45 \\
\hline CoeffDenIncr $r_{1}, s^{2} m^{-3}$ & Coefficient 1 of density increase equation & $5.33 \times 10^{-5}$ \\
\hline CoeffDenIncr $\mathrm{kg} \mathrm{m}^{-3} \mathrm{~s}^{-1}$ & Coefficient 2 of density increase equation & $-2.75 \times 10^{-4}$ \\
\hline CoeffDenDecr ${ }_{1}, s^{-1}$ & Coefficient 1 of density decrease equations & $1.58 \times 10^{-5}$ \\
\hline CoeffDenDecr, $\mathrm{kg} \mathrm{m}^{-3} \mathrm{~s}^{-1}$ & Coefficient 2 of density decrease equations & $1.64 \times 10^{-2}$ \\
\hline MinDenDecrSurf, $\mathrm{kg} \mathrm{m}^{-3}$ & $\begin{array}{l}\text { Minimum allowable density at the surface for use } \\
\text { in density decrease equation }\end{array}$ & 1080 \\
\hline MinDenDecrBot, $\mathrm{kg} \mathrm{m}^{-3}$ & $\begin{array}{l}\text { Minimum allowable density at the bottom for use } \\
\text { in density decrease equation }\end{array}$ & 980 \\
\hline DenCorrection, $\mathrm{kg} \mathrm{m}^{-3}$ & Correction factor for density decrease equation & 67 \\
\hline DepthLimOnOff, 1 or 0 & $\begin{array}{l}\text { If on, sets a maximum depth beyond which } \\
\text { phytoplankton cannot migrate }\end{array}$ & OFF \\
\hline DepthLim, $m$ & $\begin{array}{l}\text { If DepthLimOnOffis on, sets maximum migration } \\
\text { depth }\end{array}$ & - \\
\hline LossFraction & $\begin{array}{l}\text { Fraction of phytoplankton that settles out rather } \\
\text { than resuspends }\end{array}$ & 0.05 \\
\hline
\end{tabular}

Model output was compared to data collected at Dexter Reservoir during model development. Chlorophyll a profiles were collected on nine different days at two sites in the reservoir: the east basin site near the Lowell Covered Bridge and the west basin site at the downstream end of the reservoir. Continuous dissolved oxygen (D0) data was collected at two different depths at Dexter Dam during 2016. Continuous temperature profiles were collected at Dexter Dam in 2016 and 2017 and at the east basin site in 2017 (Figure 4-1). Model segment 10 was used for 
comparison with data from the east basin sampling site and model segment 21 was used for comparison with data from the west basin sampling site.

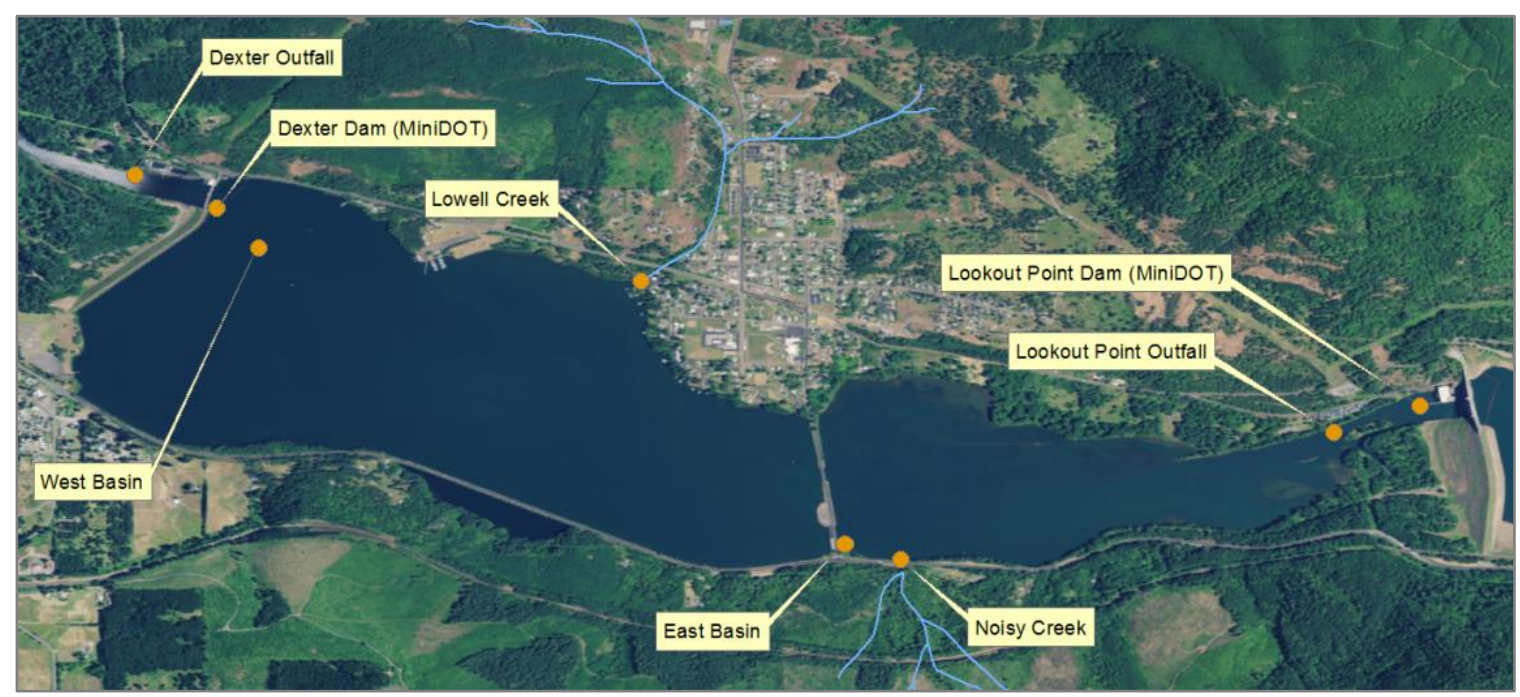

Figure 4-1 Map of Dexter Reservoir showing data collection sites, extracted from Berger et al. (2018)

\subsubsection{Results}

Model output from a control run using a constant, negative (upward) settling velocity showed some periodicity in vertical concentration profiles of migrating algae. A time series of Algal Group 2 concentration in model segment 10, corresponding to the east basin sampling site, shows high concentration at the surface throughout midday and lower, more vertically uniform concentrations during the night (Figure 4-2). This could be due in part to inflow into Dexter Reservoir from the upstream Lookout Point Reservoir. During the period shown in Figure 4-2, releases from Lookout Point Dam occurred in the afternoon and evening. One explanation for these model results is that algae concentration increases during the day while growing conditions are optimal, then starts to diffuse as velocity and 
turbulent diffusion increase due to flow from Lookout Point Dam later in the day. After these inputs stop and water becomes calmer, algae accumulate at the water surface.

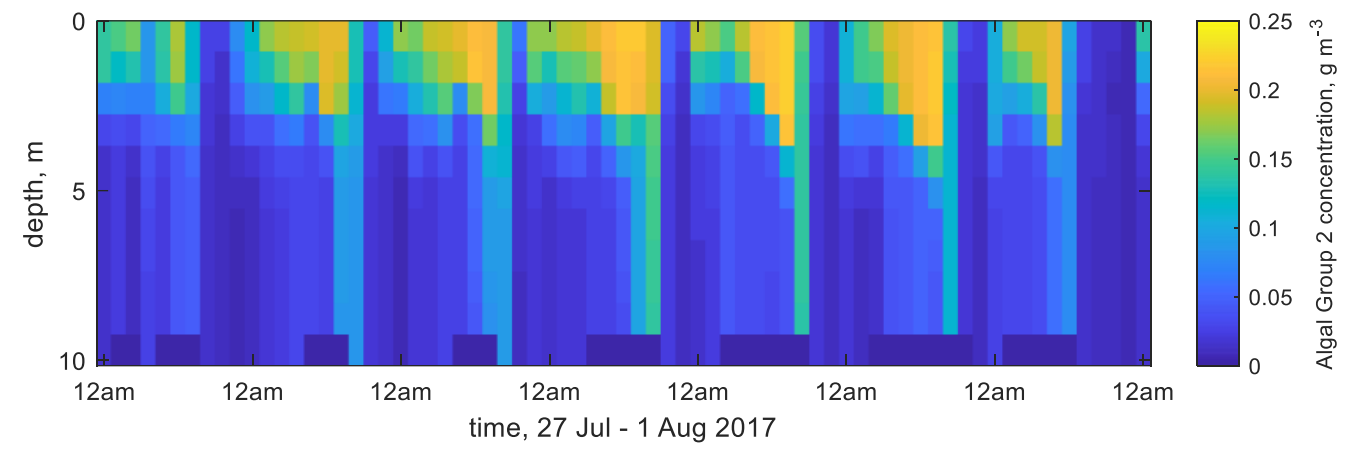

Figure 4-2 Predicted concentration of Algal Group 2 in segment 10 (east basin sampling site) using constant settling velocity

A time-series plot of Algal Group 2 concentration in model segment 21, representing the west basin sampling site, show less extreme daily changes than in segment 10 (Figure 4-3). The water here is approximately $10 \mathrm{~m}$ deeper than in segment 10 and it is farther from the upstream inflow at Lookout Point Dam. The daily variation could be due to a combination of changes throughout the day in flow from Lookout Point Dam, flow out of Dexter Dam, wind velocity, and algal growth kinetics.

Chlorophyll a concentration profiles measured at the east basin site show a tendency to have a maxima at the surface in the summer (Figure 4-4 \& Figure 4-5). In the fall, concentration is more uniform in vertical profiles (Figure 4-6). While profile data are sparse, it appears that the chlorophyll a concentration gradient is not as steep at the west basin site nor is there as pronounced a surface maxima as at 
the east basin site (Figure 4-4 \& Figure 4-5). The large root mean squared error (RMSE) in chlorophyll a concentration at segment 10 is due to a cyanobacteria bloom event in late August, 2016 that the model does not capture (Table 4-7; Berger et al., 2018). Using a constant settling velocity for Algal Group 2, the model typically over-predicts chlorophyll a concentration at the west basin site and sometimes predicts a steep gradient and surface maxima not seen in the data (Figure 4-4Figure 4-5, Figure 4-6, \& Table 4-8). However, it correctly predicts the shape of the chlorophyll a concentration profile in the summer at the east basin site (Figure 4-5).

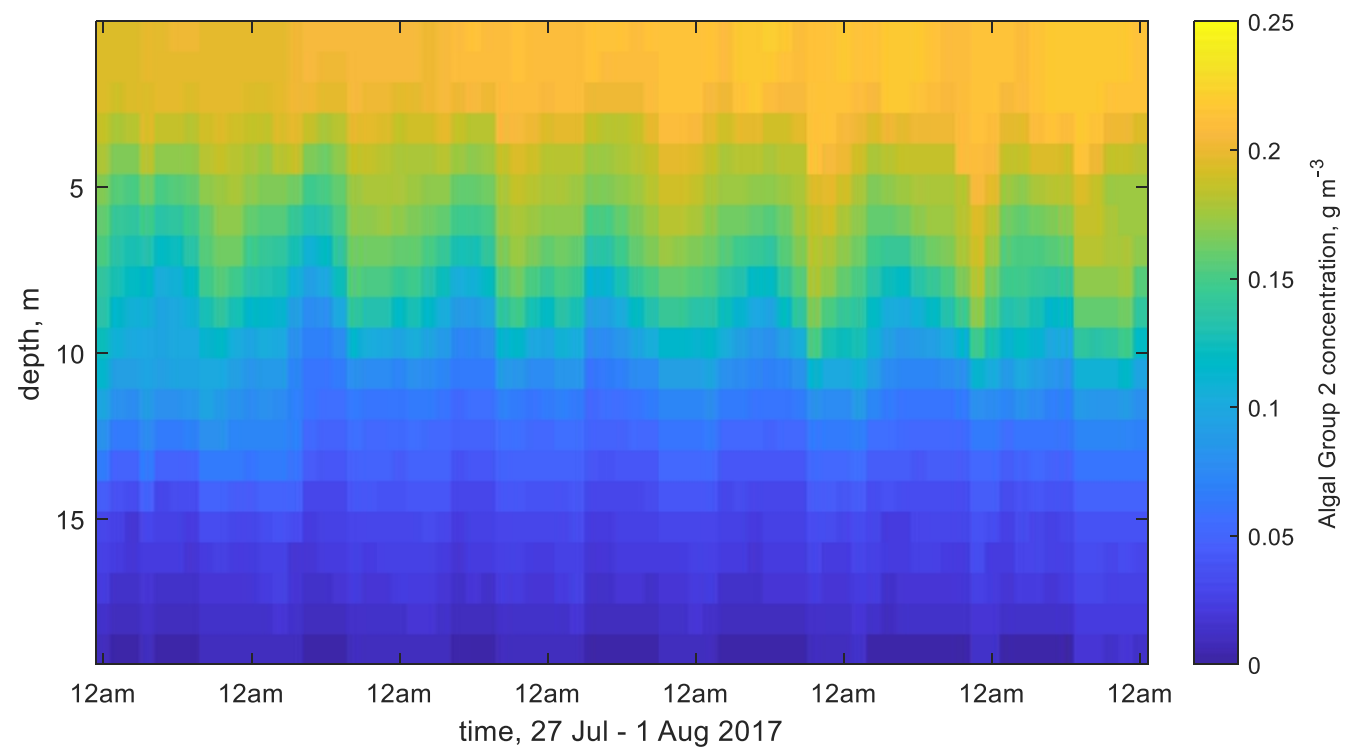

Figure 4-3 Predicted concentration of Algal Group 2 in segment 21 (west basin sampling site) using constant settling velocity 

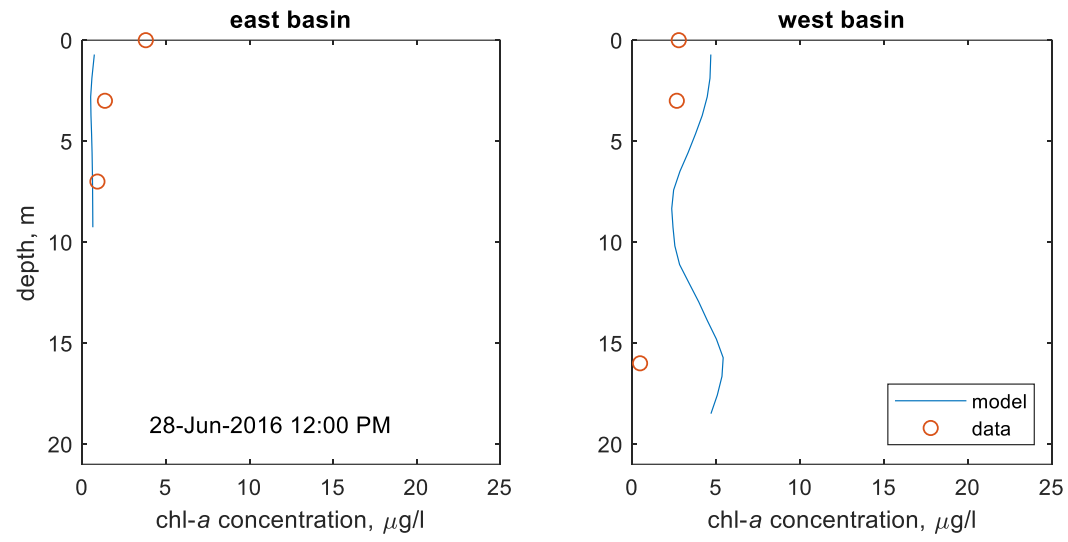

Figure 4-4 Observed and predicted chlorophyll a profiles at east basin (segment 10, left) and west basin (segment 21, right) sampling sites using constant settling velocity, 28 Jun. 2016
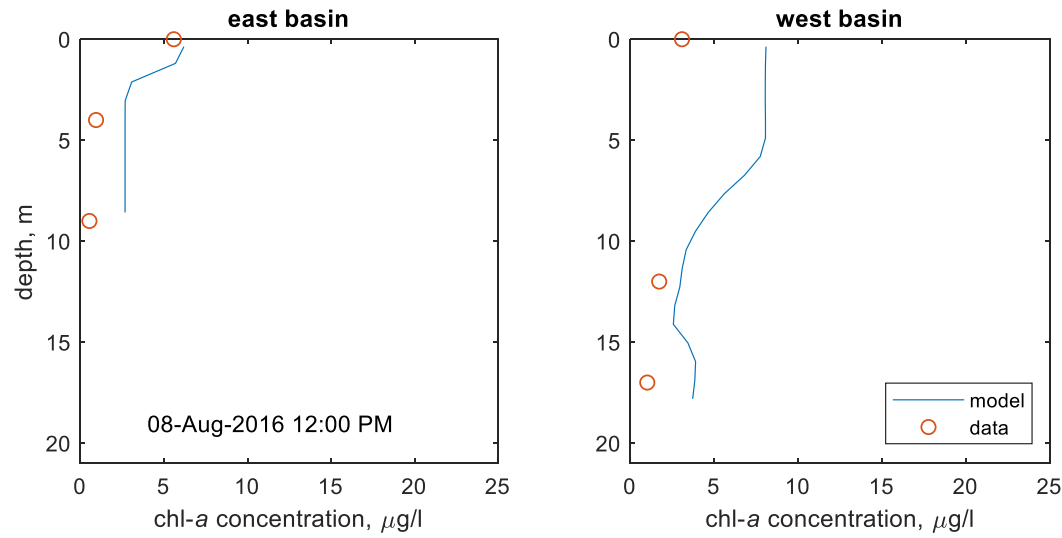

Figure 4-5 Observed and predicted chlorophyll a profiles at east basin (segment 10, left) and west basin (segment 21, right) sampling sites using constant settling velocity, 8 Aug. 2016
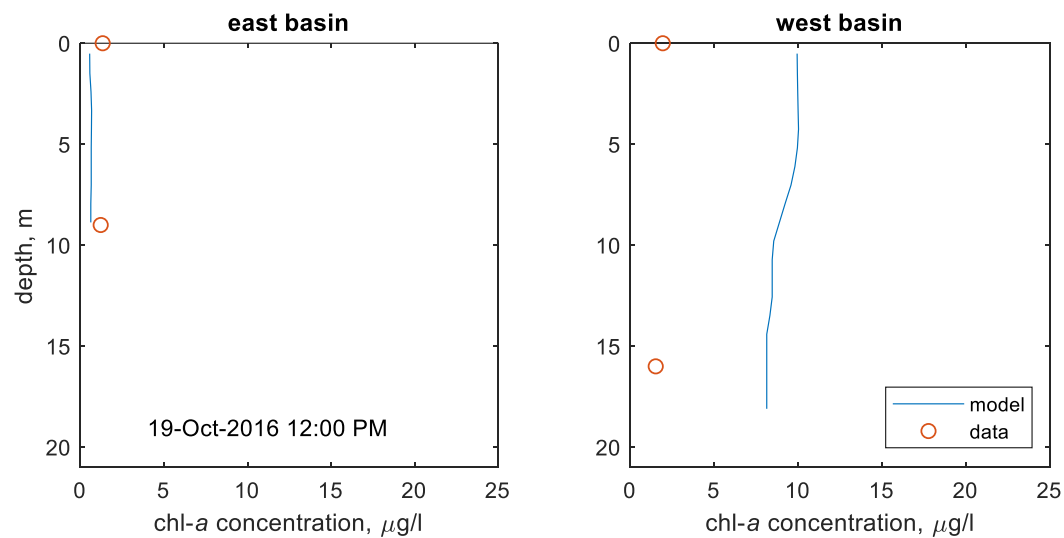

Figure 4-6 Observed and predicted chlorophyll a profiles at east basin (left) and west basin (right) sampling sites using constant settling velocity, 19 Oct. 2016 
Results from Model 1, the time-varying velocity model, predict much lower concentrations of Algal Group 2 than the control model. The same pattern in vertical distribution is predicted in segment 10 , though maximum concentrations are approximately one tenth of those in the control model (Figure 4-7). Results from segment 21 also show lower concentrations, but a diurnal vertical pattern is visible with the depth of maximum concentration varying from approximately $5 \mathrm{~m}$ deep at midnight to the surface at noon (Figure 4-8).

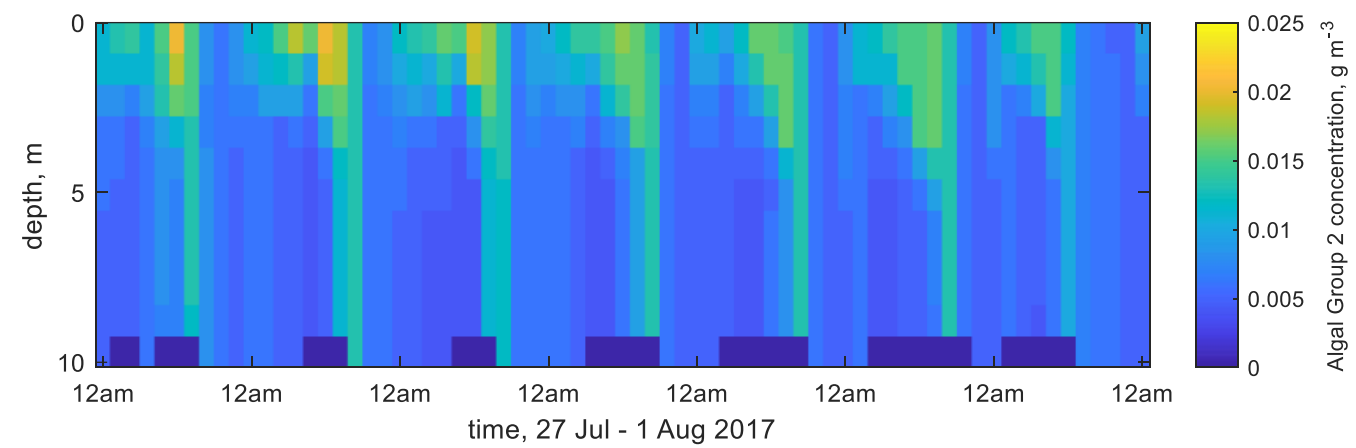

Figure 4-7 Predicted concentration of Algal Group 2 in segment 10 (east basin sampling site) using Model 1 (time-varying velocity) 


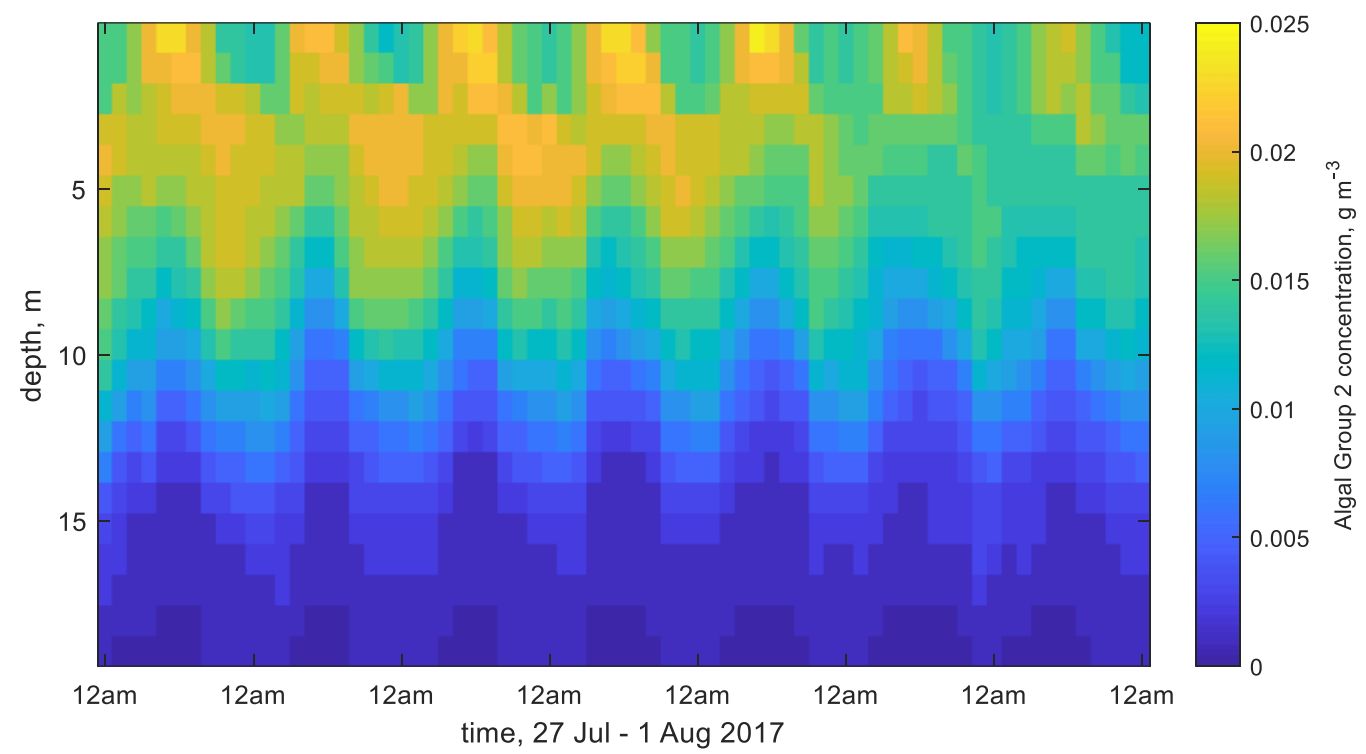

Figure 4-8 Predicted concentration of Algal Group 2 in segment 21 (west basin sampling site) using Model 1 (time-varying velocity)

Although the models including vertical migration of algae resulted in lower concentrations, error statistics were not dramatically different than the control model. AME of chlorophyll a predictions at the east basin sampling site decreased from $6.69 \mu \mathrm{g} / \mathrm{l}$ in the control model to $6.58 \mu \mathrm{g} / \mathrm{l}$ using migration Model 1 (Table 4-7). However, mean error (ME) decreased from $-6.05 \mu \mathrm{g} / \mathrm{l}$ to $-6.32 \mu \mathrm{g} / \mathrm{l}$, indicating that the model results from migration model 1 are biased lower than results from the control model. AME of chlorophyll a predictions in segment 21 and for both sites combined changed by less than $0.05 \mu \mathrm{g} / \mathrm{l}$ from the control model (Table 4-8 \& Table 4-9). Temperature and DO error statistics also changed very slightly (Table 4-10 \& Table 4-11). 
Table 4-7 Error statistics comparing output from model segment 10 to measured chlorophyll a data, east basin sampling site

\begin{tabular}{|l|r|r|r|}
\hline Chlorophyll $a$, East Basin Site & ME, $\mu \mathrm{g} / \mathrm{l}$ & AME, $\mu \mathrm{g} / \mathrm{l}$ & $\mathrm{RMSE}, \mu \mathrm{g} / \mathrm{l}$ \\
\hline Control & -6.05 & 6.69 & 23.72 \\
\hline Model 1 & -6.32 & 6.58 & 23.92 \\
\hline Model 2 & -6.34 & 6.66 & 23.95 \\
\hline Model 3 & -4.68 & 7.46 & 22.82 \\
\hline Model 4 & -6.28 & 6.66 & 23.95 \\
\hline
\end{tabular}

Table 4-8 Error statistics comparing output from model segment 21 to measured chlorophyll a, west basin sampling site

\begin{tabular}{|l|r|r|r|}
\hline Chlorophyll $\boldsymbol{a}$, West Basin Site & ME, $\mu \mathrm{g} / \mathbf{l}$ & AME, $\boldsymbol{\mu g} / \mathbf{l}$ & RMSE, $\mu \mathrm{g} / \mathbf{l}$ \\
\hline Control & 3.07 & 3.30 & 4.37 \\
\hline Model 1 & 3.08 & 3.32 & 4.34 \\
\hline Model 2 & 3.05 & 3.30 & 4.31 \\
\hline Model 3 & 3.48 & 3.69 & 5.73 \\
\hline Model 4 & 3.05 & 3.26 & 4.33 \\
\hline
\end{tabular}

Table 4-9 Error statistics comparing model output to measured chlorophyll a, both sampling sites

\begin{tabular}{|l|r|r|r|}
\hline Chlorophyll $a$, Total & ME, $\mu \mathrm{g} / \mathrm{l}$ & AME, $\mu \mathrm{g} / \mathrm{l}$ & RMSE, $\mu \mathrm{g} / \mathrm{l}$ \\
\hline Control & -1.26 & 4.91 & 16.65 \\
\hline Model 1 & -1.39 & 4.87 & 16.78 \\
\hline Model 2 & -1.41 & 4.90 & 16.80 \\
\hline Model 3 & -0.39 & 5.48 & 16.27 \\
\hline Model 4 & -1.38 & 4.88 & 16.81 \\
\hline
\end{tabular}

Table 4-10 Error statistics comparing model output to dissolved oxygen (DO) data

\begin{tabular}{|l|r|r|r|}
\hline D0, Total & ME, mg/l & AME, $\mathrm{mg} / \mathbf{l}$ & RMSE, $\mathrm{mg} / \mathbf{l}$ \\
\hline Control & 0.55 & 1.00 & 1.44 \\
\hline Model 1 & 0.53 & 0.98 & 1.41 \\
\hline Model 2 & 0.55 & 0.99 & 1.44 \\
\hline Model 3 & 0.55 & 1.00 & 1.43 \\
\hline Model 4 & 0.55 & 1.00 & 1.44 \\
\hline
\end{tabular}


Table 4-11 Error statistics comparing model output to temperature data

\begin{tabular}{|l|r|r|r|}
\hline Temperature, Total & \multicolumn{1}{|c|}{ ME, $^{\circ} \mathrm{C}$} & AME, & \\
& & \\
\hline Control & -0.30 & 0.68 & 1.03 \\
\hline Model 1 & -0.30 & 0.69 & 1.04 \\
\hline Model 2 & -0.30 & 0.69 & 1.04 \\
\hline Model 3 & -0.30 & 0.69 & 1.04 \\
\hline Model 4 & -0.30 & 0.69 & 1.04 \\
\hline
\end{tabular}

Results from model runs using migration Model 2 are similar to those from runs using Model 1, but with lower predicted concentration values at both locations (Figure 4-9 \& Figure 4-10). A higher migration amplitude was used in Model 2 (0.75 m versus $0.60 \mathrm{~m}$ ), which causes the algae to move deeper in the water column, resulting in less time spent in optimal light conditions and therefore less population growth. Error statistics were very similar to those from the control model, with the exception of chlorophyll a being more under-predicted at segment 10 (Table 4-7, Table 4-8, Table 4-9, Table 4-10, \& Table 4-11).

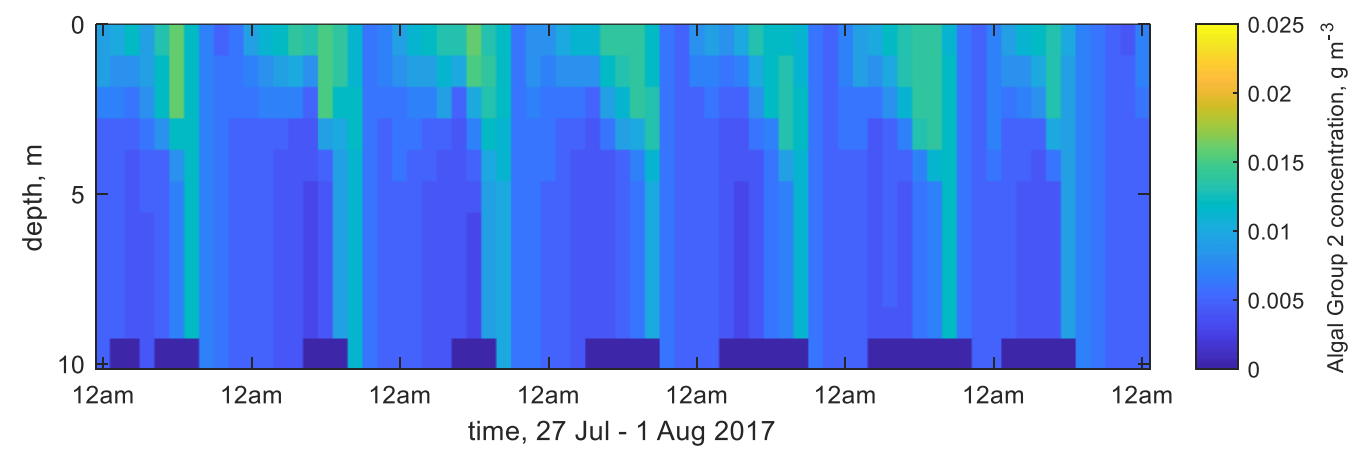

Figure 4-9 Predicted concentration of Algal Group 2 in segment 10 (east basin sampling site) using Model 2 (Belov \& Giles [1997]) 


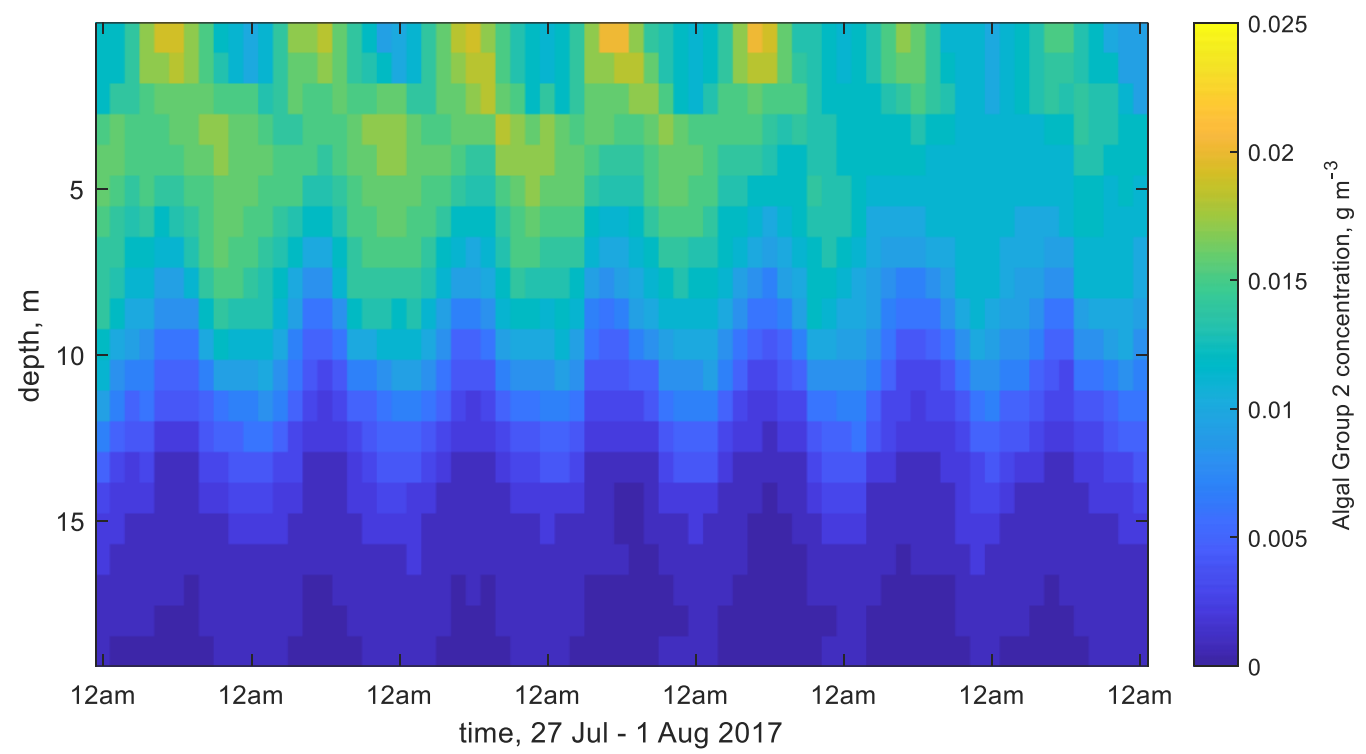

Figure 4-10 Predicted concentration of Algal Group 2 in segment 21 (west basin sampling site) using Model 2 (Belov \& Giles [1997])

The light function migration model with time decay (Model 3) predicted higher concentrations of Algal Group 2 than the predefined velocity models, but still lower than the control model. Predicted concentrations were higher in segment 21 than in segment 10 , while in the control model and time-varying velocity models they were more similar between the two locations (Figure 4-11 \& Figure 4-12). In segment 21 , algae migrate between approximately $10 \mathrm{~m}$ deep and the water surface, but the maximum concentration does not always reach the surface. Error statistics from this model show that chlorophyll a predictions are on average higher than in the control model. At the east basin site, the ME is less negative than in the control model, while at the west basin site it is more positive (Table 4-7\& Table 4-8). However, AMEs of chlorophyll a predictions are higher at both sites. Temperature and D0 error statistics are similar to the other models (Table 4-10 \& Table 4-11). 


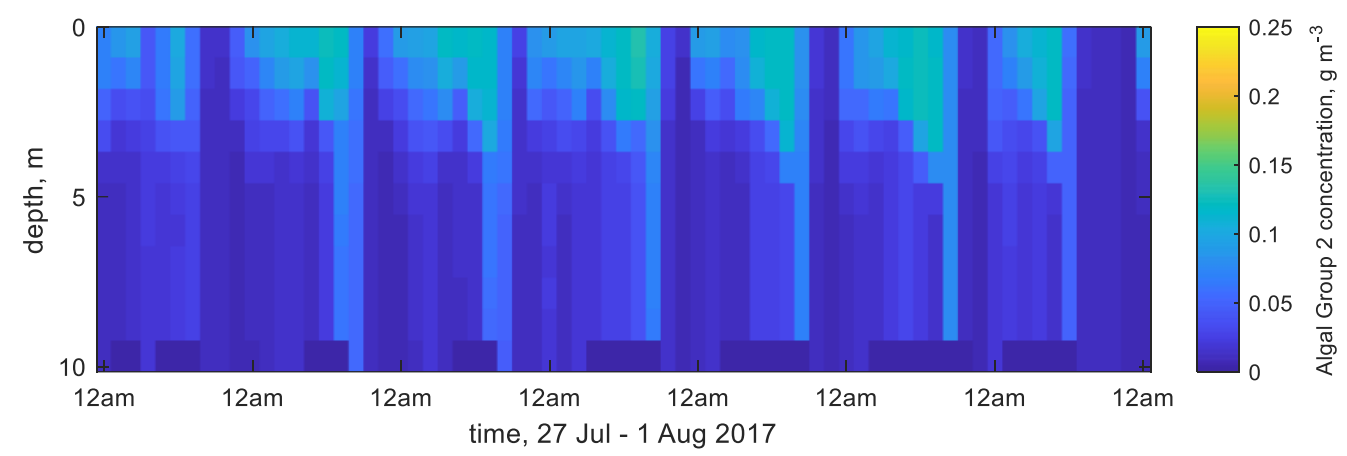

Figure 4-11 Predicted concentration of Algal Group 2 in segment 10 (east basin sampling site) using Model 3 (light function with time decay)

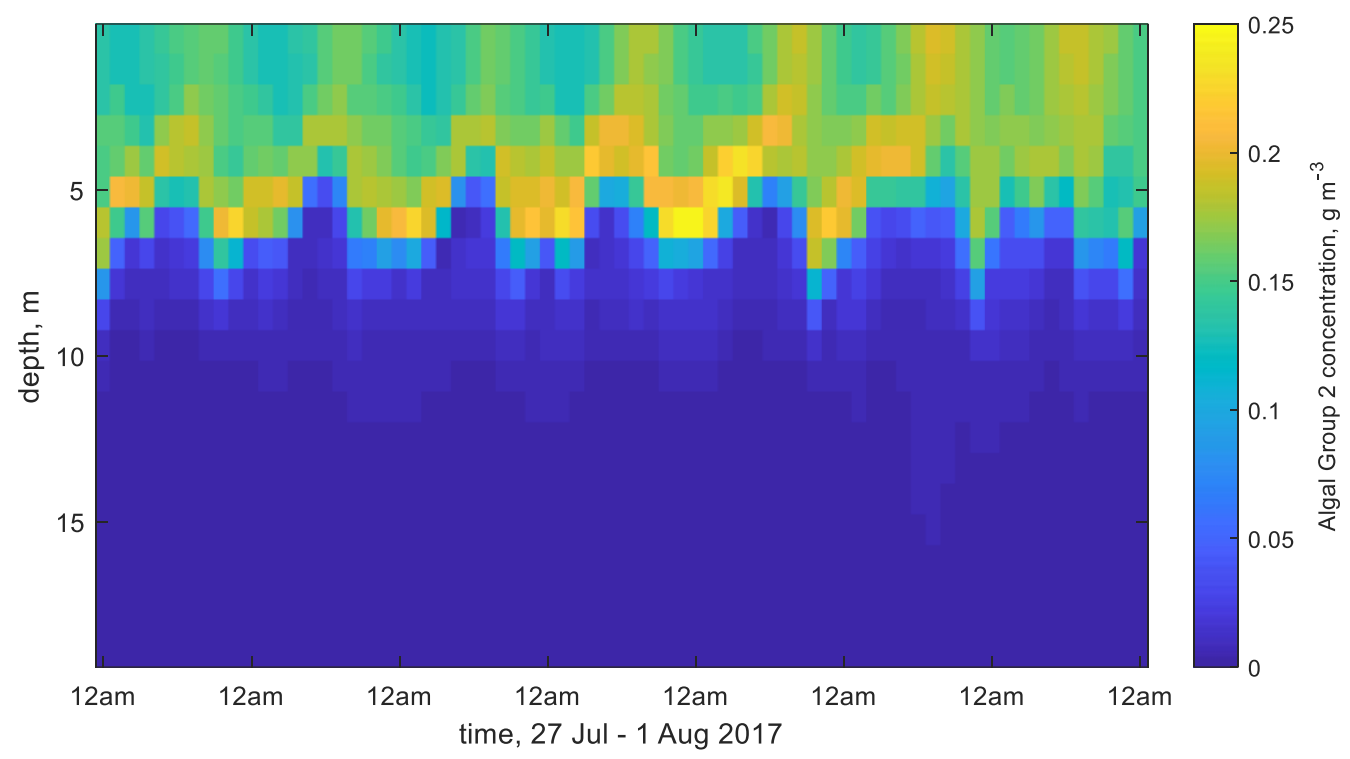

Figure 4-12 Predicted concentration of Algal Group 2 in segment 21 (west basin sampling site) using Model 3 (light function with time decay)

Results from migration Model 4 (the Visser et al. [1997] model) predict much lower concentrations in segment 10 than in segment 21 (Figure 4-13 \& Figure 4-14, note change in axis scales). Similar to predictions from the other models, there is an obvious vertical migration pattern in segment 21 (Figure 4-14). However, the highest concentrations stay mostly between $4 \mathrm{~m}$ and $11 \mathrm{~m}$ deep and concentration 
at the surface is typically very low. Predicted concentrations were greater than those from the predefined velocity models (Models $1 \& 2$ ) but lower than predictions from the control model and Model 3. Error statistics show the same pattern as in other models, with values relatively close to those from the control model and a more negative ME for chlorophyll $a$ in segment 10 (Table 4-7, Table 4-8, Table 4-9, Table 4-10, \& Table 4-11).

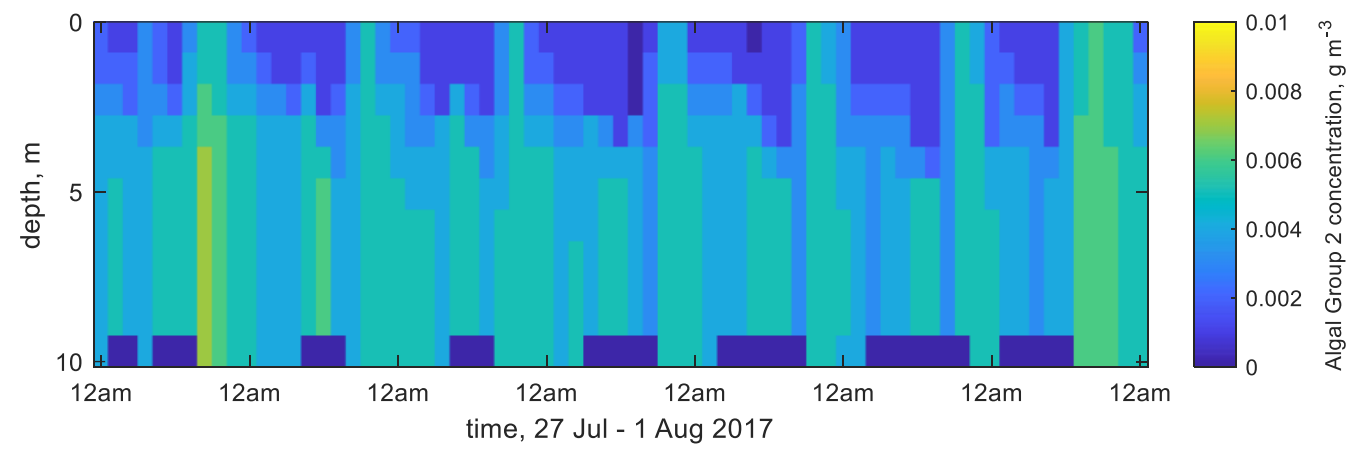

Figure 4-13 Predicted concentration of Algal Group 2 in segment 10 (east basin sampling site) using Model 4 (Visser et al. [1997]) 


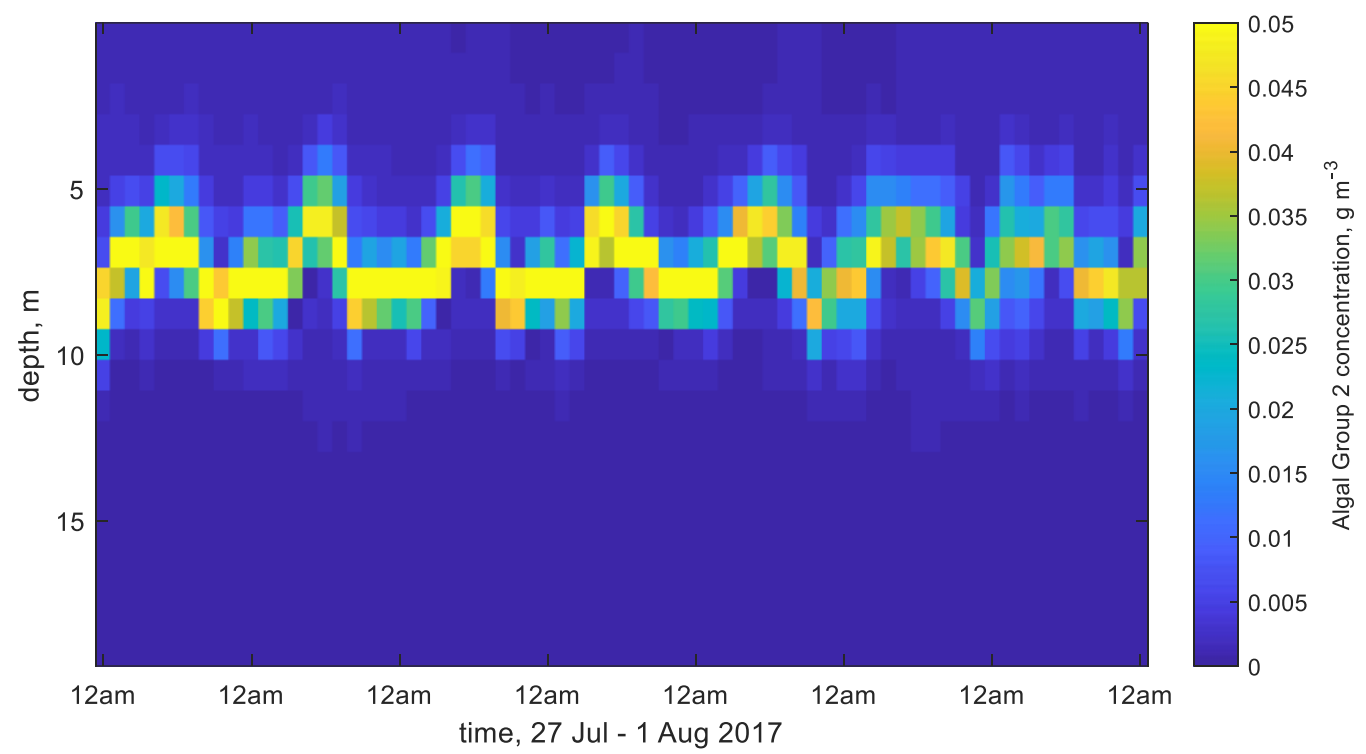

Figure 4-14 Predicted concentration of Algal Group 2 in segment 21 (west basin sampling site) using Model 4 (Visser et al. [1997])

Additional plots of model output using vertical migration Model 3 show its effect on model predictions. More variation is predicted in the chlorophyll $a$ concentration profiles in the summer at the west basin site compared to the model with constant settling velocity (Figure 4-15 \& Figure 4-16). The shape of the concentration profile at the east basin in August is better captured using Model 3 (Figure 4-16), while concentration at the west basin site is over-predicted at the surface in August and October (Figure 4-16 \& Figure 4-17). 

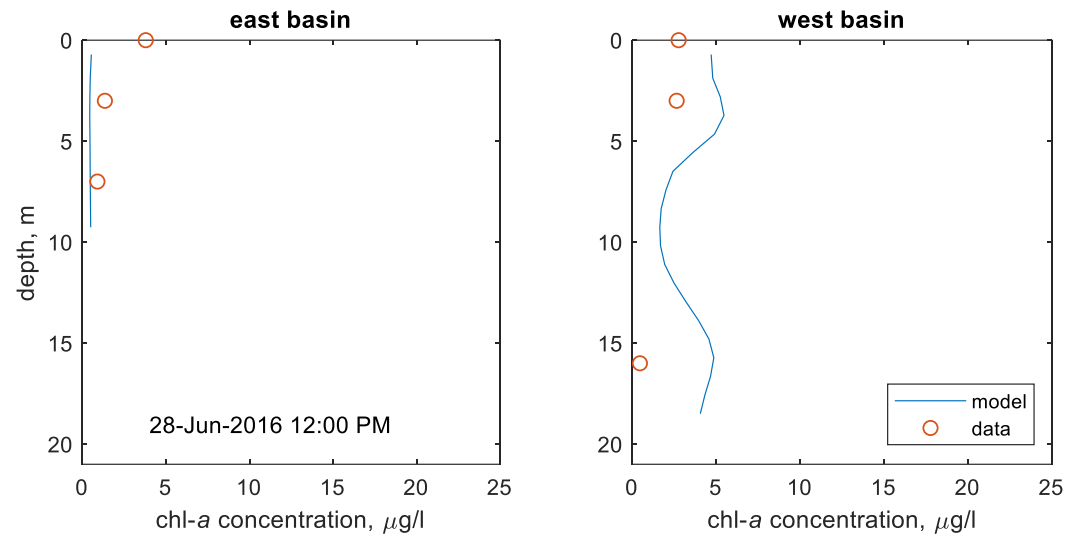

Figure 4-15 Observed and predicted chlorophyll a profiles at east basin (segment 10, left) and west basin (segment 21, right) sampling sites using Model 3 (light function with time decay), 28 Jun. 2016
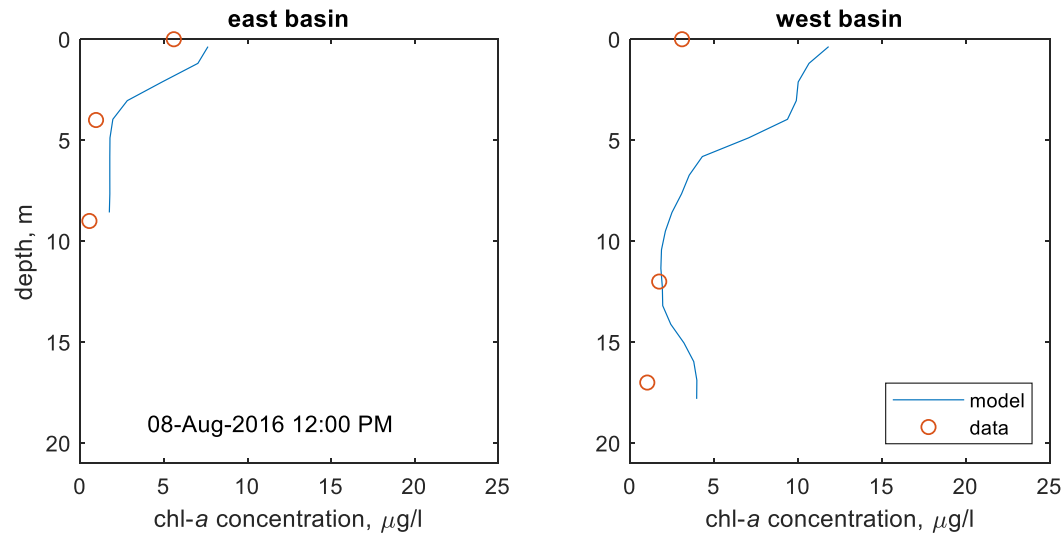

Figure 4-16 Observed and predicted chlorophyll a profiles at east basin (segment 10, left) and west basin (segment 21, right) sampling sites using Model 3 (light function with time decay), 8 Aug. 2016
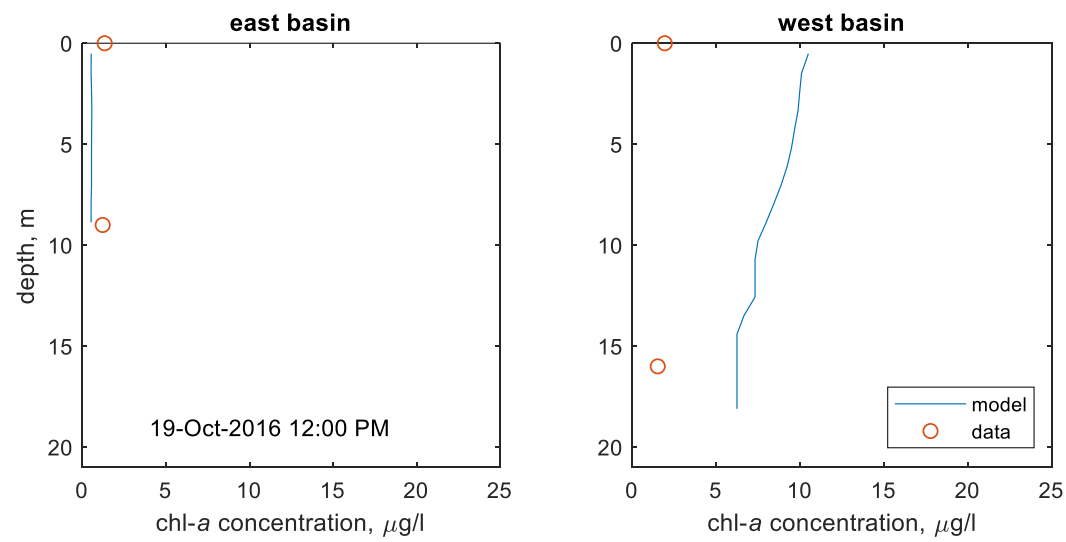

Figure 4-17 Observed and predicted chlorophyll a profiles at east basin (segment 10, left) and west basin (segment 21, right) sampling sites using Model 3 (light function with time decay), 19 Oct. 2016 
Longitudinal profiles of the reservoir show how the predicted concentration of Algal Group 2 changes over one day using vertical migration Model 3. At midnight on July 29, 2017, the highest predicted concentration of Algal Group 2 is several meters below the water surface and thermal stratification is predicted at the downstream end of the reservoir (Figure 4-18). Six hours later, predicted surface temperatures are slightly lower and predicted algae concentrations have increased at the upstream end of the reservoir (Figure 4-19). At noon, more thermal stratification and higher algae concentrations are predicted at the upstream end of the reservoir. The predicted algae concentration at the downstream end has started to move towards the surface (Figure 4-20). In the evening (6:00pm), the algae at the downstream end and near the middle of the reservoir is predicted to be more vertically diffuse (Figure 4-21). At midnight on July 30, the predicted algae concentration shows a maxima below the surface, similar to the previous night (Figure 4-22). 


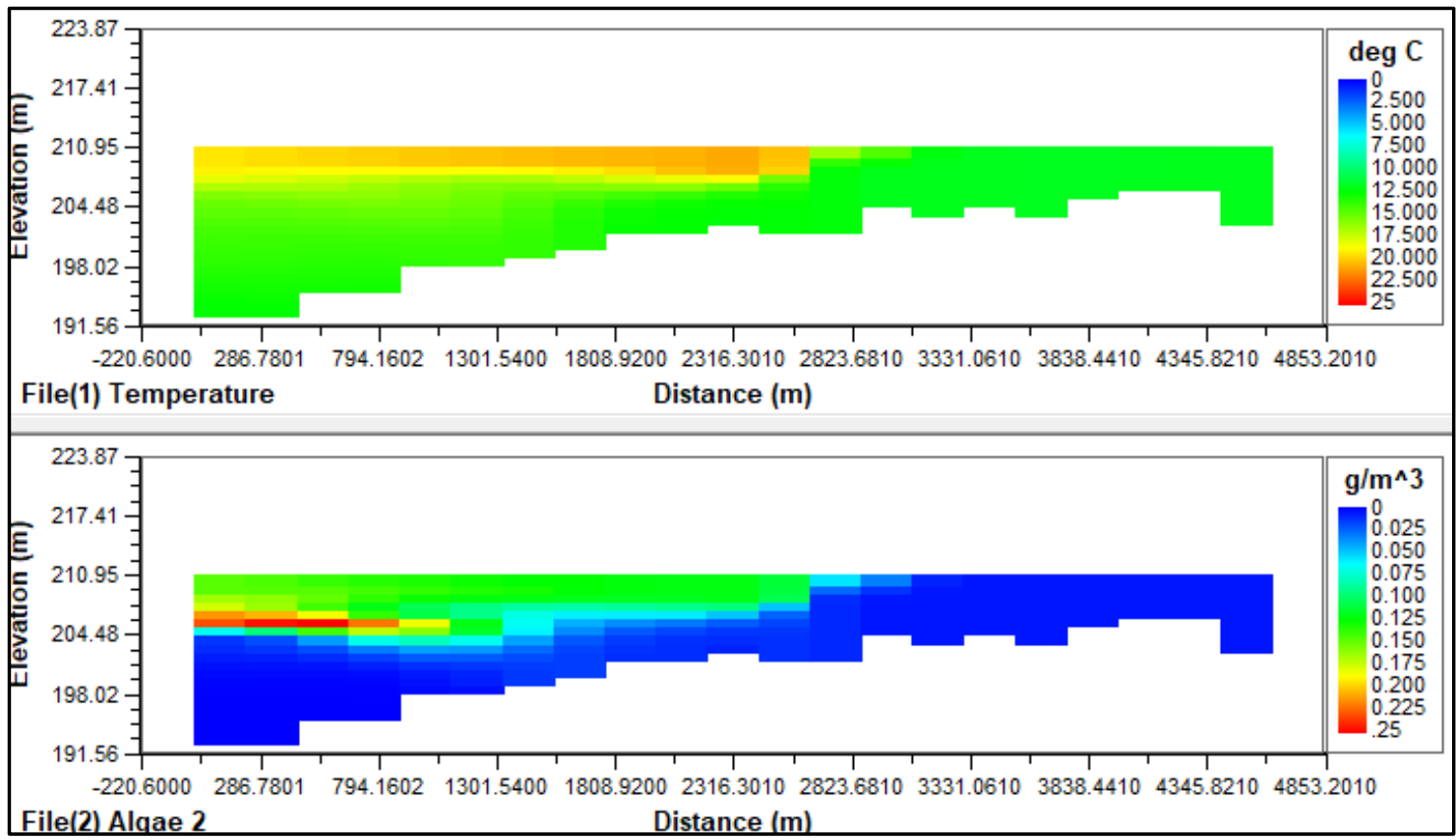

Figure 4-18 Profile view of predicted temperature (upper frame) and concentration of Algal Group 2 (lower frame) in Dexter reservoir using Model 3 (light function with time decay), 12:00am, 29 July 2017

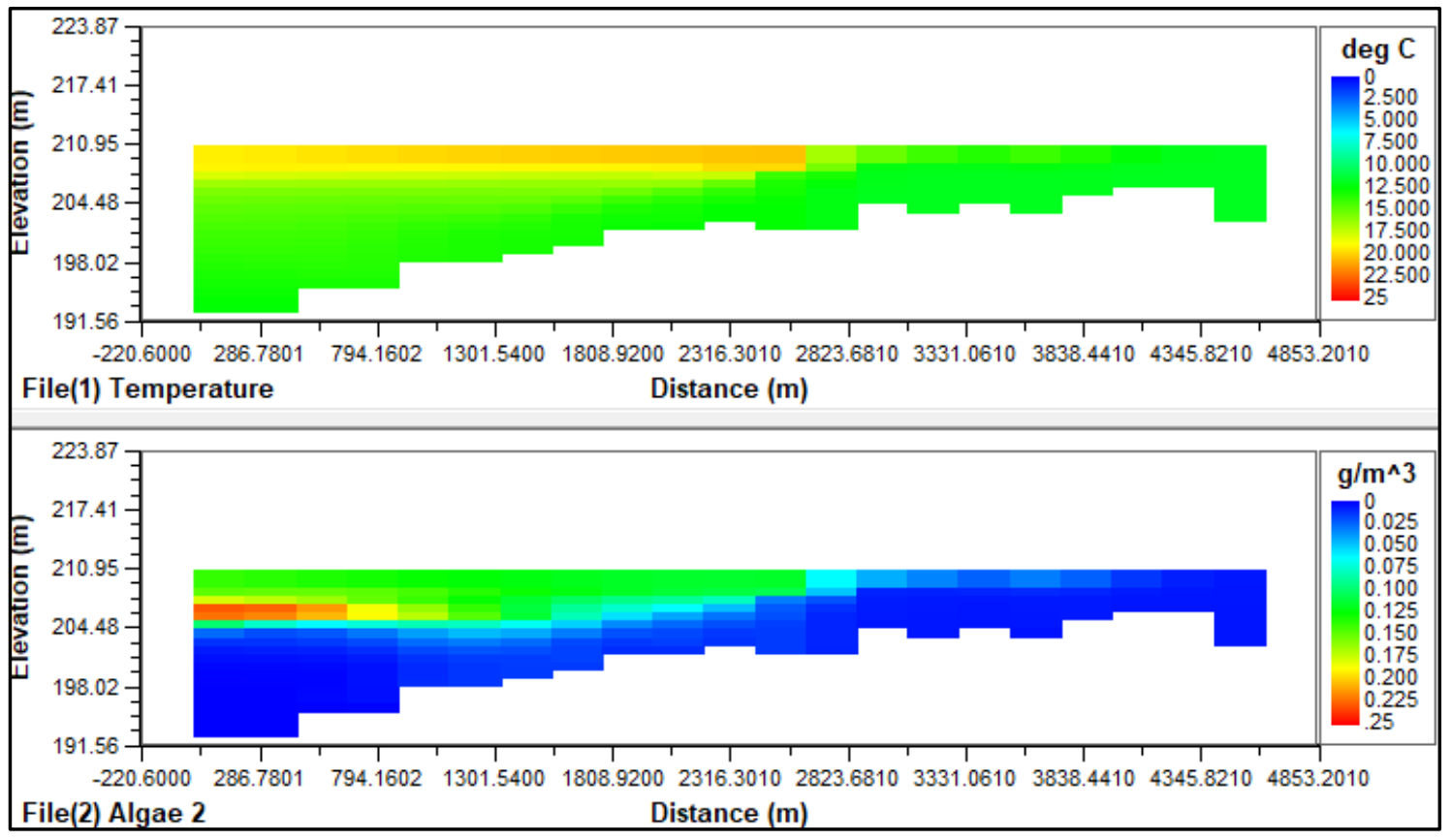

Figure 4-19 Profile view of predicted temperature (upper frame) and Algal Group 2 concentration (lower frame) in Dexter reservoir using Model 3 (light function with time decay), 6:00am, 29 July 2017 


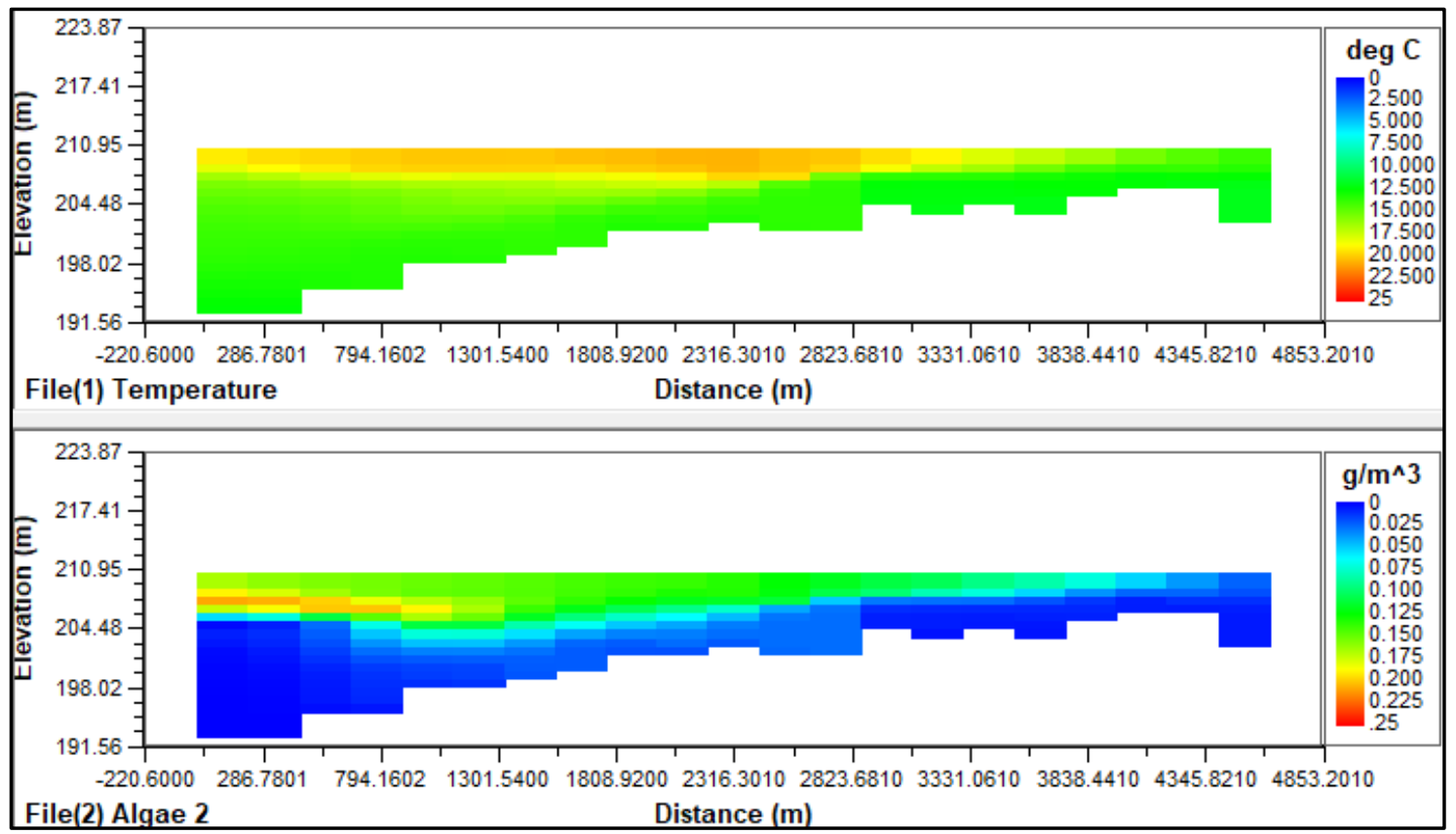

Figure 4-20 Profile view of predicted temperature (upper frame) and concentration of Algal Group 2 (lower frame) in Dexter reservoir using Model 3 (light function with time decay), 12:00pm, 29 July 2017

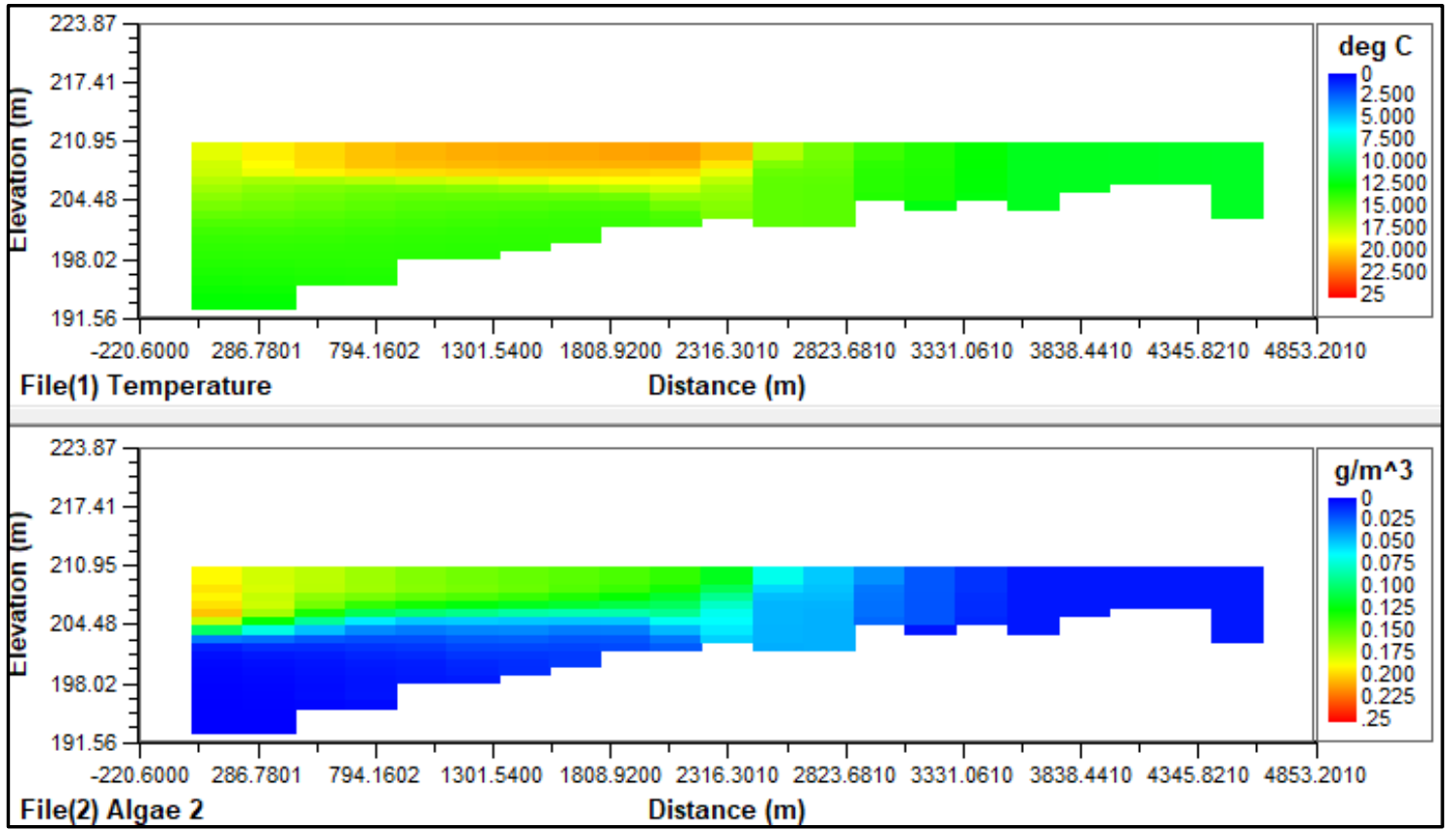

Figure 4-21 Profile view of predicted temperature (upper frame) and concentration of Algal Group 2 (lower frame) in Dexter reservoir using Model 3 (light function with time decay), 6:00pm, 29 July 2017 


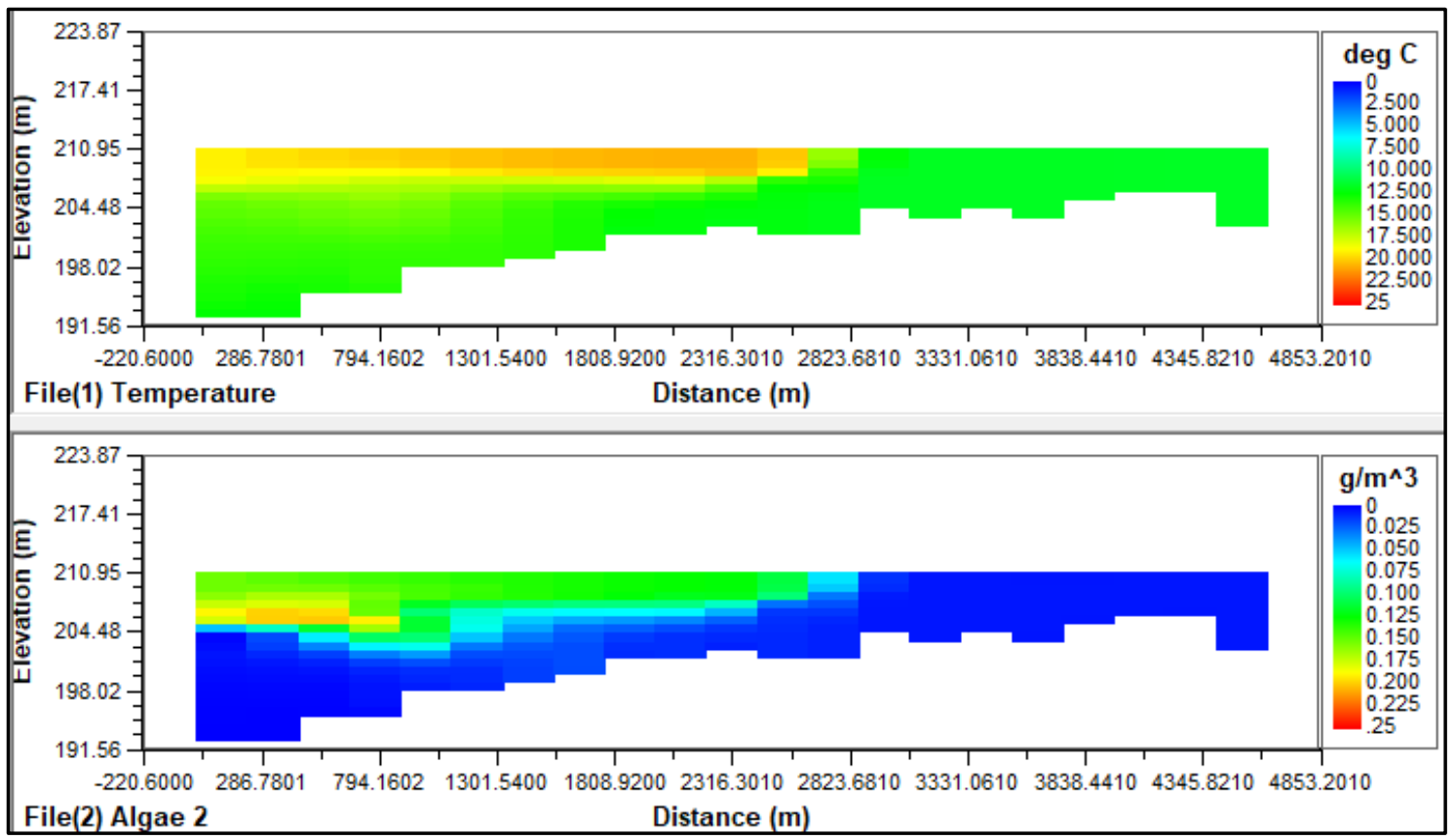

Figure 4-22 Profile view of predicted temperature (upper frame) and concentration of Algal Group 2 (lower frame) in Dexter reservoir using Model 3 (light function with time decay), 12:00am, 30 July 2017

\subsubsection{Discussion and Conclusions}

Results from testing the different cyanobacteria vertical migration models with a CE-QUAL-W2 model of Dexter Reservoir show that all are capable of predicting diurnal vertical migration of algae. However, some of the daily patterns predicted could be due to other factors present in the existing model such as hydrodynamics and growth kinetics. The migration models predict lower chlorophyll a concentrations than the existing model, but for the most part error statistics did not change. This could be addressed by additional calibration, such as adjusting the saturating light intensity which is used to predict migration velocities in Models 3 and 4. No other parts of the Dexter Reservoir model were adjusted for 
these tests, and it is possible that recalibrating the model with the addition of the migration models would lead to better results.

The comparison of model output to chlorophyll a data also presents a challenge. Vertical migration models were applied to one of the three algal groups included in the model, but it is possible that more or less of the phytoplankton in the reservoir perform vertical migration than was modeled here. Some error in chlorophyll a predictions could be due to this. Additionally, sparse chlorophyll a profiles limit the amount of calibration that can be done on vertical migration models. Profiles were collected at three-week intervals at two or three depths, but sub-daily sampling is necessary to capture diurnal vertical migration patterns.

A well-calibrated model of Dexter Reservoir that includes cyanobacteria vertical migration could be used to test different management scenarios to prevent or control HABs. For example, management strategies that increase vertical mixing and disturb the stable epilimnion where cyanobacteria accumulate could be modeled in CE-QUAL-W2. One way to increase vertical mixing would be to change the timing of releases from Lookout Point Dam. During the summer period modeled here, Lookout Point Dam released water only in the afternoon and evening. This could allow water to stratify and cyanobacteria to float to the top between releases. More frequent releases would reduce the amount of time the water is calm and stratified. However, Lookout Point Dam is used for power generation, which requires releasing water at certain times. Another strategy would be to add a 
structure that would induce more vertical mixing, such as a curtain weir. Forcing water to flow around a curtain weir would increase turbulence and vertical mixing. 


\section{Chapter 5: Conclusions}

The models of cyanobacteria vertical migration developed here were based on either sinusoidal, diurnal vertical movement or density change as a result of photosynthesis. The predefined velocity models based on sinusoidal motion were simple to implement and often gave good results, especially at lower values of vertical diffusion. The models of density change showed more daily variation but involved many calibration parameters. Often these models made realistic predictions, but required extra adjustments. The density-change models represent a complex biological system reduced to several equations, so simplifications and assumptions have to be made. These models capture the natural processes that are happening, but erroneous predictions can result from improper calibration. Errors could also be due to neglecting an important process when simplifying a complicated system.

In tests on field data, models using both continuum and particle tracking frameworks made accurate predictions. More diffusion was seen in model results using a continuum framework; however, this could be due the numerical scheme used in preliminary models (upwind). In CE-QUAL-W2, use of the ULTIMATEQUICKEST scheme avoids some of this numerical diffusion. Use of a particle-tracking framework improved results from dynamic velocity models due to the inherent Lagrangian nature of these models. However, results were not clearly improved by using the particle-tracking framework for predefined velocity models, and the 
added complexity of such a framework may not be worthwhile for these types of models.

Assumptions and simplifications made in the preliminary models using the particle-tracking framework could prove to be problematic when applied to a real system. Particles representing cyanobacteria colonies were assumed to be spherical and to have a constant volume, and particle-particle interactions were not considered. In reality, some cyanobacteria species form colonies or filaments that grow over time and do not remain spherical. Velocity of these colonies can deviate from the velocity predicted by Stokes' law for a sphere due to irregular shapes (Reynolds, 2006). Cyanobacteria that has formed a surface scum would also not fit the assumptions of a spherical particle if colonies are stuck together in a mat formation. Incorporation of methods developed for modeling flocculation in wastewater treatment systems could address some of these issues.

One potential shortcoming of the models presented here is that they are only dependent on light intensity as a predictor of density change or vertical migration. Other factors can influence vertical migration in cyanobacteria, such as nutrients (Brookes and Ganf, 2001) and turbulence (Zhao et al., 2017). Similarly, zooplankton migration is affected by food concentration, predator abundance, temperature, and dissolved oxygen, in addition to light intensity (Larsson and Lampert, 2012).

The models developed here show that aspects of cyanobacteria and zooplankton diurnal vertical migration can be simulated using simple input 
variables such as solar irradiance. The application of the cyanobacteria vertical migration models to a model of Dexter Reservoir suggests that they are able to predict vertical migration in CE-QUAL-W2. Error statistics did not immediately improve with use of vertical migration models, but this could indicate a need to recalibrate the Dexter Reservoir model for use with the new migration models.

\subsection{Future Work}

More work could be done to improve the models of cyanobacteria vertical migration developed here. Other models could be explored, such as those that predict velocity rather than density change based on environmental inputs. This would be similar to the model of zooplankton migration by Dodson (1990) and would be a balance between the simple, time-varying velocity models and the complicated, density-change models.

The models developed here could be further calibrated and tested on other field data. For example, the two studies used to test the models in this work were both conducted in shallow waterbodies within the Three Gorges Reservoir area dominated by Microcystis species. It would be helpful to test the models on a different system, such as a deeper lake or reservoir, in another part of the world and perhaps on a different type of migrating cyanobacteria. This would give a better understanding of how to calibrate the models for various uses. The issue of modeling all chlorophyll a concentration as being due to cyanobacteria could be addressed by a correction factor that accounts for the chlorophyll a contributed by 
other species. This would require comparison of species analysis to chlorophyll $a$ concentration, such as was reported by Wang et al. (2011) and Cui et al. (2016).

The models implemented into CE-QUAL-W2 would benefit from further calibration and test cases. This could include applying them to other waterbodies or using additional verification data to test whether the models are making accurate predictions. Chlorophyll a profile data or remotely sensed images showing time of algal blooms are two possible sources of data.

The preliminary zooplankton models presented here should be tested on field data as was done with cyanobacteria models in the study. This would give information on how the models perform and which models would be useful to use in CE-QUAL-W2. Once suitable models are developed, they could be implemented into CE-QUAL-W2 as were the phytoplankton migration models.

Finally, the models of cyanobacteria and zooplankton developed here using the particle-tracking framework should be incorporated into the existing particletracking algorithm of CE-QUAL-W2. This would be beneficial to modeling cyanobacteria because colony density changes are more realistically represented by following a particle through time and space as opposed to modeling the predicted density at a particular point in space.

Making advances toward more accurately modeling cyanobacteria and zooplankton movement and behavior will allow for better models of lakes and 
reservoirs and better predictions of HABs. Better predictions could help with prevention and management of blooms, making waterbodies safer and cleaner. 


\section{References}

Andersen, V., and Nival, P. (1991). "A model of the diel vertical migration of zooplankton based on euphausiids.” Journal of Marine Research, 49(1), 153175.

Aparicio Medrano, E., Uittenbogaard, R. E., Dionisio Pires, L. M., van de Wiel, B. J. H., and Clercx, H. J. H. (2013). "Coupling hydrodynamics and buoyancy regulation in Microcystis aeruginosa for its vertical distribution in lakes." Ecological Modelling, 248, 41-56.

Belov, A. P., and Giles, J. D. (1997). “Dynamical model of buoyant cyanobacteria.” Hydrobiologia, (349), 11.

Berger, C., Wells, S., Miller, R., Sytsma, M., and Strecker, A. (2018). Dexter Reservoir Model Development and Calibration Report. Prepared for ERDC Contracting Office U.S. Army Corps of Engineers Engineer Research and Development Center, Water Quality Research Group and Center for Lakes and Reservoirs, Department of Civil and Environmental Engineering and Environmental Science and Management, Portland State University.

Bonnet, M. P., and Poulin, M. (2002). "Numerical modelling of the planktonic succession in a nutrient-rich reservoir: Environmental and physiological factors leading to Microcystis aeruginosa dominance." Ecological Modelling, 156(2-3), 93-112.

Bormans, M., Sherman, B. S., and Webster, I. T. (1999). "Is buoyancy regulation in cyanobacteria an adaptation to exploit separation of light and nutrients?" Marine and Freshwater Research, 50(8), 897.

Boscarino, B. T., Rudstam, L. G., Loew, E. R., and Mills, E. L. (2009). "Predicting the vertical distribution of the opossum shrimp, Mysis relicta, in Lake Ontario: a test of laboratory-based light preferences." Canadian Journal of Fisheries and Aquatic Sciences, 66(1), 101-113.

Brookes, J. D., and Ganf, G. G. (2001). "Variations in the buoyancy response of Microcystis aeruginosa to nitrogen, phosphorus and light." Journal of Plankton Research, 23(12), 1399-1411.

Cao, H. S., Kong, F. X., Luo, L. C., Shi, X. L., Yang, Z., Zhang, X. F., and Tao, Y. (2006). "Effects of wind and wind-induced waves on vertical phytoplankton distribution and surface blooms of Microcystis aeruginosa in Lake Taihu." Journal of Freshwater Ecology, 21(2), 231-238. 
Chen, Y., Qian, X., and Zhang, Y. (2009). "Modelling turbulent dispersion of buoyancy regulating cyanobacteria in wind-driven currents." 2009 3rd International Conference on Bioinformatics and Biomedical Engineering, IEEE, Beijing, China, $1-4$.

Chien, Y. C., Wu, S. C., Chen, W. C., and Chou, C. C. (2013). "Model simulation of diurnal vertical migration patterns of different-sized colonies of Microcystis employing a particle trajectory approach." Environmental Engineering Science, 30(4), 179-186.

Chung, S. W., Imberger, J., Hipsey, M. R., and Lee, H. S. (2014). "The influence of physical and physiological processes on the spatial heterogeneity of a Microcystis bloom in a stratified reservoir." Ecological Modelling, 289, 133149.

Cohen, J., and Forward, R. B. J. (2009). "Zooplankton diel vertical migration - A review of proximate control." Oceanography and Marine Biology: An Annual Review, CRC Press, 89-122.

Cole, T. M., and Wells, S. A. (2018). "CE-QUAL-W2: A Two-Dimensional, Laterally Averaged, Hydrodynamic and Water Quality Model, Version 4.1." Department of Civil and Environmental Engineering, Portland State University, Portland, OR.

Cui, Y. J., Liu, D. F., Zhang, J., Yang, Z. J., Khu, S. T., Ji, D. B., Song, L. X., and Long, L. H. (2016). "Diel migration of Microcystis during an algal bloom event in the Three Gorges Reservoir, China." Environmental Earth Sciences, 75(7).

De Robertis, A. (2002). "Size-dependent visual predation risk and the timing of vertical migration: An optimization model." Limnology and Oceanography, 47(4), 925-933.

Dewar, W. K., Bingham, R. J., Iverson, R. L., Nowacek, D. P., St. Laurent, L. C., and Wiebe, P. H. (2006). "Does the marine biosphere mix the ocean?" Journal of Marine Research, 64(4), 541-561.

Dodson, S. (1990). "Predicting diel vertical migration of zooplankton." Limnology and Oceanography, 35(5), 1195-1200.

Easthope, M. P., and Howard, A. (1999). "Simulating cyanobacterial growth in a lowland reservoir." Science of the Total Environment, 241(1-3), 17-25. 
Falconer, I. R. (2005). Cyanobacterial Toxins of Drinking Water Supplies: Cylindrospermopsins and Microcystins. CRC Press.

Fiksen, Ø. (1997). "Allocation patterns and diel vertical migration: Modeling the optimal Daphnia." Ecology, 78(5), 1446-1456.

Fiksen, Ø., and Carlotti, F. (1998). "A model of optimal life history and diel vertical migration in Calanus finmarchicus." Sarsia, 83(2), 129-147.

Fristachi, A., Sinclair, J. L., Hall, S., Berkman, J. A. H., Boyer, G., Burkholder, J., Burns, J., Carmichael, W., DuFour, A., Frazier, W., Morton, S. L., O'Brien, E., and Walker, S. (2008). "Occurrence of Cyanobacterial Harmful Algal Blooms Workgroup Report." Cyanobacterial Harmful Algal Blooms: State of the Science and Research Needs, Advances in Experimental Medicine and Biology, H. K. Hudnell, ed., Springer, New York, NY, 59.

Fujiki, H., Suganuma, M., Suguri, H., Yoshizawa, S., Takagi, K., Nakayasu, M., Ojika, M., Yamada, K., Yasumoto, T., Moore, R. E., and Sugimura, T. (1990). "New Tumor Promoters from Marine Natural Products." Marine Toxins, ACS Symposium Series, American Chemical Society, 232-240.

Ganf, G. G., and Oliver, R. L. (1982). “Vertical separation of light and available nutrients as a factor causing replacement of green algae by bluegreen algae in the plankton of a stratified lake.pdf."

Guven, B., and Howard, A. (2006). "Modelling the growth and movement of cyanobacteria in river systems." Science of the Total Environment, 368(2-3), 898-908.

Han, B. P., and Straškraba, M. (1998). "Modeling patterns of zooplankton diel vertical migration." Journal of Plankton Research, 20(8), 1463-1487.

HARNESS. (2005). Harmful Algal Research and Response: A National Environmental Science Strategy 2005-2015. J. S. Ramsdell, D. M. Anderson, and P. M. Gilbert, eds., Ecological Society of America, Washington, D.C., 96.

Hedger, R. D., Olsen, N. R. B., George, D. G., Malthus, T. J., and Atkinson, P. M. (2004). "Modelling spatial distributions of Ceratium hirundnella and Microcystis spp. in a small productive British lake." Hydrobiologia, 528, 11.

Hipsey, M. R., Antenucci, J. P., Romero, J. R., and Hamilton, D. (2007). “Computational Aquatic Ecosystem Dynamics Model: CAEDYM v3." Centre for Water Research, University of Western Australia. 
Howard, A. (1994). "Problem Cyanobacterial Blooms: Explanation and Simulation Modelling." Transactions of the Institute of British Geographers, 19(2), 213.

Howard, A., Irish, A. E., and Reynolds, C. S. (1996). "A new simulation of cyanobacterial underwater movement (SCUM'96)." Journal of Plankton Research, 18(8), 1375-1385.

Hudnell, H. K. (2010). "The state of U.S. freshwater harmful algal blooms assessments, policy and legislation." Toxicon, 55(5), 1024-1034.

Ibelings, B. W., Mur, L. R., and Walsby, A. E. (1991). "Diurnal changes in buoyancy and vertical distribution in populations of Microcystis in two shallow lakes." Journal of Plankton Research, 13(2), 419-436.

Jacovides, C. P., Timvios, F. S., Papaioannou, G., Asimakopoulos, D. N., and Theofilou, C. M. (2004). "Ratio of PAR to broadband solar radiation measured in Cyprus." Agricultural and Forest Meteorology, 121(3-4), 135-140.

Jensen, O. P., Hrabik, T. R., Martell, S. J. D., Walters, C. J., and Kitchell, J. F. (2006). "Diel vertical migration in the Lake Superior pelagic community. II. Modeling trade-offs at an intermediate trophic level." Canadian Journal of Fisheries and Aquatic Sciences, 63(10), 2296-2307.

Katija, K., and Dabiri, J. O. (2009). "A viscosity-enhanced mechanism for biogenic ocean mixing." Nature, 460(7255), 624-626.

Kessler, K., and Lampert, W. (2004). "Fitness optimization of Daphnia in a trade-off between food and temperature." Oecologia, 140(3), 381-387.

Kromkamp, J. C., and Mur, L. R. (1984). "Buoyant density changes in the cyanobacterium Microcystis aeruginosa due to changes in the cellular carbohydrate content." FEMS Microbiology Letters, 25(1), 105-109.

Kromkamp, J., Konopka, A., and Mur, L. R. (1988). "Buoyancy regulation in lightlimited continuous cultures of Microcystis aeruginosa." Journal of Plankton Research, 10(2), 171-183.

Kromkamp, J., and Walsby, A. E. (1990). “A computer model of buoyancy and vertical migration in cyanobacteria." Journal of Plankton Research, 12(1), 161183. 
Kunze, E., Dower, J. F., Beveridge, I., Dewey, R., and Bartlett, K. P. (2006). "Observations of biologically generated turbulence in a coastal inlet." Science, 313.

Larsson, P., and Lampert, W. (2012). "Finding the optimal vertical distribution: Behavioural responses of Daphnia pulicaria to gradients of environmental factors and the presence of fish." Freshwater Biology, 57(12), 2514-2525.

Long, B. M., Jones, G. J., and Orr, P. T. (2001). "Cellular microcystin content in Nlimited Microcystis aeruginosa can be predicted from growth rate." Applied and Environmental Microbiology, 67(1), 278-283.

Lopez, C. B., Jewett, E. B., Dortch, Q., Walton, B. T., and Hudnell, H. K. (2008). Scientific Assessment of Freshwater Harmful Algal Blooms. Interagency Working Group on Harmful Algal Blooms, Hypoxia, and Human Health of the Joint Subcommittee on Ocean Science and Technology, Washington, D.C.

Mascarenhas, F. C. B., and Trento, A. E. (2006). "Particle-tracking method applied to transport problems in water bodies." WIT Press, 503-513.

Mieleitner, J., and Reichert, P. (2006). "Analysis of the transferability of a biogeochemical lake model to lakes of different trophic state." Ecological Modelling, 194(1-3), 49-61.

Mooij, W. M., Trolle, D., Jeppesen, E., Arhonditsis, G., Belolipetsky, P. V., Chitamwebwa, D. B. R., Degermendzhy, A. G., DeAngelis, D. L., De Senerpont Domis, L. N., Downing, A. S., Elliott, J. A., Fragoso, C. R., Gaedke, U., Genova, S. N., Gulati, R. D., Håkanson, L., Hamilton, D. P., Hipsey, M. R., 't Hoen, J., Hülsmann, S., Los, F. H., Makler-Pick, V., Petzoldt, T., Prokopkin, I. G., Rinke, K., Schep, S. A., Tominaga, K., Van Dam, A. A., Van Nes, E. H., Wells, S. A., and Janse, J. H. (2010). "Challenges and opportunities for integrating lake ecosystem modelling approaches." Aquatic Ecology, 44(3), 633-667.

Nakamura, T., Adachi, Y., and Suzuki, M. (1993). "Flotation and sedimentation of a single Microcystis floc collected from surface bloom." Water Research, 27(6), 979-983.

Ndong, M., Bird, D., Nguyen Quang, T., Kahawita, R., Hamilton, D., de Boutray, M. L., Prévost, M., and Dorner, S. (2017). "A novel Eulerian approach for modelling cyanobacteria movement: Thin layer formation and recurrent risk to drinking water intakes." Water Research, 127, 191-203. 
Nowicki, C. J., Bunnell, D. B., Armenio, P. M., Warner, D. M., Vanderploeg, H. A., Cavaletto, J. F., Mayer, C. M., and Adams, J. V. (2017). "Biotic and abiotic factors influencing zooplankton vertical distribution in Lake Huron." Journal of Great Lakes Research, 43(6), 1044-1054.

Okada, M., and Aiba, S. (1983a). "Simulation of water-bloom in a eutrophic lake: III. Modeling the vertical migration and growth of Microcystis aeruginosa." Water Research, 17(8), 883-893.

Okada, M., and Aiba, S. (1983b). "Simulation of water-bloom in a eutrophic lake-II. Reassessment of buoyancy, gas vacuole and Turgor pressure of Microcystis aeruginosa." Water Research, 17(8), 877-882.

Olsen, N. R. B., Hedger, R. D., and George, D. G. (2000). “3D Numerical modeling of Microcystis distribution in a water reservoir." Journal of Environmental Engineering, 126(10), 949-953.

Paerl, H. W., Fulton, R. S., Moisander, P. H., and Dyble, J. (2001). "Harmful freshwater algal blooms, with an emphasis on cyanobacteria." The Scientific World Journal, 1, 76-113.

Rabouille, S., and Salençon, M. (2005). "Functional analysis of Microcystis vertical migration: a dynamic model as a prospecting tool. II. Influence of mixing, thermal stratification and colony diameter on biomass production." Aquatic Microbial Ecology, 39, 281-292.

Rabouille, S., Salençon, M. J., and Thébault, J. M. (2005). "Functional analysis of Microcystis vertical migration: A dynamic model as a prospecting tool." Ecological Modelling, 188(2-4), 386-403.

Rabouille, S., Thébault, J. M., and Salençon, M. J. (2003). “Simulation of carbon reserve dynamics in Microcystis and its influence on vertical migration with Yoyo model." Comptes Rendus Biologies, 326(4), 349-361.

Recknagel, F., Cetin, L., and Zhang, B. (2008). "Process-based simulation library SALMO-00 for lake ecosystems. Part 1: Object-oriented implementation and validation." Ecological Informatics, 3(2), 170-180.

Reynolds, C. S. (1984). The ecology of freshwater phytoplankton. Cambridge University Press, Cambridge.

Reynolds, C. S. (2006). The ecology of phytoplankton. Cambridge University Press. 
Reynolds, C. S., Irish, A. E., and Elliott, J. A. (2001). "The ecological basis for simulating phytoplankton responses to environmental change (PROTECH)." Ecological Modelling, 140(3), 271-291.

Reynolds, C. S., Oliver, R. L., and Walsby, A. E. (1987). “Cyanobacterial dominance: The role of buoyancy regulation in dynamic lake environments." New Zealand Journal of Marine and Freshwater Research, 21(3), 379-390.

Richards, S. A., Possingham, H. P., and Noye, J. (1996). "Diel vertical migration: modelling light-mediated mechanisms." Journal of Plankton Research, 18(12), 2199-2222.

Ringelberg, J. (1999). "The photobehaviour of Daphnia spp. as a model to explain diel vertical migration in zooplankton." Biological Reviews, 74, 397-423.

Ringelberg, J. (2010). Diel Vertical Migration of Zooplankton in Lakes and Oceans. Springer Netherlands, Dordrecht.

Ringelberg, J., and Van Gool, E. (2003). "On the combined analysis of proximate and ultimate aspects in diel vertical migration (DVM) research." Hydrobiologia, 491(1-3), 85-90.

Rinke, K., and Petzoldt, T. (2008). "Individual-based simulation of diel vertical migration of Daphnia: A synthesis of proximate and ultimate factors." Limnologica, 38(3-4), 269-285.

Rowe, M. D., Anderson, E. J., Wynne, T. T., Stumpf, R. P., Fanslow, D. L., Kijanka, K., Vanderploeg, H. A., Strickler, J. R., and Davis, T. W. (2016). "Vertical distribution of buoyant Microcystis blooms in a Lagrangian particle tracking model for short-term forecasts in Lake Erie." Journal of Geophysical Research: Oceans, 121(7), 5296-5314.

Semyalo, R., Nattabi, J. K., and Larsson, P. (2009). “Diel Vertical Migration of zooplankton in a eutrophic bay of Lake Victoria." Hydrobiologia, 635(1), 383394.

Serizawa, H., Amemiya, T., Rossberg, A. G., and Itoh, K. (2008). “Computer simulations of seasonal outbreak and diurnal vertical migration of cyanobacteria." Limnology, 9(3), 185-194.

Simoncelli, S., Thackeray, S. J., and Wain, D. J. (2017). "Can small zooplankton mix lakes?" Limnology and Oceanography Letters, 2(5), 167-176. 
Tarling, G., Burrows, M., Matthews, J., Saborowski, R., Buchholz, F., Bedo, A., and Mayzaud, P. (2000). "An optimisation model of the diel vertical migration of northern krill (Meganyctiphanes norvegica) in the Clyde Sea and the Kattegat." $57,13$.

Trolle, D., Hamilton, D. P., Hipsey, M. R., Bolding, K., Bruggeman, J., Mooij, W. M., Janse, J. H., Nielsen, A., Jeppesen, E., Elliott, J. A., Makler-Pick, V., Petzoldt, T., Rinke, K., Flindt, M. R., Arhonditsis, G. B., Gal, G., Bjerring, R., Tominaga, K., Hoen, J., Downing, A. S., Marques, D. M., Fragoso, C. R., Søndergaard, M., and Hanson, P. C. (2012). "A community-based framework for aquatic ecosystem models." Hydrobiologia, 683(1), 25-34.

Van Gool, E., and Ringelberg, J. (2003). "What goes down must come up: symmetry in light-induced migration behaviour of Daphnia." Hydrobiologia, 491(1-3), 301-307.

Visser, P. M., Ibelings, B. W., Bormans, M., and Huisman, J. (2016). "Artificial mixing to control cyanobacterial blooms: A review." Aquatic Ecology, 50(3), 423-441.

Visser, P. M., Passarge, J., and Mur, L. R. (1997). "Modelling vertical migration of the cyanobacterium Microcystis." Hydrobiologia, 349, 99-109.

Wallace, B. B., and Hamilton, D. P. (2000). "Simulation of water-bloom formation in the cyanobacterium Microcystis aeruginosa." Journal of Plankton Research, 22(6), 1127-1138.

Wallace, B. B., Bailey, M. C., and Hamilton, D. P. (2000). "Simulation of vertical position of buoyancy regulating Microcystis aeruginosa in a shallow eutrophic lake:" Aquatic Sciences, 62(4), 320-333.

Wallace, B. B., and Hamilton, D. P. (1999). "The effect of variations in irradiance on buoyancy regulation in Microcystis aeruginosa." Limnology and Oceanography, $44(2), 273-281$.

Walsby, A. E. (2005). "Stratification by cyanobacteria in lakes: A dynamic buoyancy model indicates size limitations met by Planktothrix rubescens filaments." New Phytologist, 168(2), 365-376.

Wang, C., Feng, T., Wang, P., Hou, J., and Qian, J. (2017). “Understanding the transport feature of bloom-forming Microcystis in a large shallow lake: A new combined hydrodynamic and spatially explicit agent-based modelling approach." Ecological Modelling, 343, 25-38. 
Wang, L., Cai, Q., Zhang, M., Xu, Y., Kong, L., and Tan, L. (2011). “Vertical distribution patterns of phytoplankton in summer Microcystis bloom period of Xiangxi Bay, Three Gorges Reservoir, China." Fresenius Environmental Bulletin, $20(3), 9$.

Wilhelmus, M. M., and Dabiri, J. O. (2014). “Observations of large-scale fluid transport by laser-guided plankton aggregations." Physics of Fluids, 26(10), 101302.

Williamson, C. E., Fischer, J. M., Bollens, S. M., Overholt, E. P., and Breckenridge, J. K. (2011). "Toward a more comprehensive theory of zooplankton diel vertical migration: Integrating ultraviolet radiation and water transparency into the biotic paradigm." Limnology and Oceanography, 56(5), 1603-1623.

Wu, Z., Shi, J., and Li, R. (2009). “Comparative studies on photosynthesis and phosphate metabolism of Cylindrospermopsis raciborskii with Microcystis aeruginosa and Aphanizomenon flos-aquae." Harmful Algae, 8(6), 910-915.

Wu, Z., and Song, L. (2008). "Physiological comparison between colonial and unicellular forms of Microcystis aeruginosa Kütz. (cyanobacteria)." Phycologia, $47(1), 98-104$.

Yao, B., Liu, Q., Gao, Y., and Cao, Z. (2017). "Characterizing vertical migration of Microcystis aeruginosa and conditions for algal bloom development based on a light-driven migration model." Ecological Research, 32(6), 961-969.

Zhang, M., Shi, X., Yu, Y., and Kong, F. (2011). “The acclimative changes in photochemistry after colony formation of the cyanobacteria Microcystis aeruginosa 1: Photoadaptive changes of M. Aeruginosa." Journal of Phycology, $47(3), 524-532$.

Zhao, H., Zhu, W., Chen, H., Zhou, X., Wang, R., and Li, M. (2016). "Numerical simulation of the vertical migration of Microcystis (cyanobacteria) colonies based on turbulence drag." Journal of Limnology.

Zhu, W., Feng, G., Chen, H., Wang, R., Tan, Y., and Zhao, H. (2018). "Modelling the vertical migration of different-sized Microcystis colonies: coupling turbulent mixing and buoyancy regulation." Environmental Science and Pollution Research, 25(30), 30339-30347.

Zhu, W., Li, M., Luo, Y., Dai, X., Guo, L., Xiao, M., Huang, J., and Tan, X. (2014). "Vertical distribution of Microcystis colony size in Lake Taihu: Its role in algal blooms." Journal of Great Lakes Research, 40(4), 949-955. 


\section{Appendix A: Profile Plots}
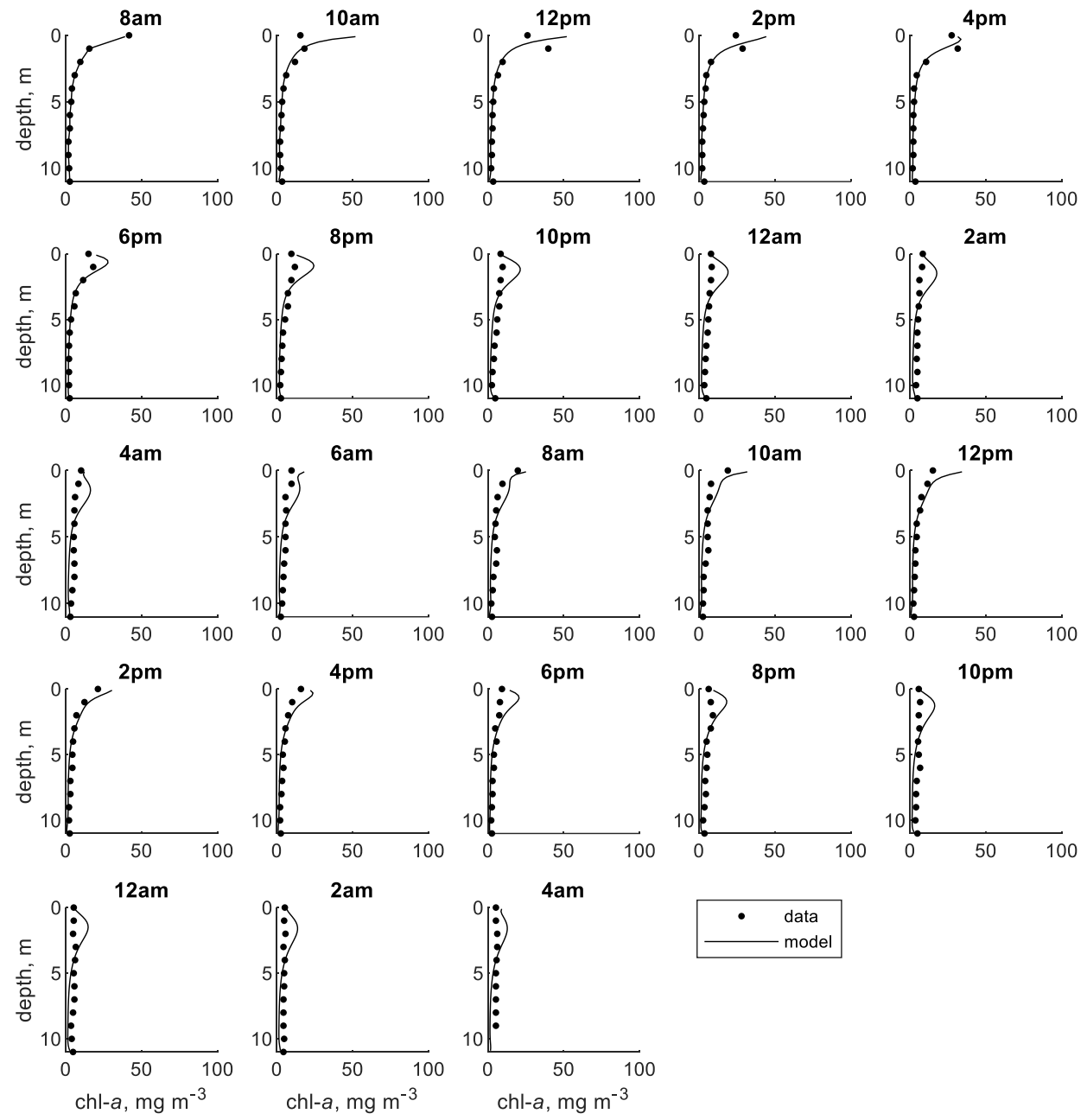

Figure A 1 Observed and predicted concentration profiles of chlorophyll a in Shennong Stream enclosure site using time-varying velocity model (continuum) 

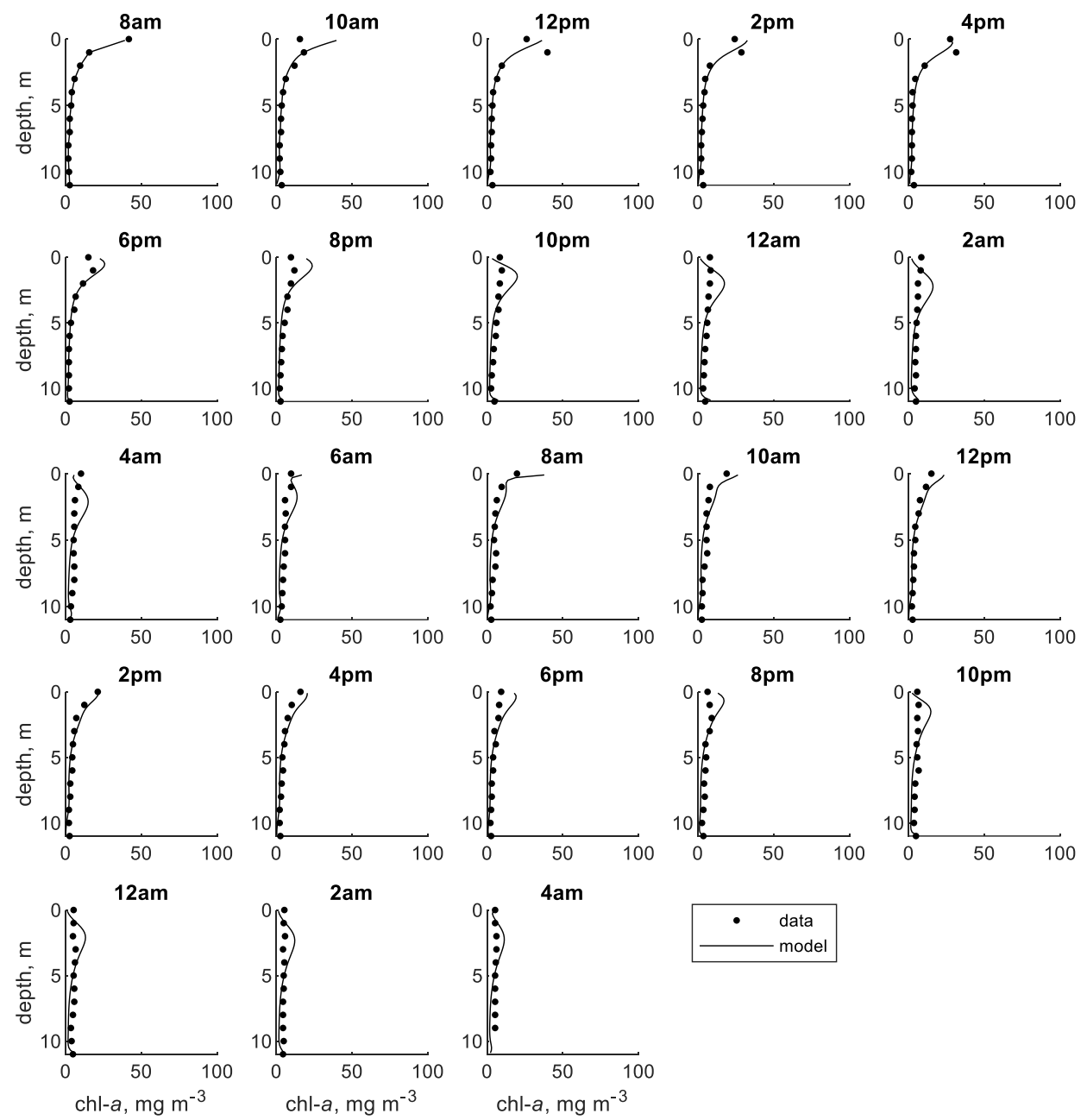

Figure A 2 Observed and predicted concentration profiles of chlorophyll a in Shennong Stream enclosure site using Belov \& Giles (1997) velocity model (continuum) 

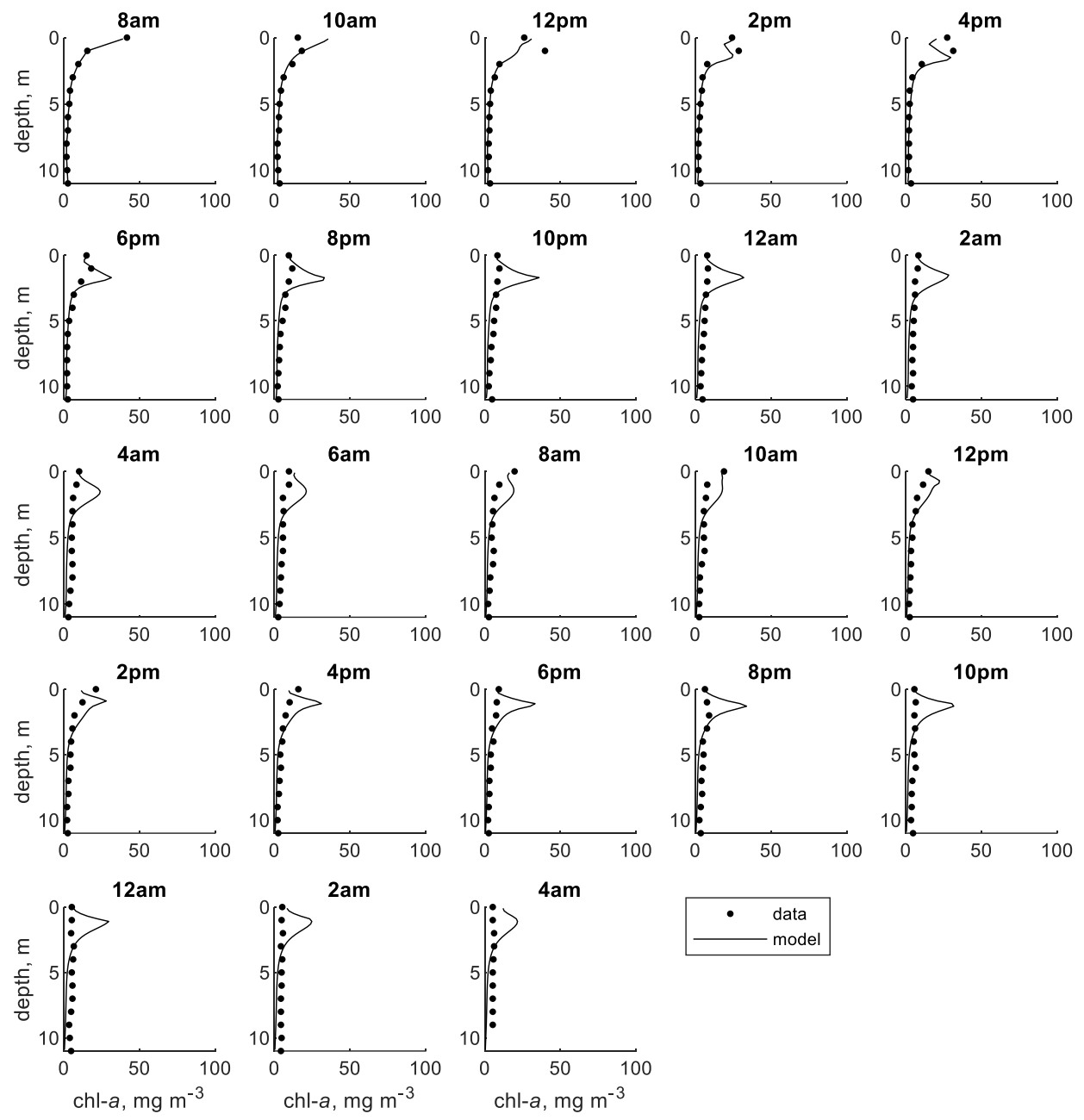

Figure A 3 Observed and predicted concentration profiles of chlorophyll a in Shennong Stream enclosure site using growth kinetics model (continuum) 

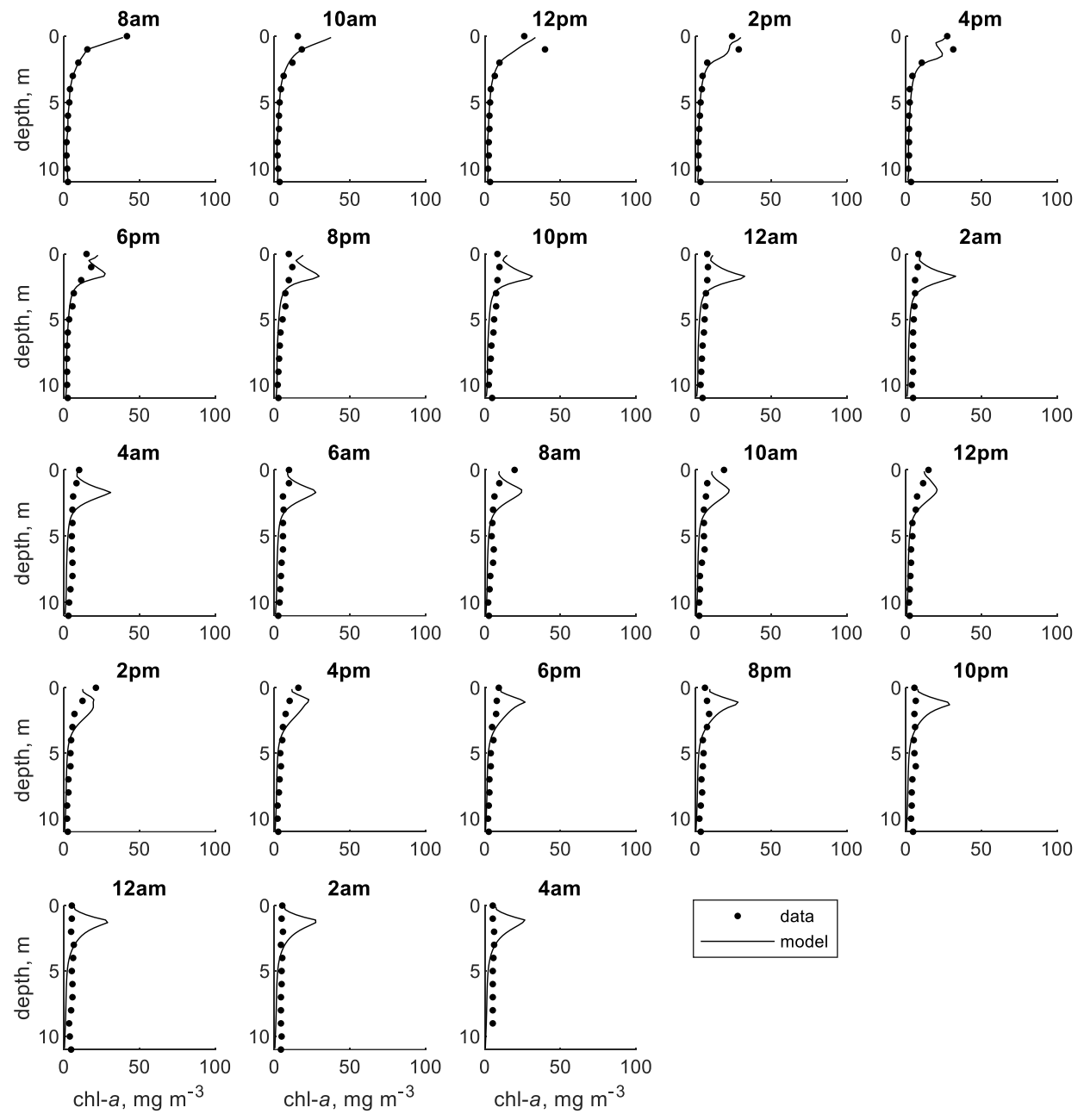

Figure A 4 Observed and predicted concentration profiles of chlorophyll a in Shennong Stream enclosure site using growth kinetics model with time decay (continuum) 

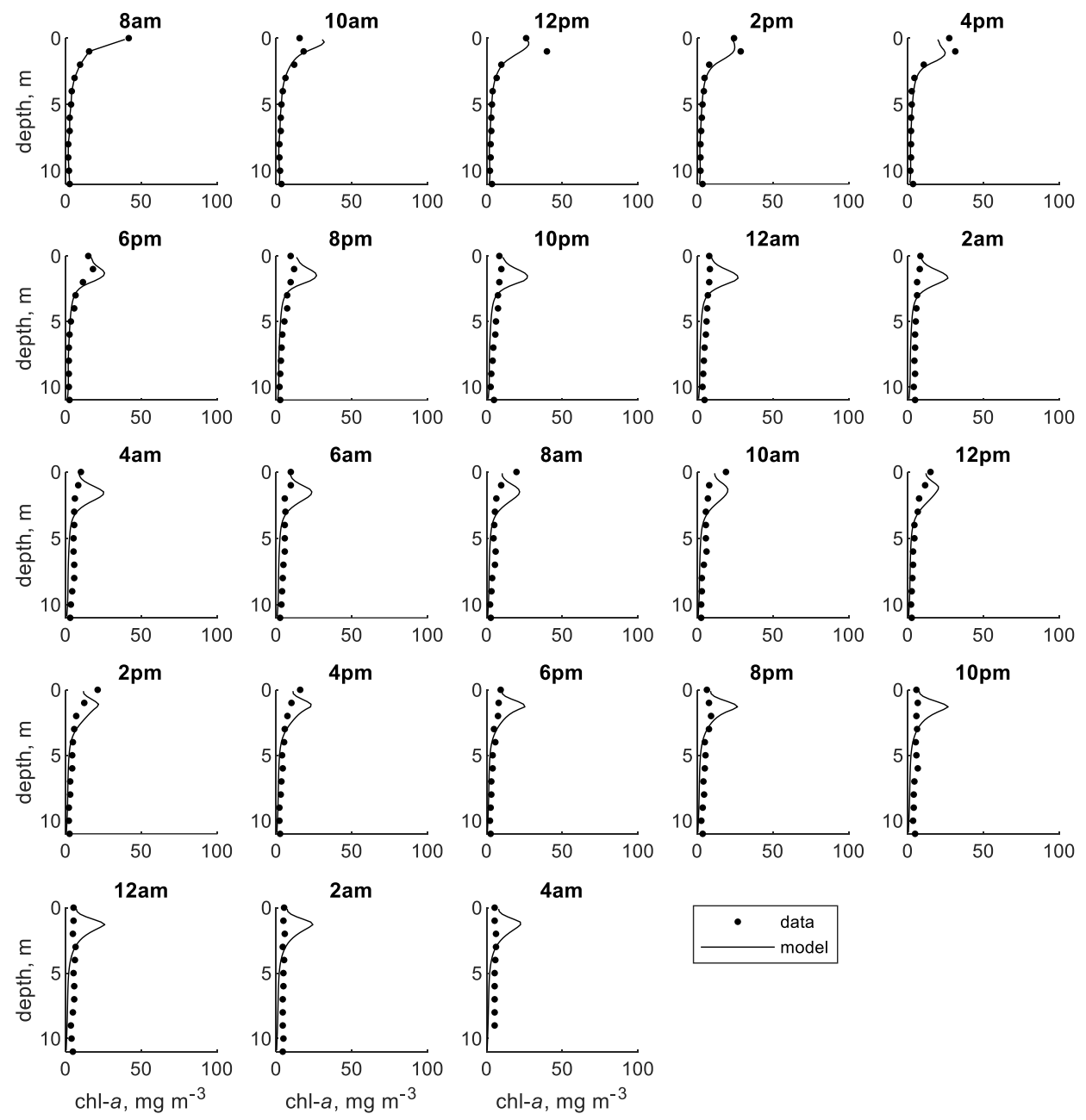

Figure A 5 Observed and predicted concentration profiles of chlorophyll a in Shennong Stream enclosure site using Visser et al. (1997) model (continuum) 

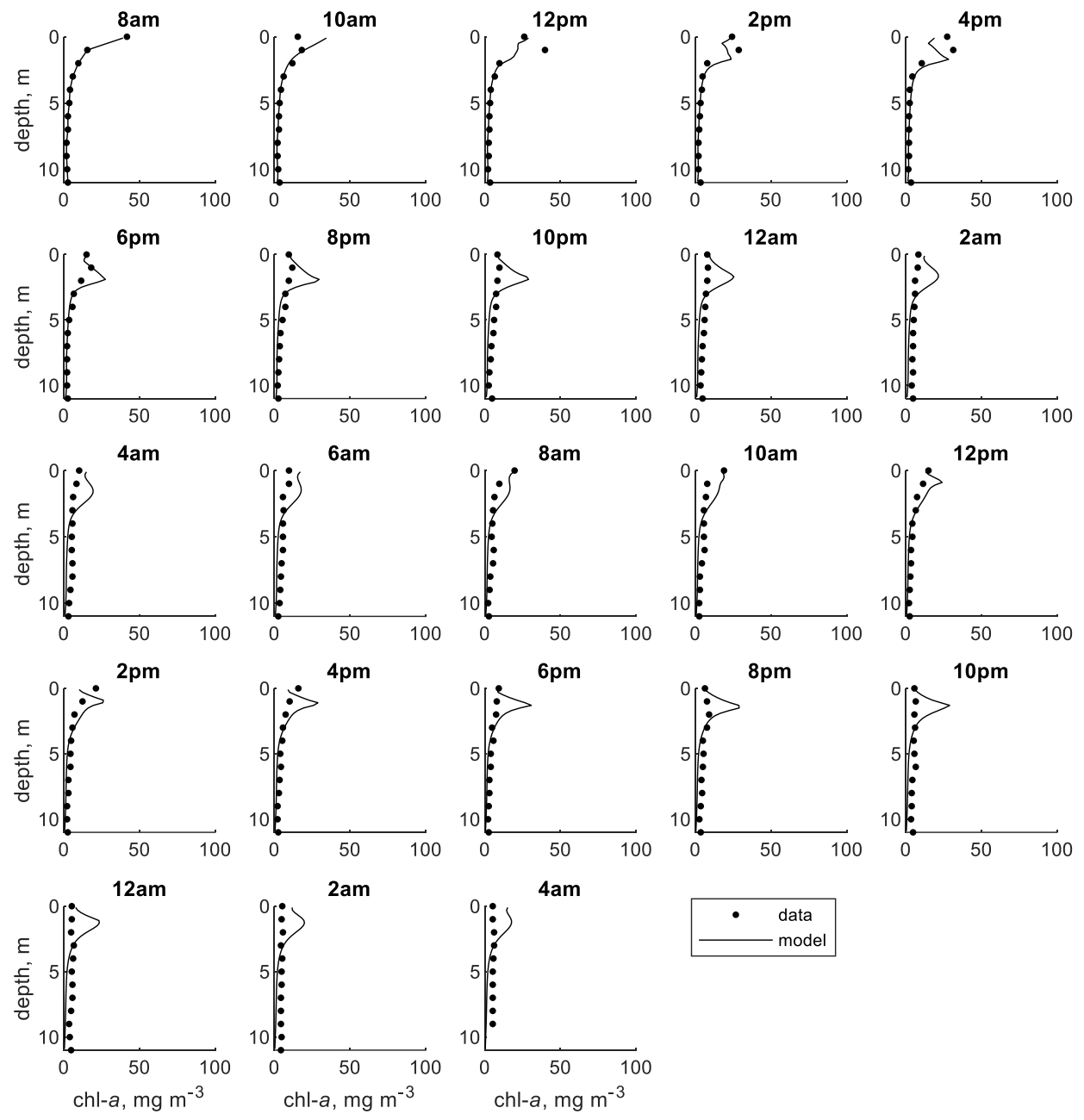

Figure A 6 Observed and predicted concentration profiles of chlorophyll a in Shennong Stream enclosure site using light function model (continuum) 

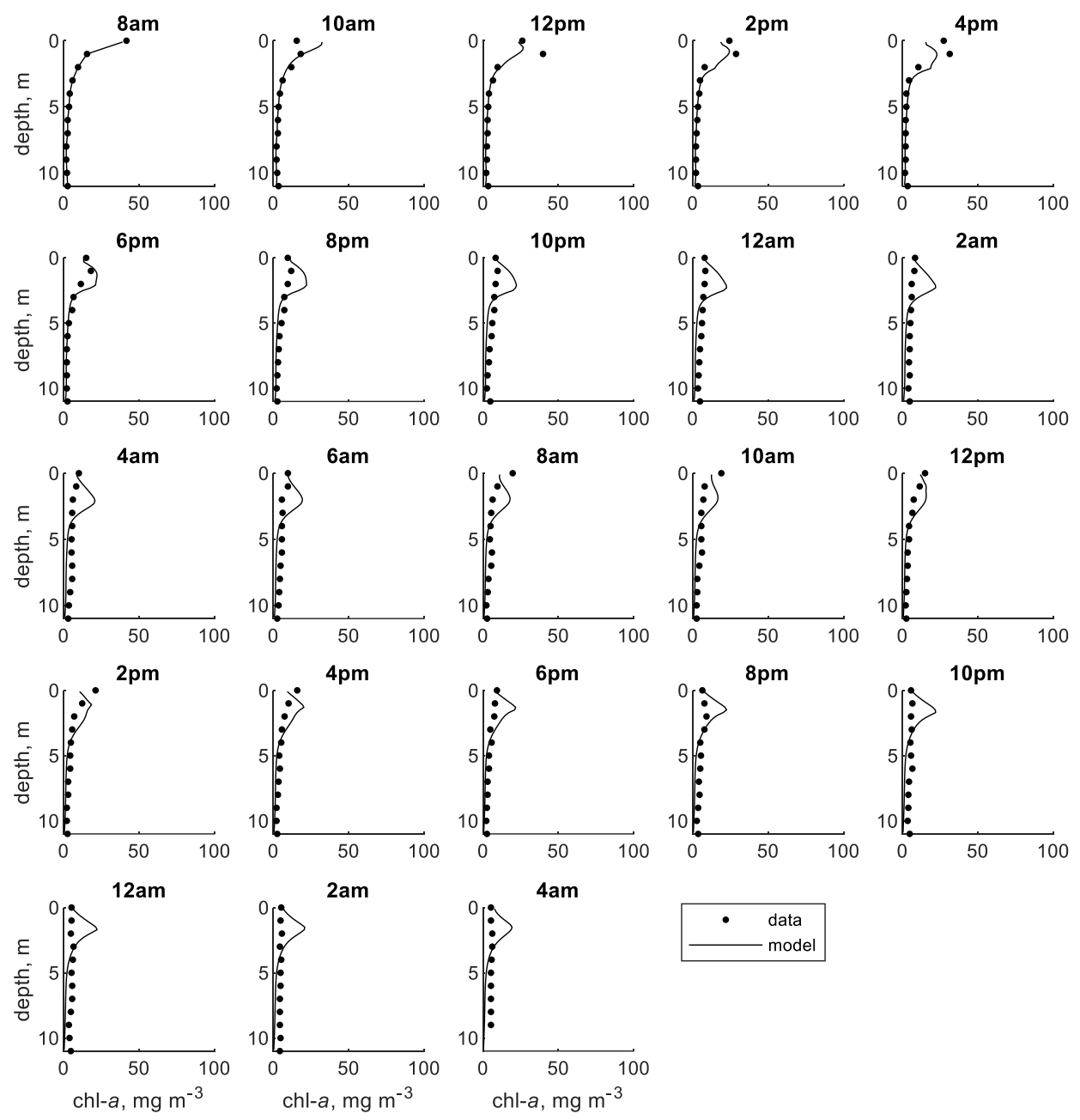

Figure A 7 Observed and predicted concentration profiles of chlorophyll a in Shennong Stream enclosure site using light function model with time decay (continuum) 

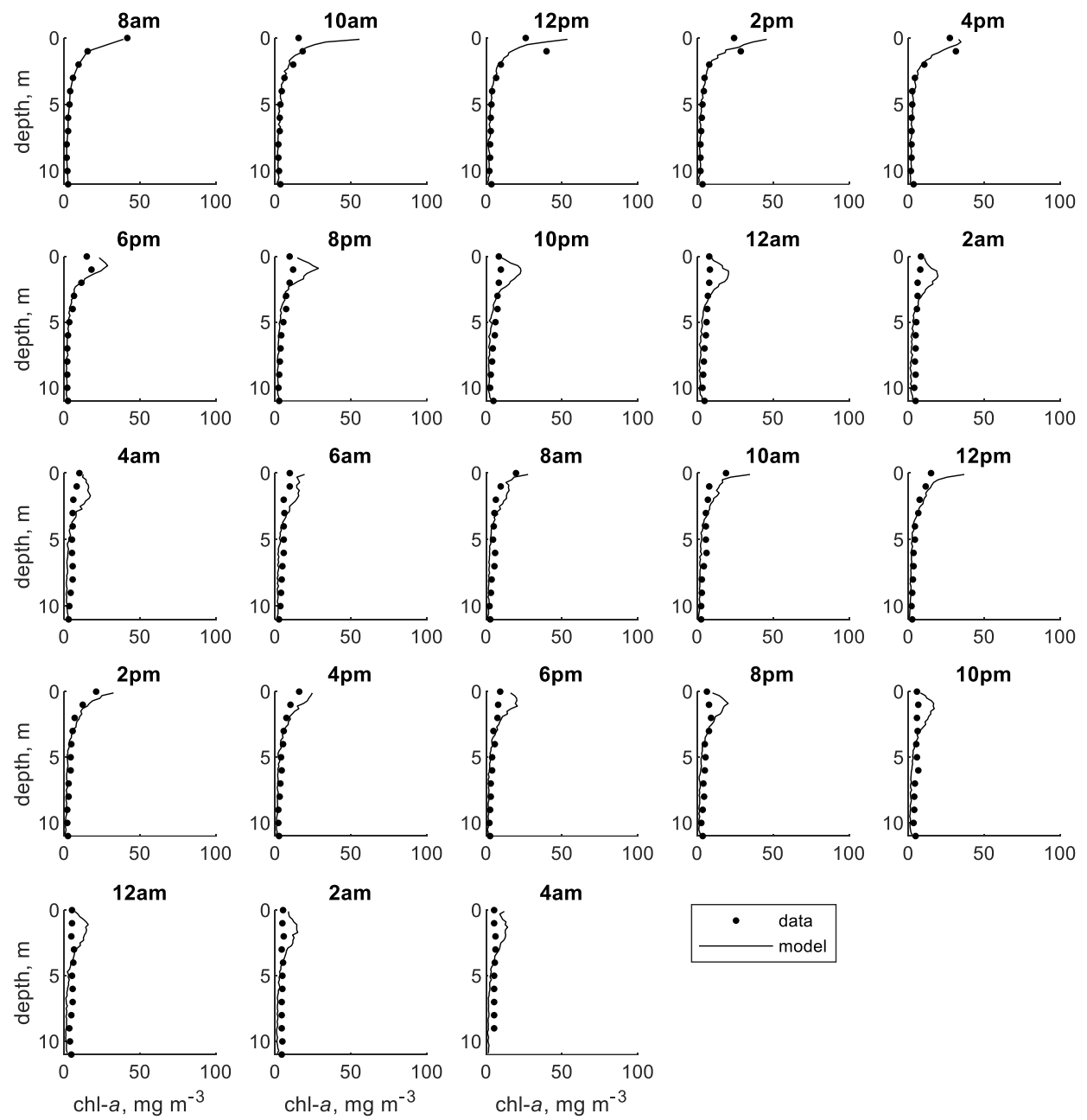

Figure A 8 Observed and predicted concentration profiles of chlorophyll a in Shennong Stream enclosure site using time-varying velocity model (particle-tracking) 

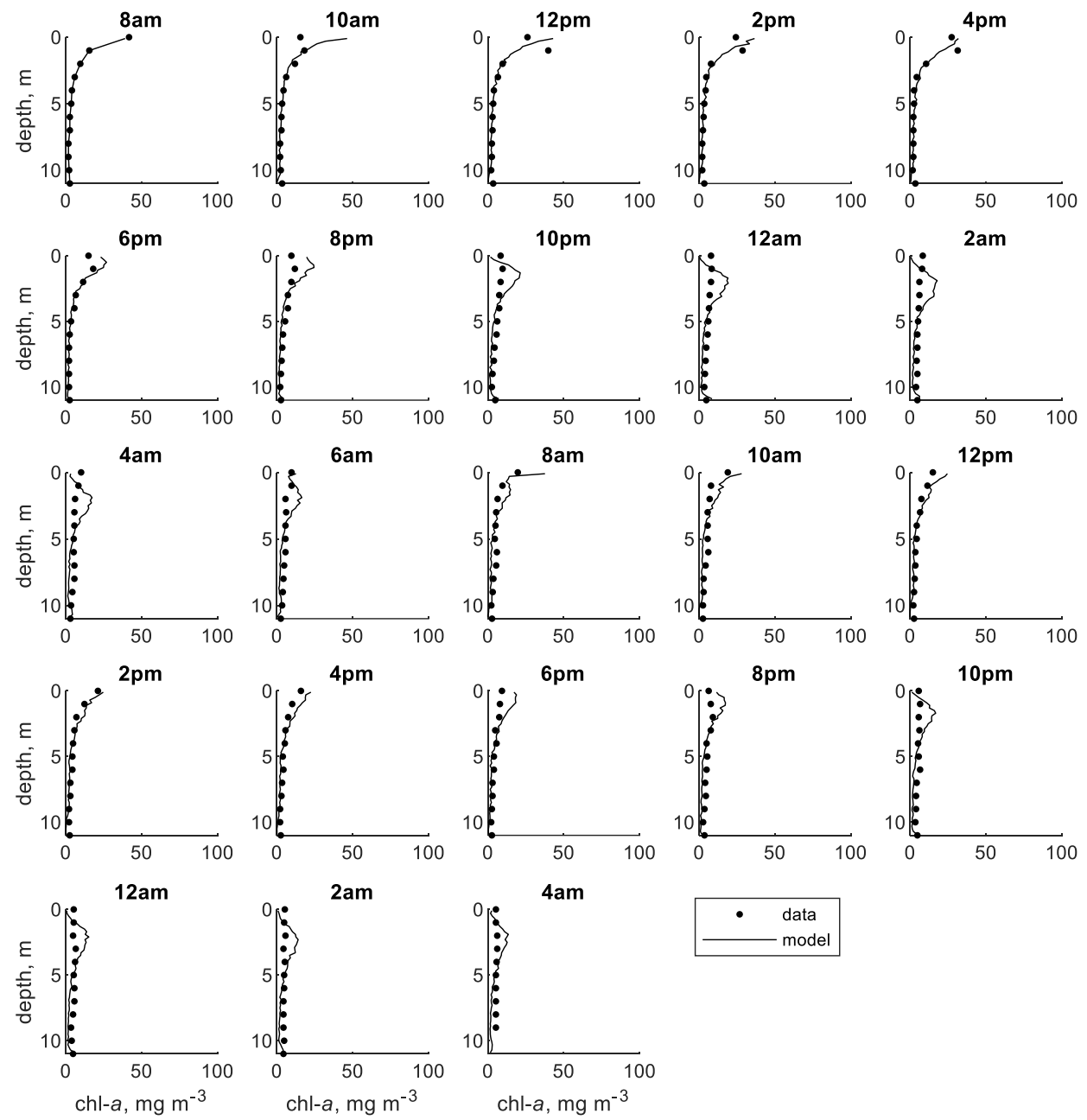

Figure A 9 Observed and predicted concentration profiles of chlorophyll a in Shennong Stream enclosure site using Belov \& Giles (1997) velocity model (particle-tracking) 

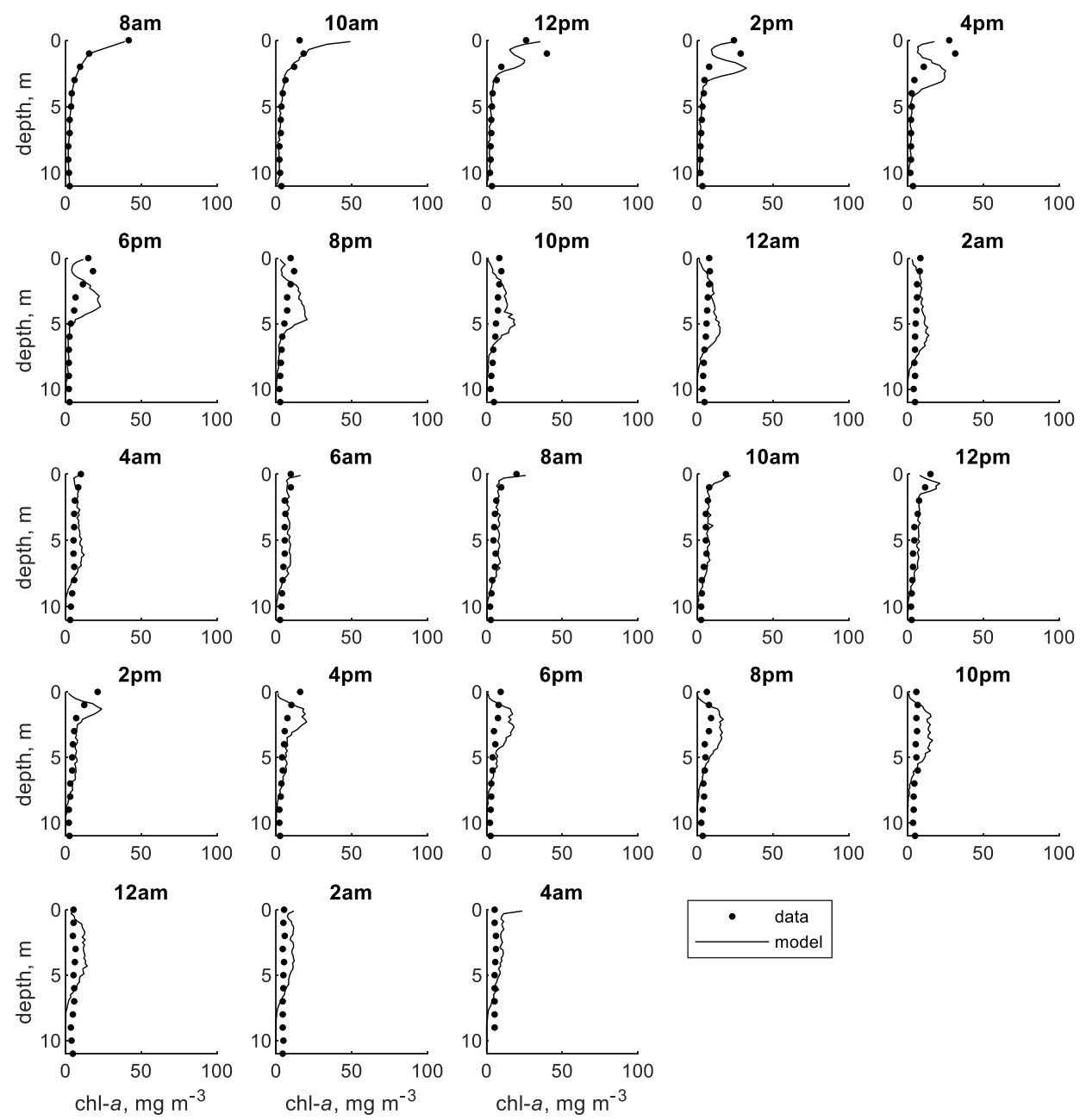

Figure A 10 Observed and predicted concentration profiles of chlorophyll a in Shennong Stream enclosure site using growth kinetics model (particle-tracking) 

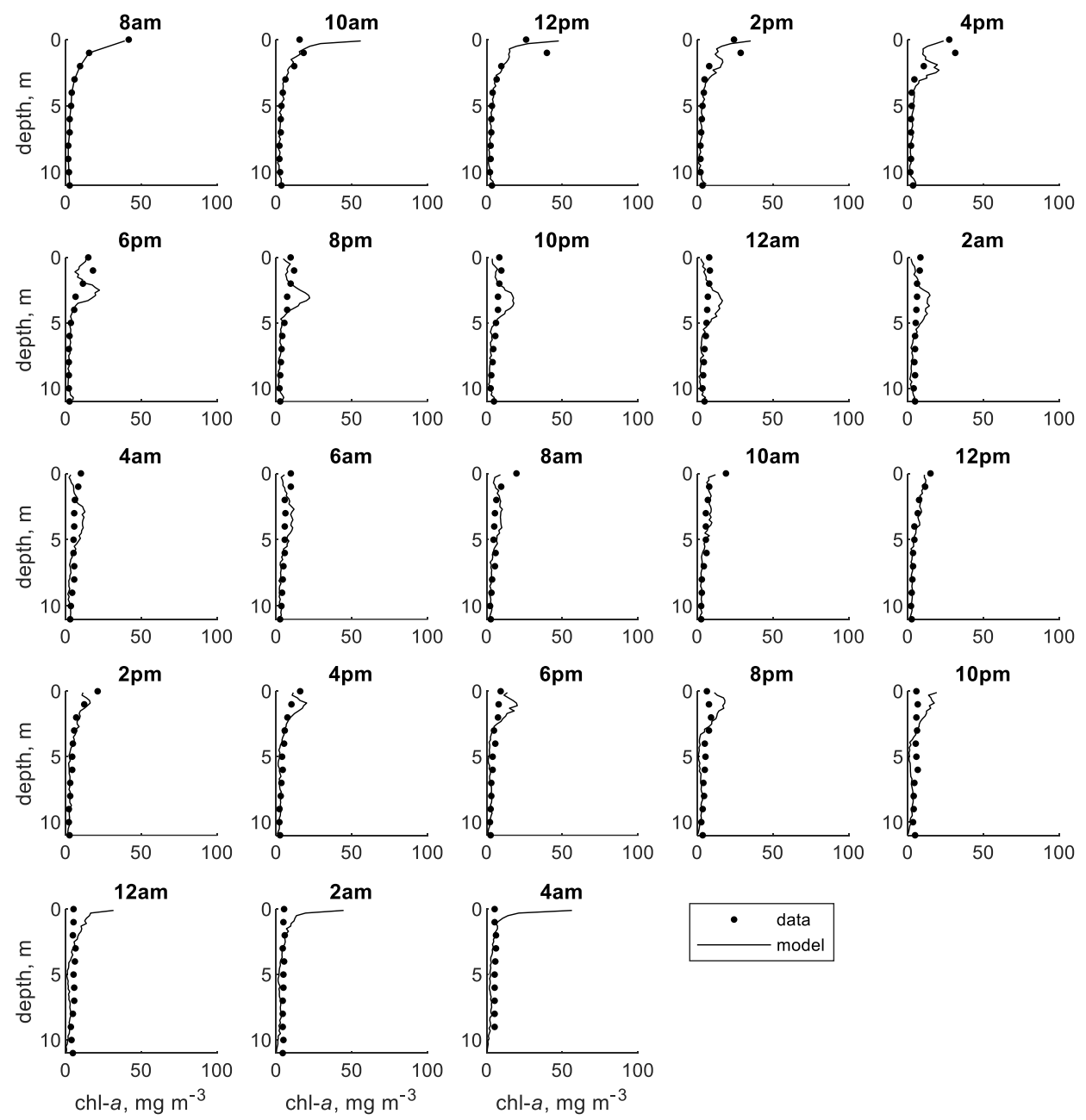

Figure A 11 Observed and predicted concentration profiles of chlorophyll a in Shennong Stream enclosure site using Visser et al. (1997)model (particle-tracking) 

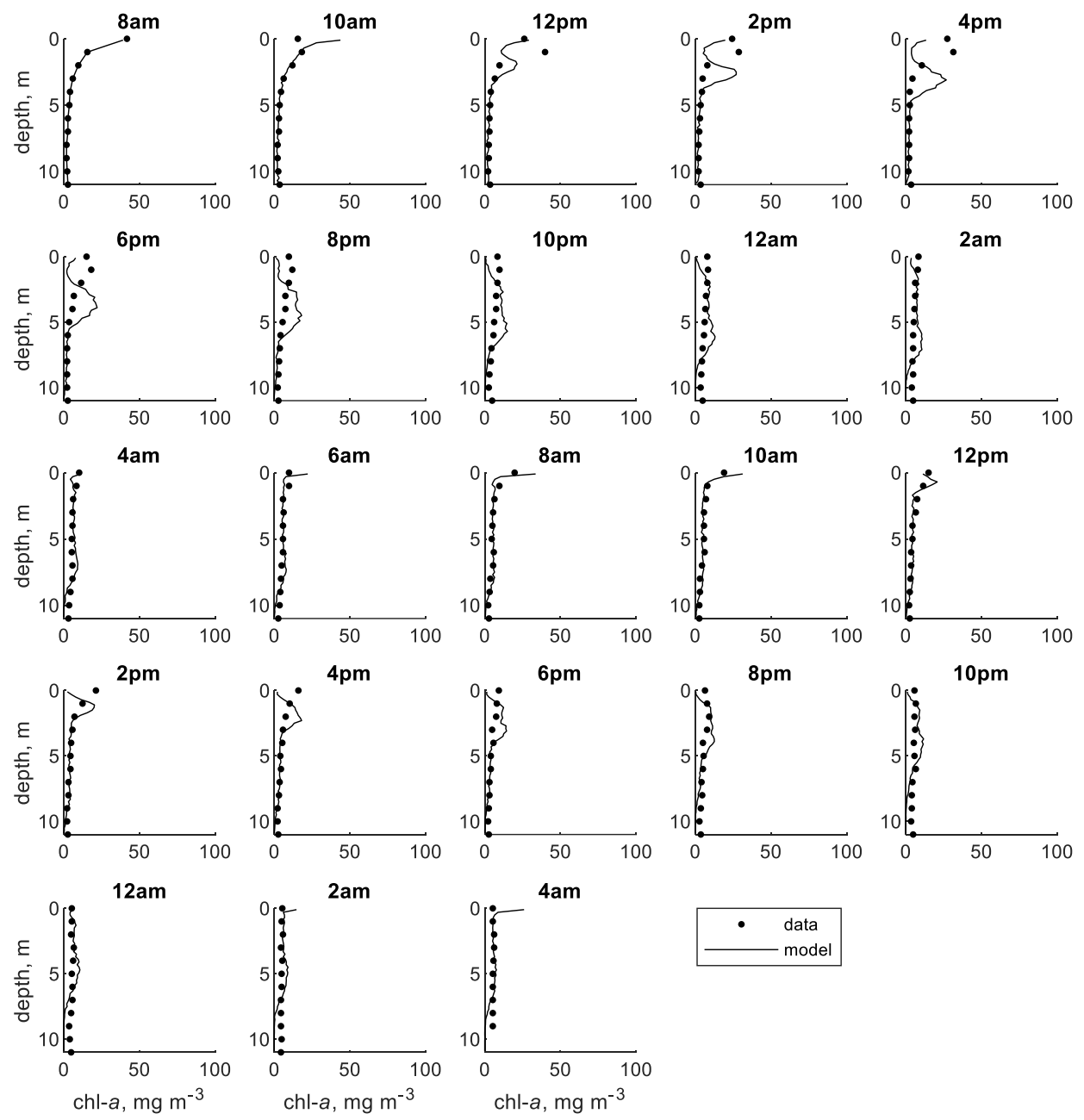

Figure A 12 Observed and predicted concentration profiles of chlorophyll a in Shennong Stream enclosure site using light function model (particle-tracking) 

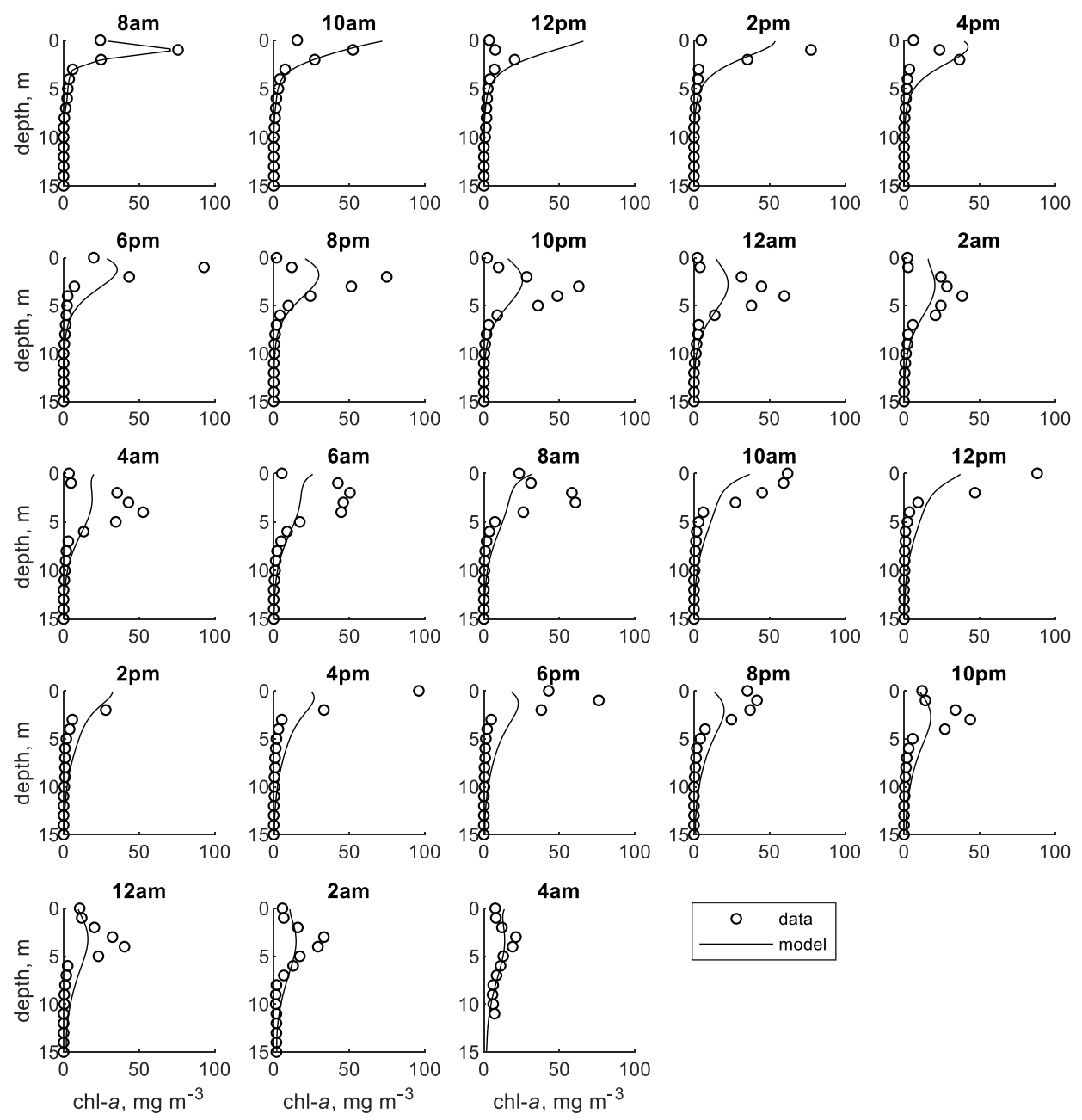

Figure A 13 Observed and predicted concentration profiles of chlorophyll a in Shennong Stream open water site using time-varying velocity model (continuum) 

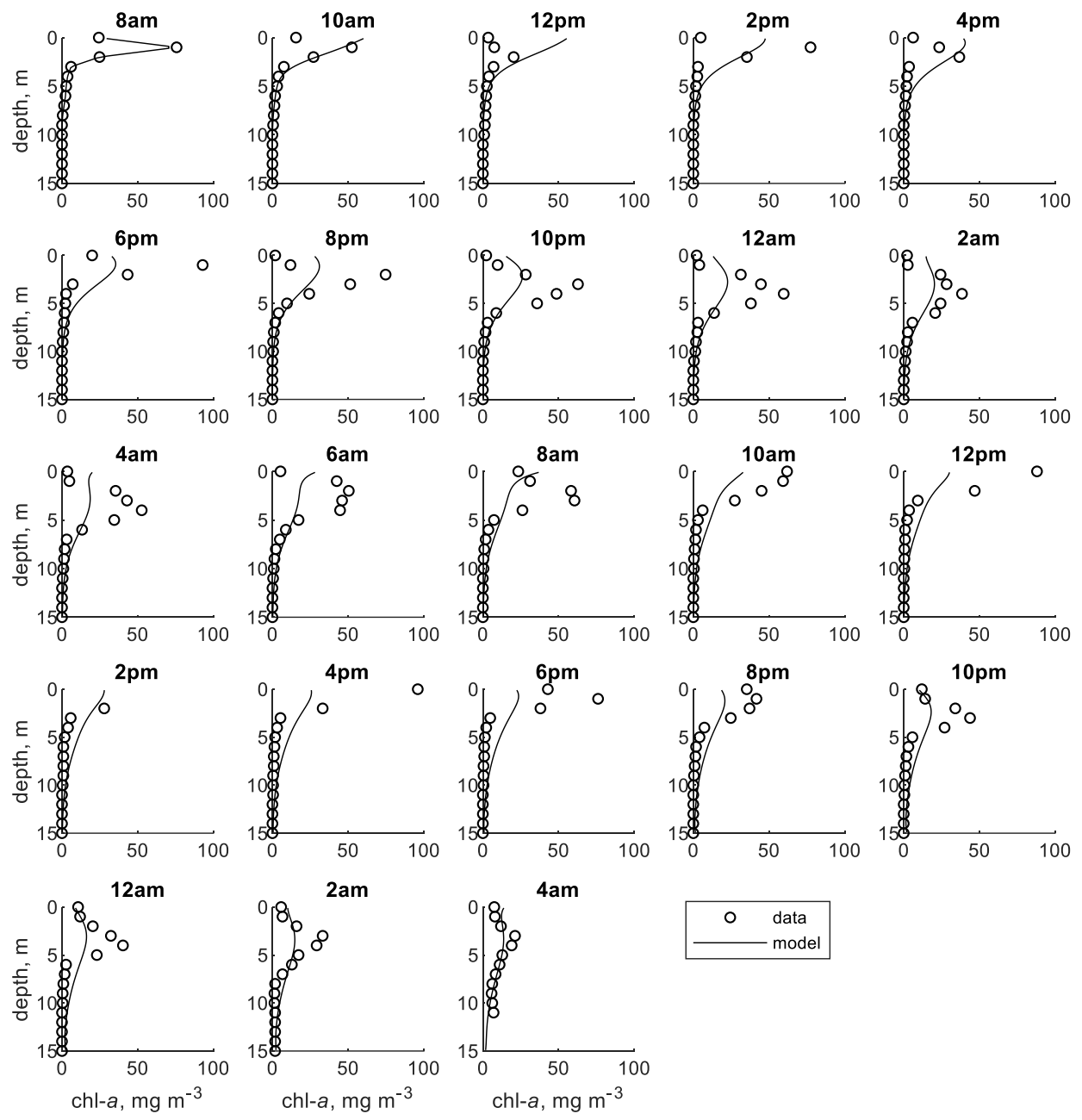

Figure A 14 Observed and predicted concentration profiles of chlorophyll a in Shennong Stream open water site using Belov \& Giles (1997) velocity model (continuum) 

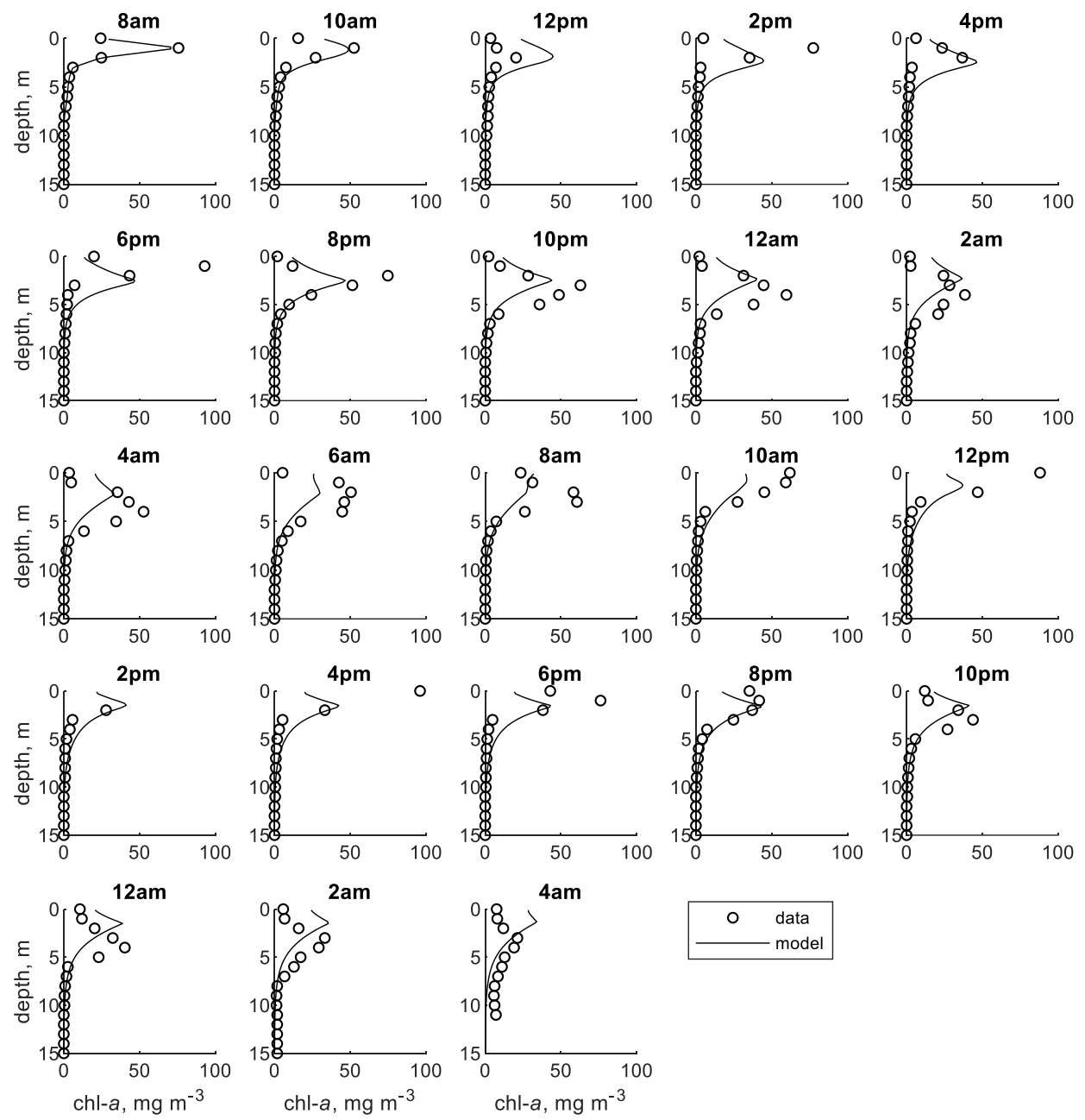

Figure A 15 Observed and predicted concentration profiles of chlorophyll a in Shennong Stream open water site using growth kinetics model without time decay (continuum) 

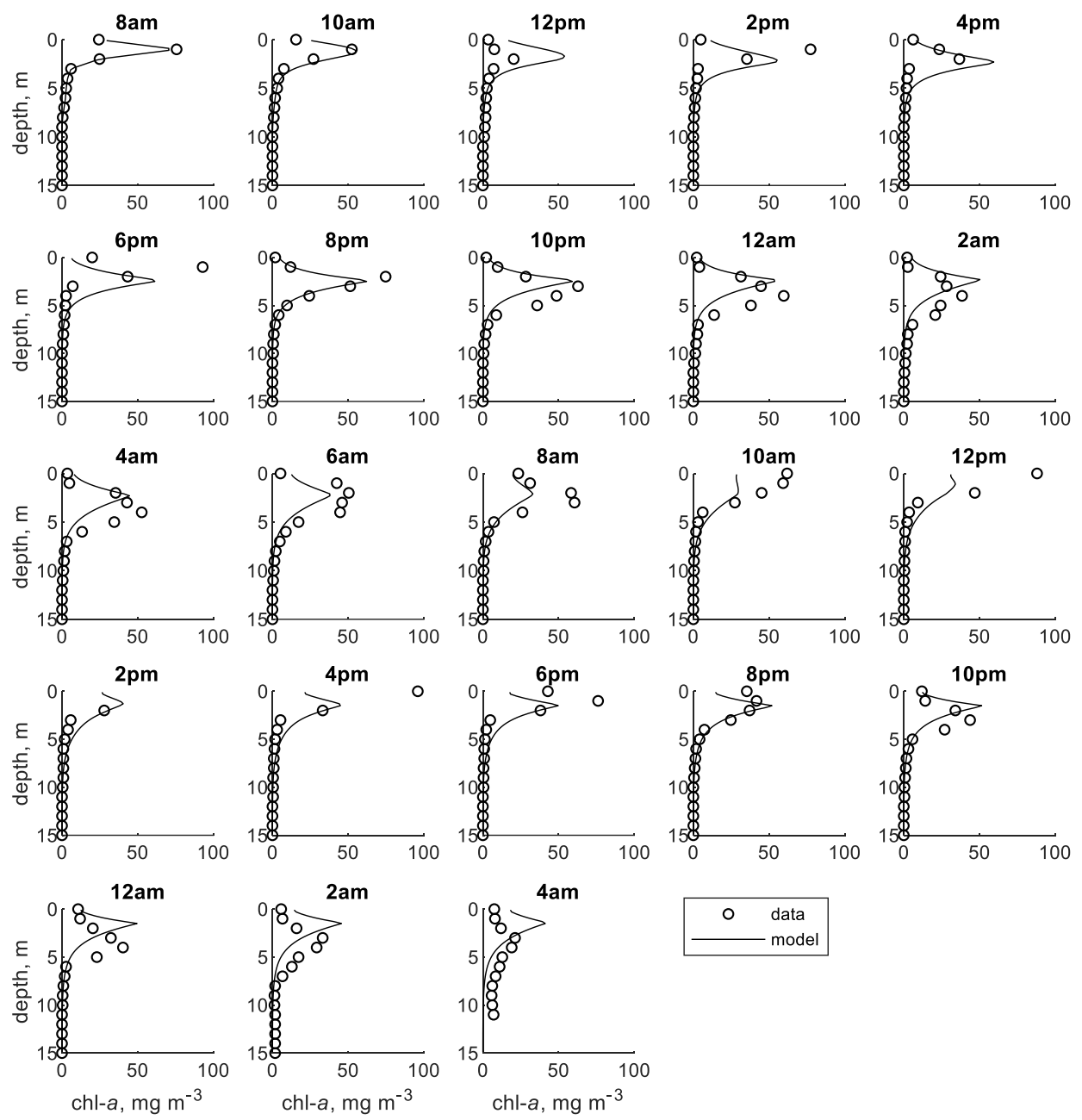

Figure A 16 Observed and predicted concentration profiles of chlorophyll a in Shennong Stream open water site using growth kinetics model with time decay (continuum) 

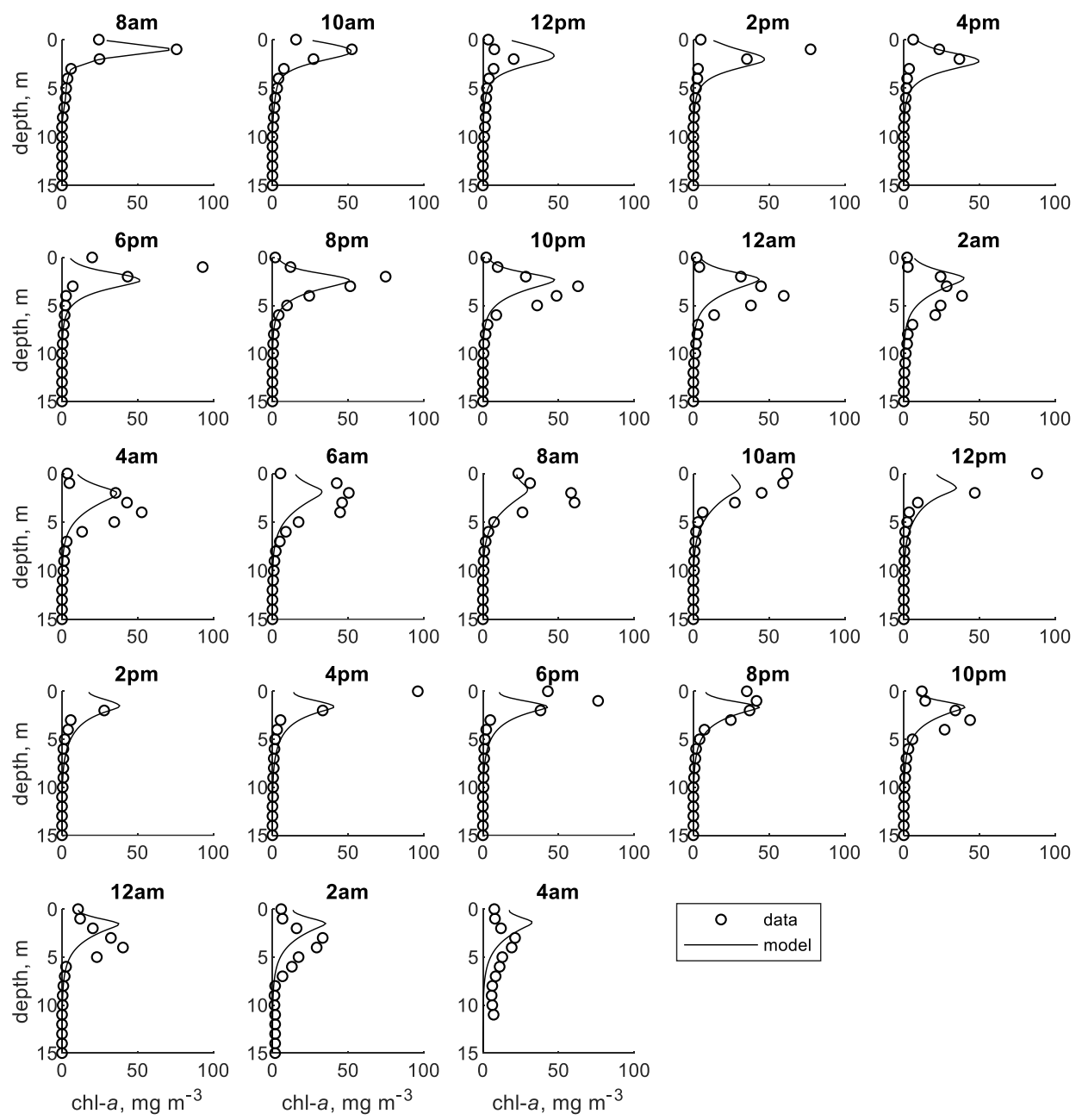

Figure A 17 Observed and predicted concentration profiles of chlorophyll a in Shennong Stream open water site using Visser et al. (!997) model (continuum) 

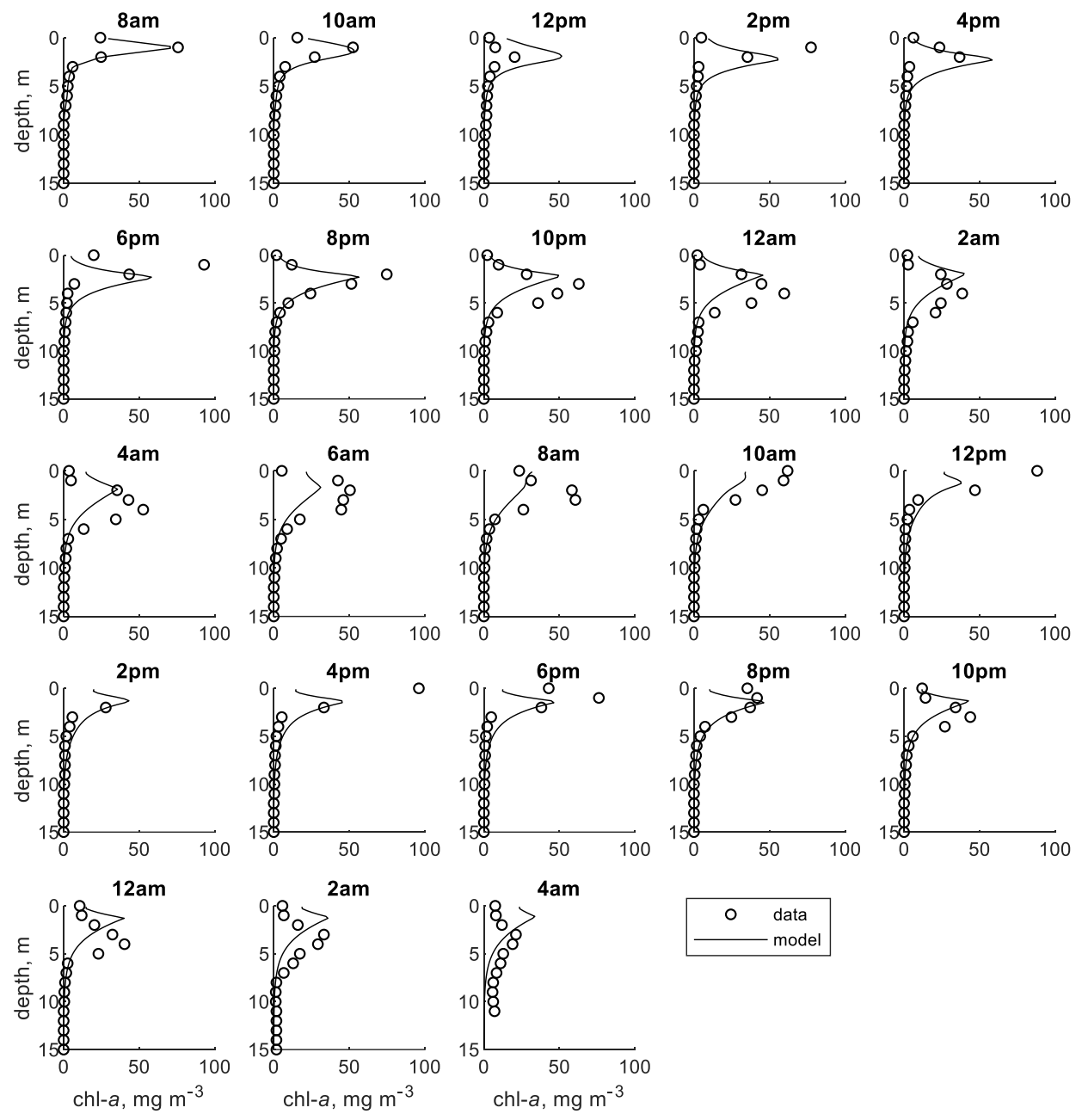

Figure A 18 Observed and predicted concentration profiles of chlorophyll a in Shennong Stream open water site using light function model without time decay (continuum) 

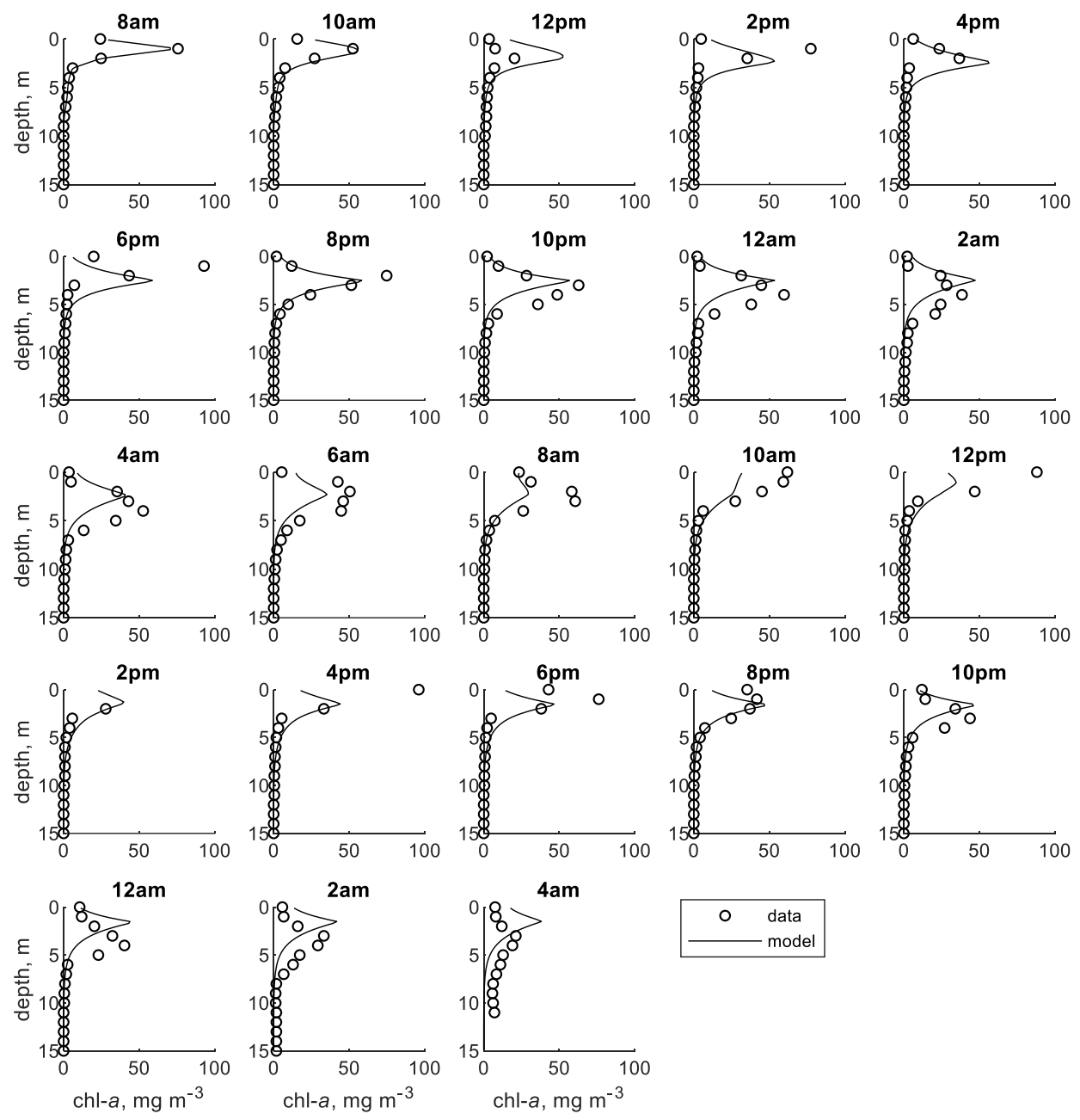

Figure A 19 Observed and predicted concentration profiles of chlorophyll a in Shennong Stream open water site using light function model with time decay (continuum) 

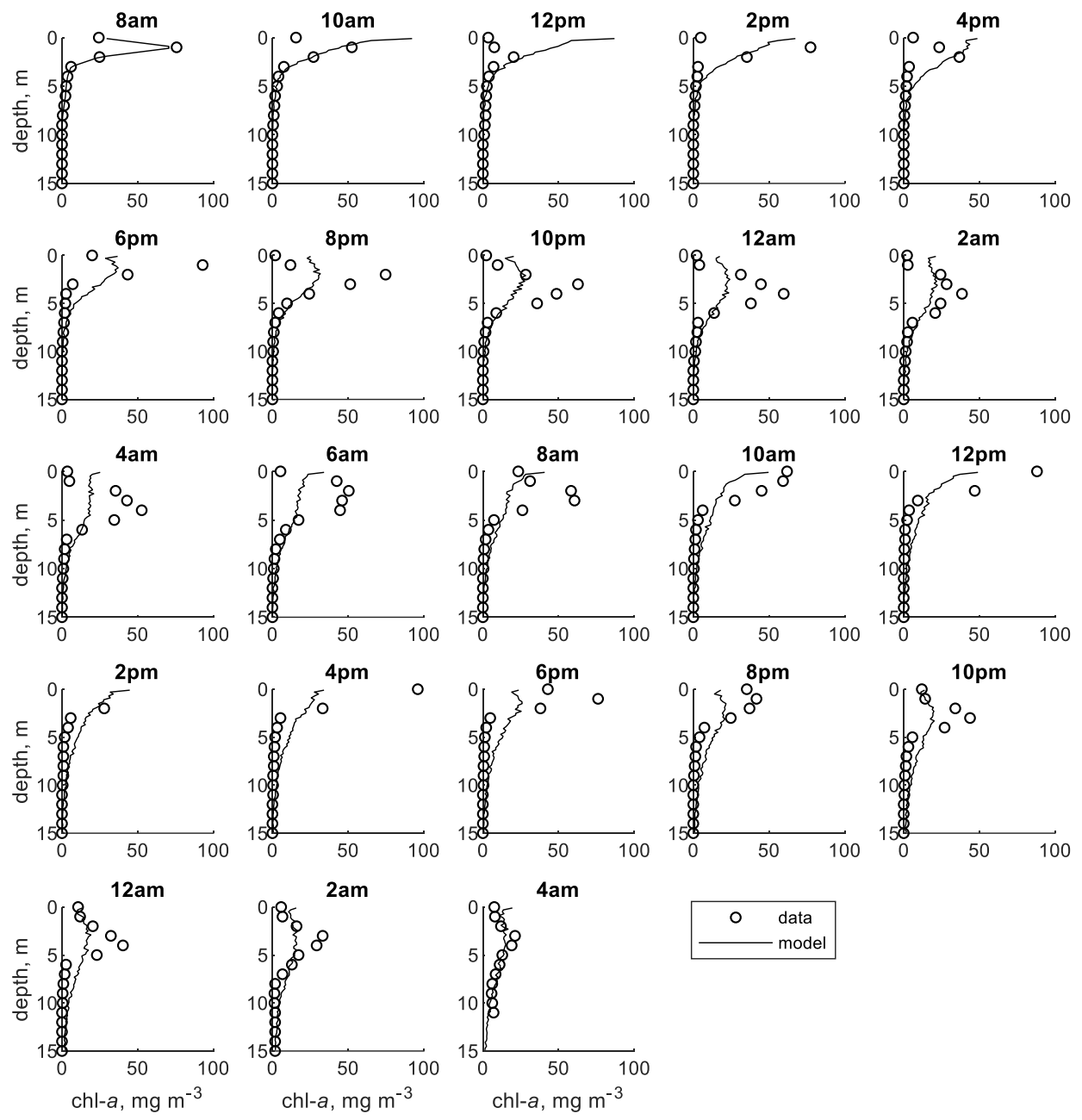

Figure A 20 Observed and predicted concentration profiles of chlorophyll a in Shennong Stream open water site using time-varying velocity model (particle-tracking) 

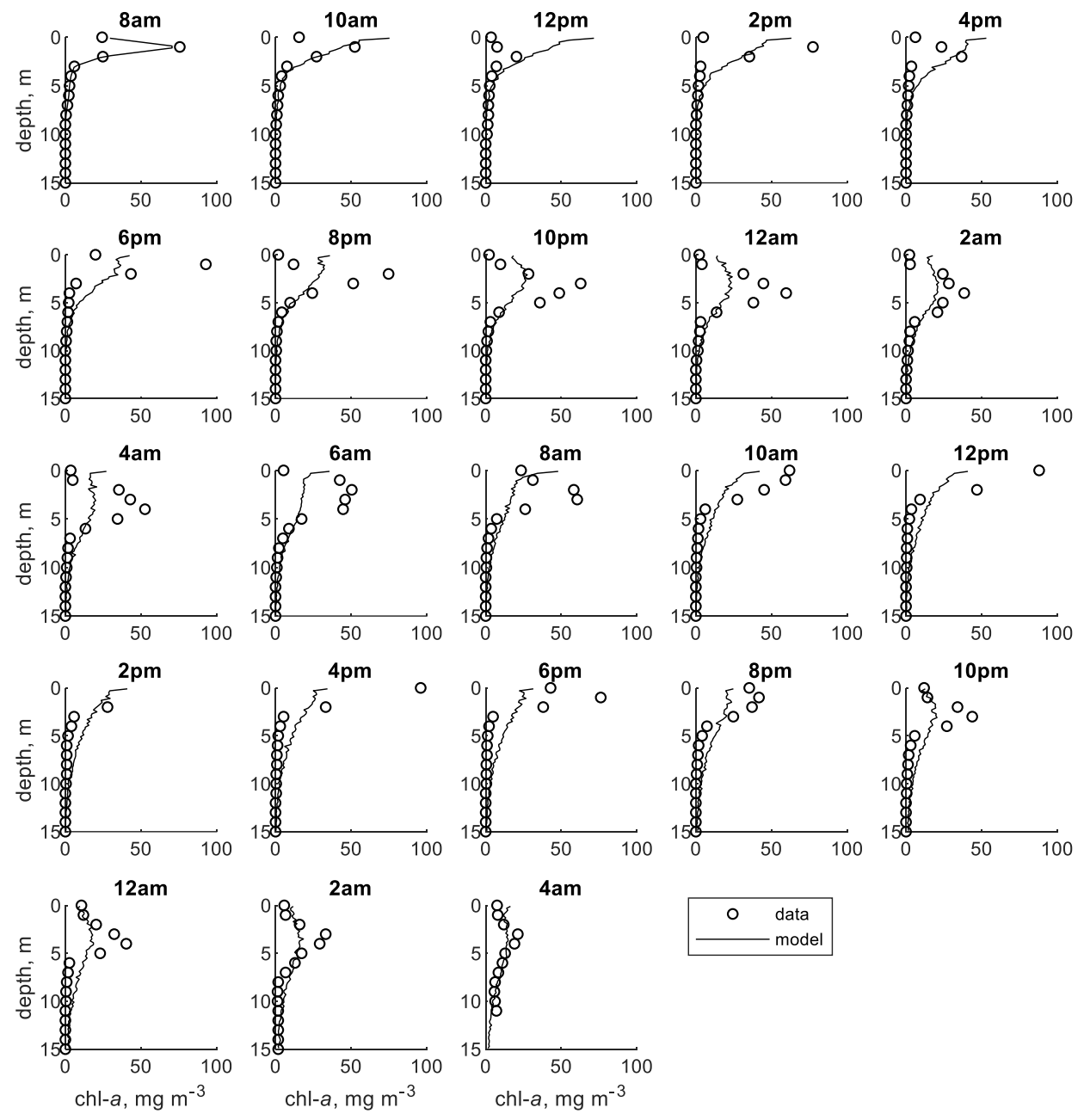

Figure A 21 Observed and predicted concentration profiles of chlorophyll a in Shennong Stream open water site using Belov \& Giles (1997) velocity model (particle-tracking) 

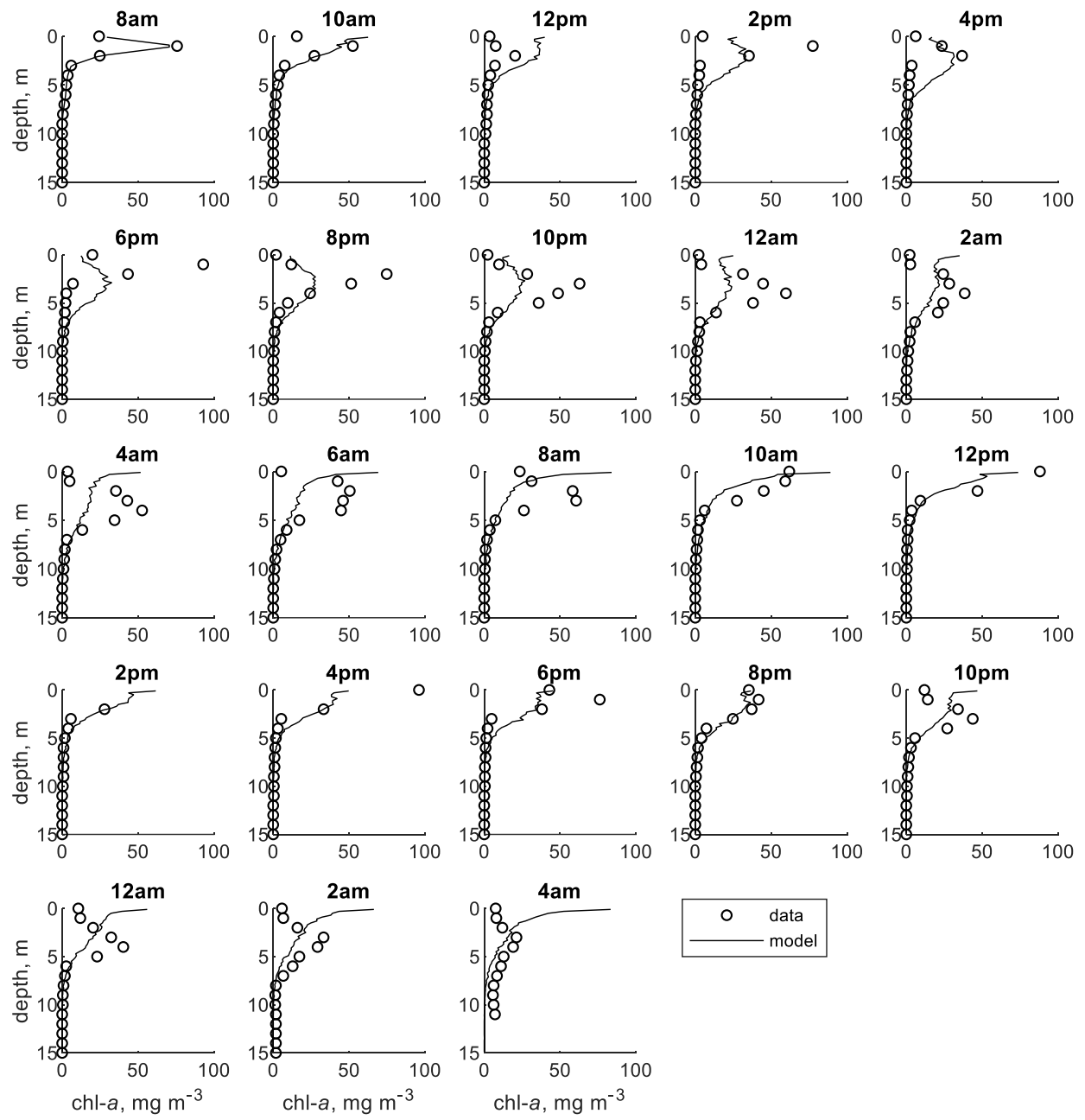

Figure A 22 Observed and predicted concentration profiles of chlorophyll a in Shennong Stream open water site using growth kinetics model (particle-tracking) 

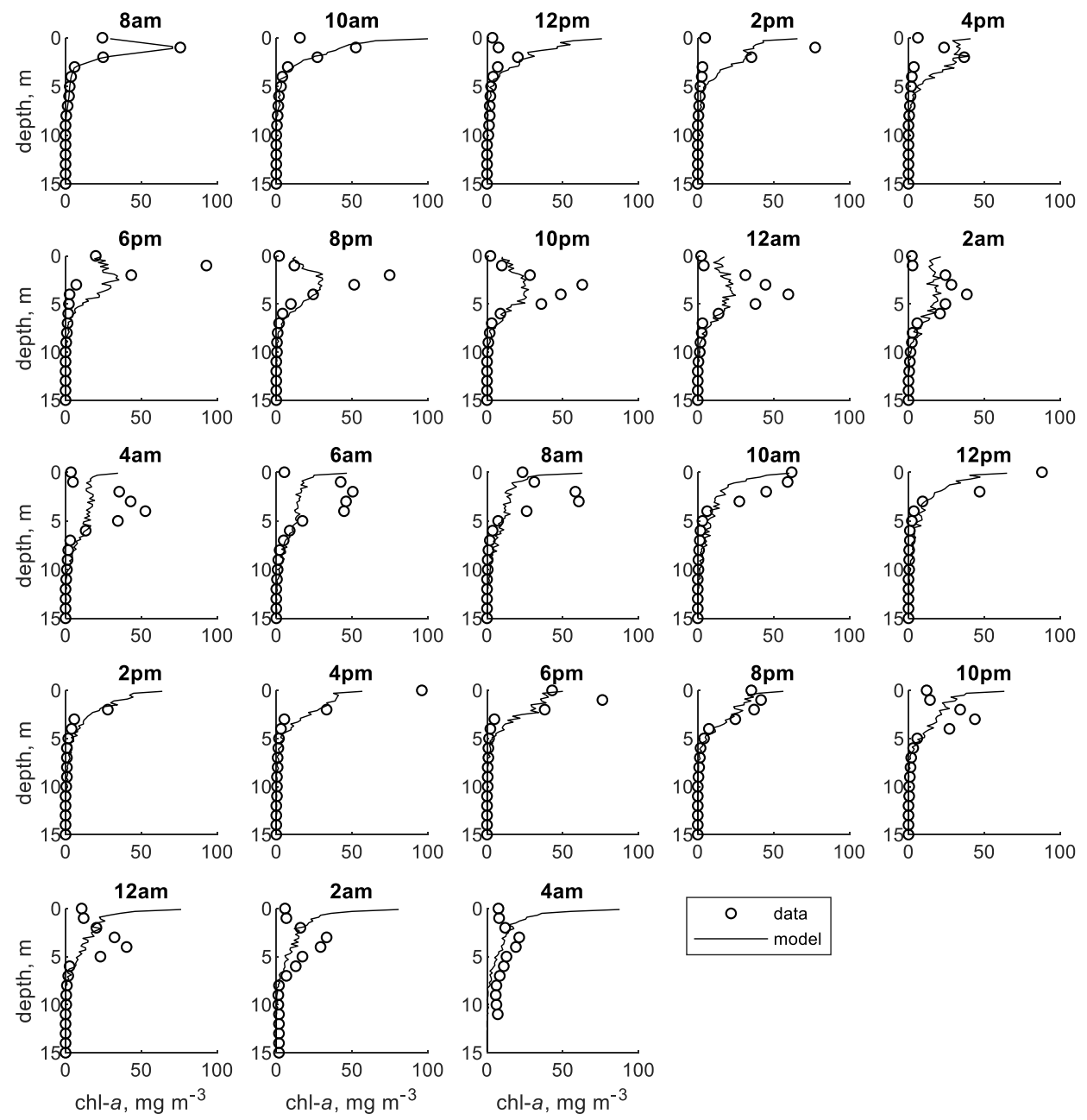

Figure A 23 Observed and predicted concentration profiles of chlorophyll a in Shennong Stream open water site using Visser et al. (1997) model (particle-tracking) 

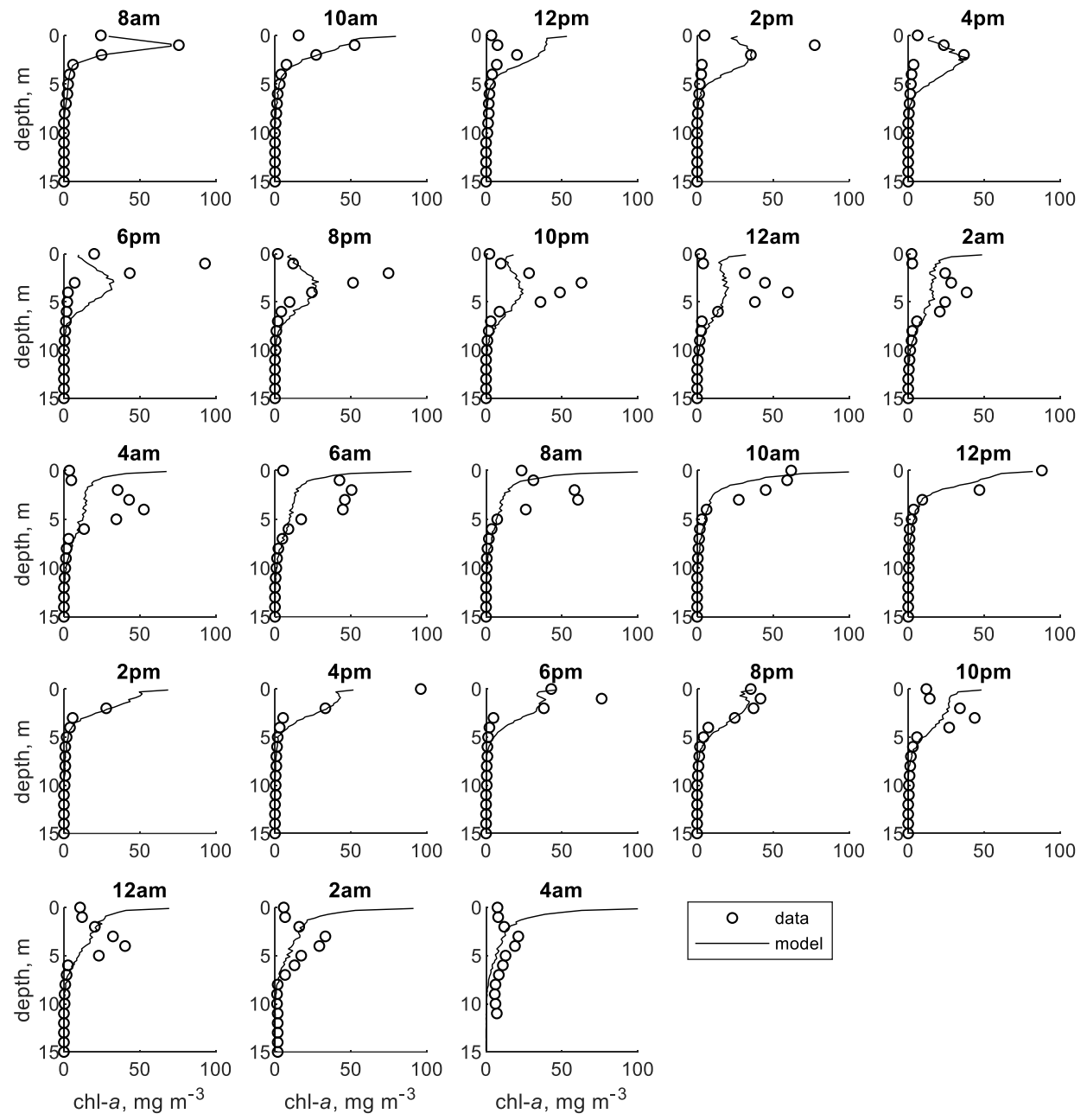

Figure A 24 Observed and predicted concentration profiles of chlorophyll a in Shennong Stream open water site using light function model (particle-tracking) 


\section{Appendix B: Contour Plots}
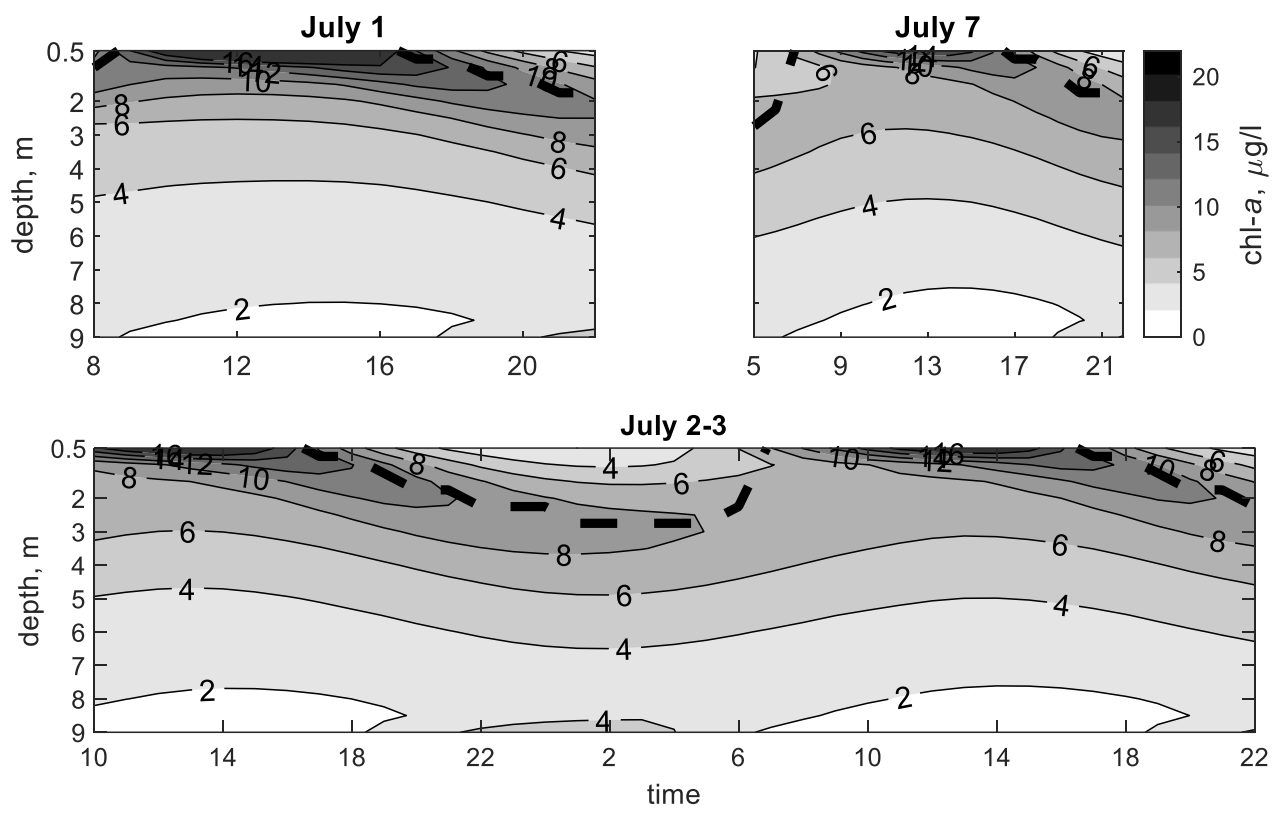

Figure B 1 Time series of chlorophyll a concentration contours and depth of maximum chlorophyll a concentration (dashed line) in Xiangxi Bay using continuum framework with time varying velocity model and $D_{z}=10^{-5} \mathrm{~m}^{2} \mathrm{~s}^{-1}$ 

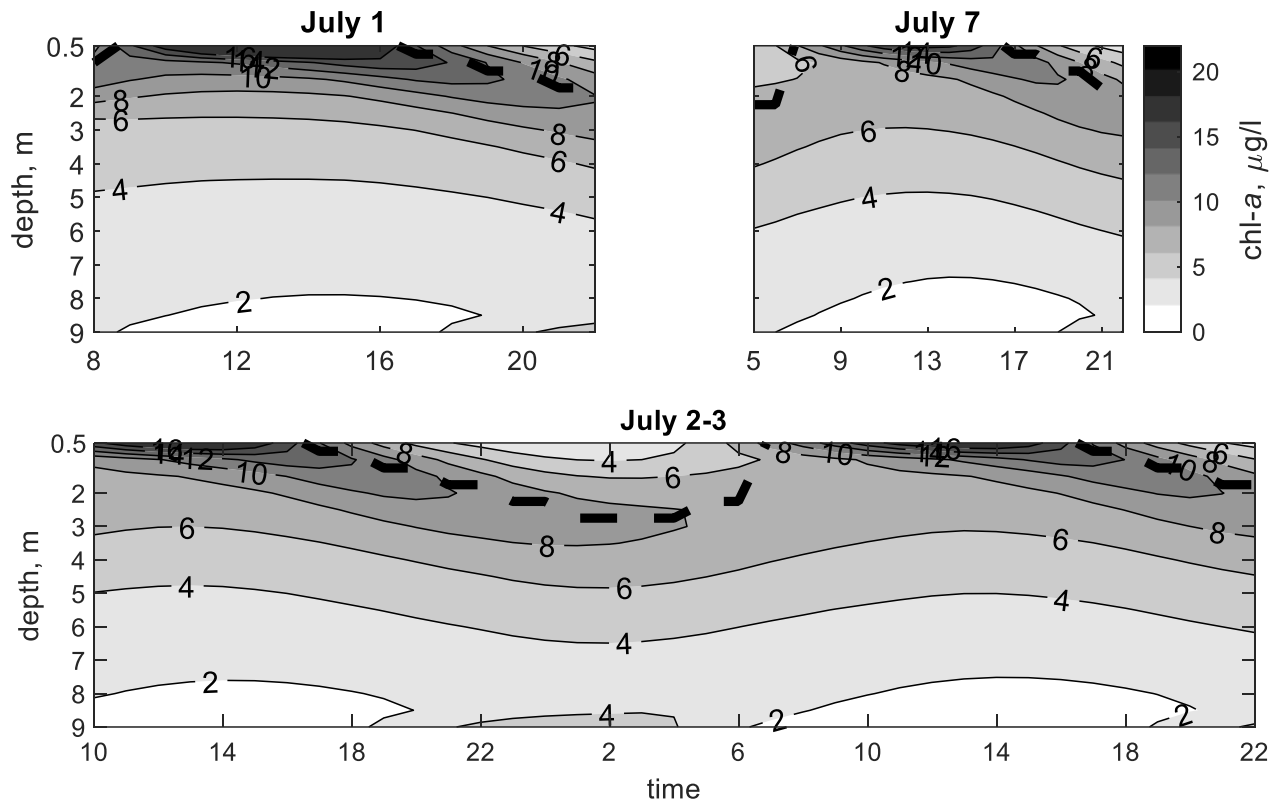

Figure B 2 Time series of chlorophyll a concentration contours and depth of maximum chlorophyll a concentration (dashed line) in Xiangxi Bay using continuum framework with Belov \& Giles (1997) model and $D_{z}=10^{-5} \mathrm{~m}^{2} \mathrm{~s}^{-1}$
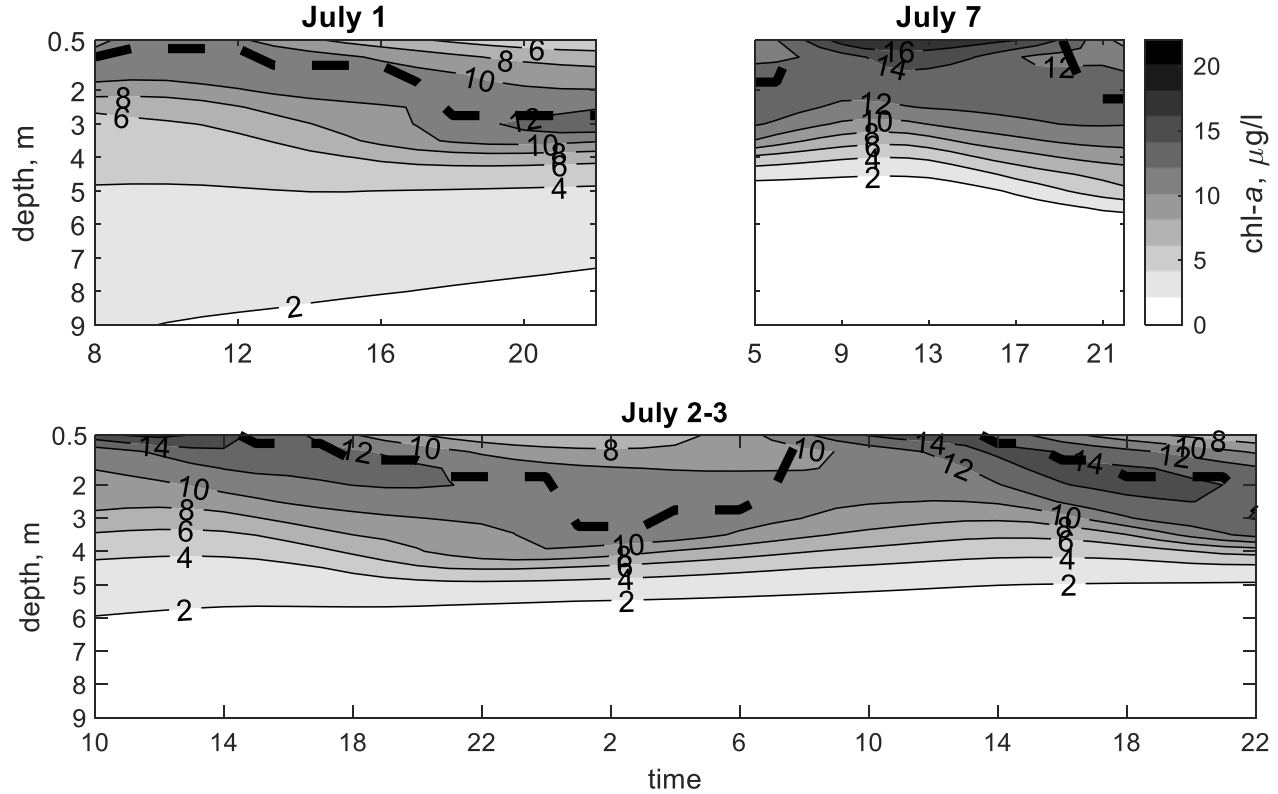

Figure B 3 Time series of chlorophyll a concentration contours and depth of maximum chlorophyll a concentration (dashed line) in Xiangxi Bay using continuum framework with growth kinetics model and $D_{z}=10^{-5} \mathrm{~m}^{2} \mathrm{~s}^{-1}$ 

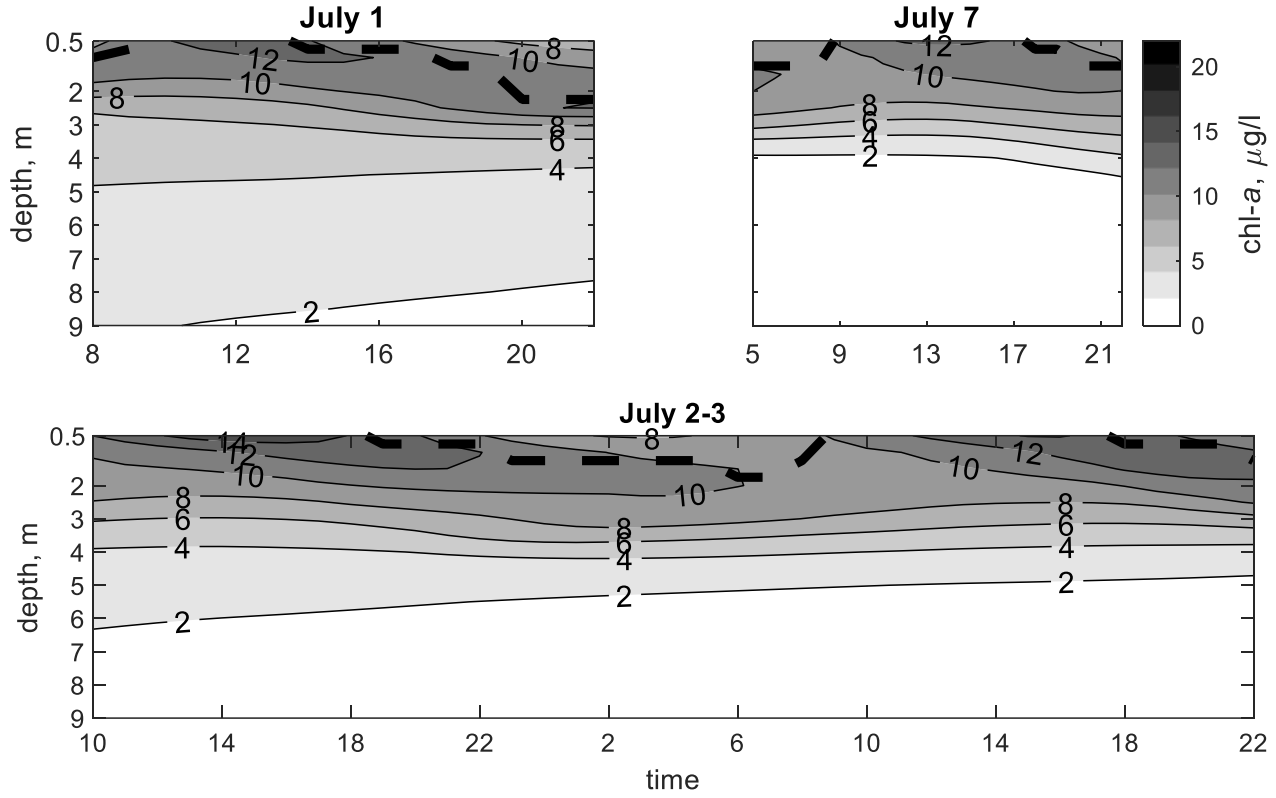

Figure B 4 Time series of chlorophyll a concentration contours and depth of maximum chlorophyll a concentration (dashed line) in Xiangxi Bay using continuum framework with growth kinetics (time decay) model and $D_{z}=10^{-5} \mathrm{~m}^{2} \mathrm{~s}^{-1}$
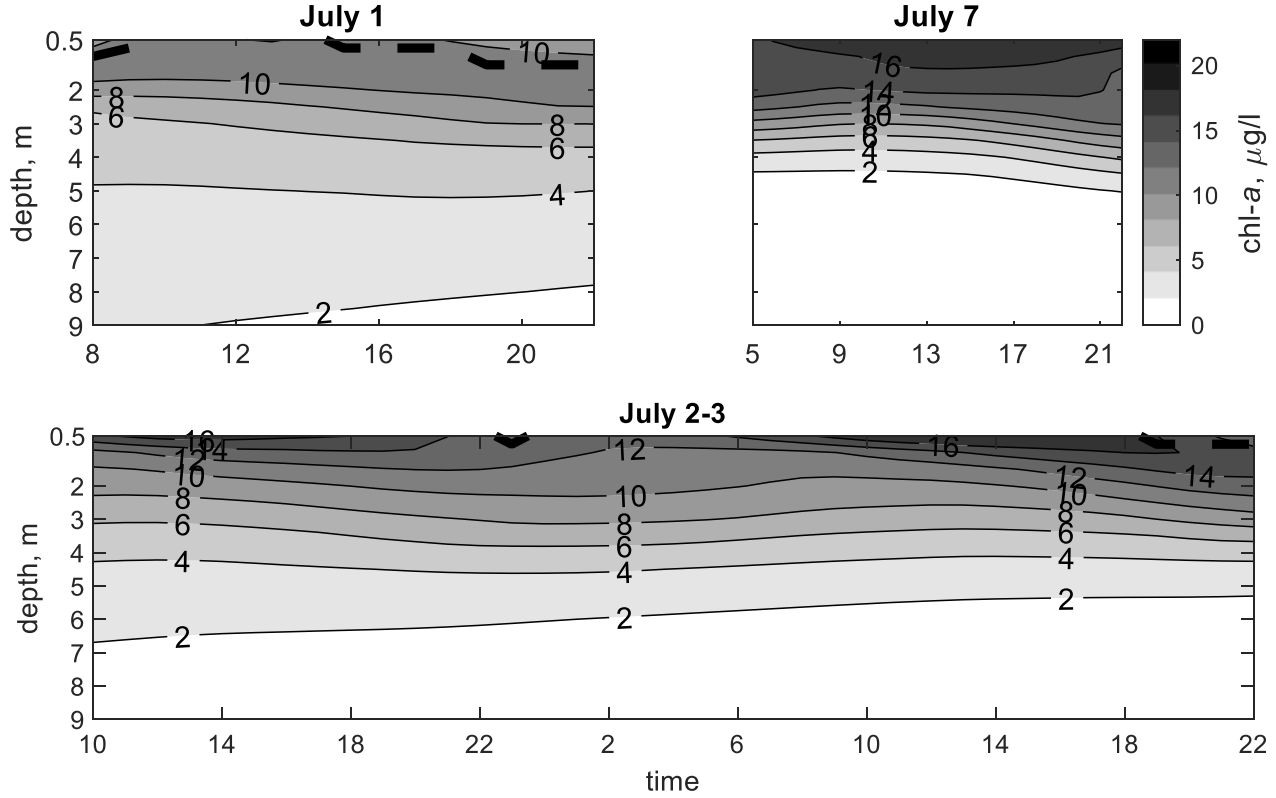

Figure B 5 Time series of chlorophyll a concentration contours and depth of maximum chlorophyll a concentration (dashed line) in Xiangxi Bay using continuum framework with Visser et al. (1997) model and $D_{z}=10^{-5} \mathrm{~m}^{2} \mathrm{~s}^{-1}$ 

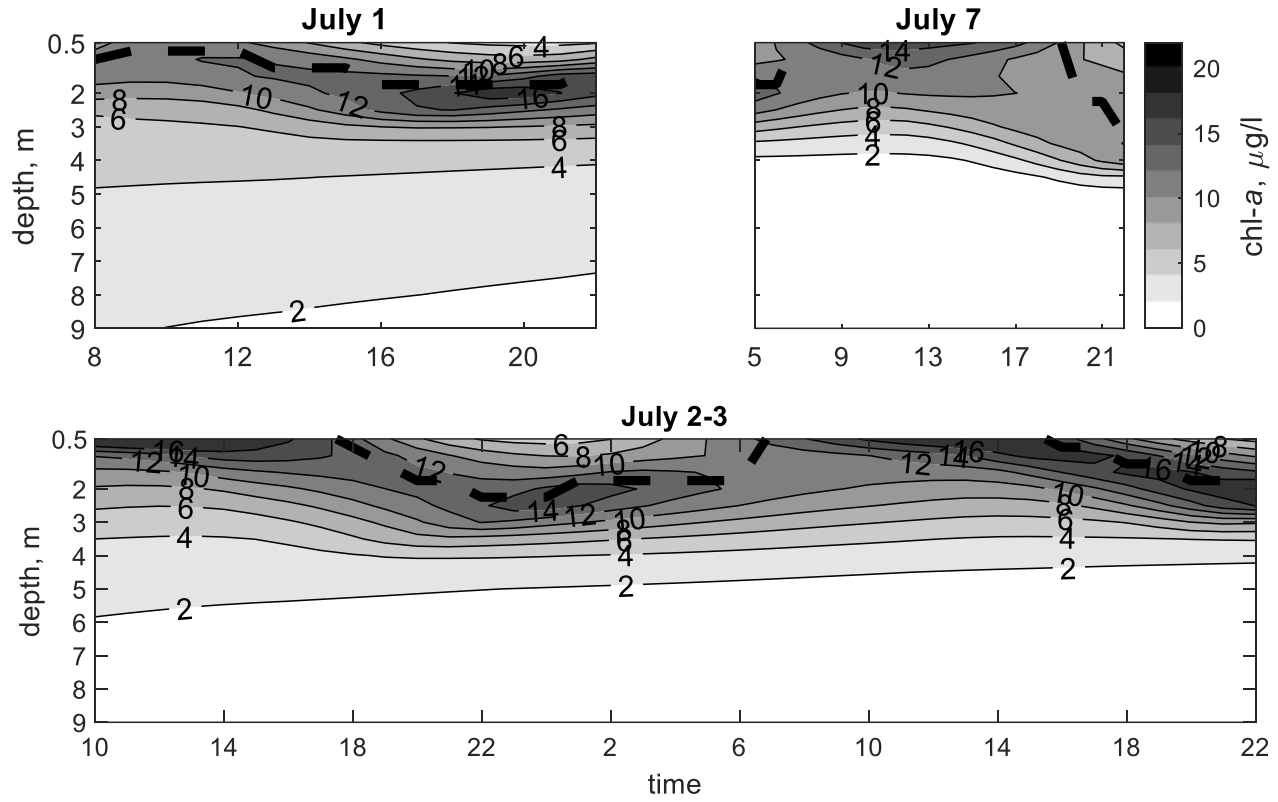

Figure B 6 Time series of chlorophyll a concentration contours and depth of maximum chlorophyll a concentration (dashed line) in Xiangxi Bay using continuum framework with light function model and $D_{z}=10^{-5} \mathrm{~m}^{2} \mathrm{~s}^{-1}$
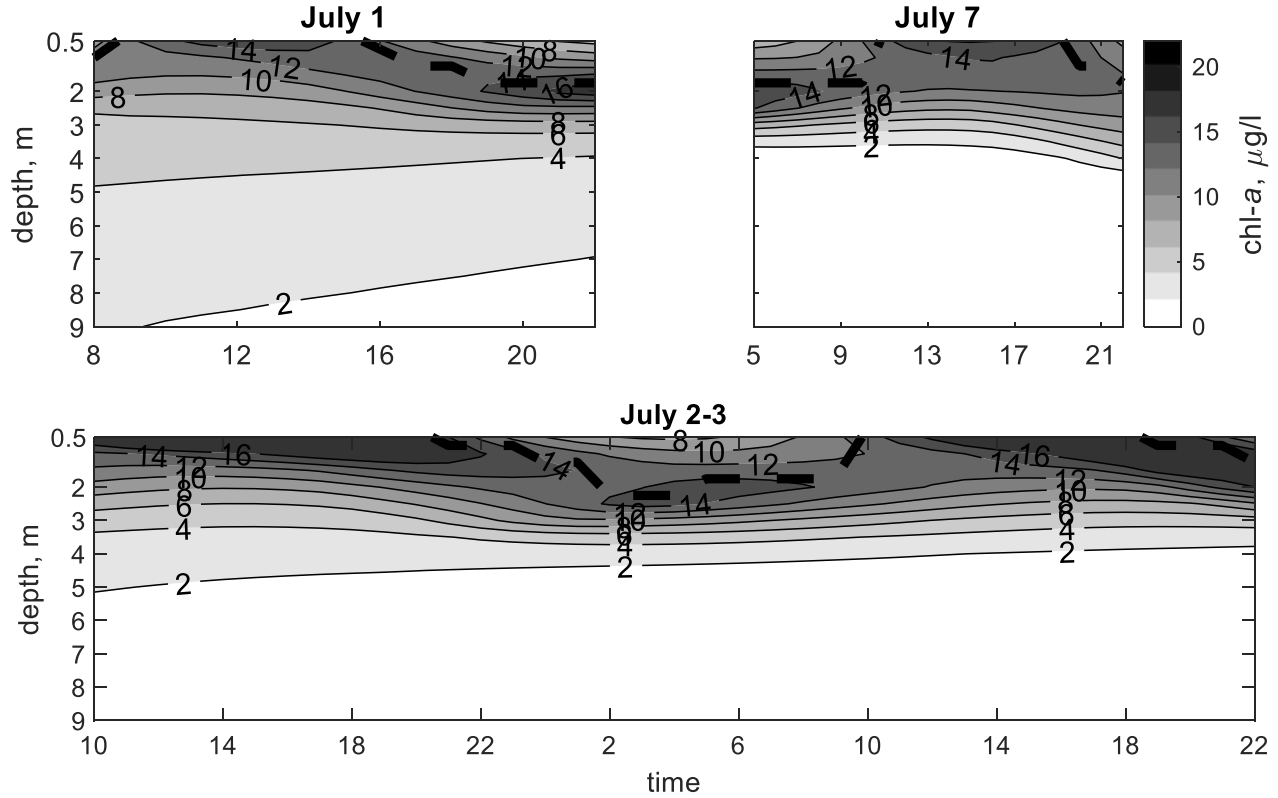

Figure B 7 Time series of chlorophyll a concentration contours and depth of maximum chlorophyll a concentration (dashed line) in Xiangxi Bay using continuum framework with light function (time decay) model and $D_{z}=10^{-5} \mathrm{~m}^{2} \mathrm{~s}^{-1}$ 

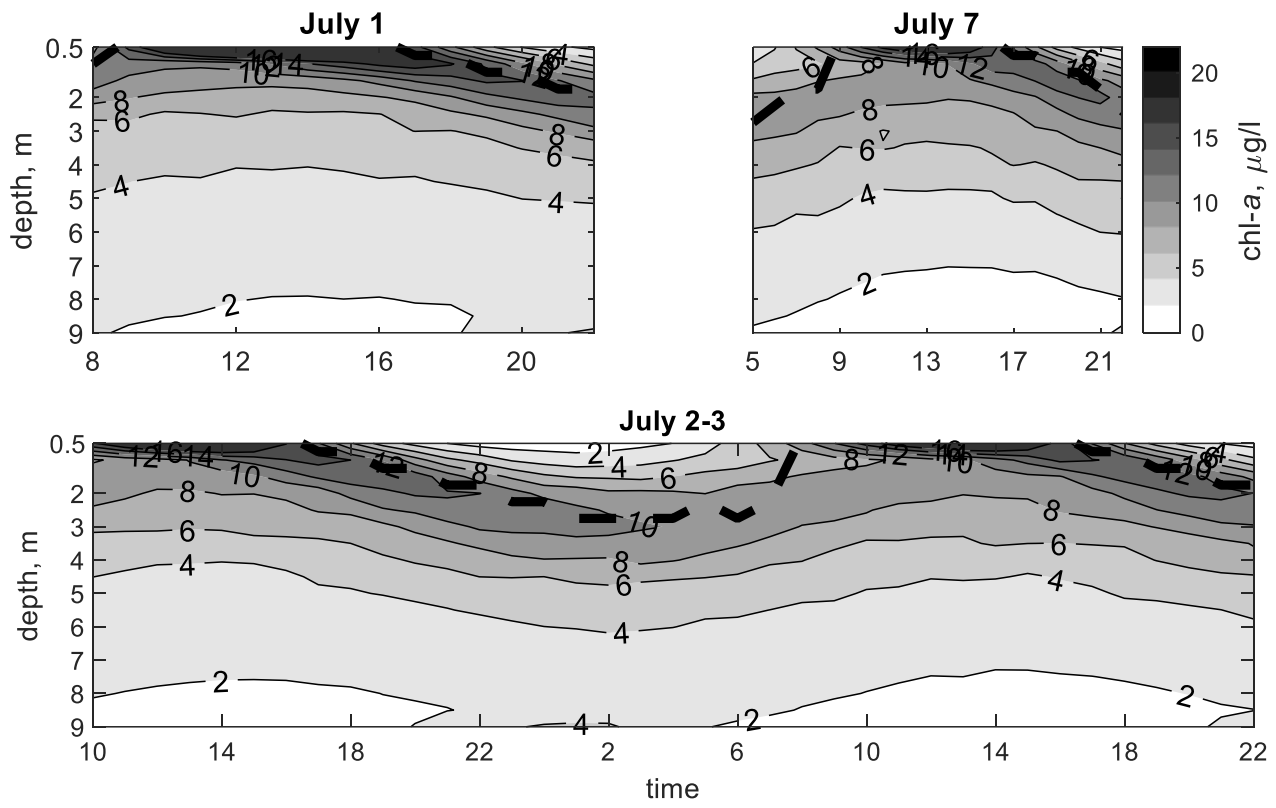

Figure B 8 Time series of chlorophyll a concentration contours and depth of maximum chlorophyll a concentration (dashed line) in Xiangxi Bay using particle-tracking framework with time-varying velocity model and $D_{z}=10^{-5} \mathrm{~m}^{2} \mathrm{~s}^{-1}$
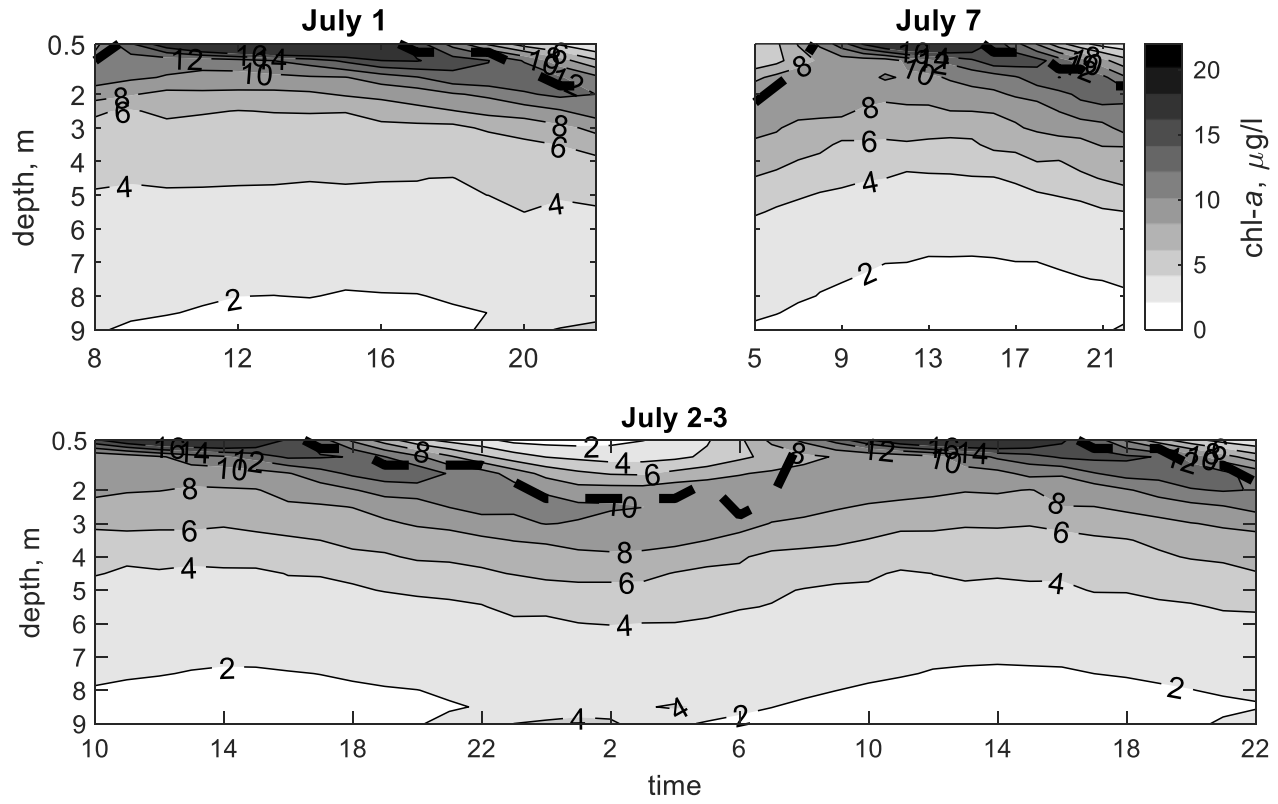

Figure B 9 Time series of chlorophyll a concentration contours and depth of maximum chlorophyll a concentration (dashed line) in Xiangxi Bay using particle-tracking framework with Belov \& Giles (1997) model and $D_{z}=10^{-5} \mathrm{~m}^{2} \mathrm{~s}^{-1}$ 

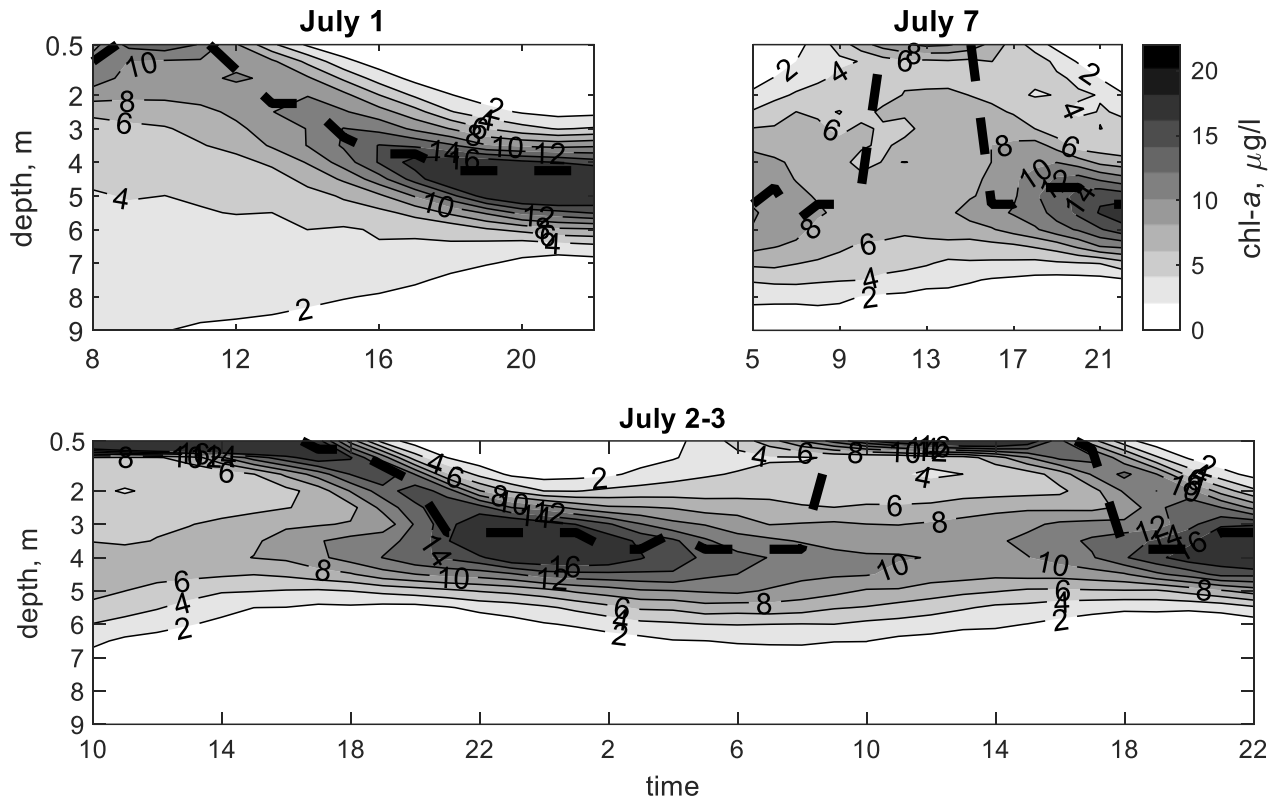

Figure B 10 Time series of chlorophyll a concentration contours and depth of maximum chlorophyll a concentration (dashed line) in Xiangxi Bay using particle-tracking framework with growth kinetics model and $D_{z}=10^{-5} \mathrm{~m}^{2} \mathrm{~s}^{-1}$
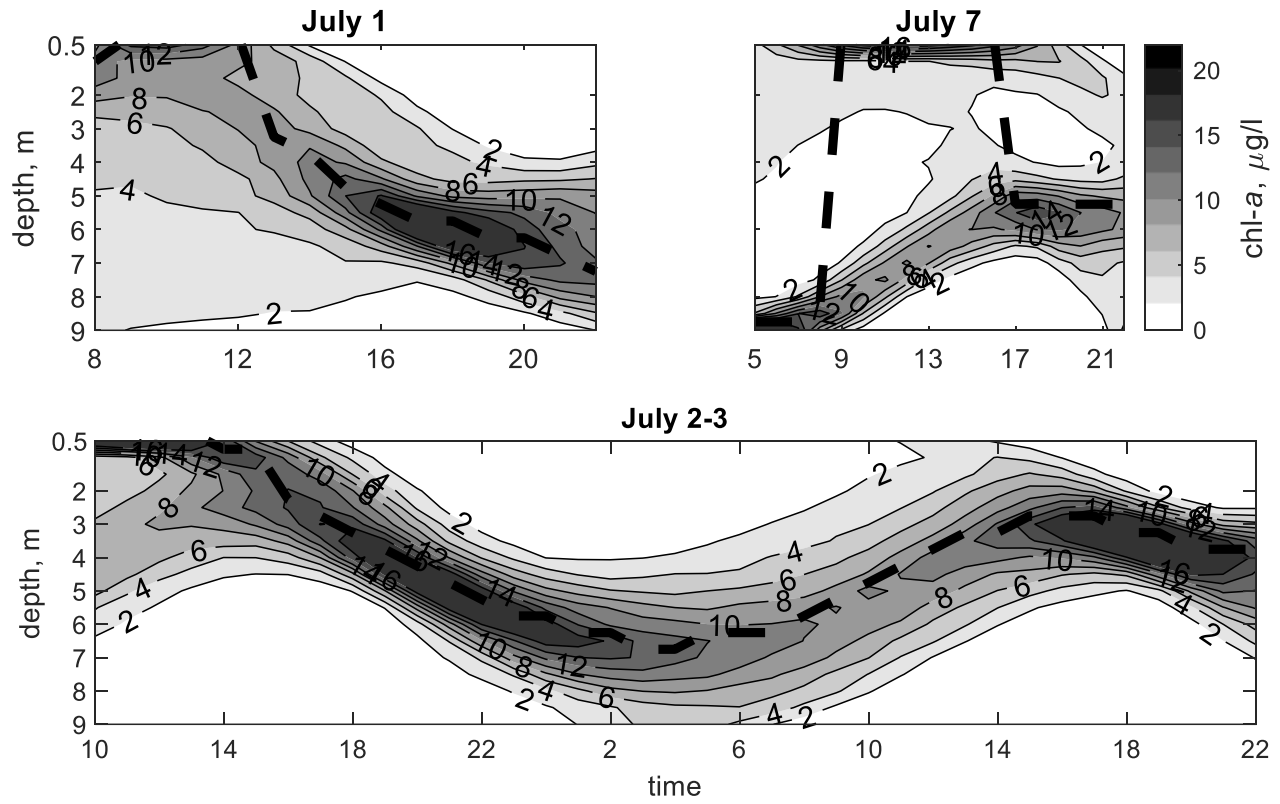

Figure B 11 Time series of chlorophyll a concentration contours and depth of maximum chlorophyll a concentration (dashed line) in Xiangxi Bay using particle-tracking framework with Visser et al. (1997) model and $D_{z}=10^{-5} \mathrm{~m}^{2} \mathrm{~s}^{-1}$ 

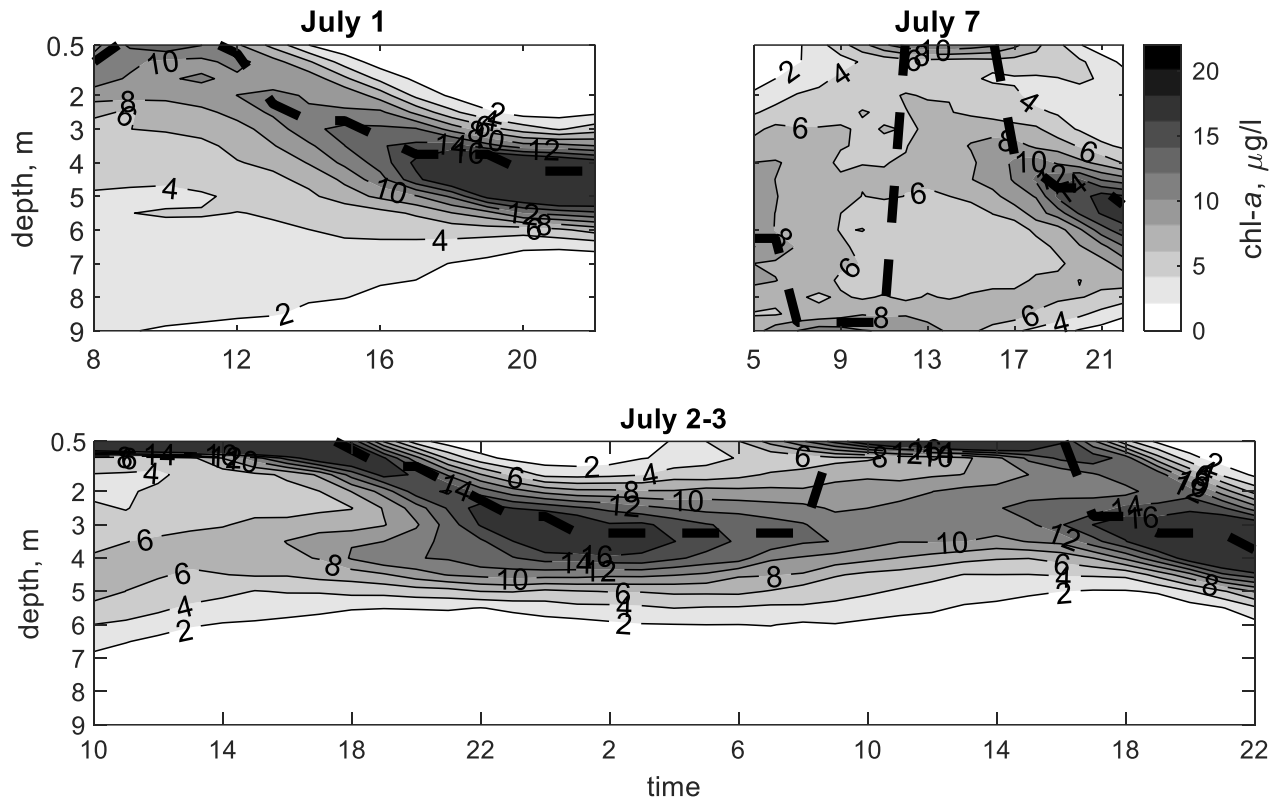

Figure B 12 Time series of chlorophyll a concentration contours and depth of maximum chlorophyll a concentration (dashed line) in Xiangxi Bay using particle-tracking framework with light function model and $D_{z}=10^{-5} \mathrm{~m}^{2} \mathrm{~s}^{-1}$
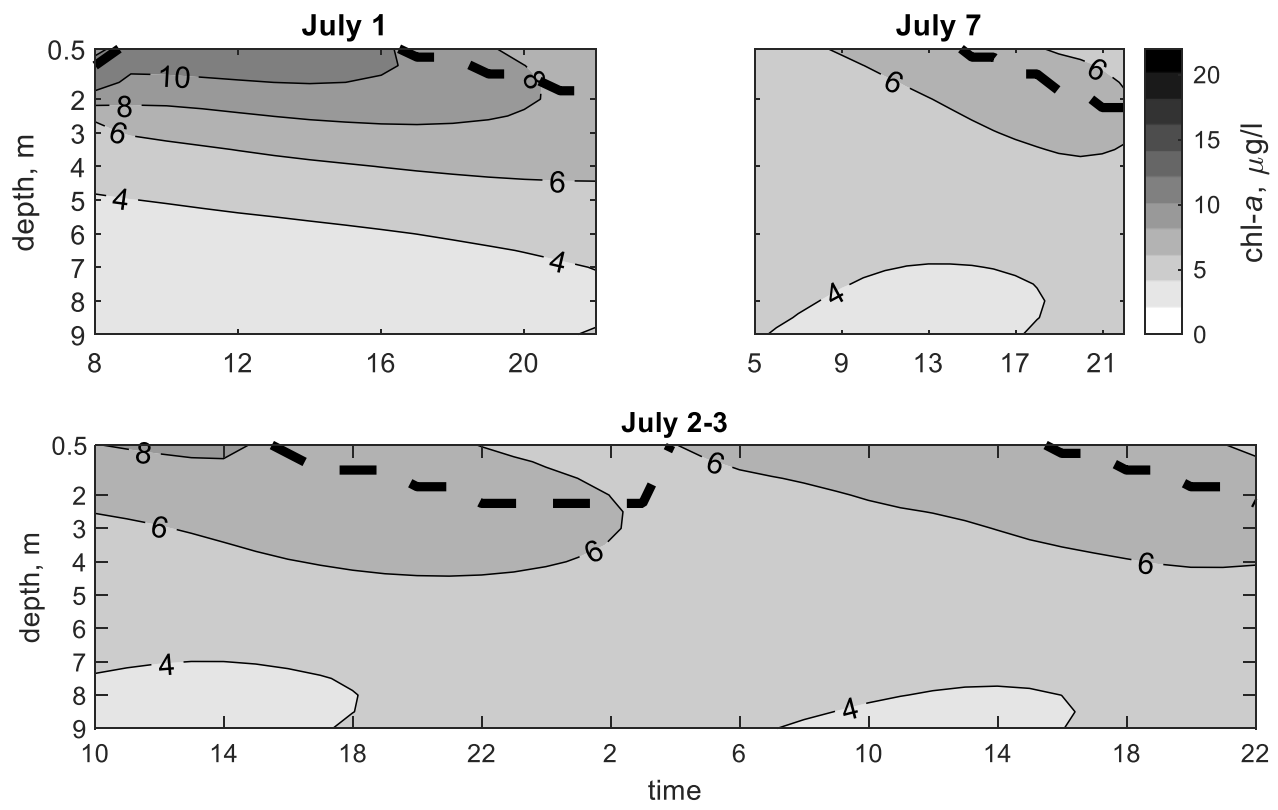

Figure B 13 Time series of chlorophyll a concentration contours and depth of maximum chlorophyll a concentration (dashed line) in Xiangxi Bay using continuum framework with time varying velocity model and $D_{z}=10^{-4} \mathrm{~m}^{2} \mathrm{~s}^{-1}$ 

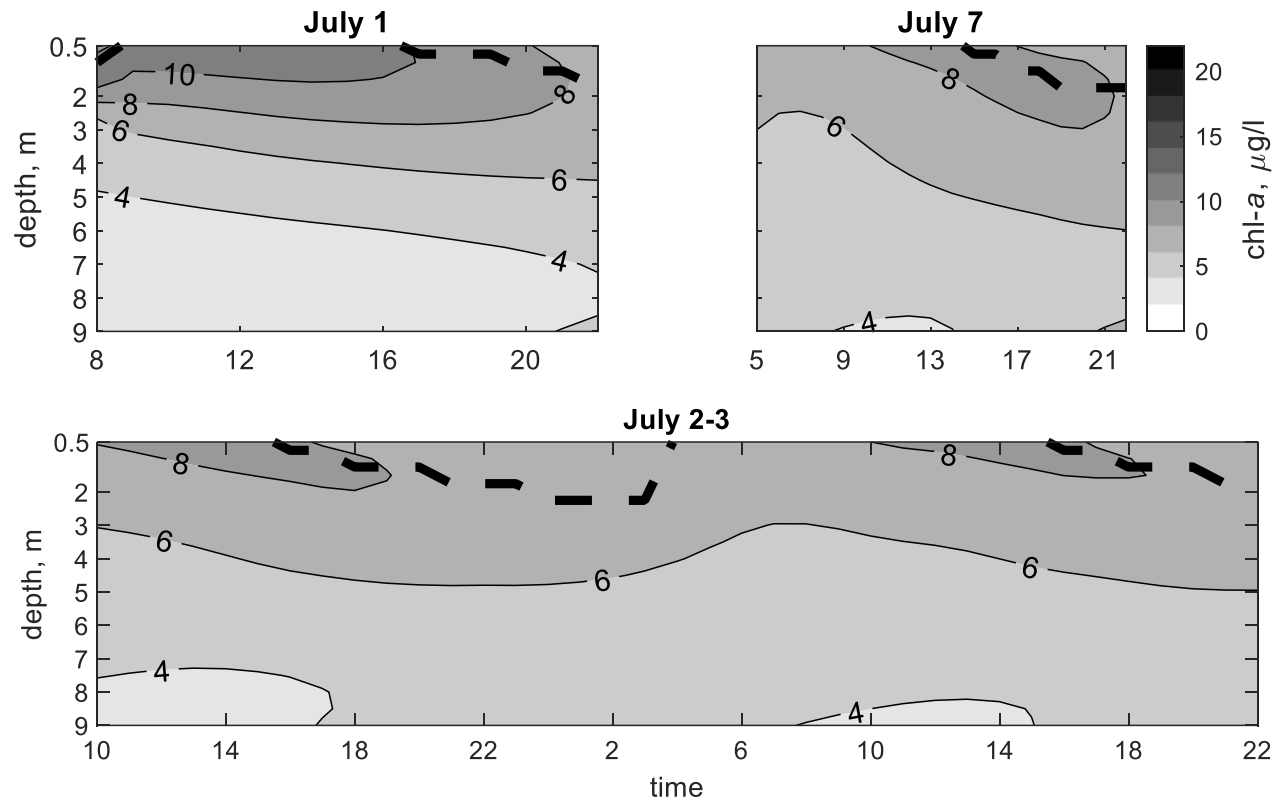

Figure B 14 Time series of chlorophyll a concentration contours and depth of maximum chlorophyll a concentration (dashed line) in Xiangxi Bay using continuum framework with Belov \& Giles (1997) model and $D_{z}=10^{-4} \mathrm{~m}^{2} \mathrm{~s}^{-1}$
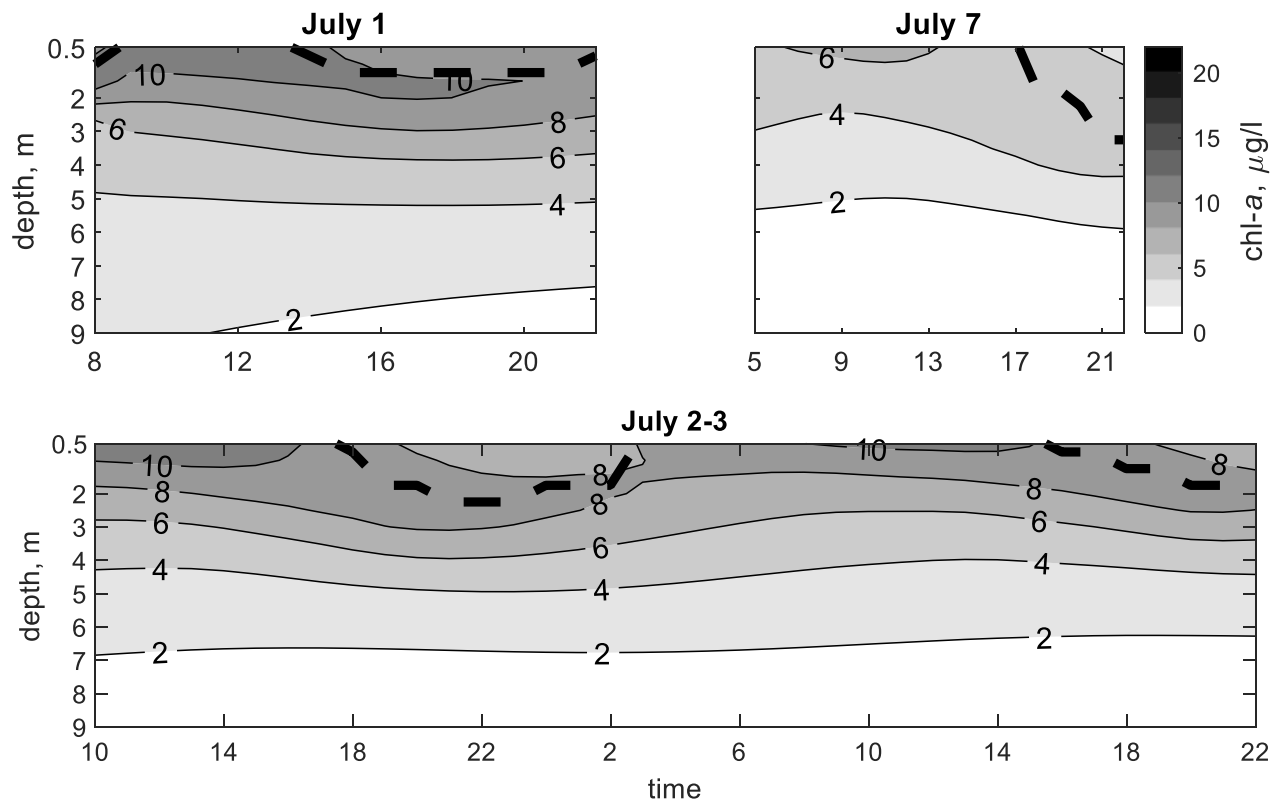

Figure B 15 Time series of chlorophyll a concentration contours and depth of maximum chlorophyll a concentration (dashed line) in Xiangxi Bay using continuum framework with growth kinetics model and $D_{z}=10^{-4} \mathrm{~m}^{2} \mathrm{~s}^{-1}$ 

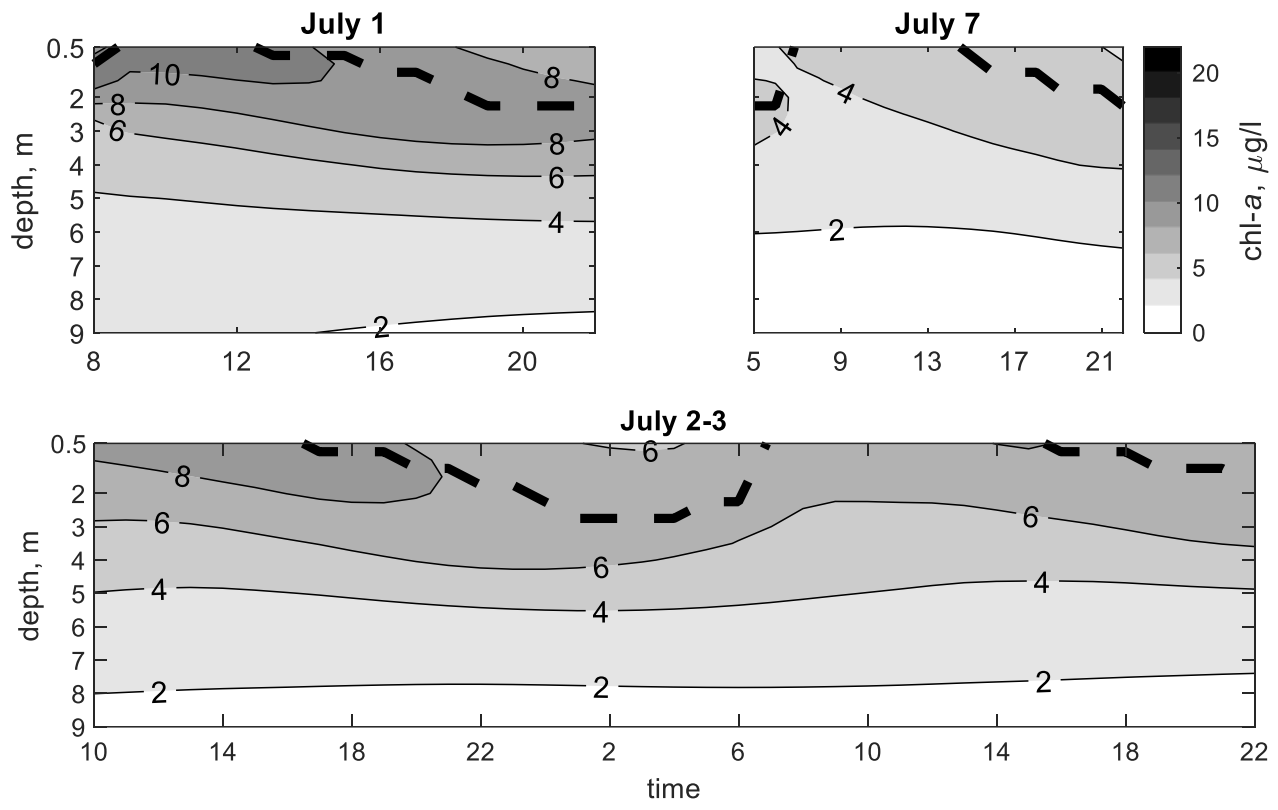

Figure B 16 Time series of chlorophyll a concentration contours and depth of maximum chlorophyll a concentration (dashed line) in Xiangxi Bay using continuum framework with growth kinetics (time decay) model and $D_{z}=10^{-4} \mathrm{~m}^{2} \mathrm{~s}^{-1}$
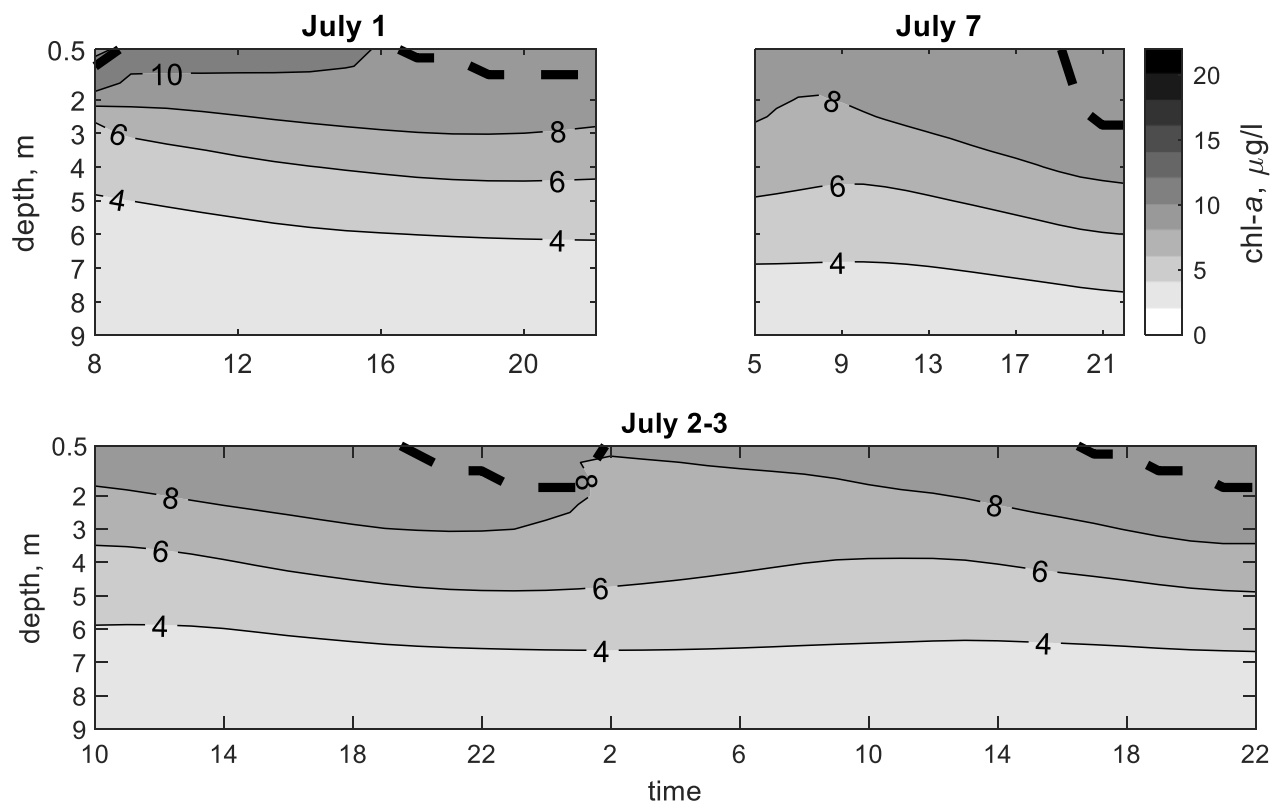

Figure B 17 Time series of chlorophyll a concentration contours and depth of maximum chlorophyll a concentration (dashed line) in Xiangxi Bay using continuum framework with Visser et al. (1997) model and $D_{z}=10^{-4} \mathrm{~m}^{2} \mathrm{~s}^{-1}$ 

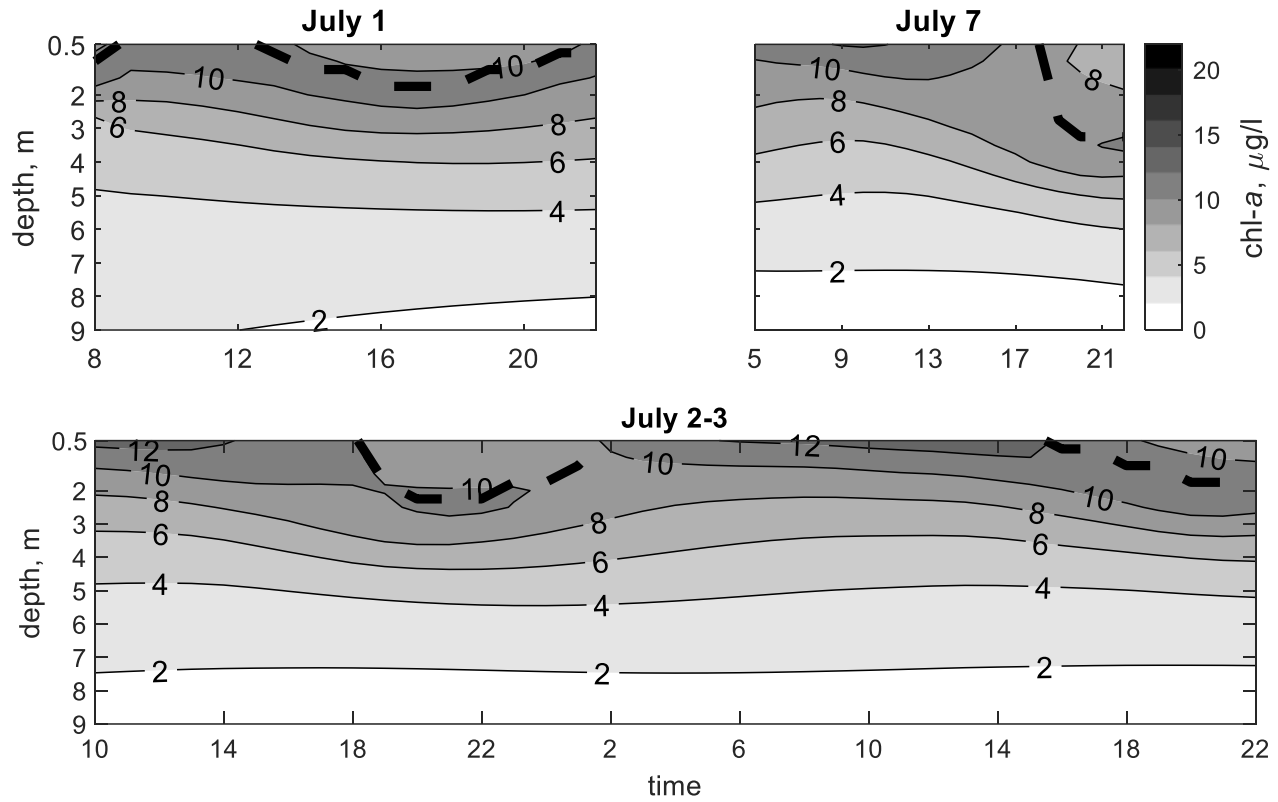

Figure B 18 Time series of chlorophyll a concentration contours and depth of maximum chlorophyll a concentration (dashed line) in Xiangxi Bay using continuum framework with light function model and $D_{z}=10^{-4} \mathrm{~m}^{2} \mathrm{~s}^{-1}$
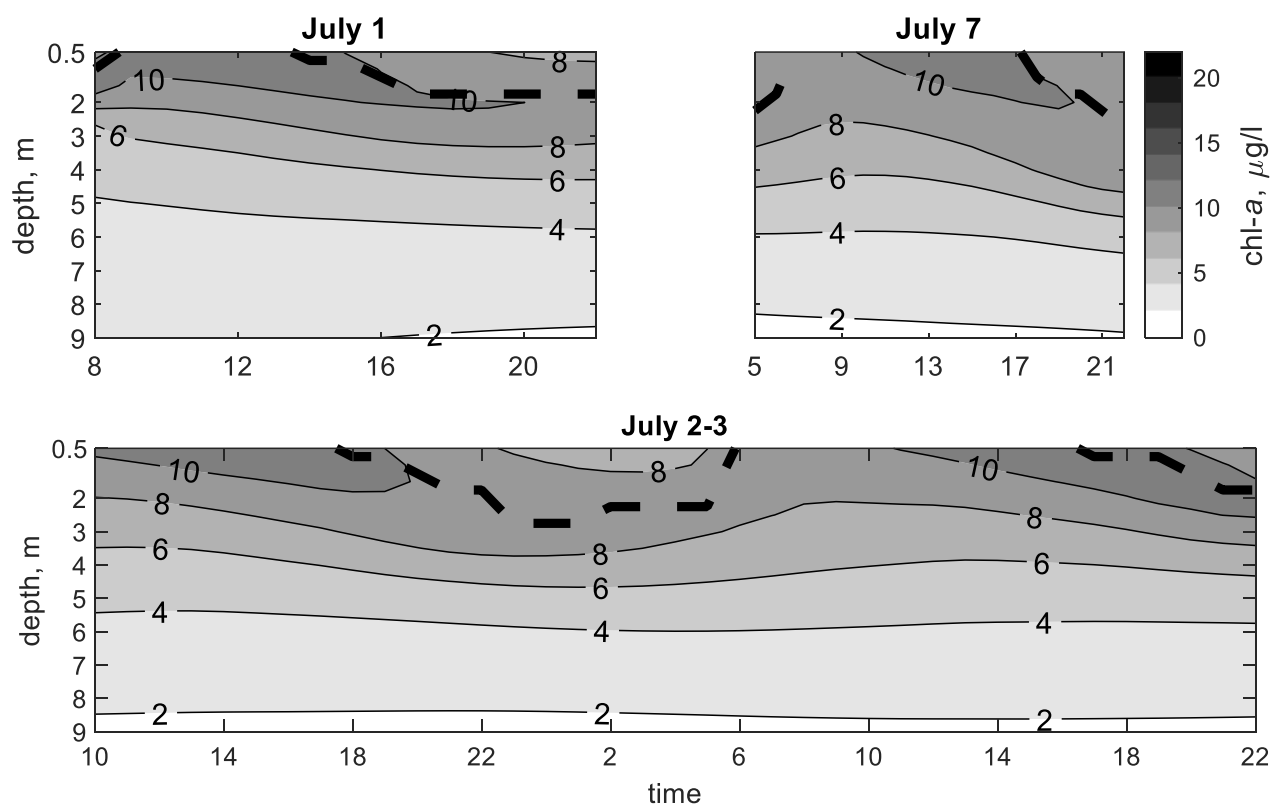

Figure B 19 Time series of chlorophyll a concentration contours and depth of maximum chlorophyll a concentration (dashed line) in Xiangxi Bay using continuum framework with light function (time decay) model and $D_{z}=10^{-4} \mathrm{~m}^{2} \mathrm{~s}^{-1}$ 

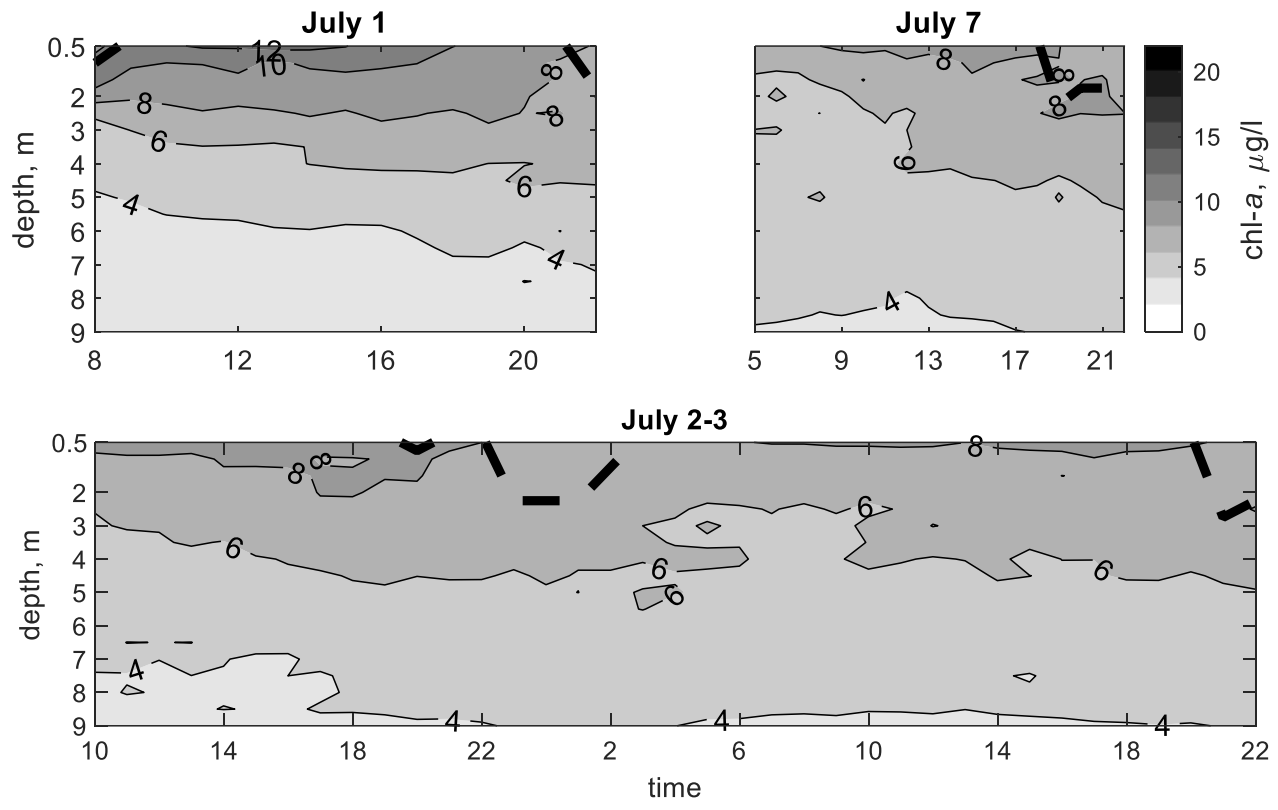

Figure B 20 Time series of chlorophyll a concentration contours and depth of maximum chlorophyll a concentration (dashed line) in Xiangxi Bay using particle-tracking framework with time-varying velocity model and $D_{z}=10^{-4} \mathrm{~m}^{2} \mathrm{~s}^{-1}$
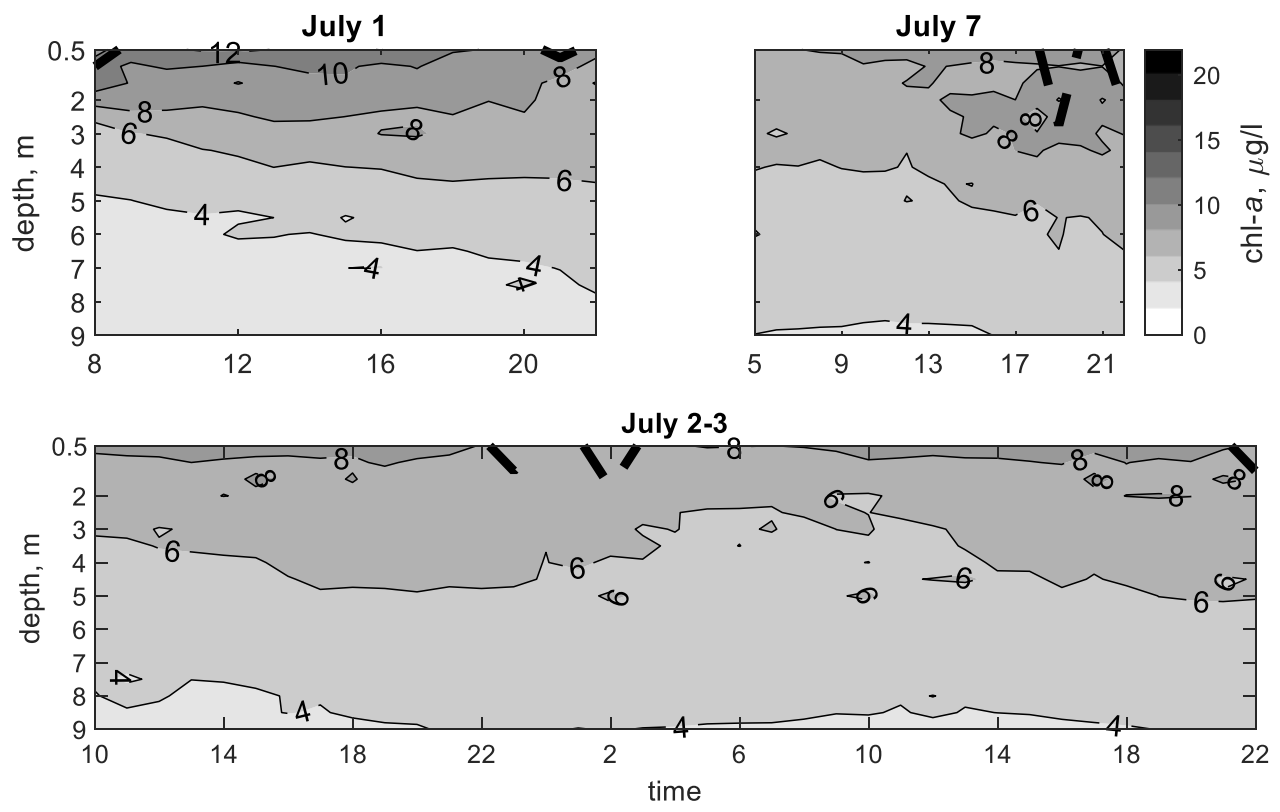

Figure B 21 Time series of chlorophyll a concentration contours and depth of maximum chlorophyll a concentration (dashed line) in Xiangxi Bay using particle-tracking framework with Belov \& Giles (1997) model and $D_{z}=10^{-4} \mathrm{~m}^{2} \mathrm{~s}^{-1}$ 

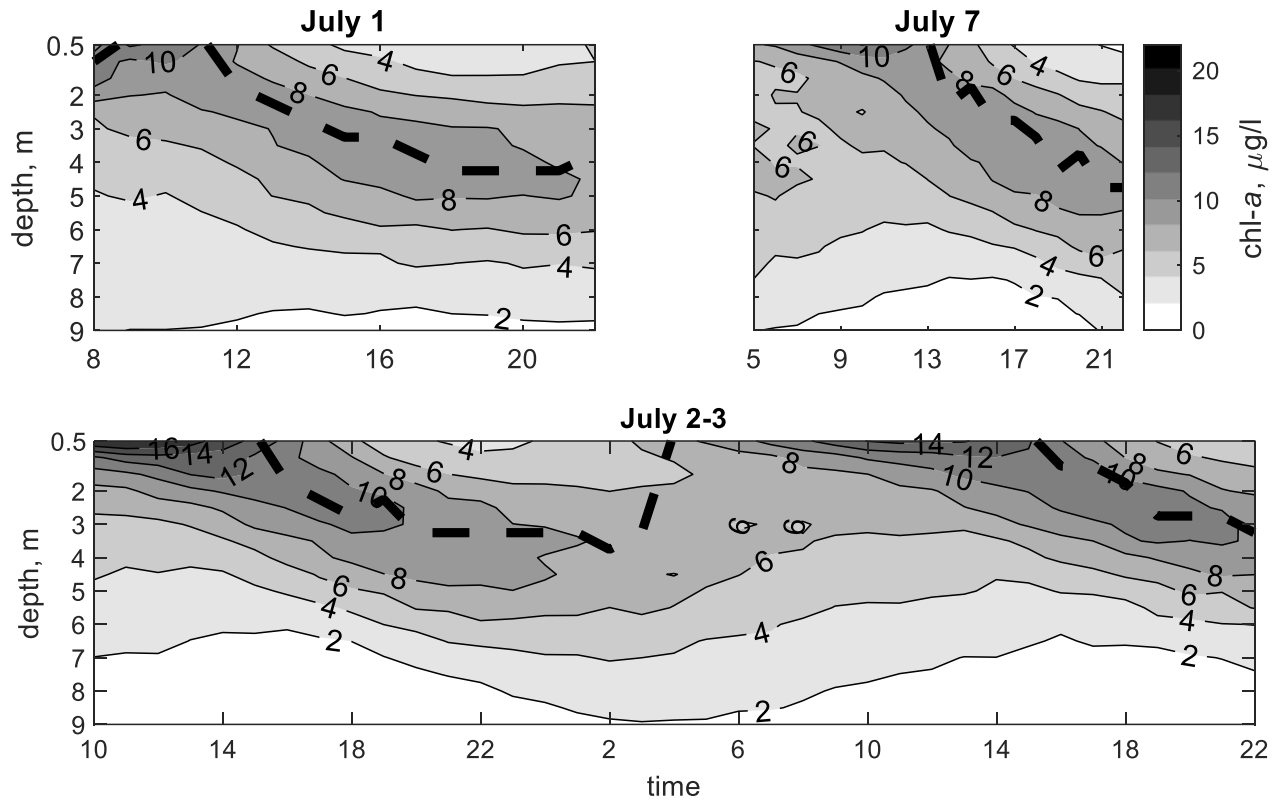

Figure B 22 Time series of chlorophyll a concentration contours and depth of maximum chlorophyll a concentration (dashed line) in Xiangxi Bay using particle-tracking framework with growth kinetics model and $D_{z}=10^{-4} \mathrm{~m}^{2} \mathrm{~s}^{-1}$
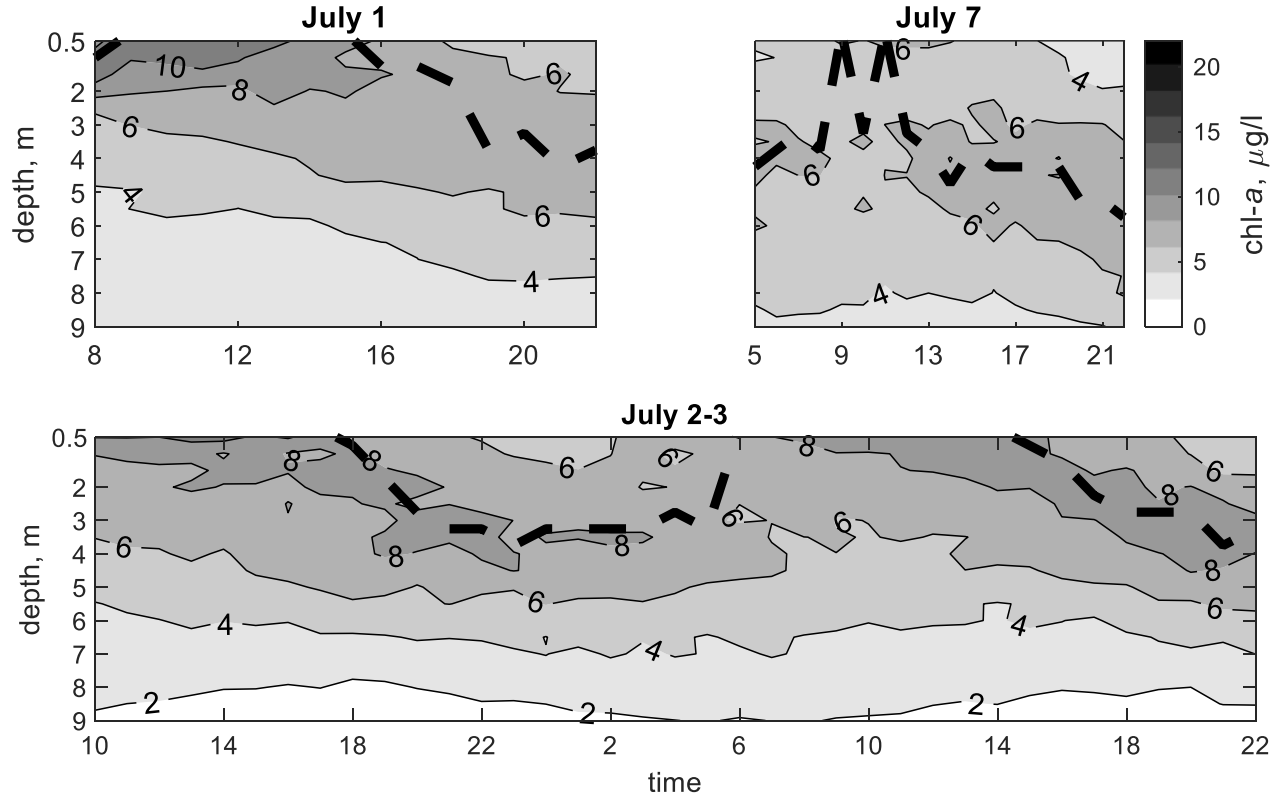

Figure B 23 Time series of chlorophyll a concentration contours and depth of maximum chlorophyll a concentration (dashed line) in Xiangxi Bay using particle-tracking framework with Visser et al. (1997) model and $D_{z}=10^{-4} \mathrm{~m}^{2} \mathrm{~s}^{-1}$ 

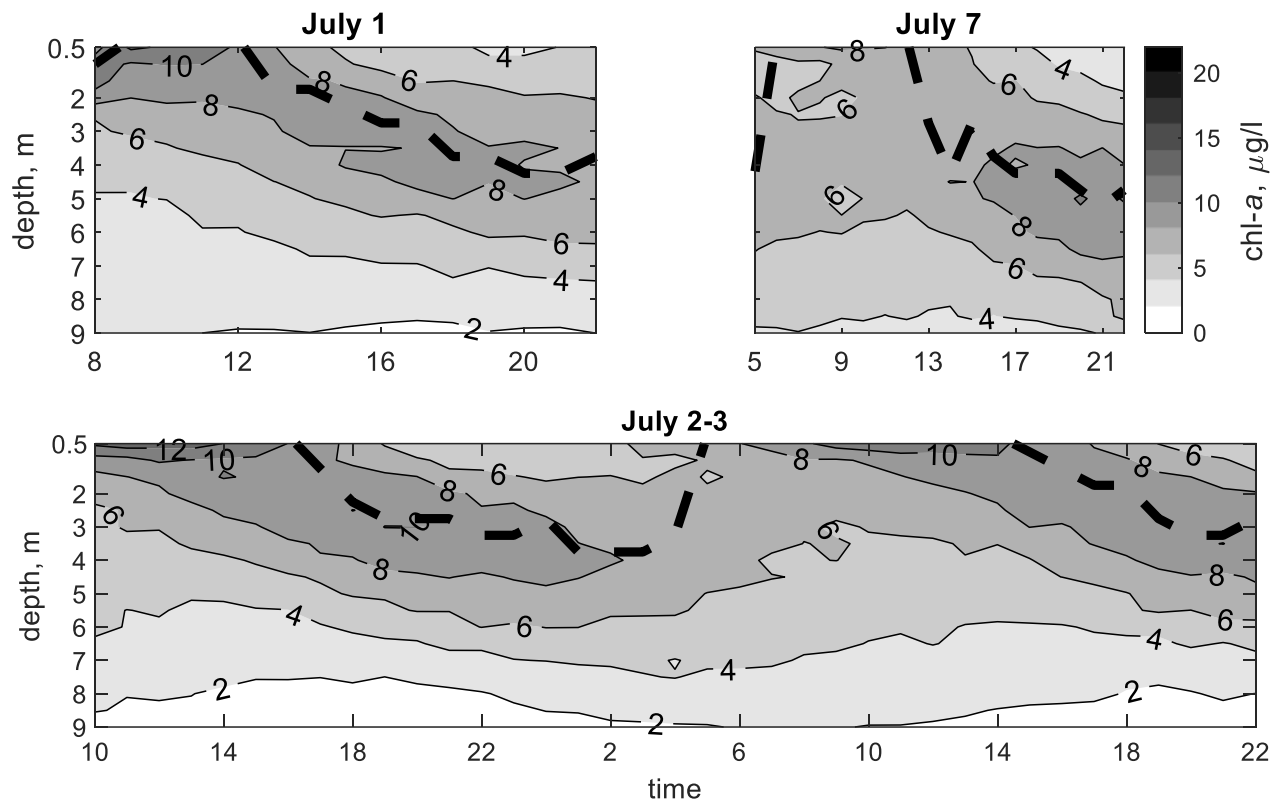

Figure B 24 Time series of chlorophyll a concentration contours and depth of maximum chlorophyll a concentration (dashed line) in Xiangxi Bay using particle-tracking framework with light function model and $D_{z}=10^{-4} \mathrm{~m}^{2} \mathrm{~s}^{-1}$ 


\section{Appendix C: CE-QUAL-W2 Code}

\section{Below is the fortran90 code of the CE-QUAL-W2 Water Quality Subroutine. New}

\section{code additions from this study are highlighted.}

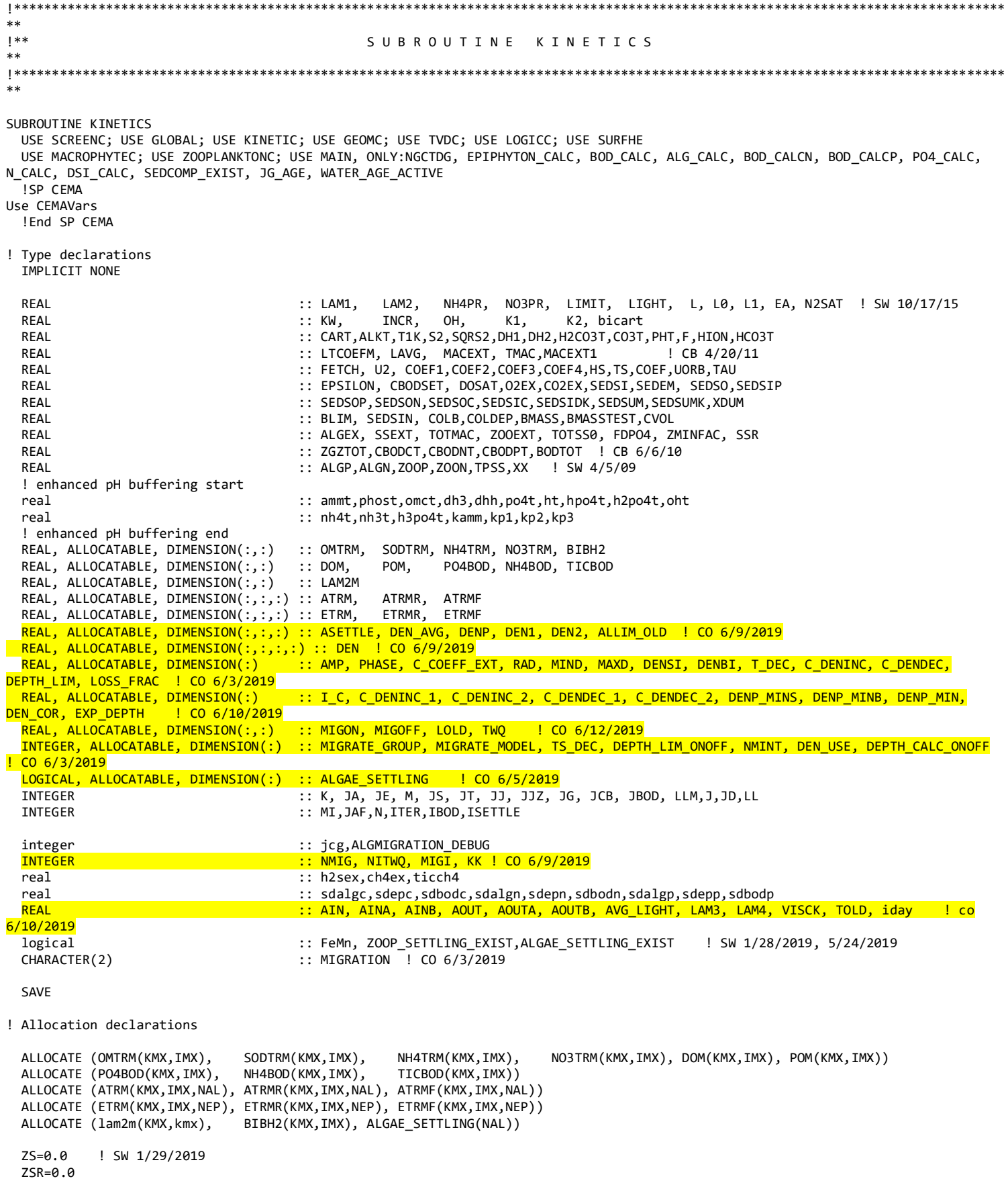




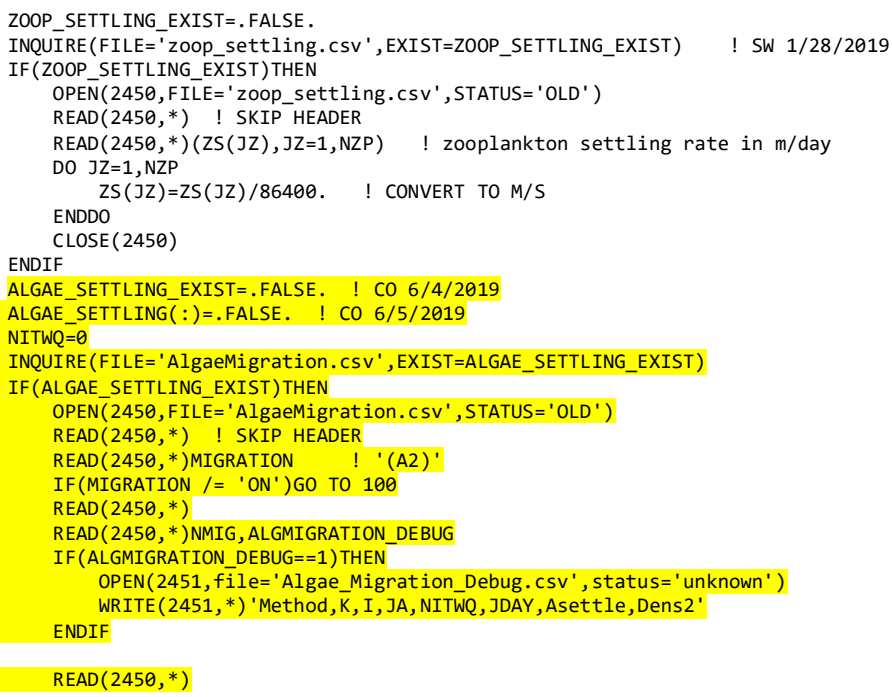

ALLOCATE(MIGRATE_GROUP(NMIG), MIGRATE_MODEL(NMIG), AMP(NMIG), PHASE(NMIG), C_COEFF_EXT(NMIG), RAD(NMIG), MIND(NMIG), MAXD(NMIG), DENSI(NMI G), DENBI (NMIG), T_DEC(NMIG), TS_DEC (NMIG), C_DENINC (NMIG), C_DENDEC (NMIG), \&

DEPTH_LIM_ONOFF(NMIG), DEPTH_LIM(NMIG), LOSS_FRAC(NMIG), I_C(NMIG), C_DENINC_1(NMIG), C_DENINC_2(NMIG), C_DENDEC_1(NMIG), C_DENDEC_2(NMIG ),DENP_MINS(NMIG), DENP_MINB(NMIG), DEN_COR(MIGI), \&

NMINT(NMIG), MIGON(NMIG, 12), MIGOFF (NMIG, 12), DEPTH_CALC_ONOFF(NMIG), EXP_DEPTH(NMIG))
DO I=1,NMIG

$\operatorname{READ}(2450, *) \operatorname{MIGRATE} G$ GROUP $(\mathrm{I})$

$\operatorname{READ}(2450, *)$

$\operatorname{READ}(2450, *) \operatorname{NMINT}(\mathrm{I})$

$\operatorname{READ}(2450, *)$

$\operatorname{READ}(2450, *) \operatorname{MIGON}(I, 1: \operatorname{NMINT}(\mathrm{I}))$

$\operatorname{READ}(2450, *)$

$\operatorname{READ}(2450, *) \operatorname{MIGOFF}(\mathrm{I}, 1$ :NMINT(I))

$\operatorname{READ}(2450, *)$

$\operatorname{READ}(2450, *)$ MIGRATE_MODEL (I)

$\operatorname{READ}(2450, *)$

$\operatorname{IF}\left(\operatorname{MIGRATE} \_\right.$MODEL $(I)==1 \quad$.OR. MIGRATE_MODEL $\left.(I)==2\right)$ THEN

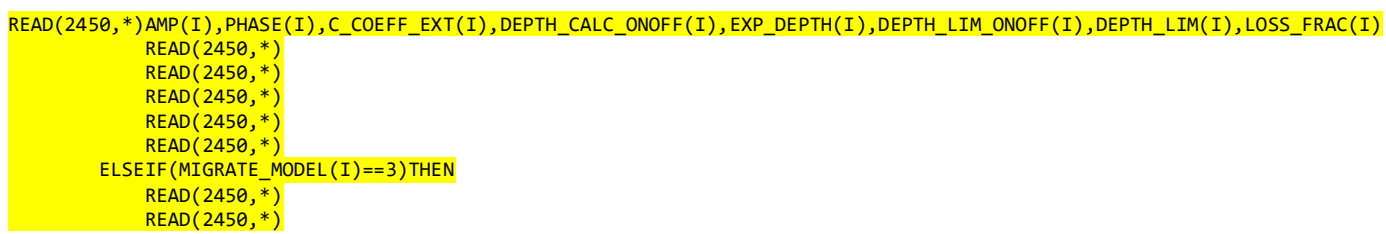

$\operatorname{READ}(2450, *) \operatorname{RAD}(\mathrm{I}), \operatorname{MIND}(\mathrm{I}), \operatorname{MAXD}(\mathrm{I}), \operatorname{DENSI}(\mathrm{I}), \operatorname{DENBI}(\mathrm{I}), \mathrm{T} D \mathrm{DEC}(\mathrm{I}), \mathrm{TS} D E C(\mathrm{I}), \mathrm{C}$ DENINC(I), C_DENDEC(I), DEPTH_LIM_ONOFF(I), DEPTH_LIM(I), LO SS_FRAC (I)

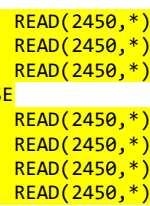

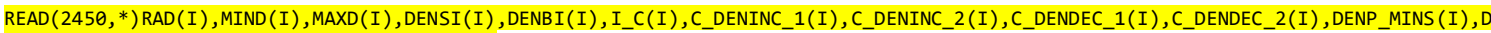
ENP_MINB(I), DEN_COR(I), DEPTH_LIM_ONOFF (I), \& DEPTH_LIM(I), LOSS_FRAC (I)

ALLOCATE(DEN_AVG (KMX, IMX, NMIG), DEN(KMX, IMX, MAXVAL (TS_DEC), NMIG), DEN1(KMX, IMX, NMIG), DEN2 (KMX, IMX, NMIG), DENP (KMX, IMX, NMIG), DENP_MIN( KMX), ASETTLE (KMX, IMX, NAL), ALLIM OLD (KMX, IMX, NMIG),

RETURN 


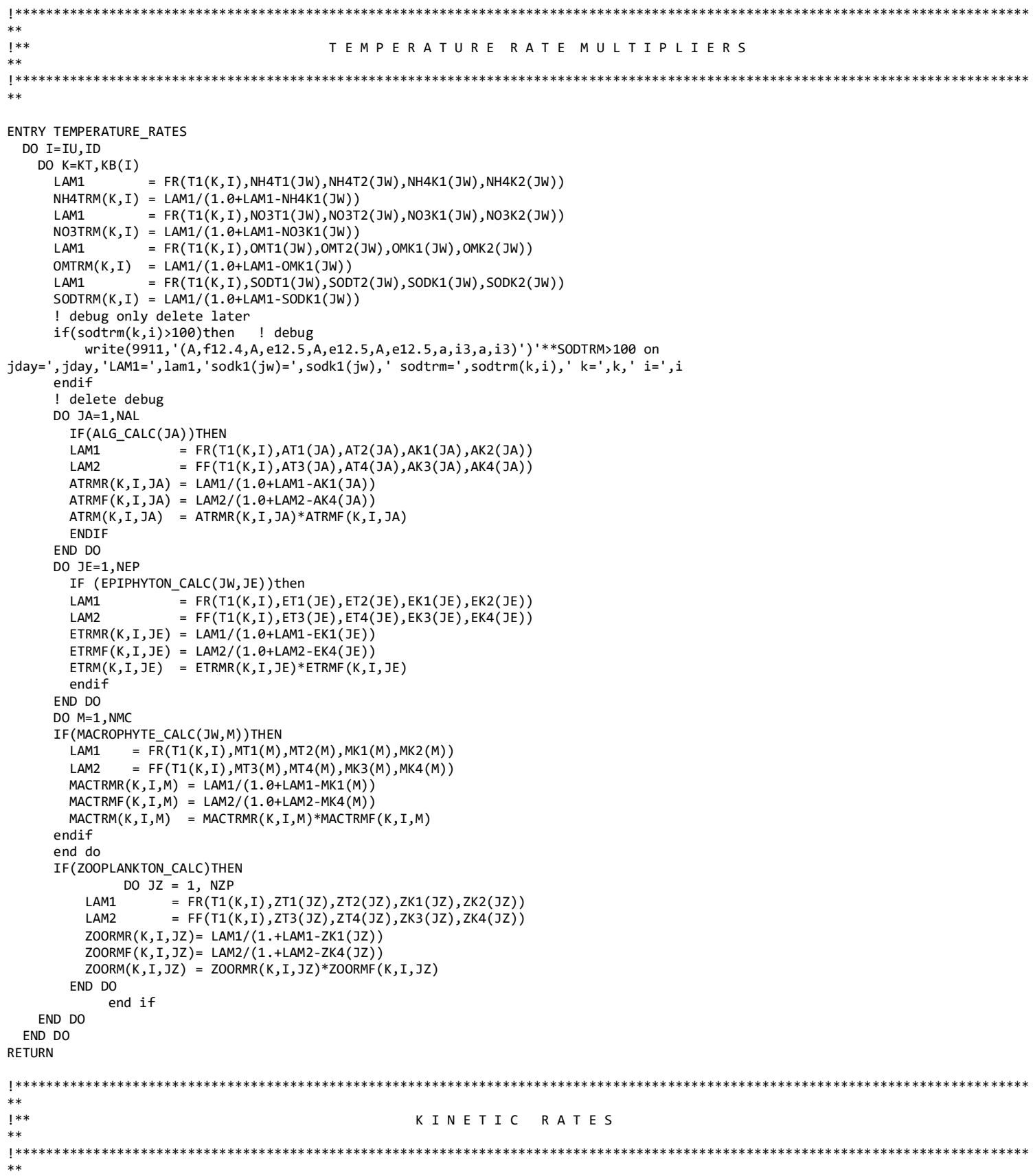

ENTRY KINETIC_RATES

! Decay rates

!!\$OMP PARALLEL DO

DO I=IU, ID

$\mathrm{DO} \quad \mathrm{K}=\mathrm{KT}, \mathrm{KB}(\mathrm{I})$

$\mathrm{D01}(\mathrm{K}, \mathrm{I}) \quad=02(\mathrm{~K}, \mathrm{I}) /(\mathrm{O} 2(\mathrm{~K}, \mathrm{I})+\mathrm{KDO})$

$\operatorname{DO2}(\mathrm{K}, \mathrm{I}) \quad=1.0-\operatorname{D01}(\mathrm{K}, \mathrm{I}) \quad \mathrm{O} 2(\mathrm{~K}, \mathrm{I}) /(02(\mathrm{~K}, \mathrm{I})+\mathrm{KDO})$

$\operatorname{DO3}(K, I)=(1.0+\operatorname{SIGN}(1.0,02(K, I)-1 . E-10)) * 0.5$

! debug only delete later

if $(\operatorname{do} 2(k, i)>100$. ) then ! debug

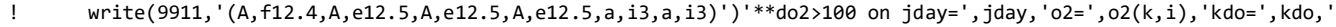

do $2={ }^{\prime}, \operatorname{do} 2(k, i),{ }^{\prime} k={ }^{\prime}, k,{ }^{\prime} i={ }^{\prime}, i$

! endif

! ! delete debug 


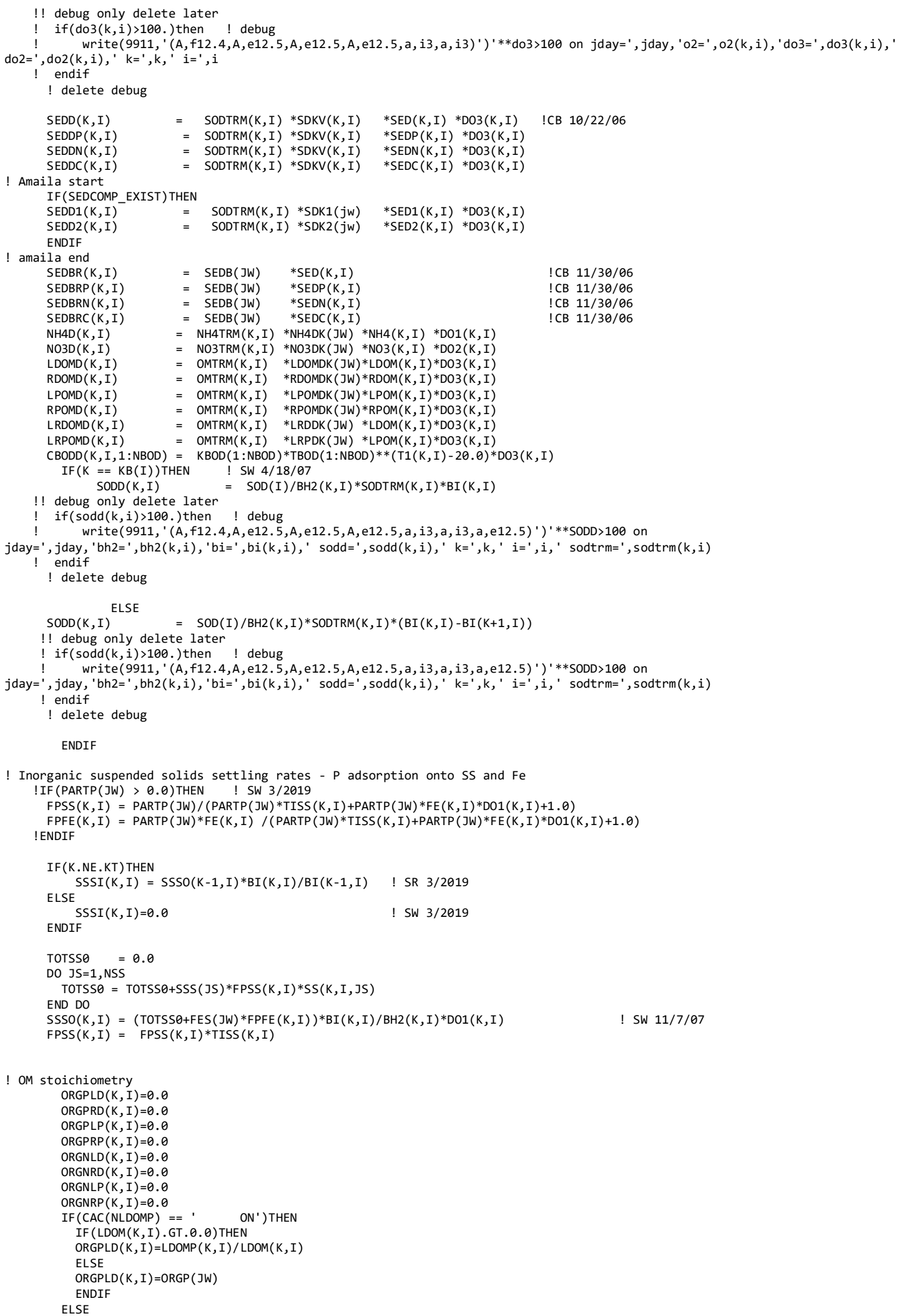




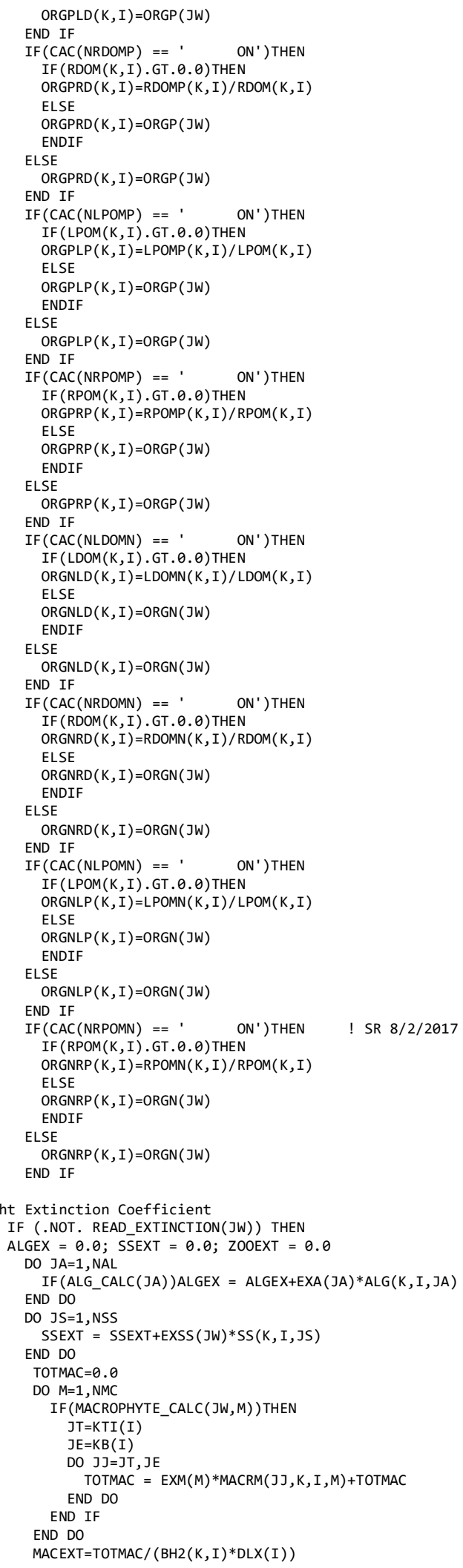




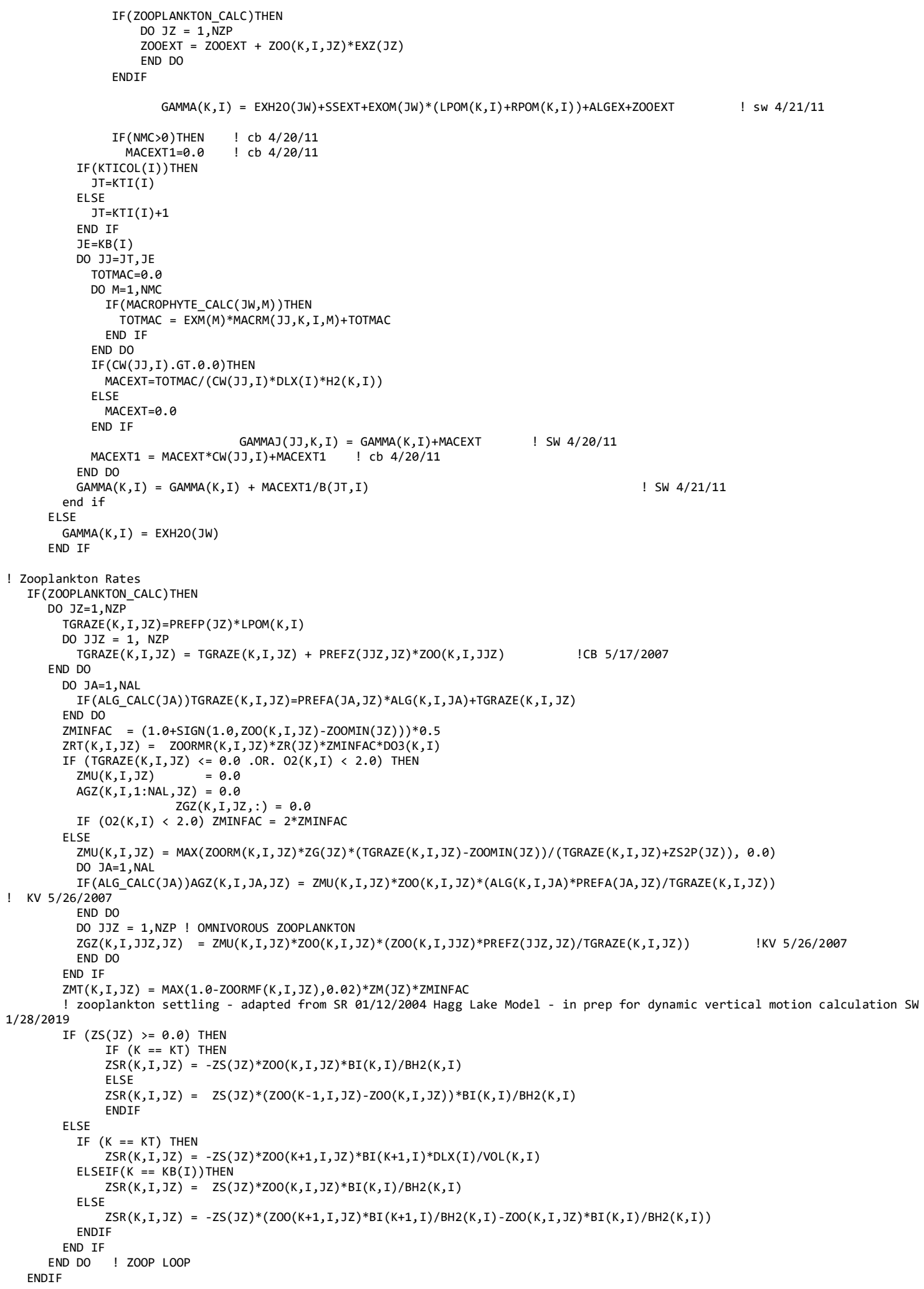




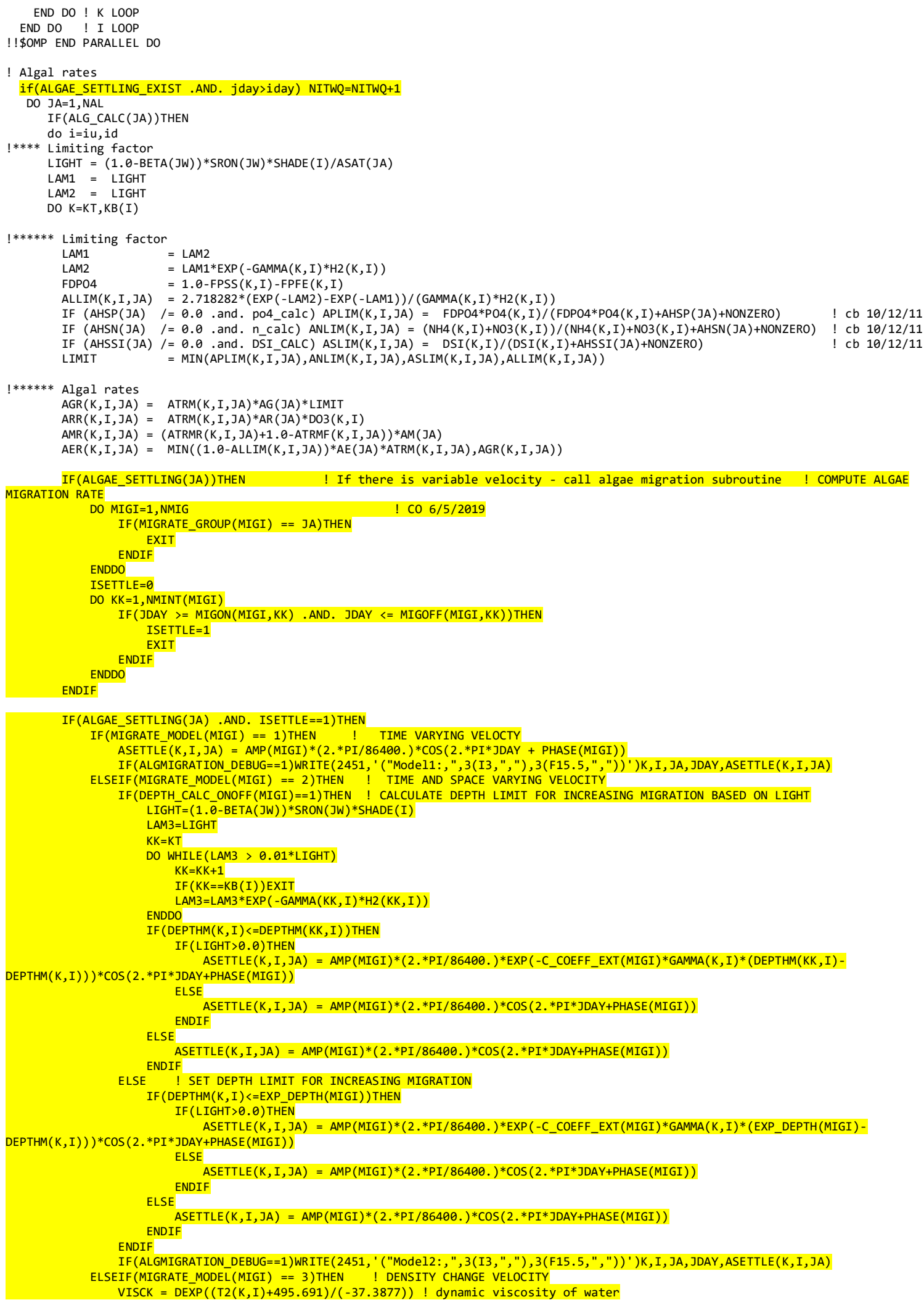




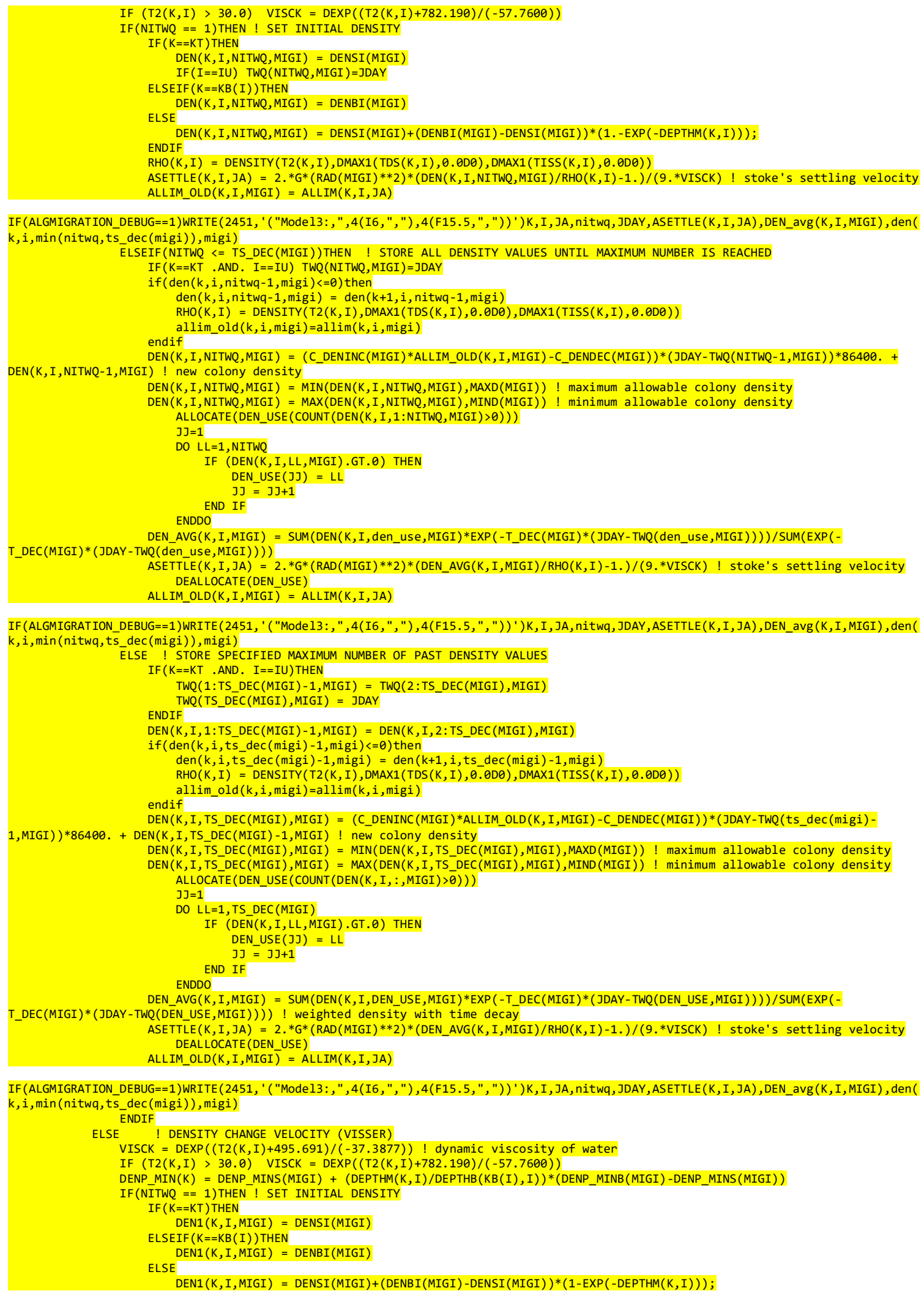




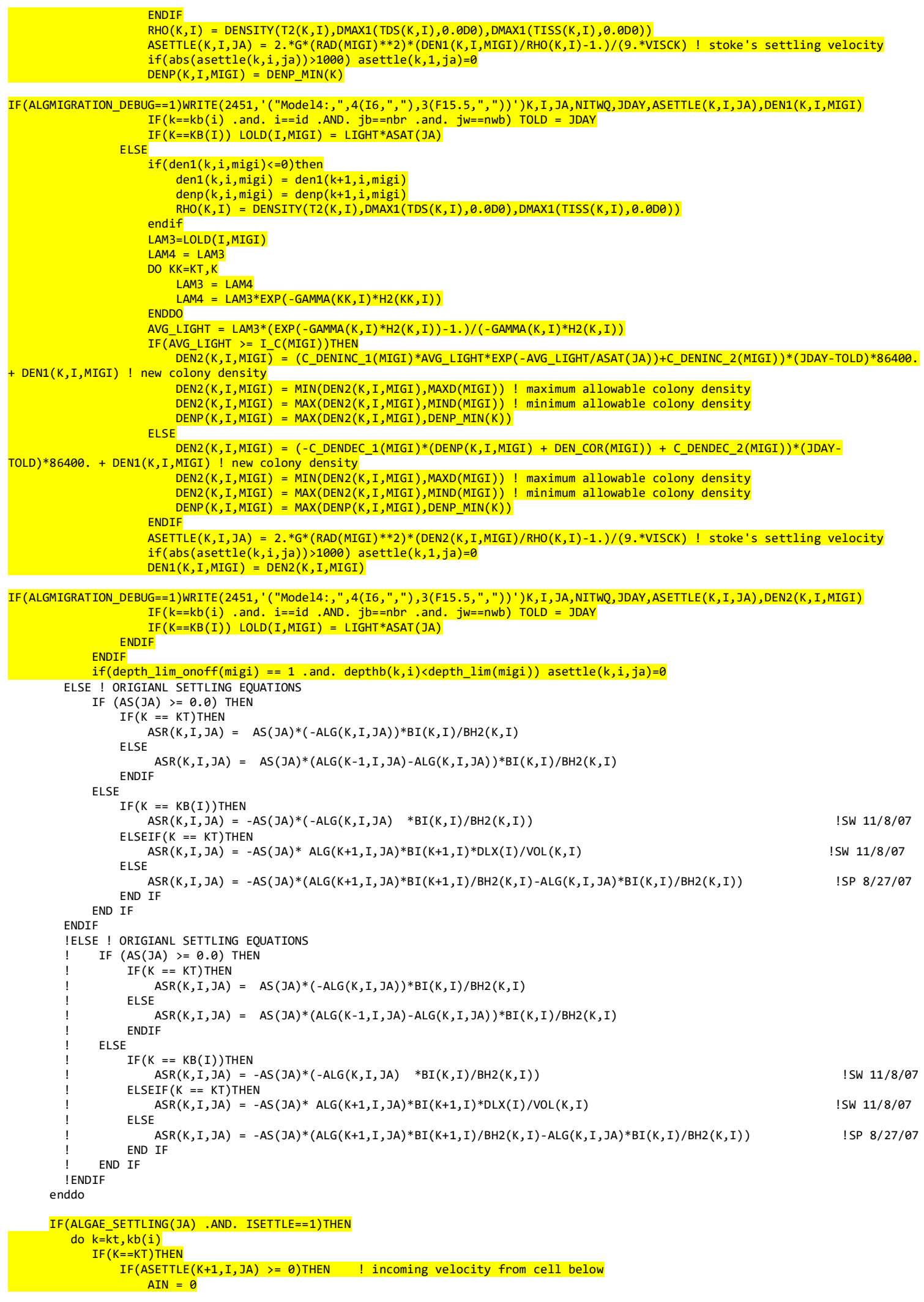




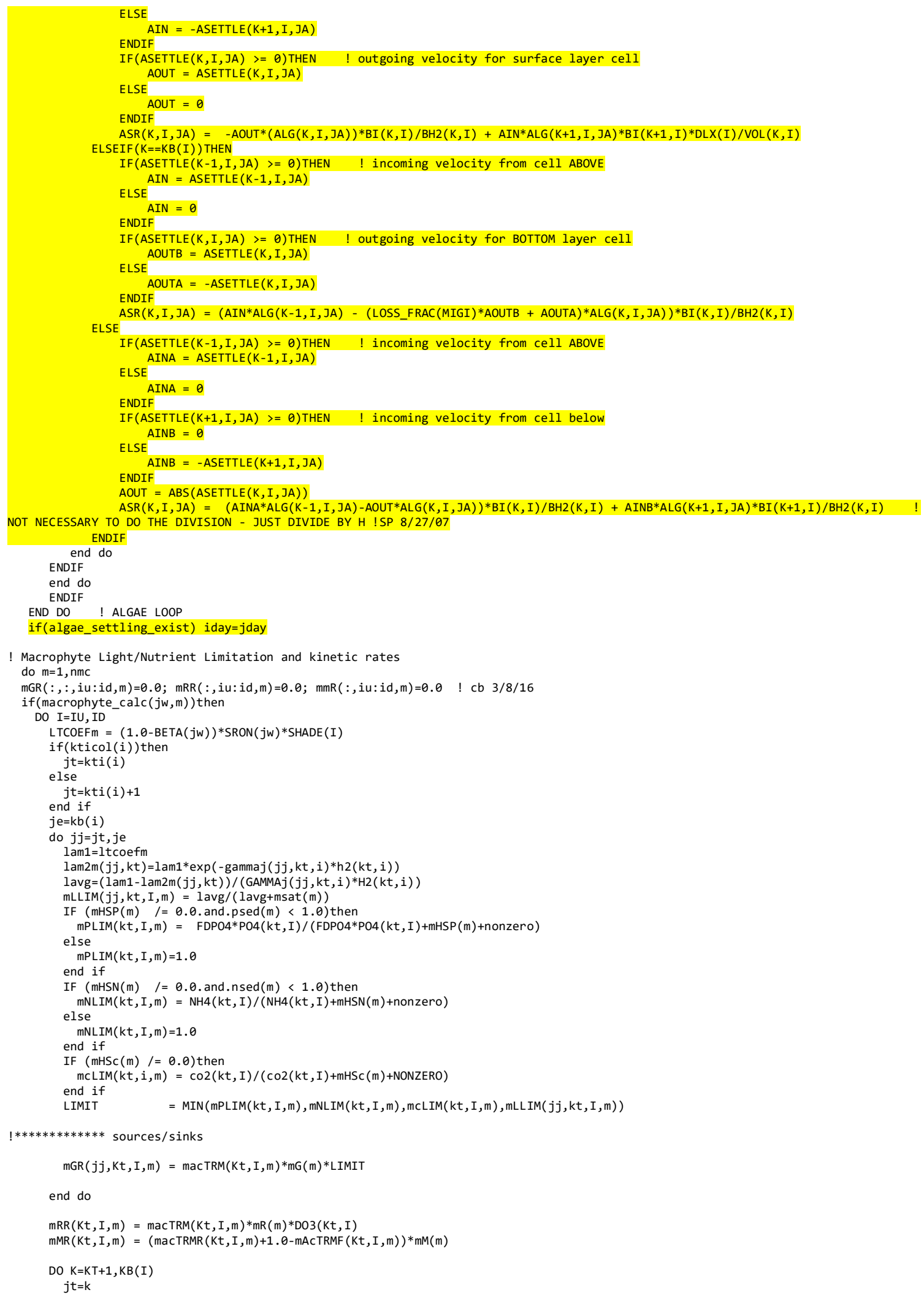




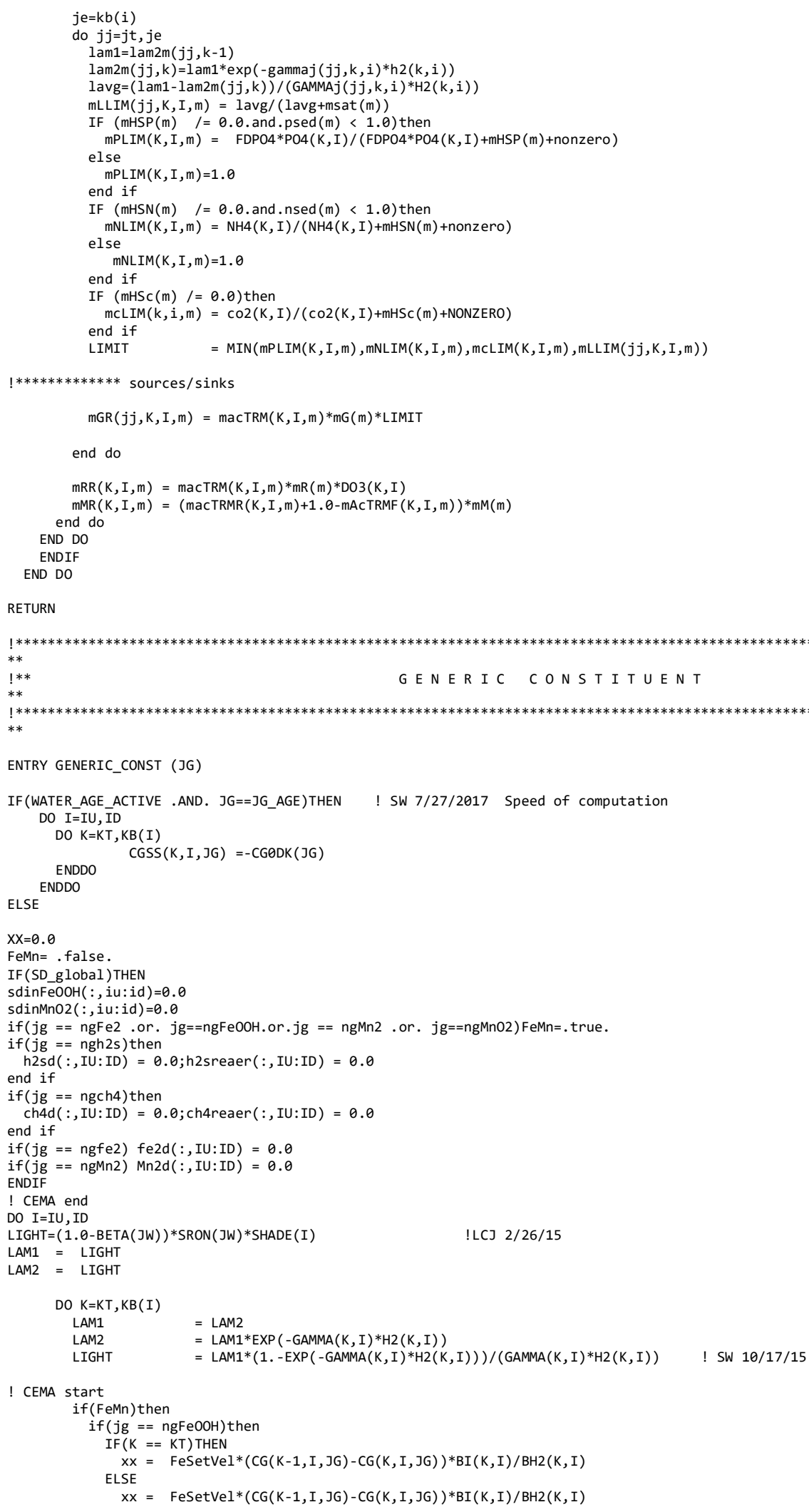




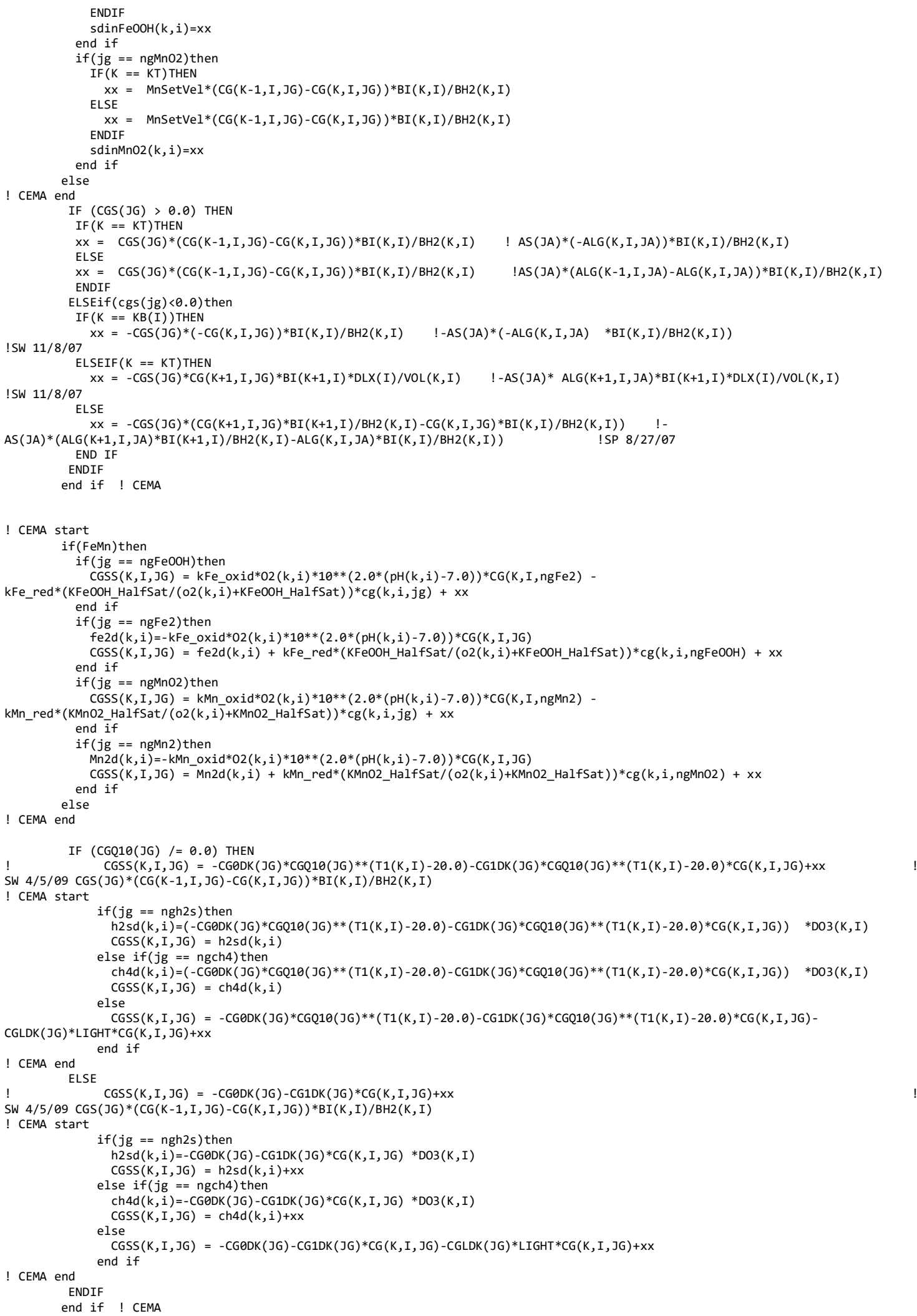




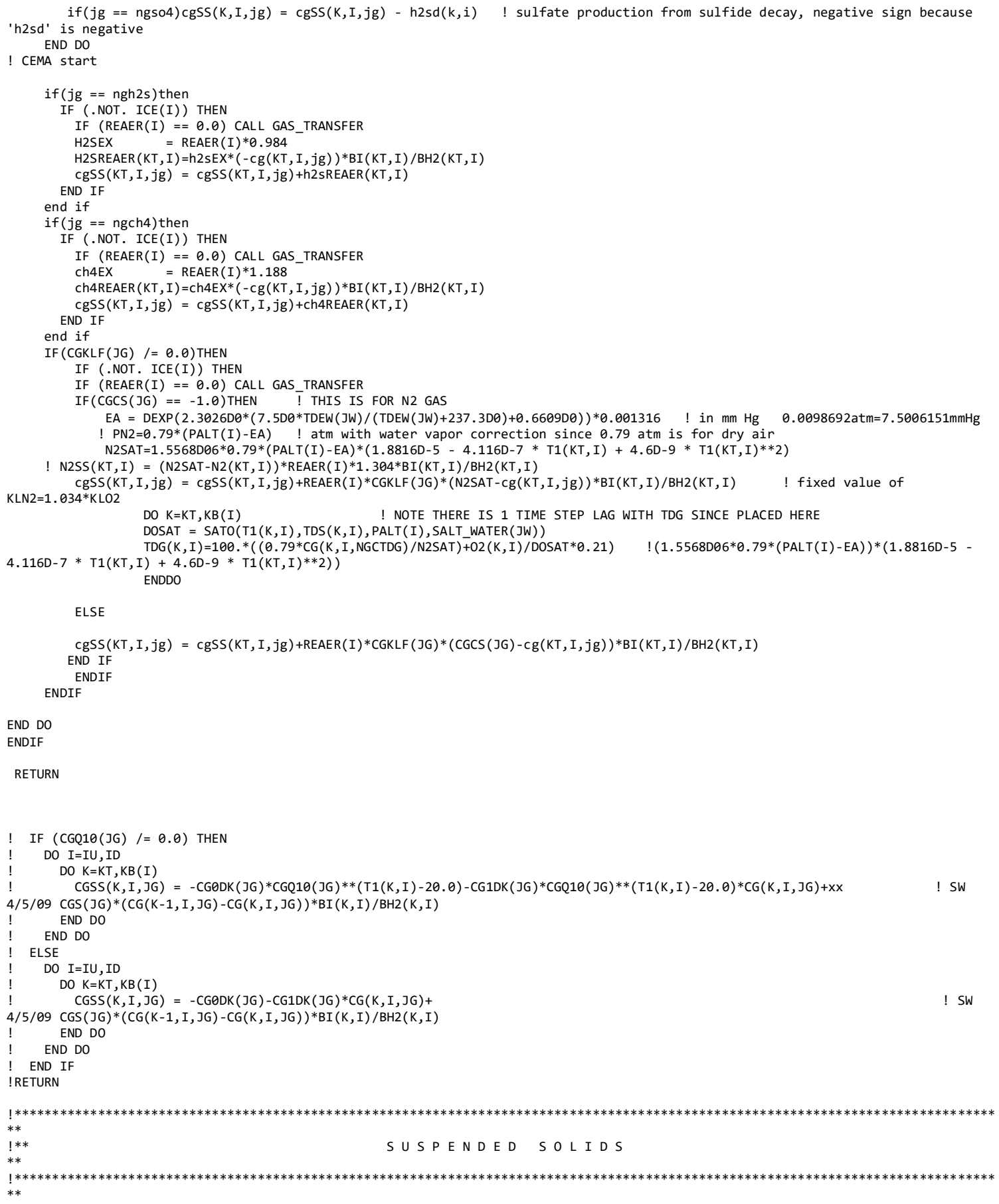

ENTRY SUSPENDED_SOLIDS (J)

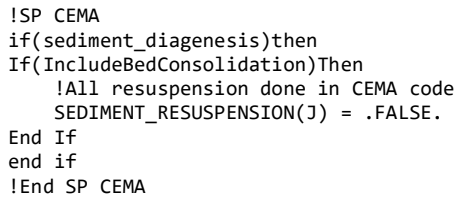




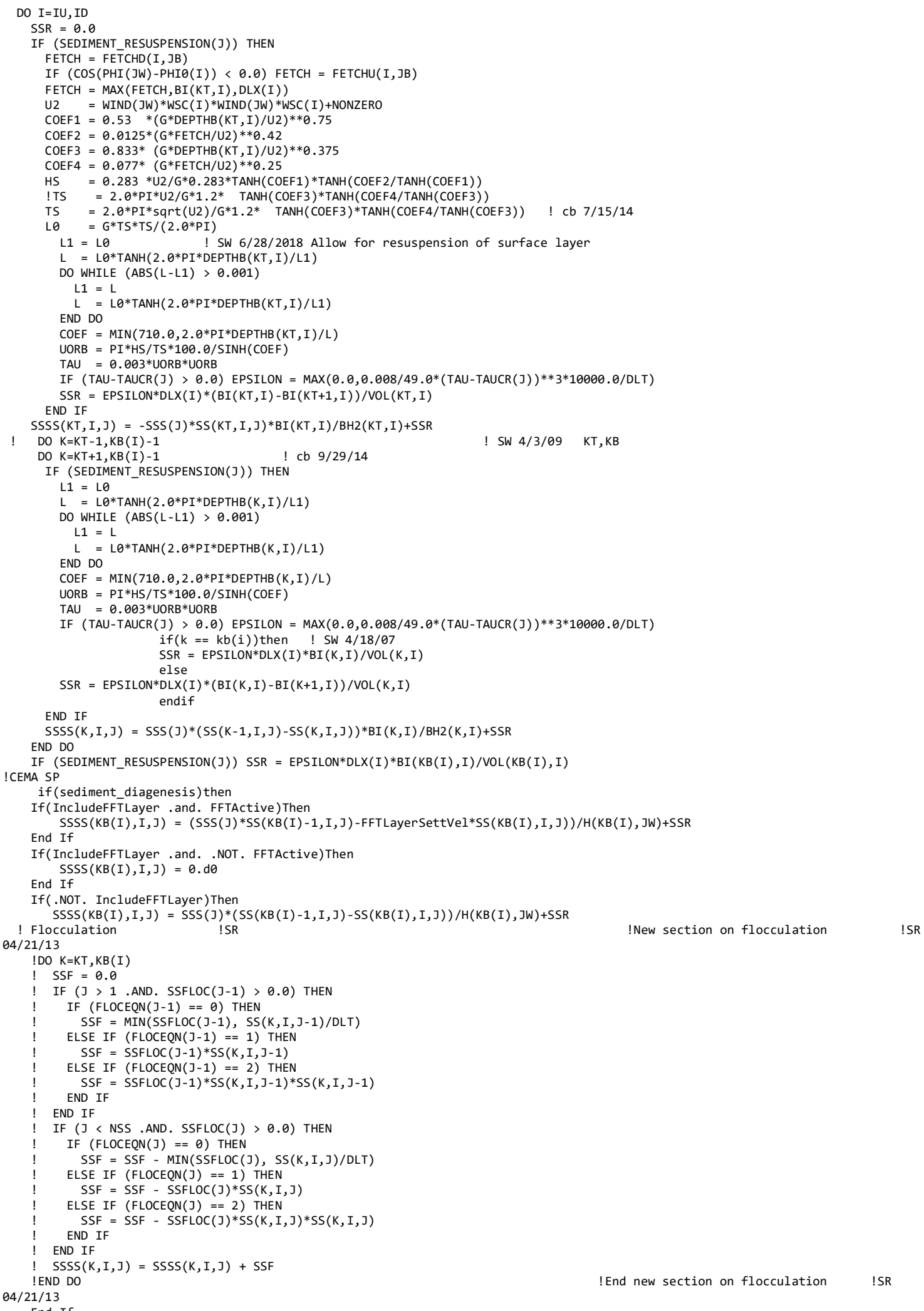

! DO K=KT, KB(I)

! $\quad \mathrm{SSF}=0.0$

IF $(J>1$.AND. $\operatorname{SSFLOC}(\mathrm{J}-1)>0.0)$ THEN

IF (FLOCEQN $(J-1)==\theta)$ THEN

SSF $=\operatorname{MIN}(\operatorname{SSFLOC}(J-1), \operatorname{SS}(K, I, J-1) / D L T)$

ELSE IF (FLOCEQN $(J-1)==1)$ THEN

SSF $=\operatorname{SSFLOC}(\mathrm{J}-1) * \mathrm{SS}(\mathrm{K}, \mathrm{I}, \mathrm{J}-1)$

SSF $=\operatorname{SSFLOC}(\mathrm{J}-1) * \operatorname{SS}(\mathrm{K}, \mathrm{I}, \mathrm{J}-1)$
$\mathrm{ELSE}$ IF $(\operatorname{FLOCEQN}(\mathrm{J}-1)==2)$ THEN

$\operatorname{SSF}=\operatorname{SSFLOC}(\mathrm{J}-1) * \operatorname{SS}(\mathrm{K}, \mathrm{I}, \mathrm{J}-1) * \operatorname{SS}(\mathrm{K}, \mathrm{I}, \mathrm{J}-1)$

END IF 


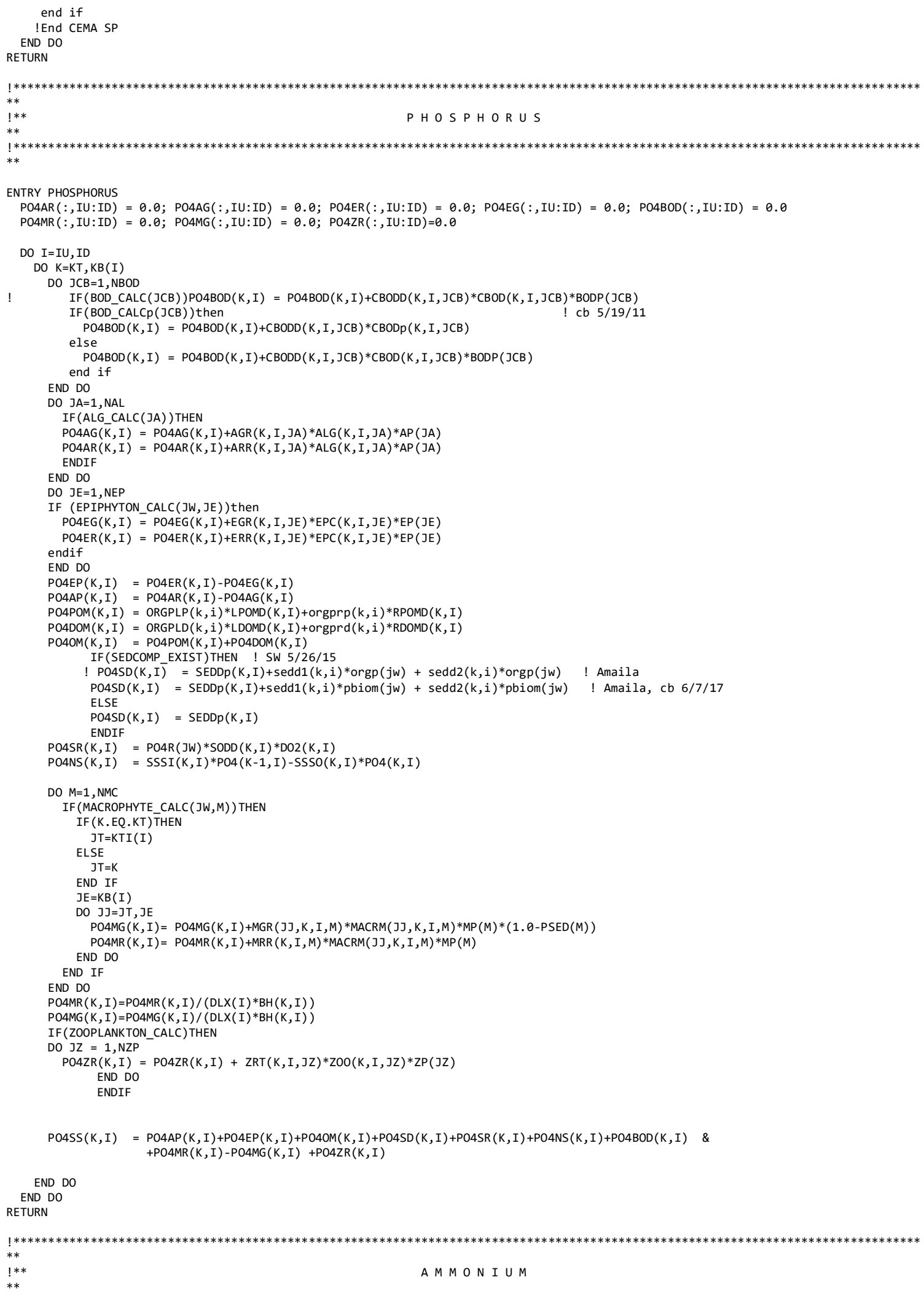




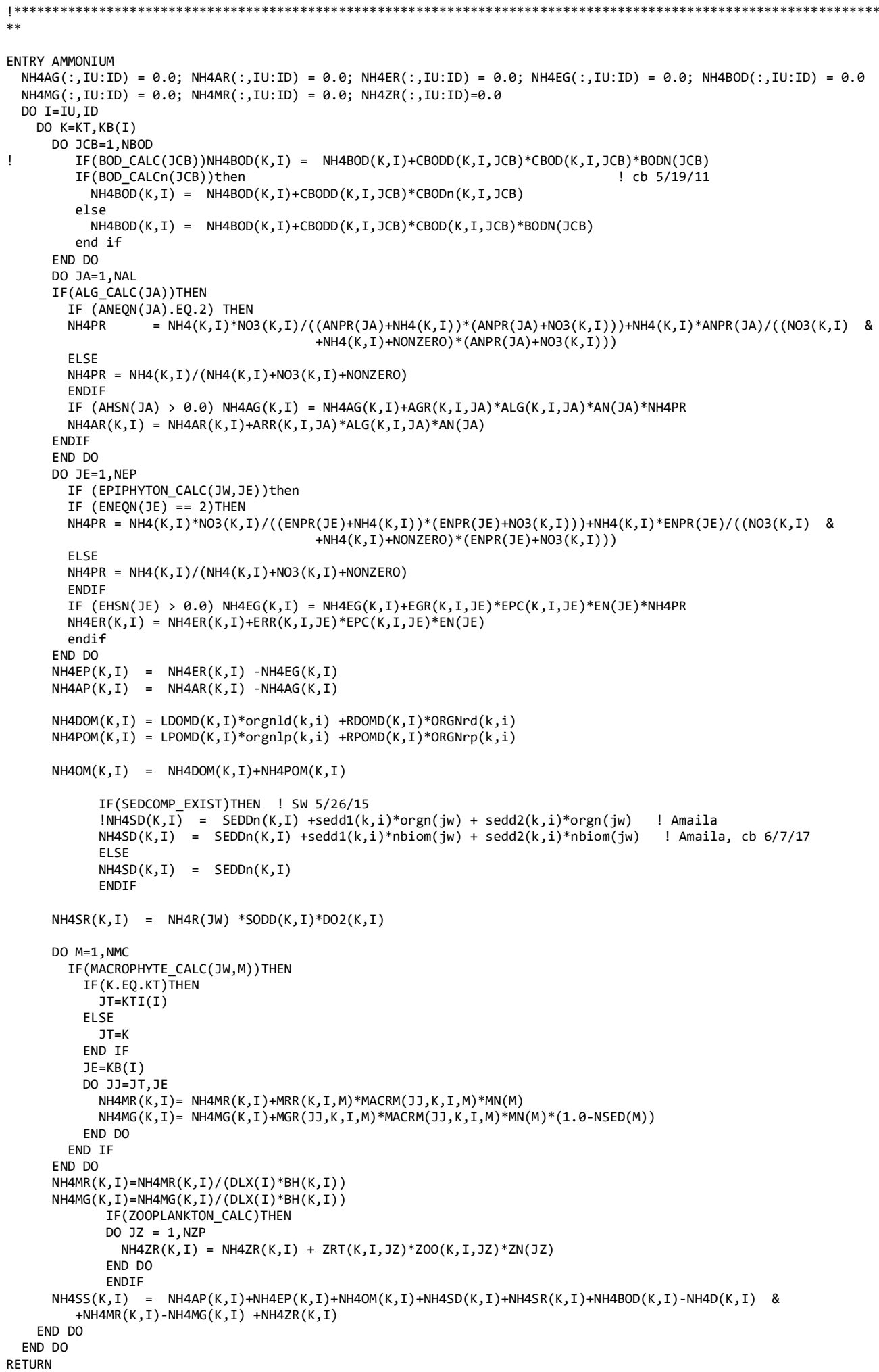




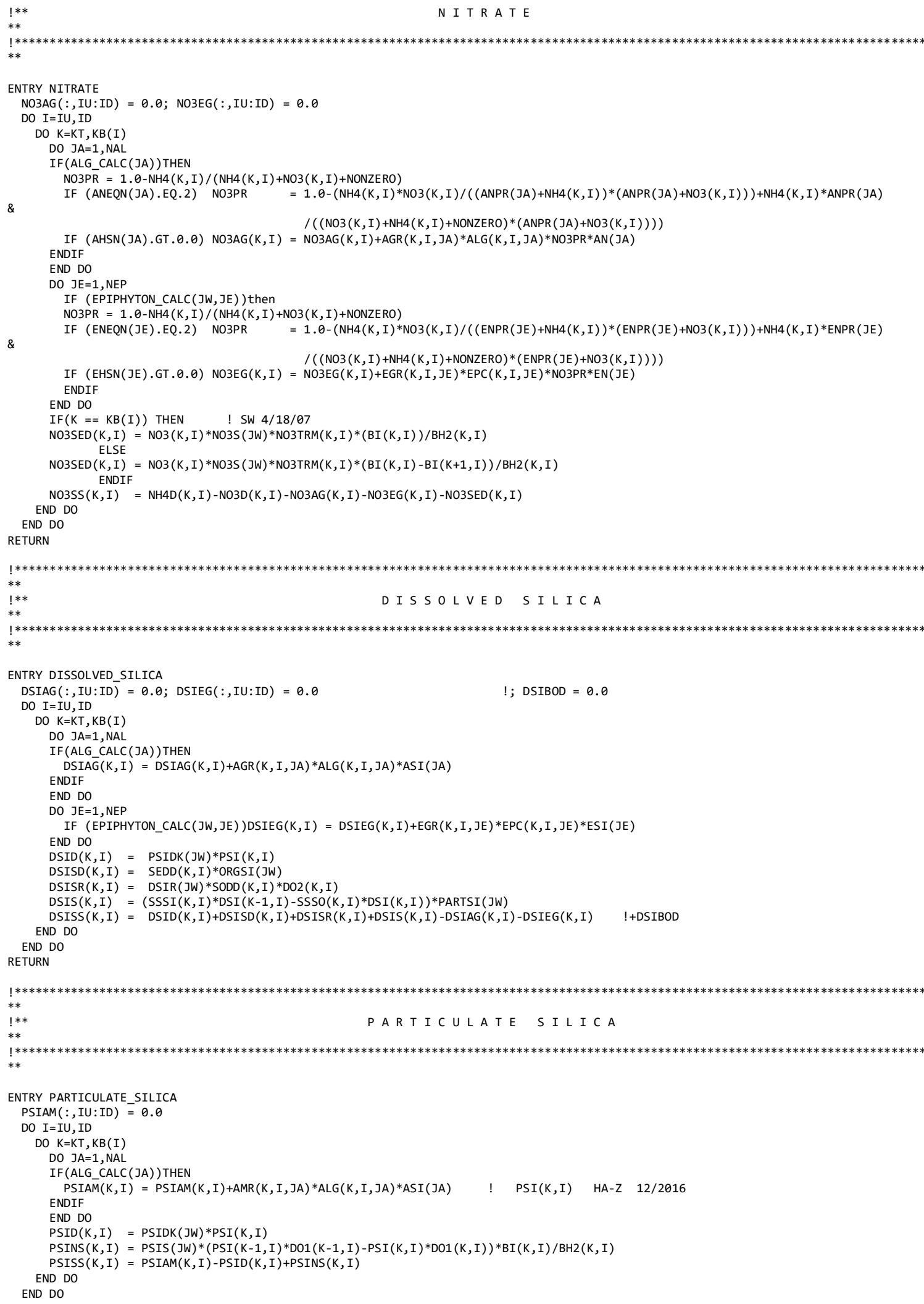




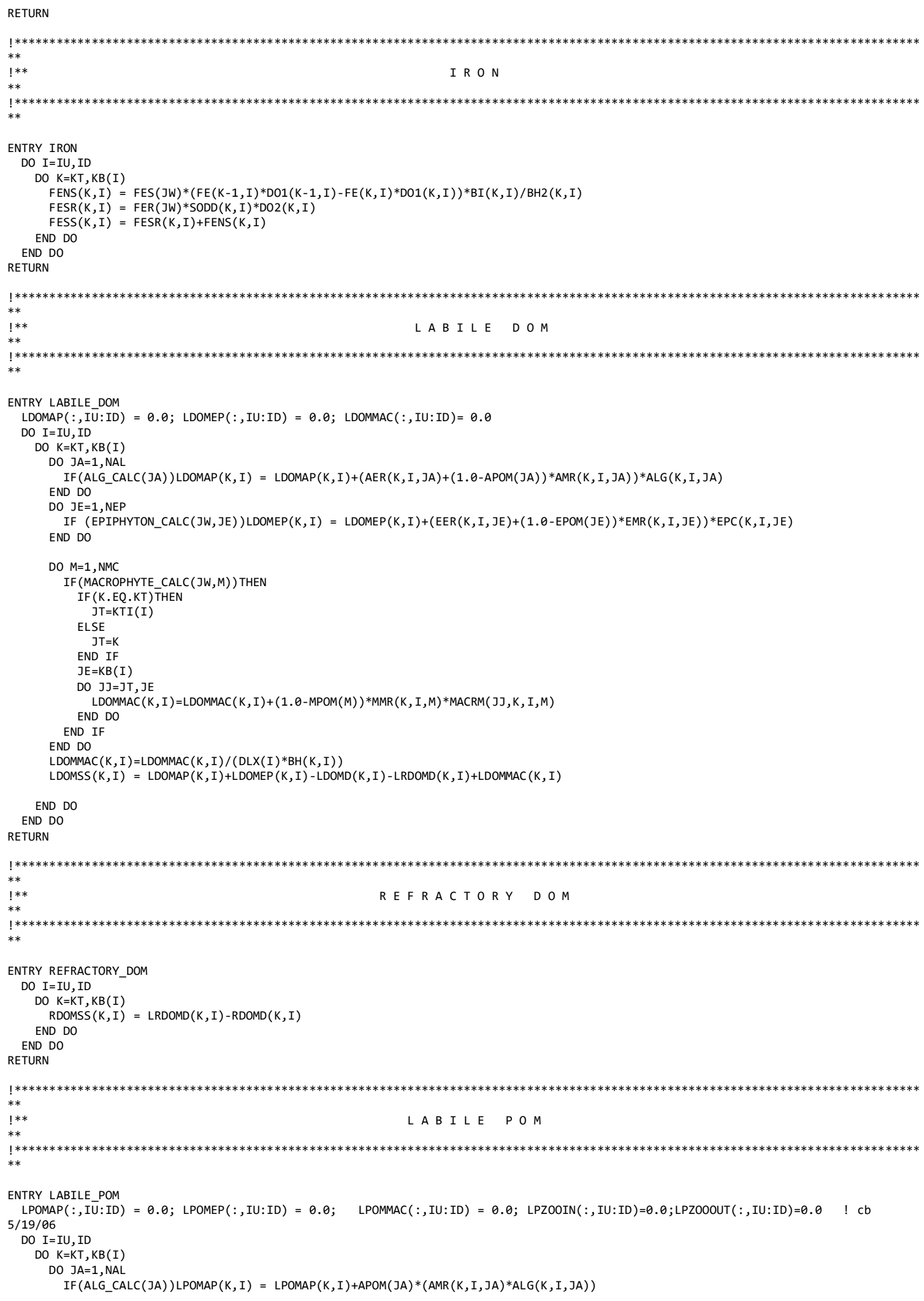




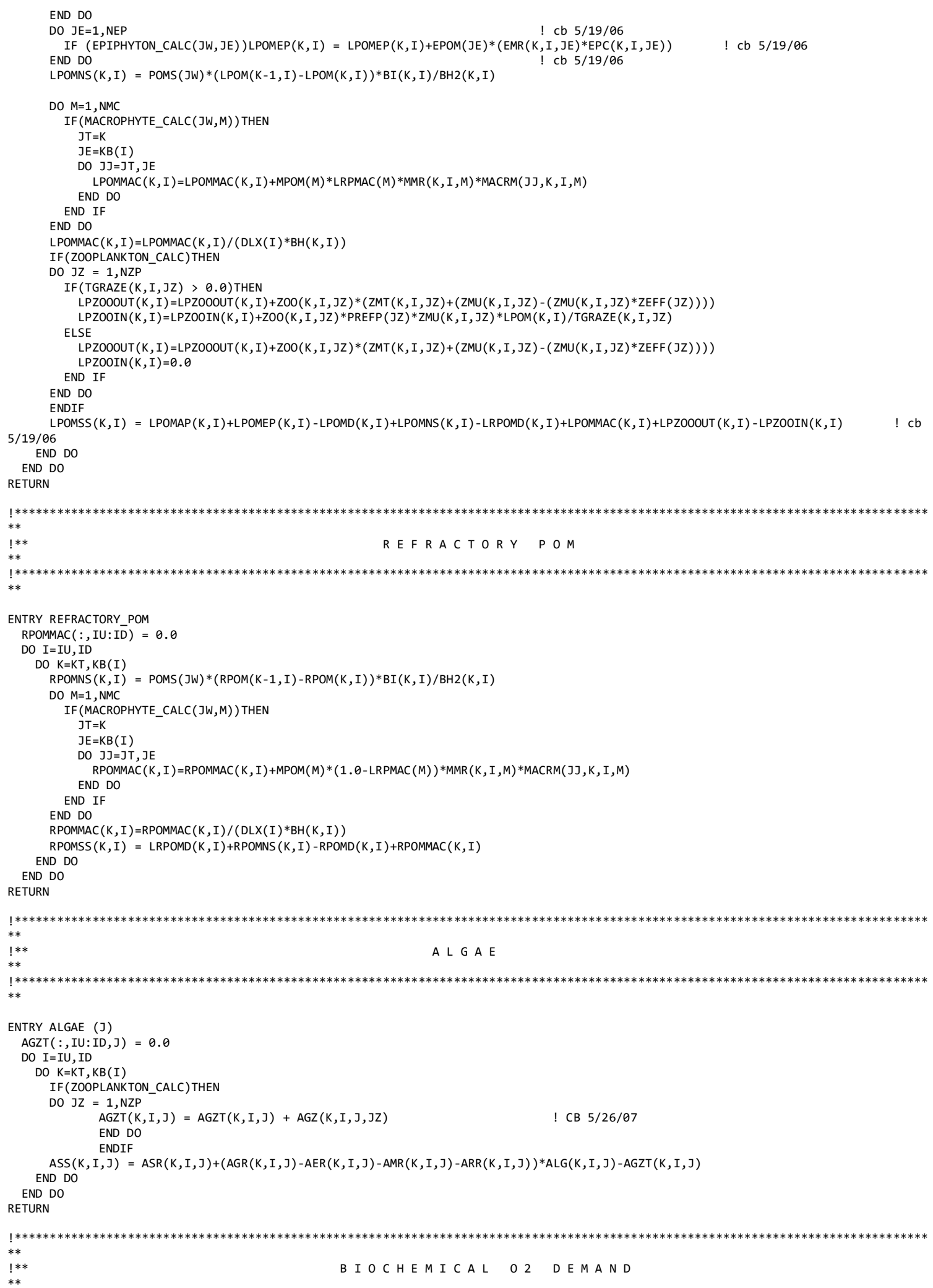




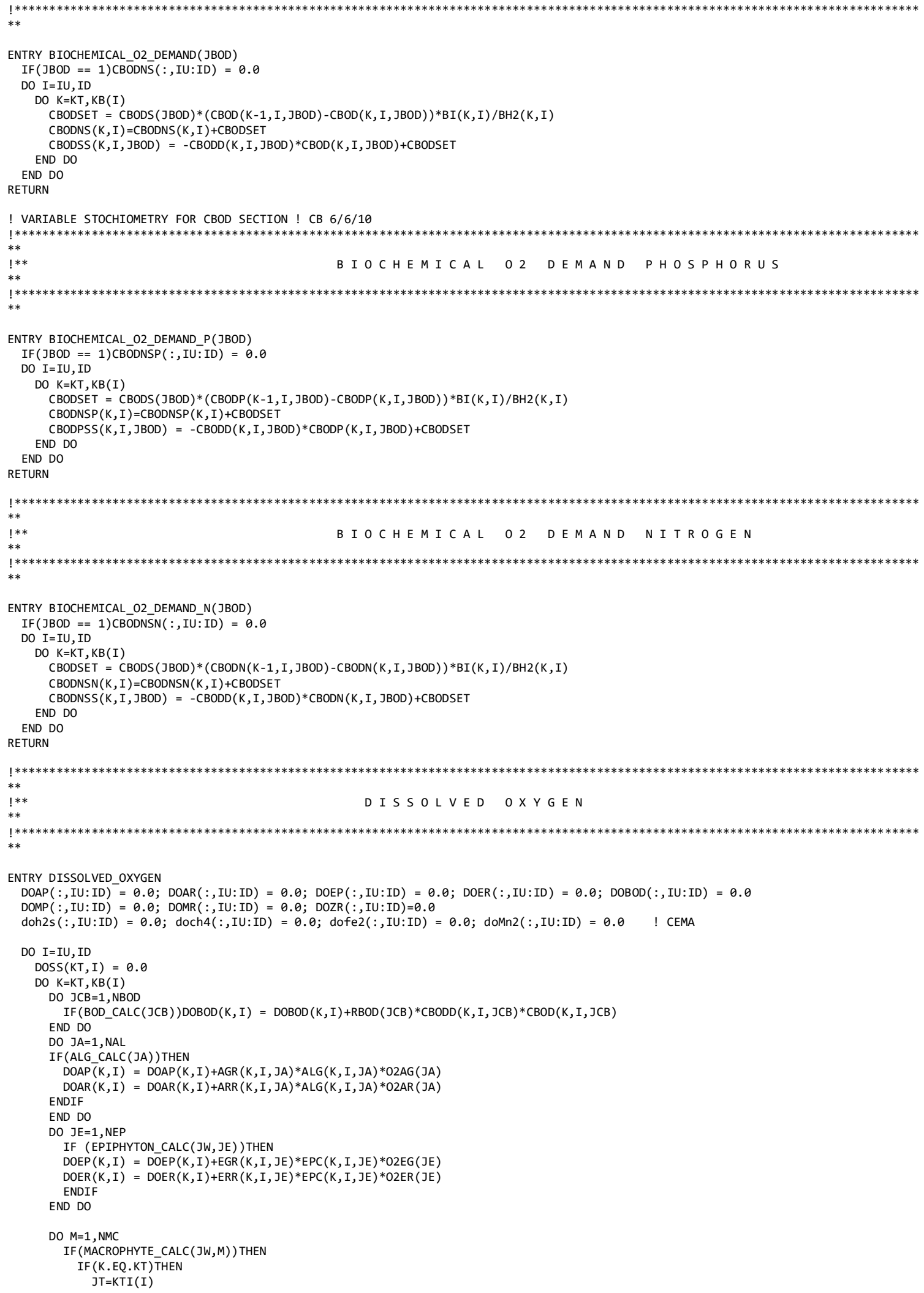




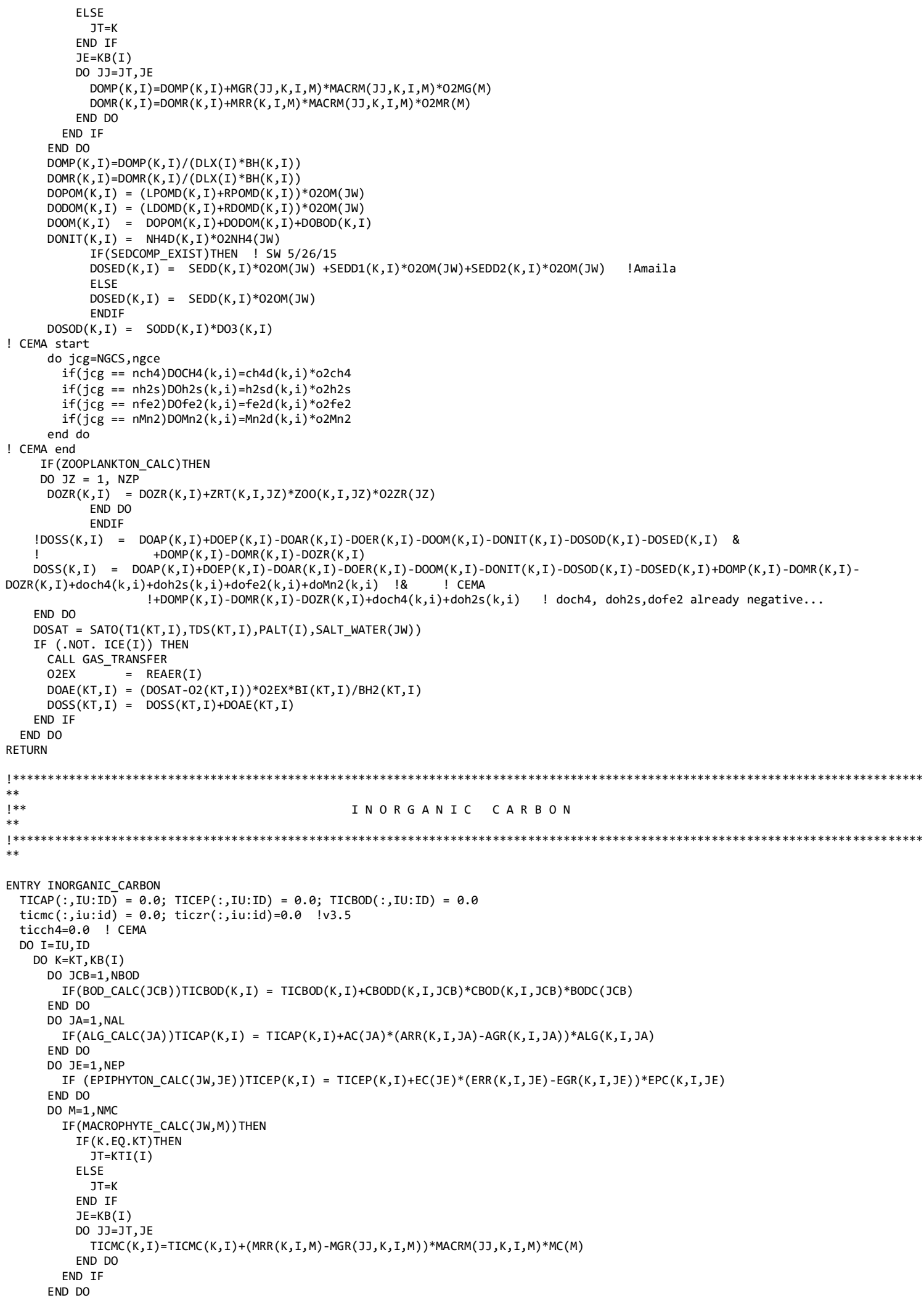




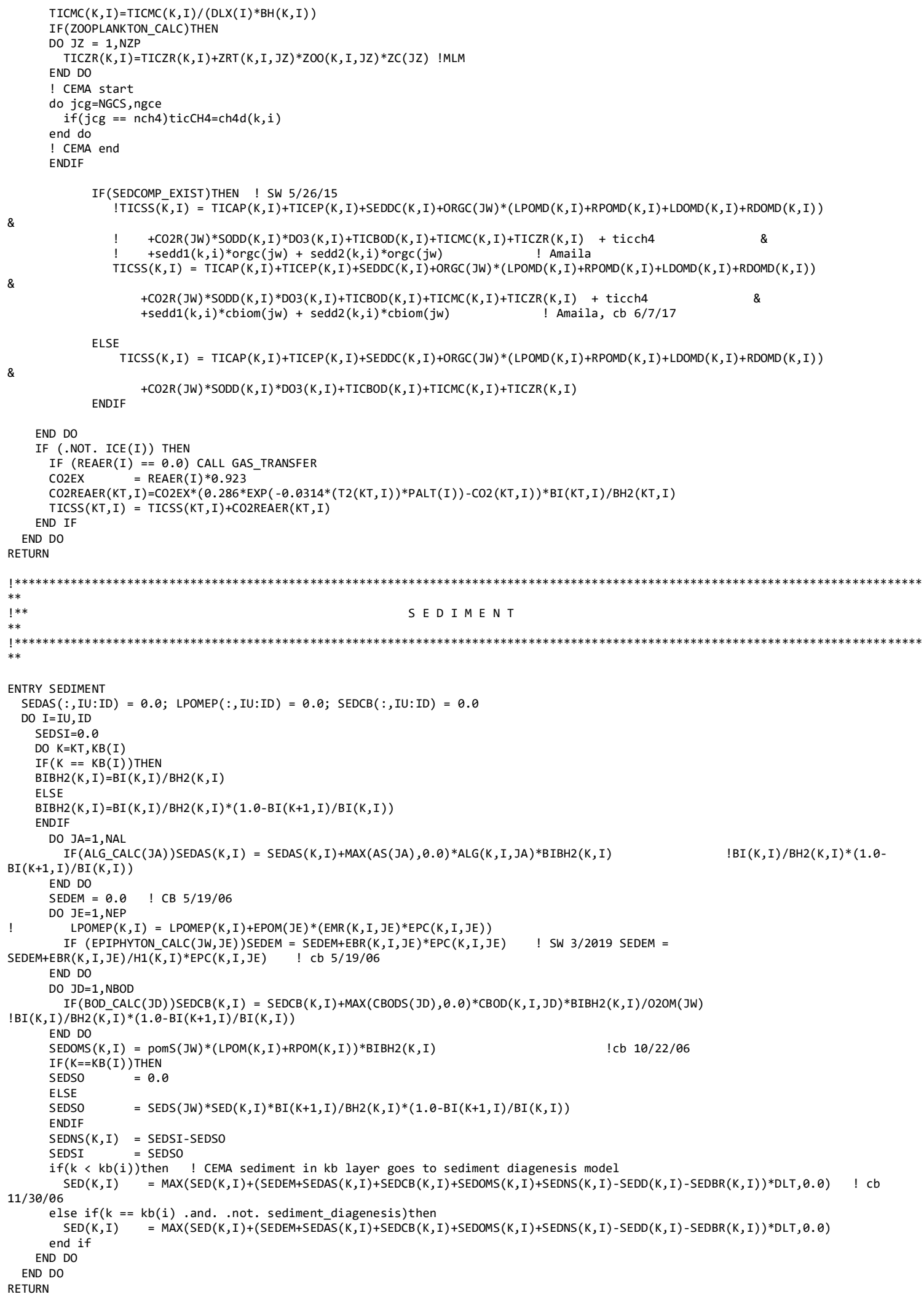




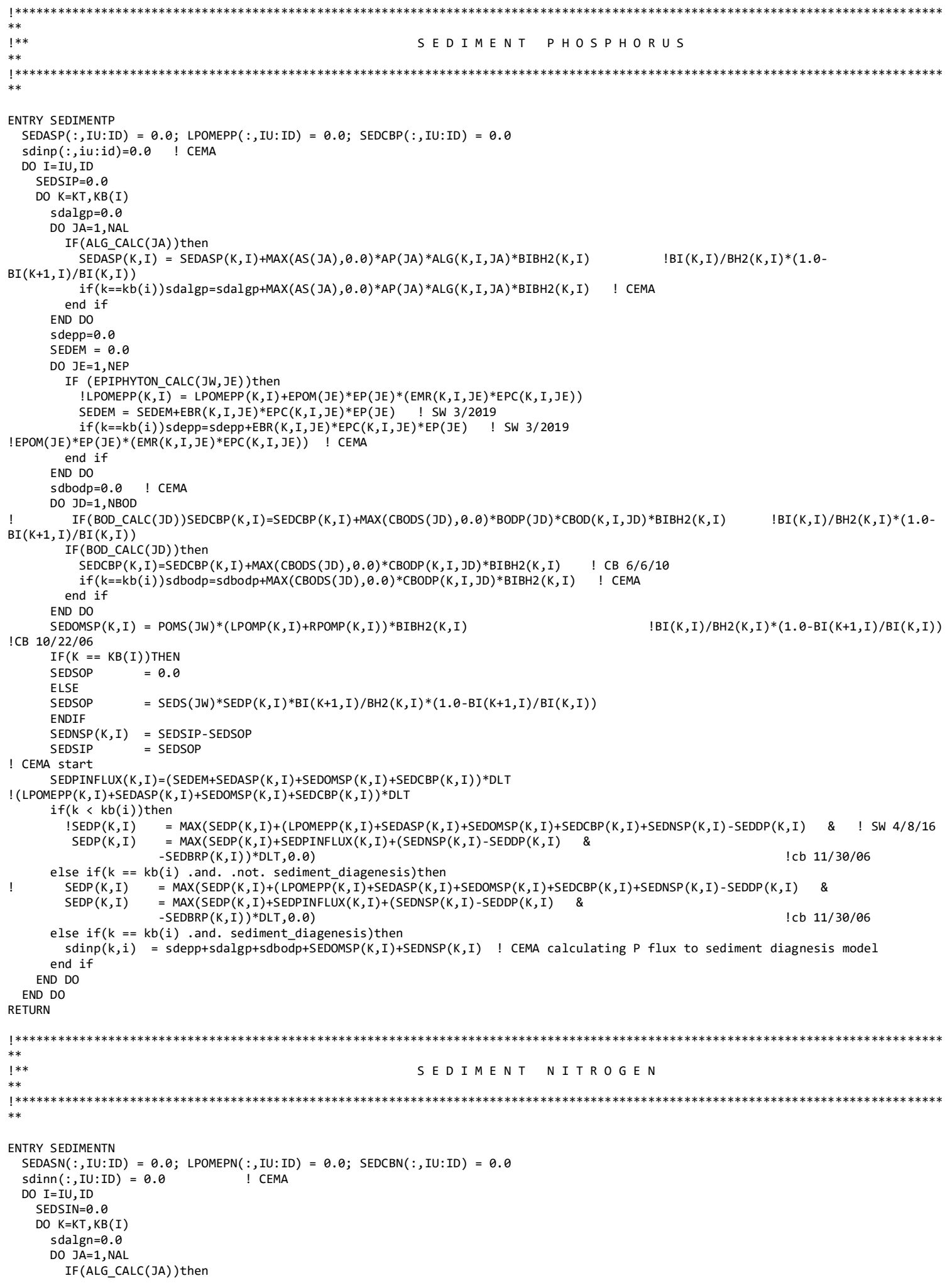




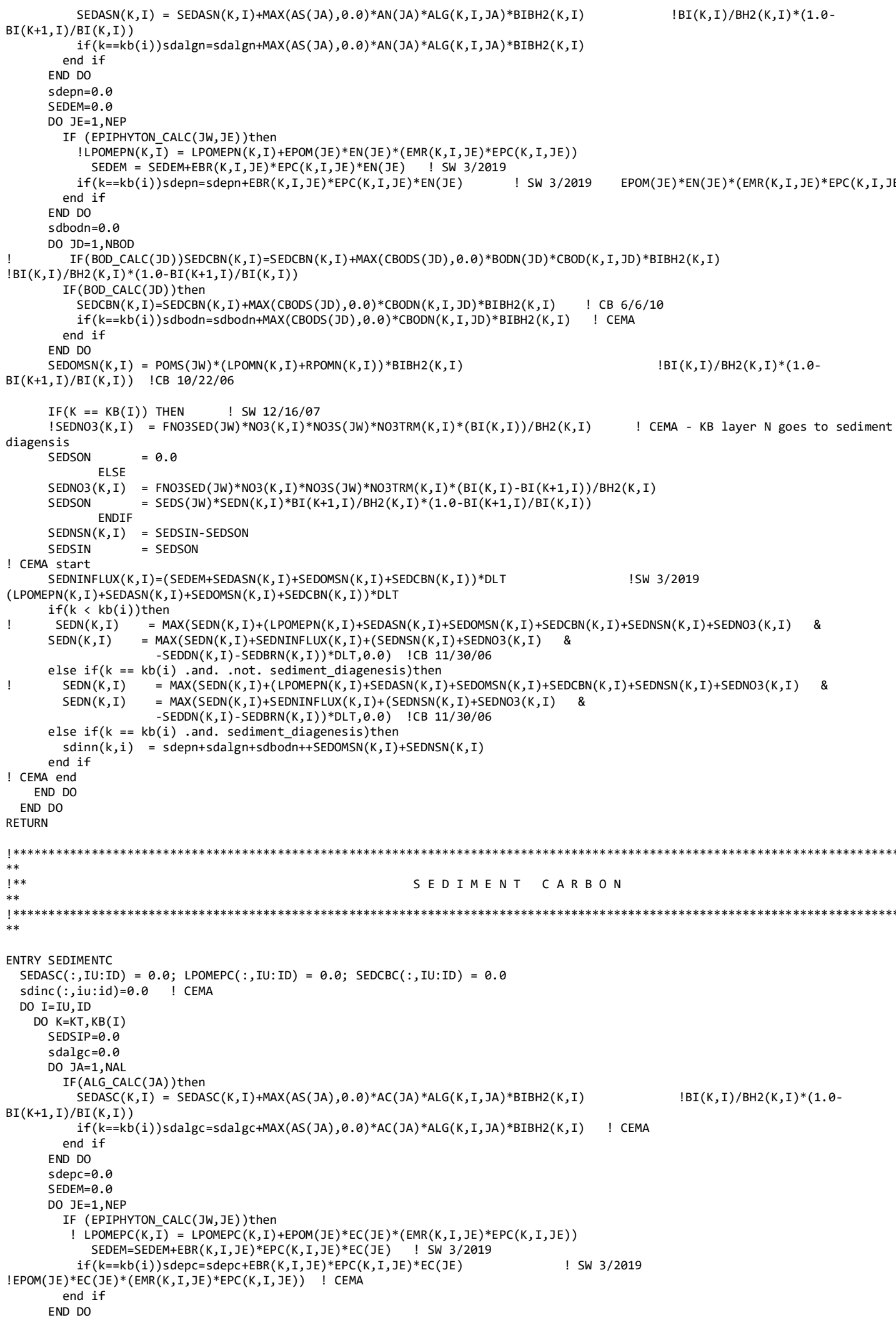




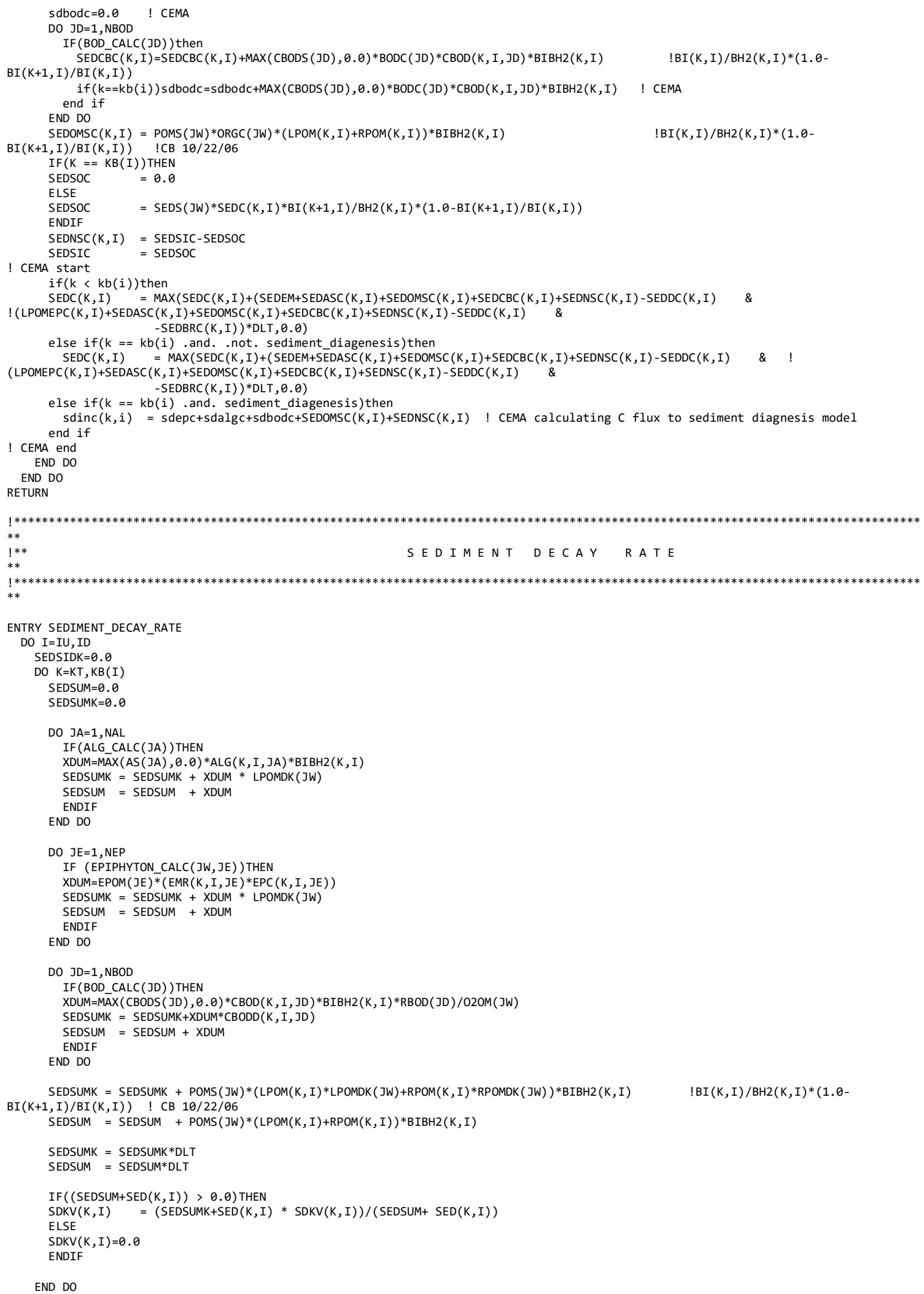




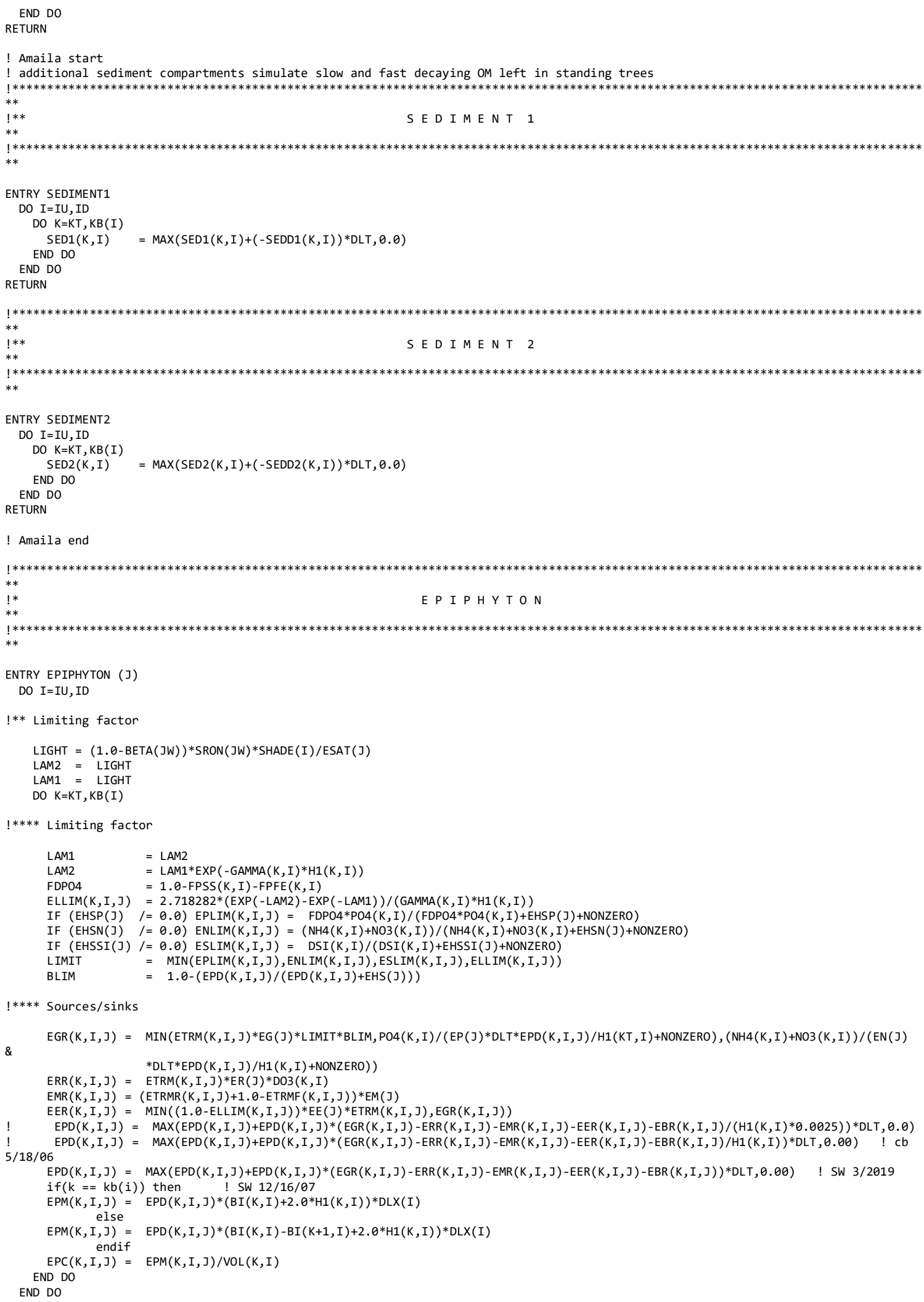




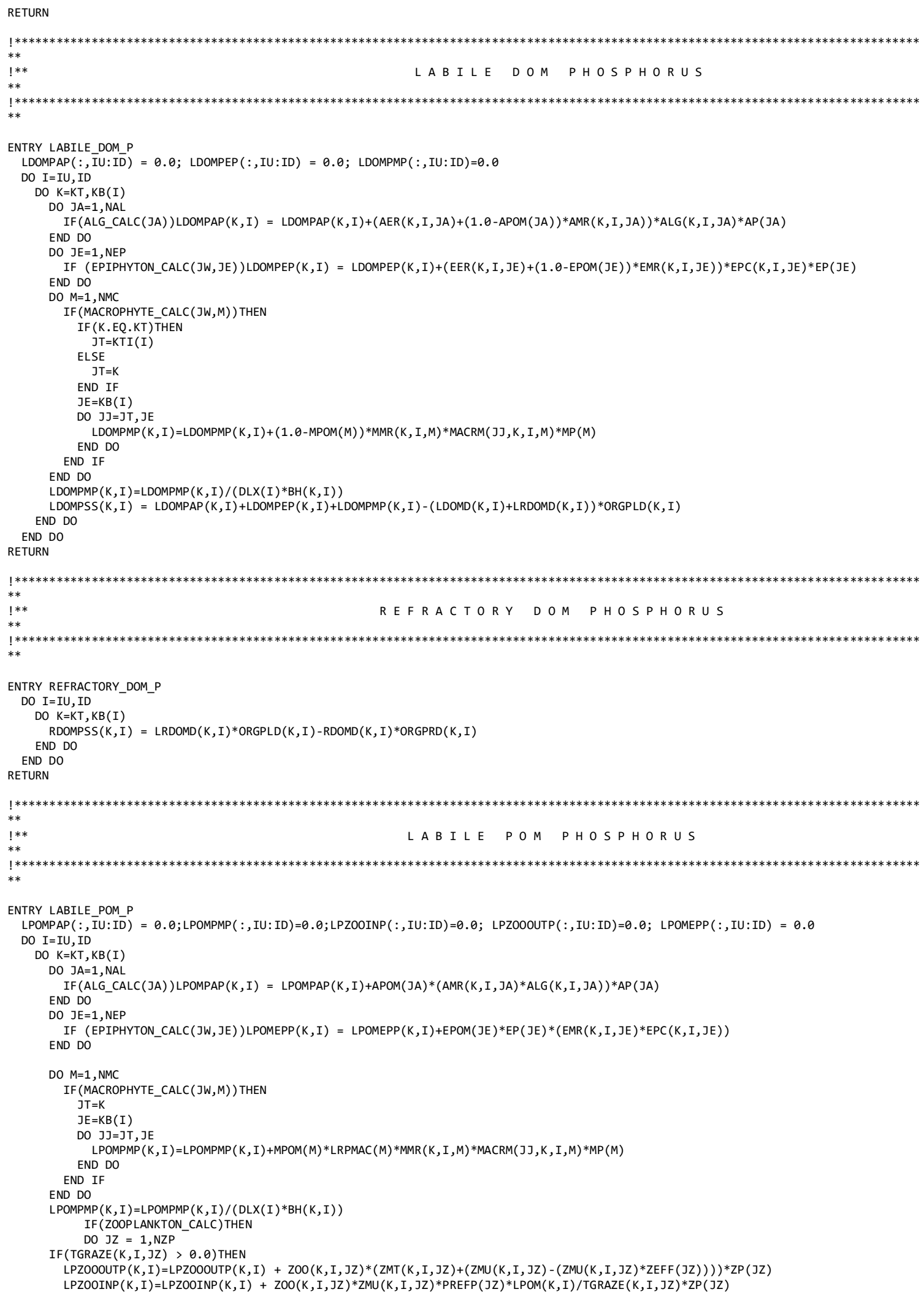

211 


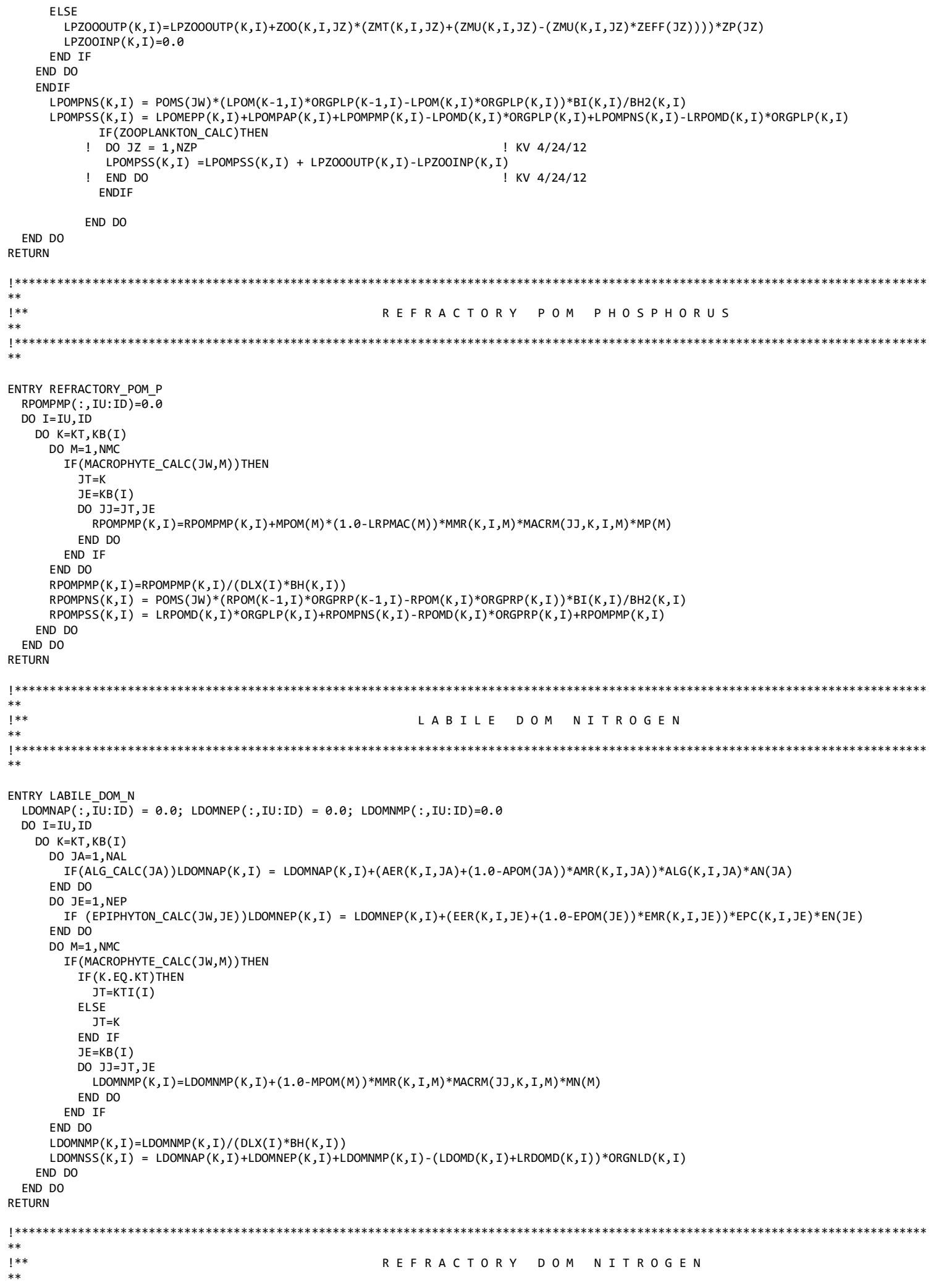




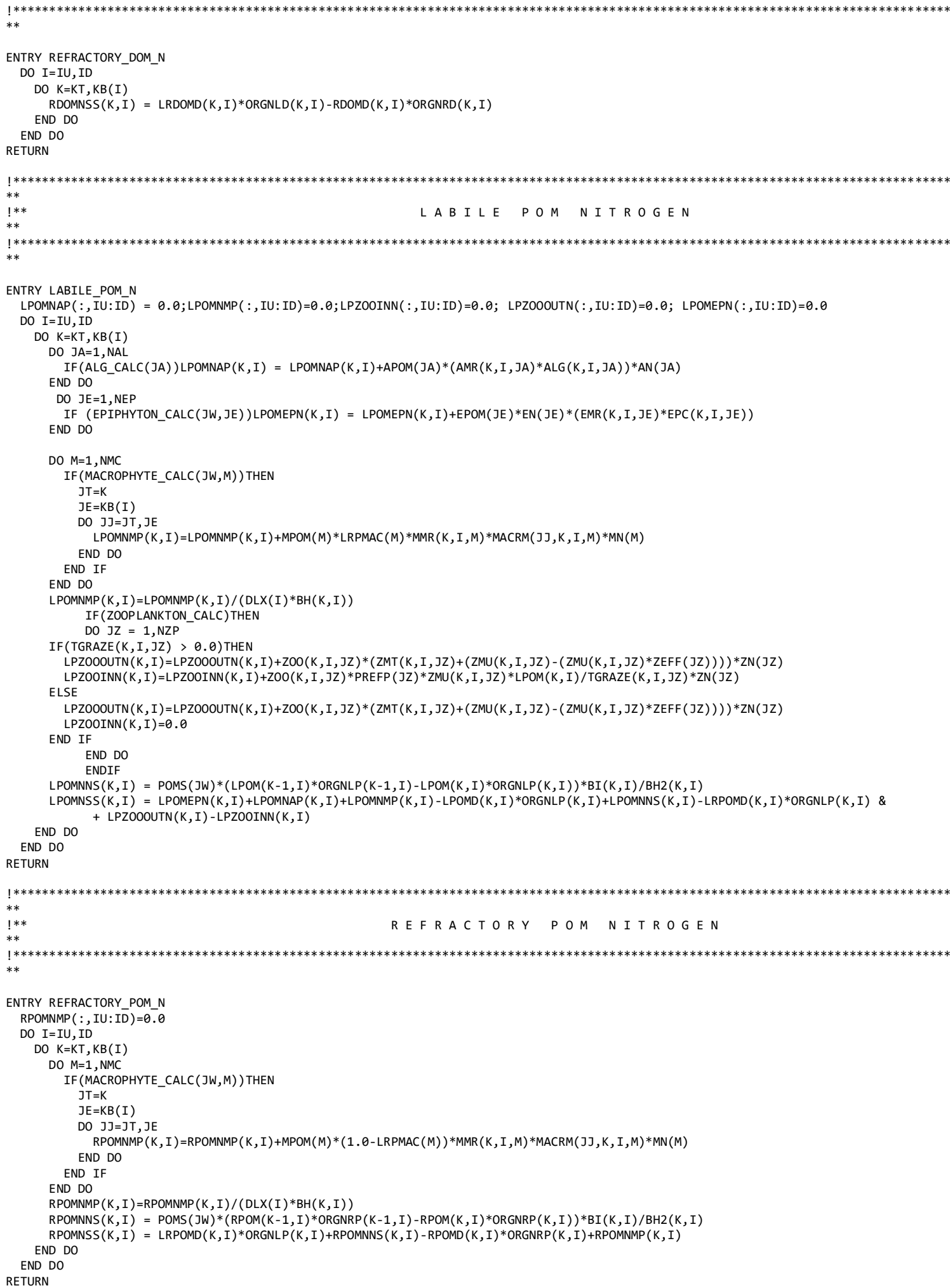




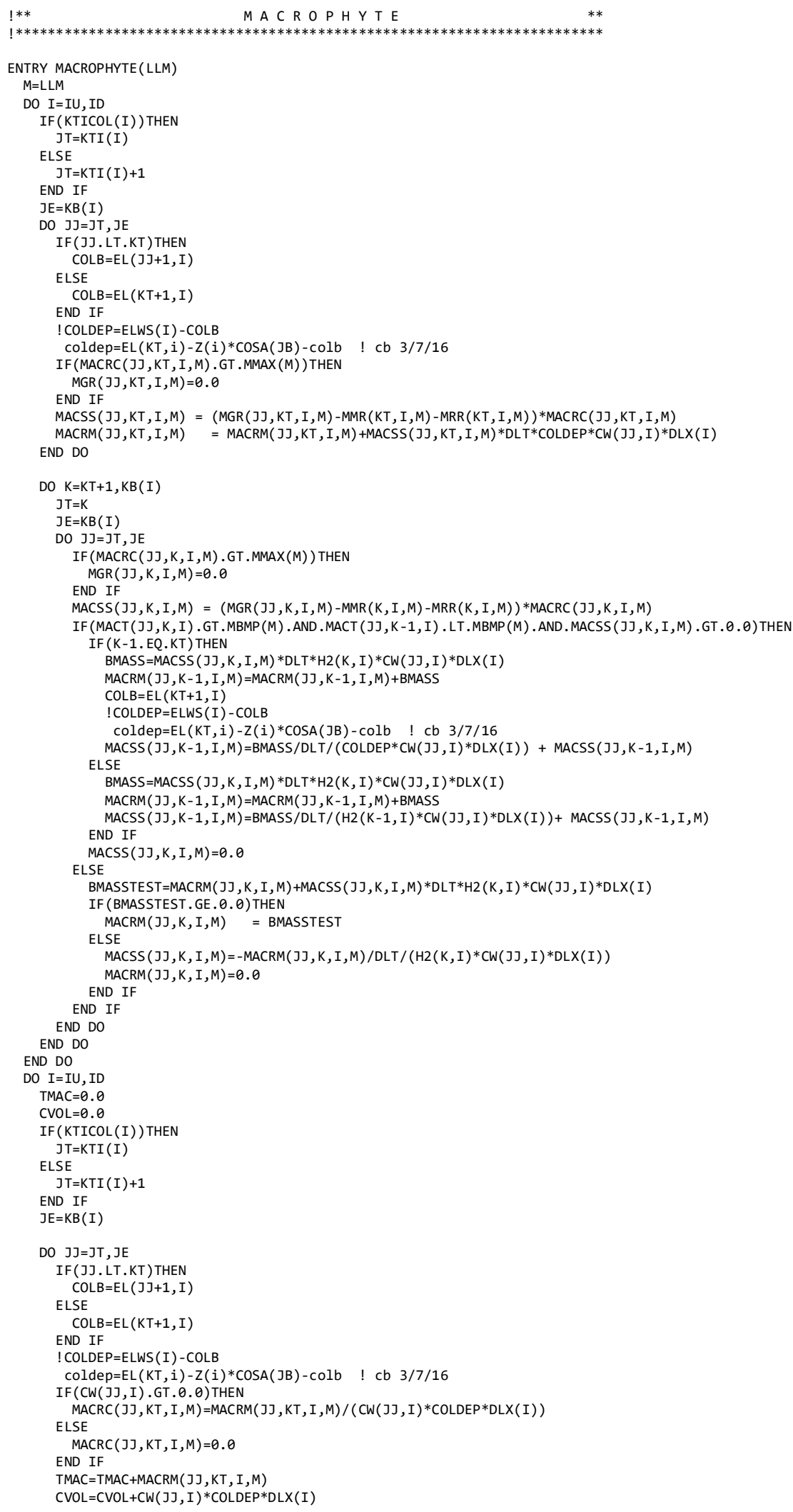




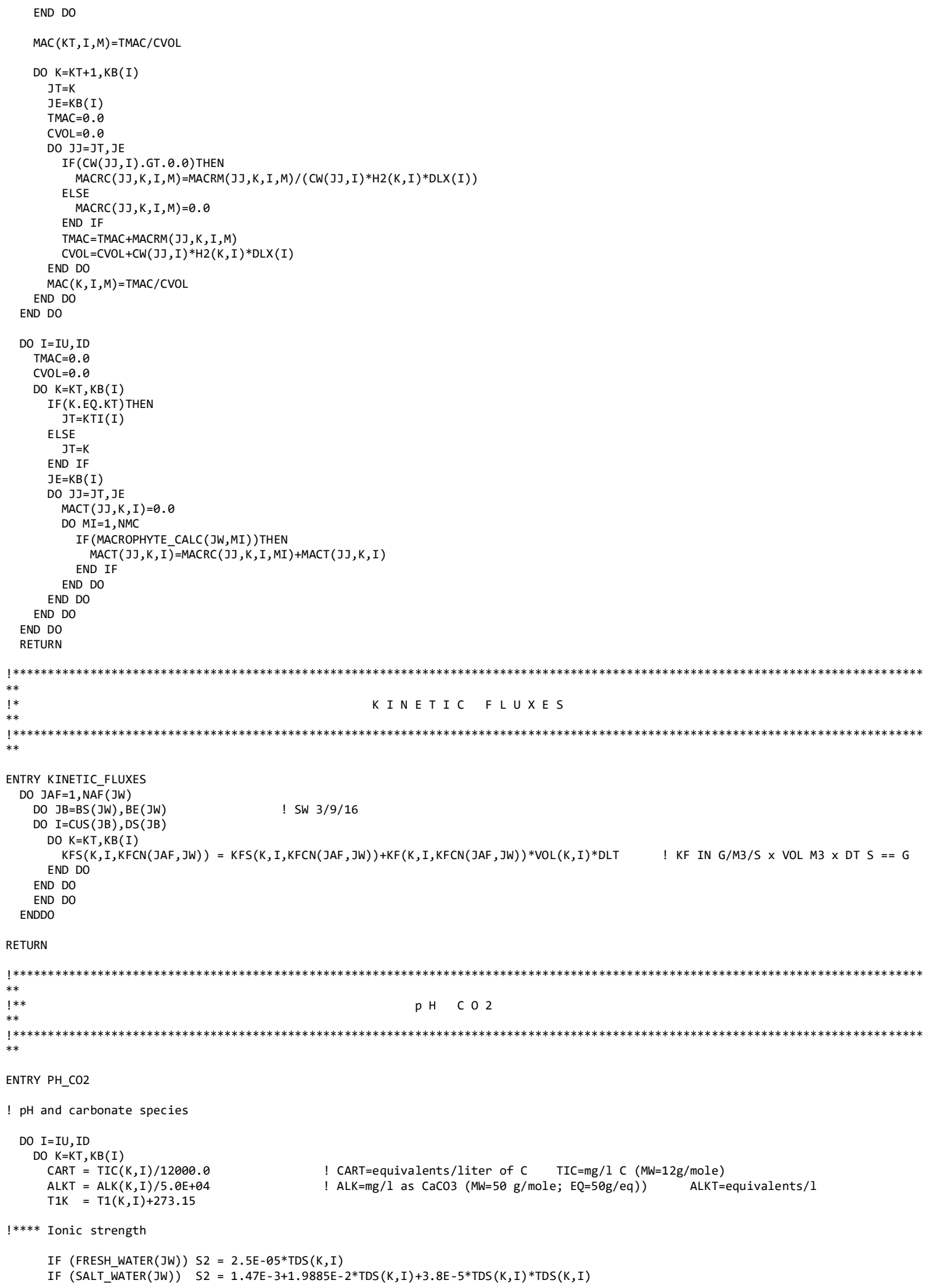


!**** Debye-Huckel terms and activity coefficients

SQRS2 $=$ SQRT $(S 2)$

$\mathrm{DH} 1=-0.5085 * \mathrm{SORS} 2 /(1.0+1.3124 * \mathrm{SORS} 2)+4.745694 \mathrm{E}-03+4.160762 \mathrm{E}-02 * \mathrm{~S} 2-9.284843 \mathrm{E}-03 * \mathrm{~S} 2 * \mathrm{~S} 2$

$\mathrm{DH} 2=-2.0340 * \mathrm{SQRS} 2 /(1 . \theta+1.4765 * \mathrm{SORS} 2)+1.205665 \mathrm{E}-02+9.715745 \mathrm{E}-02 * \mathrm{~S} 2-2.067746 \mathrm{E}-02 * \mathrm{~S} 2 * \mathrm{~S} 2$

$\mathrm{H} 2 \mathrm{CO} \mathrm{T}=10.0 * *(0.0755 * \mathrm{~S} 2)$

$\mathrm{HCO3T}=10.0^{* *} \mathrm{DH}$

$\mathrm{CO} \mathrm{T}=10.0^{* *} \mathrm{DH} 2$

$\mathrm{OH}=\mathrm{HCO} \mathrm{T}$

!**** Temperature adjustment

$\mathrm{KW}=10 . \theta^{* *}(-283.971-0.05069842 * \mathrm{~T} 1 \mathrm{~K}+13323.0 / \mathrm{T} 1 \mathrm{~K}+102.24447 * \mathrm{LOG} 10(\mathrm{~T} 1 \mathrm{~K})-1119669.0 /(\mathrm{T} 1 \mathrm{~K} * \mathrm{~T} 1 \mathrm{~K})) / 0 \mathrm{H}$

$\mathrm{K} 1=10.0 * *(-3404.71 / \mathrm{T} 1 \mathrm{~K}+14.8435-0.032786 * \mathrm{~T} 1 \mathrm{~K}) * \mathrm{H} 2 \mathrm{CO} \mathrm{T} / \mathrm{HCO} \mathrm{T}$

$\mathrm{K} 2=10 . \theta^{* *}(-2902.39 / \mathrm{T} 1 \mathrm{~K}+6.4980-0.023790 * \mathrm{~T} 1 \mathrm{~K}) * \mathrm{HCO3T} / \mathrm{CO} \mathrm{T}$

!**** pH evaluation

$\mathrm{PHT}=-\mathrm{PH}(\mathrm{K}, \mathrm{I})-2.1$

IF $(\mathrm{PH}(\mathrm{K}, \mathrm{I})<=0.0)$ PHT $=-14.0$

INCR $=10.0$

DO $\mathrm{N}=1,3$

$\mathrm{F} \quad=1.0$

INCR $=$ INCR $/ 10.0$

$\mathrm{ITER}=0$

DO WHILE $(F>0.0$.AND. ITER $<12)$

$\mathrm{PHT}=\mathrm{PHT}+\mathrm{INCR}$

HION $=10.0 * * \mathrm{PHT}$

BICART $=$ CART $*$ K $1 * \mathrm{HION} /(\mathrm{K} 1 * \mathrm{HION}+\mathrm{K} 1 * \mathrm{~K} 2+\mathrm{HION} * \mathrm{HION})$

$\mathrm{F}=$ BICART $*(\mathrm{HION}+2.0 * \mathrm{~K} 2) / \mathrm{HION}+\mathrm{KW} / \mathrm{HION}-\mathrm{ALKT}-\mathrm{HION} / \mathrm{OH}$

ITER $=$ ITER+1

END DO

PHT $=$ PHT - INCR

END DO

!**** $\mathrm{pH}$, carbon dioxide, bicarbonate, and carbonate concentrations

$\mathrm{HION}=10 \cdot 0^{* * \mathrm{PHT}}$

$\mathrm{PH}(\mathrm{K}, \mathrm{I})=-\mathrm{PHT}$

$\mathrm{CO} 2(\mathrm{~K}, \mathrm{I})=\operatorname{TIC}(\mathrm{K}, \mathrm{I}) /(1.0+\mathrm{K} 1 / \mathrm{HION}+\mathrm{K} 1 * \mathrm{~K} 2 /(\mathrm{HION} * \mathrm{HION})) \quad ! \mathrm{mg} / \mathrm{l}$ as C

$\mathrm{HCO3}(\mathrm{K}, \mathrm{I})=\operatorname{TIC}(\mathrm{K}, \mathrm{I}) /(1.0+\mathrm{HION} / \mathrm{K} 1+\mathrm{K} 2 / \mathrm{HION}) \quad ! \mathrm{mg} / \mathrm{l}$ as $\mathrm{C}$

$\operatorname{CO3}(\mathrm{K}, \mathrm{I})=\operatorname{TIC}(\mathrm{K}, \mathrm{I}) /((\mathrm{HION} * \mathrm{HION}) /(\mathrm{K} 1 * \mathrm{~K} 2)+\mathrm{HION} / \mathrm{K} 2+1 . \theta) \quad ! \mathrm{mg} / \mathrm{l}$ as $\mathrm{C}$

END DO

RETURN

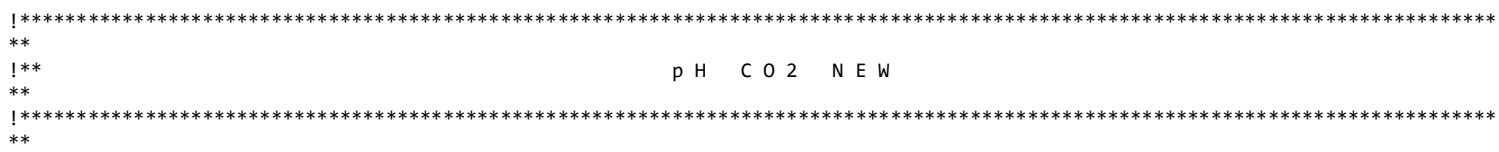

ENTRY PH_CO2_NEW ! Enhancements added for buffering by ammonia, phosphate, and OM ! SR 01/01/12

! $\mathrm{pH}$ and carbonate species

DO I=IU, ID

DO $\mathrm{K}=\mathrm{KT}, \mathrm{KB}(\mathrm{I})$

$\mathrm{T} 1 \mathrm{~K}=\mathrm{T} 1(\mathrm{~K}, \mathrm{I})+273.15$

CART $=\operatorname{TIC}(K, I) / 12011 . \quad$ SR $01 / 01 / 12$

$\operatorname{ALKT}=\operatorname{ALK}(K, I) / 50044 . \quad$ SR $01 / 01 / 12$

AMMT $=\mathrm{NH} 4(\mathrm{~K}, \mathrm{I}) / 14006.74 \quad \mathrm{SR} 01 / 01 / 12$

PHOST $=$ PO4(K,I)/30973.762! SR $01 / 01 / 12$

OMCT $=(\operatorname{LDOM}(K, I)+\operatorname{RDOM}(K, I)) * \operatorname{ORGC}(J W) / 12011$. ! moles carbon per liter from DOM ! SR 01/01/12

IF (POM_BUFFERING) OMCT $=$ OMCT $+(\operatorname{LPOM}(K, I)+R P O M(K, I)) * O R G C(J W) / 12011$. ! SR 01/01/12

!**** Ionic strength

IF (FRESH_WATER(JW)) S2 $=2.5 \mathrm{E}-05 * \operatorname{TDS}(\mathrm{K}, \mathrm{I})$

IF (SALT WATER(JW)) S2 $=1.47 \mathrm{E}-3+1.9885 \mathrm{E}-2 * \operatorname{TDS}(\mathrm{K}, \mathrm{I})+3.8 \mathrm{E}-5 * \operatorname{TDS}(\mathrm{K}, \mathrm{I}) * \operatorname{TDS}(\mathrm{K}, \mathrm{I})$

!**** Debye-Huckel terms and activity coefficients

SORS2 $=$ SORT $($ S2 $)$

$\mathrm{DH} 1=-0.5085 * \mathrm{SORS} 2 /(1.0+1.3124 * \mathrm{SORS} 2)+4.745694 \mathrm{E}-03+4.160762 \mathrm{E}-02 * \mathrm{~S} 2-9.284843 \mathrm{E}-03 * \mathrm{~S} 2 * \mathrm{~S} 2$

$\mathrm{DH} 2=-2.0340 * \mathrm{SQRS} 2 /(1.0+1.4765 * \mathrm{SQRS} 2)+1.205665 \mathrm{E}-02+9.715745 \mathrm{E}-02 * \mathrm{~S} 2-2.067746 \mathrm{E}-02 * \mathrm{~S} 2 * \mathrm{~S} 2$

$\mathrm{DH} 3=-4.5765 * \mathrm{SQRS} 2 /(1.0+1.3124 * \mathrm{SQRS} 2) !$ extended Debye-Huckel for PO4 ! SR $01 / 01 / 12$

$\mathrm{DHH}=-0.5085 * \mathrm{SQRS} 2 /(1.0+2.9529 * \mathrm{SQRS} 2)$ ! extended Debye-Huckel for $\mathrm{H}+$ ion ! SR $01 / 01 / 12$

$\mathrm{H} 2 \mathrm{CO} \mathrm{T}=10.0 * *(0.0755 * \mathrm{~S} 2)$

$\mathrm{HCO3T}=10 . \theta^{* *} \mathrm{DH} 1$

$\mathrm{CO} 3 \mathrm{~T}=10.0 * * \mathrm{DH} 2$

PO4T $=10.0^{* *}$ DH3 $!$ SR $01 / 01 / 12$

$H T=10.0^{* *}$ DHH ! activity coefficient for $\mathrm{H}+$ ! SR $01 / 01 / 12$

HPO4T = CO3T ! tabled values similar to those for carbonate ! SR $01 / 01 / 12$

$\mathrm{OHT}=\mathrm{HCO} T$ ! tabled values similar to those for bicarbonate ! SR $01 / 01 / 12$

H2PO4T = HCO3T ! tabled values similar to those for bicarbonate ! SR $01 / 01 / 12$

NH4T = HCO3T ! tabled values similar to those for bicarbonate ! SR 01/01/12

$\mathrm{NH} 3 \mathrm{~T}=\mathrm{H} 2 \mathrm{CO} 3 \mathrm{~T}$ ! neutral species, set coefficient to same as that for carbonic acid ! SR 01/01/12

H3PO4T = H2CO3T ! neutral species, set coefficient to same as that for carbonic acid ! SR 01/01/12

!**** Temperature adjustment

$\mathrm{KW}=10.0^{* *}(-283.971-0.05069842 * \mathrm{~T} 1 \mathrm{~K}+13323.0 / \mathrm{T} 1 \mathrm{~K}+102.24447 * \mathrm{LOG} 10(\mathrm{~T} 1 \mathrm{~K})-1119669.0 /(\mathrm{T} 1 \mathrm{~K} * \mathrm{~T} 1 \mathrm{~K})) / \mathrm{OHT}$

216 


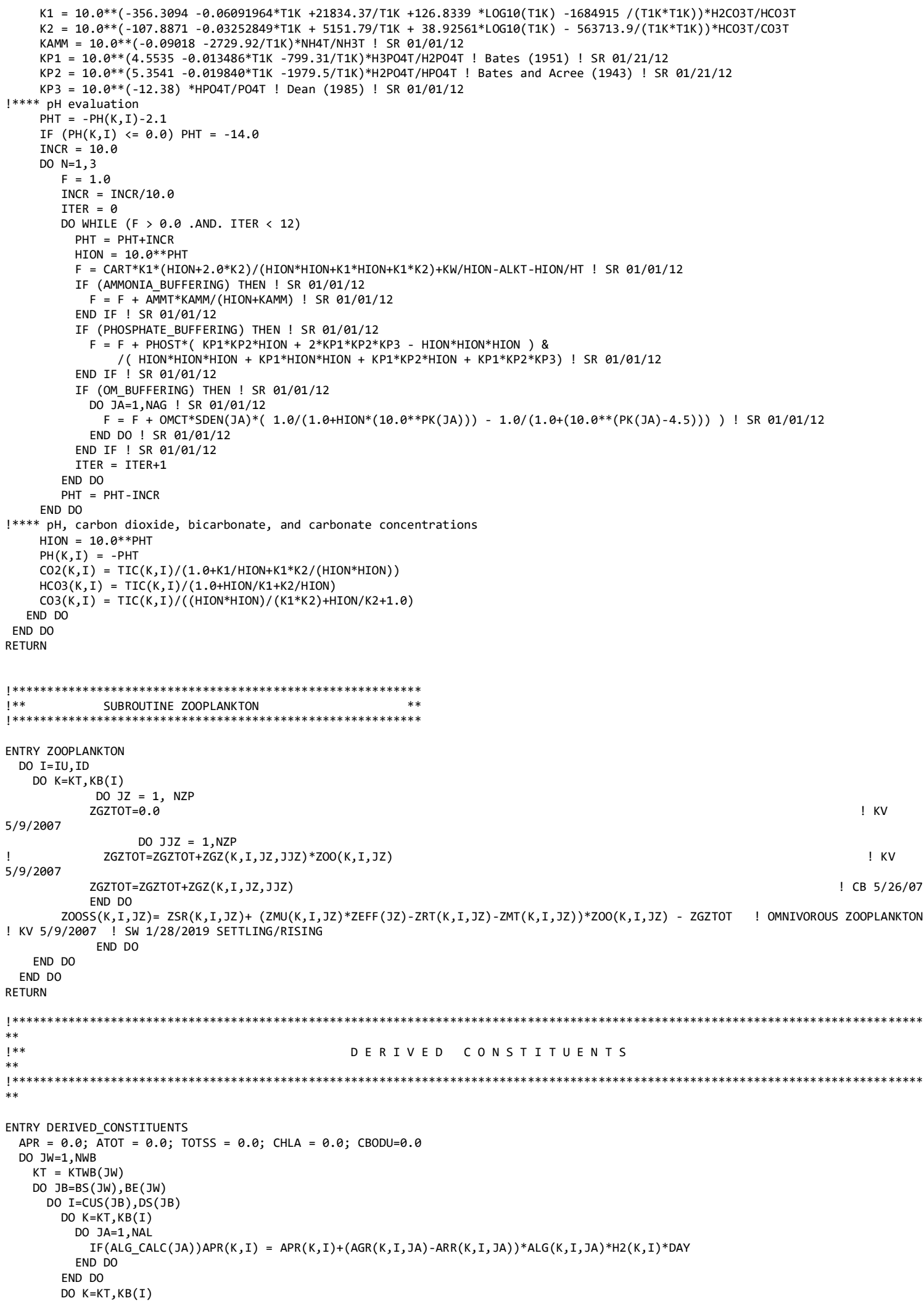




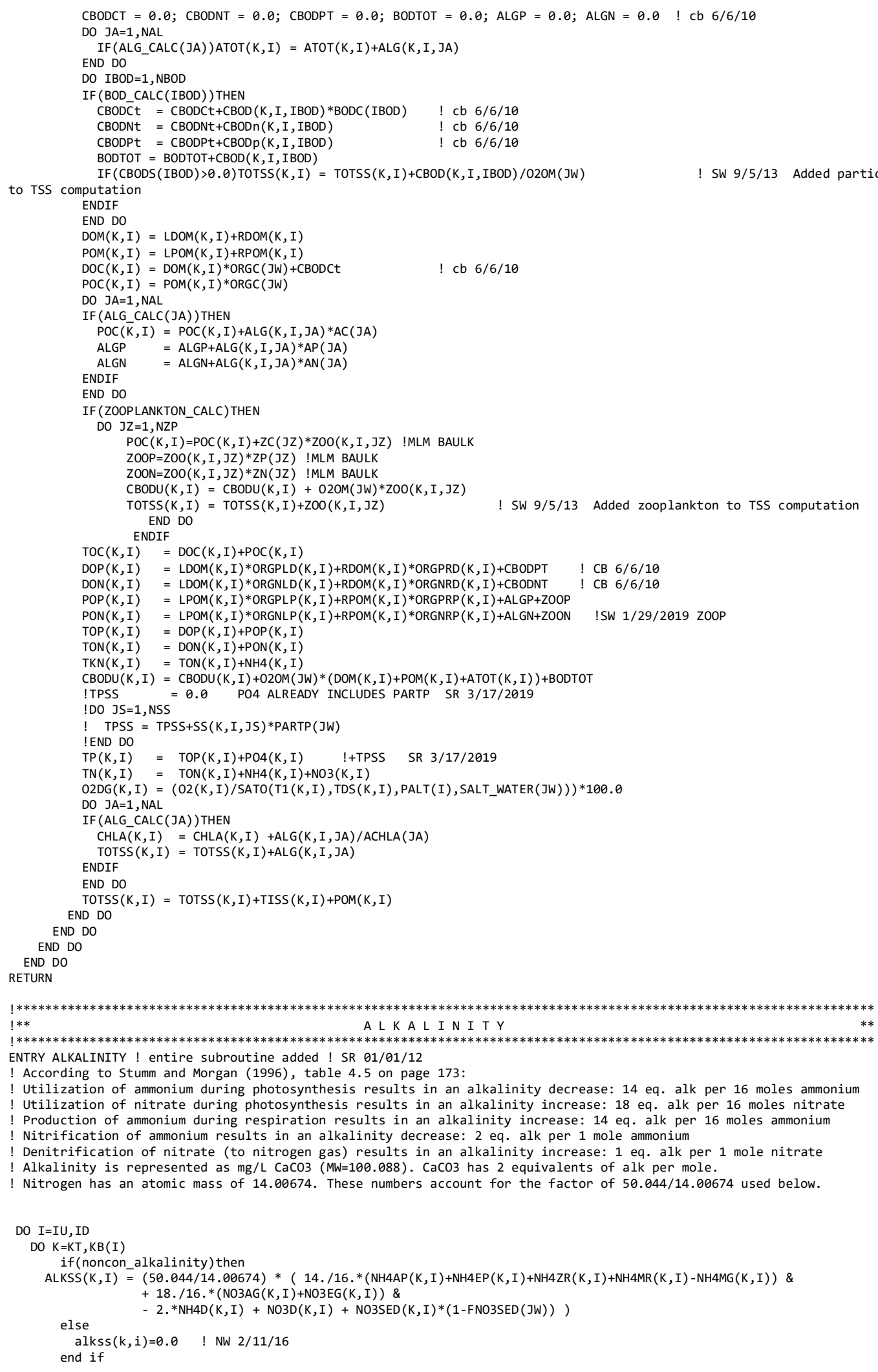


END DO
END DO

RETURN

ENTRY DEALLOCATE KINETICS

DeAllocate (OMTRM, SODTRM, NH4TRM, NO3TRM, DOM, POM, P04BOD, NH4BOD, TICBOD, ATRM, ATRMr, ATRMF, ETRM, etrmR, etrmF, BIBH2)

DEALLOCATE (LAM2M)

DEALlOCATE (ASETTLE, DEN_AVG, DENP, DEN1, DEN2, ALLIM_OLD, DEN, AMP, PHASE, C_COEFF_EXT, RAD, MIND, MAXD, DENSI, DENBI, T_DEC,

C_DENINC, C_DENDEC, DEPTH_LIM, LOSS_FRAC, TWQ, \&

I_C, C_DENINC_1, C_DENINC_2, C_DENDEC_1, C_DENDEC_2, DENP_MINS, DENP_MINB, DENP_MIN, DEN_COR, MIGRATE_GROUP, MIGRATE_MODEL,

TS_DEC, DEPTH LIM ONOFF, ALGAE SETTLING, \&

NMINT, MIGON, MIGOFF, LOLD, DEPTH_CALC_ONOFF, EXP_DEPTH) ! CO 6/12/2019

RETURN

END SUBROUTINE KINETICS 